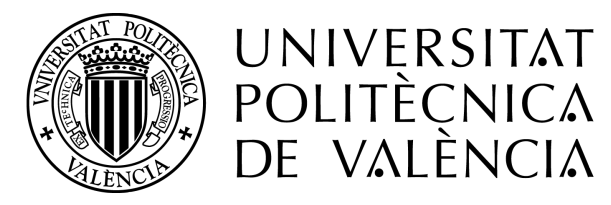

DEPARTAMENTO DE MATEMÁTICA APLICADA

\title{
COMPUTATIONAL METHODS FOR RANDOM DIFFERENTIAL EQUATIONS: THEORY AND APPLICATIONS
}

\section{AUTHOR}

ANA NAVARRO QUILES

ADVISERS

PhD JUAN CARLOS CORTÉS LÓPEZ

PhD MARÍA DOLORES ROSELLÓ FERRAGUD

PhD RAFAEL JACINTO VILLANUEVA MICÓ

València, December 10, 2017 

Don Juan Carlos Cortés López, Catedrático de Universidad de la Universitat Politècnica de València; Doña María Dolores Roselló Ferragud, Titular de Universidad de la Universitat Politècnica de València; y Don Rafael Jacinto Villanueva Micó, Catedrático de Universidad de la Universitat Politècnica de València

\section{CERTIFICAN}

que la presente memoria 'Computational Methods for Random Differential Equations: Theory and Applications' ha sido realizada bajo nuestra dirección por Ana Navarro Quiles y constituye su tesis para optar al grado de Doctora en Ciencias Matemáticas, con mención 'Doctor Internacional'.

Y para que así conste en cumplimiento de la legislación vigente presentamos y apadrinamos ante la Escuela de Doctorado de la Universitat Politècnica de València la referida tesis firmando el presente certificado.

València, noviembre de 2017

Los directores

$\begin{array}{ccc}\text { Juan Carlos } & \text { María Dolores } & \text { Rafael Jacinto } \\ \text { Cortés López } & \text { Roselló Ferragud } & \text { Villanueva Micó }\end{array}$



'I believe that we do not know anything for certain, but everything probably.'

- Christiaan Huygens, Oeuvres Completes. 



\section{Agradecimientos}

De una forma $\mathrm{u}$ otra en esta tesis han contribuido muchas personas, y me gustaría dedicarles unas palabras para agradecerles su apoyo y dedicación.

En primer lugar quiero expresar mi más sincera gratitud a mis directores de tesis, los profesores Juan Carlos, Dolors y Rafa, y también a Vicent, que aunque no haya sido director ha formado parte igualmente de mi formación y de la investigación realizada. Quiero agradecerles la confianza que han depositado en mí para realizar esta tesis y todo el tiempo que me han dedicado. Cada uno de ellos ha aportado su granito (o montaña) de arena. Su guía en todo este recorrido, su amor por las matemáticas que me han transmitido día tras día, su paciencia a la hora de explicarme conceptos totalmente nuevos para mí, todo el tiempo dedicado en cada congreso para mejorar mi inglés. Podría seguir enumerando todo lo que me han aportado estos años, pero no existen palabras ni espacio suficiente. Gracias de corazón, gracias a todos.

Agradecer también a los miembros del Instituto Universitario de Matemática Multidisciplinar por su ayuda cuando la he necesitado. Sobre todo a Cristina, que junto a Rafa, me han enseñado como funciona el mundo de la docencia. También dedicarles unas palabras a los miembros del Departamento de Matemática Aplicada por echarme una mano con la parte administrativa.

Como no, a todas esas personas que me han hecho compañía, ayudándome a desconectar, en el amuerzo durante estos años, y que me han hecho ser la infiltrada en el IUMPA. Gracias también por esos momentos en la que siempre 
será "la casa de Juanmi". Añadir al resto de amigos, que me han acompañado durante estos años, por haber formado parte de mi vida.

A mis padres, sin su apoyo incondicional no habría llegado hasta aquí. Esas conversaciones con mi madre durante la carrera que me animaron a seguir adelante, a no rendirme. Gracias a ella, soy lo que soy, gracias Mamá.

Y finalmente, pero no menos importante, a Bernat, que ha estado en todo momento junto a mí. Aguantando mis locuras y desvelos cuando algo no salía. Gracias por entender lo importante que es y por hacerme reír cuando peor he estado. Contigo todo ha sido más fácil.

Ahora solo queda decir

¡LO CONSEGUIMOS! 
Esta memoria ha sido elaborada en el Instituto Universitario de Matemática Multidisciplinar (IMM) de la Universitat Politècnica de València, durante el periodo de disfrute de un Contrato Pre-Doctoral UPV 2014-Subprograma 1 (Universitat Politècnica de València).

La Ayuda a la movilidad FPI concedida por la Universitat Politècnica de València ha permitido a la autora realizar una estancia de investigación de 3 meses en la University of Manchester, Reino Unido (septiembre-noviembre de 2016) en el Department of Applied Mathematics and Numerical Analysis of School of Mathematics. La bolsa de viaje concedida por la Fundación Ferran Sunyer i Balaguer ha permitido a la autora realizar una estancia de investigación de 3 meses en la Universidad de Sevilla, España (septiembre-noviembre de 2017) en el Departamento de Ecuaciones Diferenciales y Análisis Numérico.

Esta investigación también ha sido financiada parcialmente por el proyecto del Ministerio de Economía y Competitividad: MTM2013-41765-P. 



\section{Resumen}

Desde las contribuciones de Isaac Newton, Gottfried Wilhelm Leibniz, Jacob y Johann Bernoulli en el siglo XVII hasta ahora, las ecuaciones en diferencias y las diferenciales han demostrado su capacidad para modelar satisfactoriamente problemas complejos de gran interés en Ingeniería, Física, Epidemiología, etc. Pero, desde un punto de vista práctico, los parámetros o inputs (condiciones iniciales/frontera, término fuente y/o coeficientes), que aparecen en dichos problemas, son fijados a partir de ciertos datos, los cuales pueden contener un error de medida. Además, pueden existir factores externos que afecten al sistema objeto de estudio, de modo que su complejidad haga que no se conozcan de forma cierta los parámetros de la ecuación que modeliza el problema. Todo ello justifica considerar los parámetros de la ecuación en diferencias o de la ecuación diferencial como variables aleatorias o procesos estocásticos, y no como constantes o funciones deterministas, respectivamente. Bajo esta consideración aparecen las ecuaciones en diferencias y las ecuaciones diferenciales aleatorias. Esta tesis hace un recorrido resolviendo, desde un punto de vista probabilístico, distintos tipos de ecuaciones en diferencias y diferenciales aleatorias, aplicando fundamentalmente el método de Transformación de Variables Aleatorias. Esta técnica es una herramienta útil para la obtención de la función de densidad de probabilidad de un vector aleatorio, que es una transformación de otro vector aleatorio cuya función de densidad de probabilidad es conocida. En definitiva, el objetivo de este trabajo es el cálculo de la primera función de densidad de probabilidad del proceso estocástico solución en diversos problemas basados en ecuaciones en diferencias y diferenciales aleatorias. El interés por determinar la primera función de densidad de probabilidad se justifica porque dicha función determinista caracteriza la información probabilística unidimensional, 
como media, varianza, asimetría, curtosis, etc., de la solución de la ecuación en diferencias o diferencial correspondiente. También permite determinar la probabilidad de que acontezca un determinado suceso de interés que involucre a la solución. Además, en algunos casos, el estudio teórico realizado se completa mostrando su aplicación a problemas de modelización con datos reales, donde se aborda el problema de la estimación de distribuciones estadísticas paramétricas de los inputs en el contexto de las ecuaciones en diferencias y diferenciales aleatorias. 


\section{Resum}

Des de les contribucions de Isaac Newton, Gottfried Wilhelm Leibniz, Jacob i Johann Bernoulli al segle XVII fins a l'actualitat, les equacions en diferències i les diferencials han demostrat la seua capacitat per a modelar satisfactòriament problemes complexos de gran interés en Enginyeria, Física, Epidemiologia, etc. Però, des d'un punt de vista pràctic, els paràmetres o inputs (condicions inicials/frontera, terme font i/o coeficients), que apareixen en aquests problemes, són fixats a partir de certes dades, les quals poden contenir errors de mesura. A més, poden existir factors externs que afecten el sistema objecte d'estudi, de manera que, la seua complexitat faça que no es conega de forma certa els inputs de l'equació que modelitza el problema. Tot açò justifica la necessitat de considerar els paràmetres de l'equació en diferències o de la equació diferencial com a variables aleatòries o processos estocàstics, i no com constants o funcions deterministes. Sota aquesta consideració apareixen les equacions en diferències i les equacions diferencials aleatòries. Aquesta tesi fa un recorregut resolent, des d'un punt de vista probabilístic, diferents tipus d'equacions en diferències i diferencials aleatòries, aplicant fonamentalment el mètode de Transformació de Variables Aleatòries. Aquesta tècnica és una eina útil per a l'obtenció de la funció de densitat de probabilitat d'un vector aleatori, que és una transformació d'un altre vector aleatori i la funció de densitat de probabilitat és del qual és coneguda. En definitiva, l'objectiu d'aquesta tesi és el càlcul de la primera funció de densitat de probabilitat del procés estocàstic solució en diversos problemes basats en equacions en diferències i diferencials. L'interés per determinar la primera funció de densitat es justifica perquè aquesta funció determinista caracteritza la informació probabilística unidimensional, com la mitjana, variància, asimetria, curtosis, etc., de la solució de l'equació en dife- 
rències o l'equació diferencial aleatòria corresponent. També permet determinar la probabilitat que esdevinga un determinat succés d'interés que involucre la solució. A més, en alguns casos, l'estudi teòric realitzat es completa mostrant la seua aplicació a problemes de modelització amb dades reals, on s'aborda el problema de l'estimació de distribucions estadístiques paramètriques dels inputs en el context de les equacions en diferències i diferencials aleatòries. 


\section{Abstract}

Ever since the early contributions by Isaac Newton, Gottfried Wilhelm Leibniz, Jacob and Johann Bernoulli in the XVII century until now, difference and differential equations have uninterruptedly demonstrated their capability to model successfully interesting complex problems in Engineering, Physics, Chemistry, Epidemiology, Economics, etc. But, from a practical standpoint, the application of difference or differential equations requires setting their inputs (coefficients, source term, initial and boundary conditions) using sampled data, thus containing uncertainty stemming from measurement errors. In addition, there are some random external factors which can affect to the system under study. Then, it is more advisable to consider input data as random variables or stochastic processes rather than deterministic constants or functions, respectively. Under this consideration random difference and differential equations appear. This thesis makes a trail by solving, from a probabilistic point of view, different types of random difference and differential equations, applying fundamentally the Random Variable Transformation method. This technique is an useful tool to obtain the probability density function of a random vector that results from mapping another random vector whose probability density function is known. Definitely, the goal of this dissertation is the computation of the first probability density function of the solution stochastic process in different problems, which are based on random difference or differential equations. The interest in determining the first probability density function is justified because this deterministic function characterizes the one-dimensional probabilistic information, as mean, variance, asymmetry, kurtosis, etc. of corresponding solution of a random difference or differential equation. It also allows to determine the probability of a certain event of interest that involves 
the solution. In addition, in some cases, the theoretical study carried out is completed, showing its application to modelling problems with real data, where the problem of parametric statistics distribution estimation is addressed in the context of random difference and differential equations. 


\section{Contents}

List of Figures $\quad$ xxi

List of Tables $\quad$ xxix

List of Publications $\quad$ xxxiii

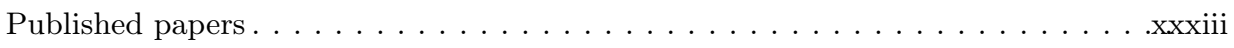

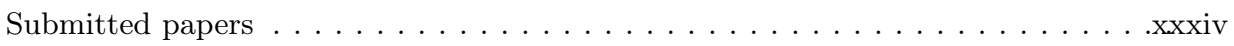

Glossary $\quad$ Xxxv

1 Introduction 1

2 Preliminaries $\quad 7$

2.1 Probability, random variables and stochastic processes . . . . . . . 8

2.2 Random Variable Transformation method . . . . . . . . . . . . . . . . 21

3 Random homogeneous Riccati differential equation $\quad 27$

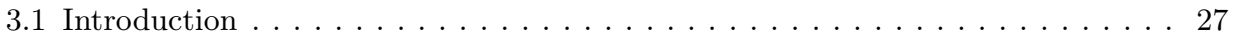




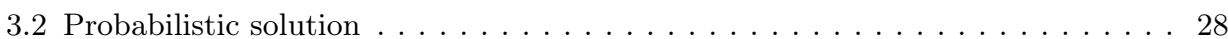

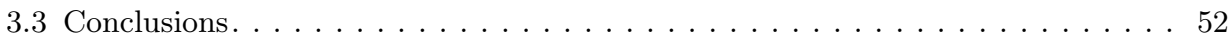

4 Random SIS-type epidemiological models $\quad 53$

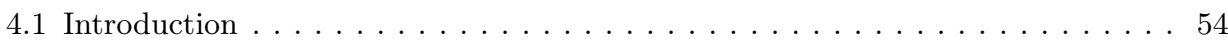

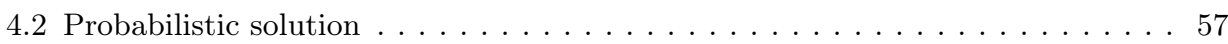

4.3 An illustrative example . . . . . . . . . . . . . . . . . . . 65

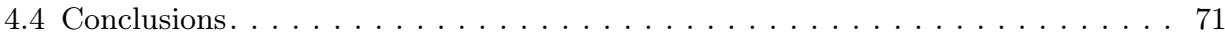

5 Random Bernoulli differential equation $\quad 73$

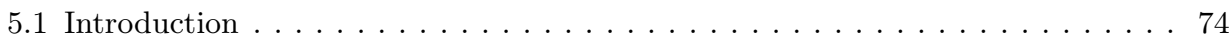

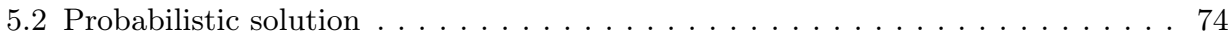

5.3 An application of Bernoulli random differential equation to modelling. . . . . . . 81

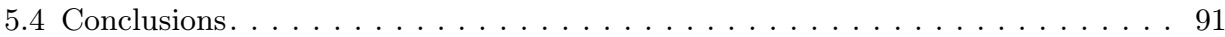

6 Random first-order linear systems of ordinary differential and difference equations $\quad 93$

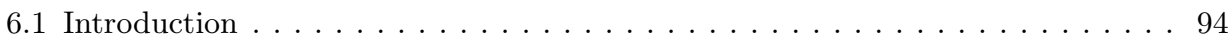

6.2 Random autonomous first-order linear systems of ordinary differential equa-

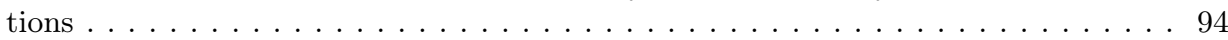

6.3 Random autonomous first-order linear systems of difference equations. . . . . . . 101

6.4 Conclusions. . . . . . . . . . . . . . . . . . . . . . . . . . . . . . . . . . . 109

7 Randomized binary Markov chains 111

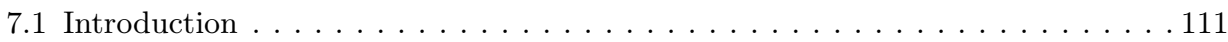

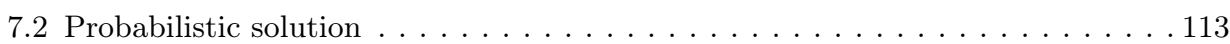

7.3 Relevant probability distributions associated to randomized Markov chains . . . 117

7.4 An application to model the spread of a technology . . . . . . . . . . . . 123

7.5 Conclusions. . . . . . . . . . . . . . . . . . . . . . . . 130

8 Probabilistic solution of a Markov chain to model stroke disease 133

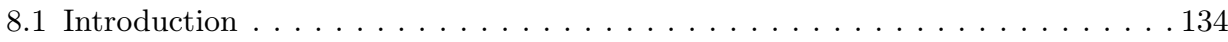




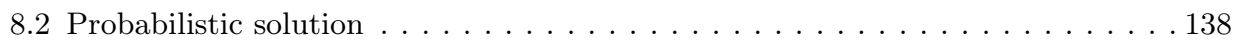

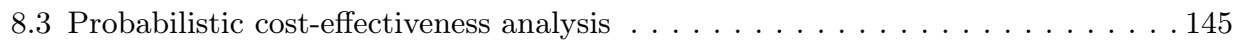

8.4 Simulating stroke disease using real data . . . . . . . . . . . . . . 147

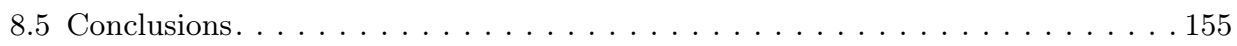

9 Random second-order linear differential equations $\quad 159$

9.1 Introduction . . . . . . . . . . . . . . . . . . . . . . . 159

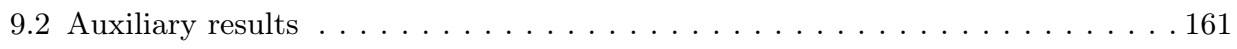

9.3 Computing the approximation of the first probability density function . . . . . 164

9.4 Numerical examples . . . . . . . . . . . . . . . . . . . . . 173

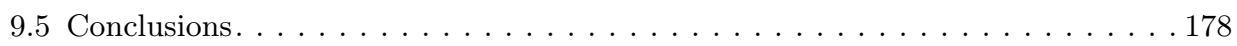

10 Random non-autonomous first-order linear homogeneous differential equations 179

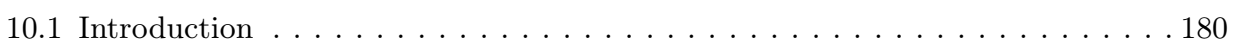

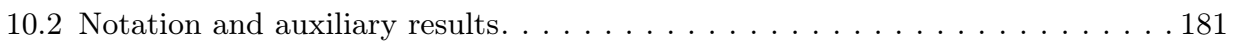

10.3 Computing the 1-PDF of truncated solution stochastic process. . . . . . . . 184

10.4 Numerical examples . . . . . . . . . . . . . . . . . . . . . . . 204

10.5 Conclusions . . . . . . . . . . . . . . . . . . . . . . 214

11 Conclusions $\quad 215$

$\begin{array}{ll}\text { Bibliography } & 219\end{array}$ 



\section{List of Figures}

3.1 Plot of the 1-PDF $f_{1}(x, t)$ given by (3.9) in Example 3.1 at different values of $t \in\{0,0.25,0.5,0.75, \ldots, 2\}$ (corresponding to the solid lines) with $X_{0} \sim \operatorname{Exp}(\lambda=1)$ and $d=-1 . \quad \ldots \quad 34$

3.2 Plot of the expectation (left) and the variance (right) of solution SP in Example 3.1 with $0 \leq t \leq 5 . \ldots \ldots 35$

3.3 Plot of the 1-PDF $f_{1}(x, t)$ given by (3.13) in Example 3.2 at different values of $t \in\{0,0.25,0.5,0.75, \ldots, 2\}$ (corresponding to the solid lines) with $x_{0}=1$ and $D \sim \mathrm{N}(0 ; 1) \ldots \ldots 36$

3.4 Plot of the 1-PDF $f_{1}(x, t)$ in Example 3.3 at different values of $t \in\{0,0.25,0.5,0.75, \ldots, 2\}$ (corresponding to the solid lines) in the case that $\left(X_{0}, D\right)$ has the joint PDF given by (3.16). .

3.5 Plot of the 1-PDF $f_{1}(x, t)$ given by (3.19) in Example 3.4 at different values of $t \in\{0,0.25,0.5,0.75, \ldots, 2\}$ (corresponding to the solid lines) with $c=1 / 2, d=-1$ and $X_{0} \sim \mathrm{N}(0 ; 1)$. .

3.6 Plot of the 1-PDF $f_{1}(x, t)$ given by (3.21) in Example 3.5 at different values of $t \in\{0,0.25,0.5,0.75, \ldots, 2\}$ (corresponding to the solid lines) with $x_{0}=1, c=-1$ and $D \sim \mathrm{Ga}(4 ; 2) \ldots$.

3.7 Plot of the 1-PDF $f_{1}(x, t)$ in Example 3.6 at different values of $t \in\{0.1,0.2,0.3,0.4\}$ with $x_{0}=1, d=1$ and $C \sim \operatorname{Be}(\alpha=$ $2 ; \beta=3)$. 
3.8 Plot of the 1-PDF $f_{1}(x, t)$ in Example 3.7 at different values of $t \in\{0,0.25,0.5,0.75, \ldots, 2\}$ (corresponding to the solid lines) in the case that $c=-1$ and $X_{0}$ and $D$ are correlated RVs according to a bivariate Gaussian distribution with mean vector $\boldsymbol{\mu}$ and variance-covariance matrix $\boldsymbol{\Sigma}$ given by (3.25). . . . . . 44

3.9 Plot of the 1-PDF $f_{1}(x, t)$ in Example 3.8 at different values of $t \in\{0,0.25,0.5,0.75, \ldots, 2\}$ (corresponding to the solid lines) in the case that $d=1$ and $X_{0}$ and $C$ are correlated RVs according to a bivariate Gaussian distribution with mean vector $\boldsymbol{\mu}$ and variance-covariance matrix $\boldsymbol{\Sigma}$ given by (3.25) . . . . . . . .

3.10 Plot of the 1-PDF $f_{1}(x, t)$ in Example 3.9 at different values of $t \in\{0.2,0.5,0.9,1\}$ in the case that $x_{0}=1$ and $(D, C)$ has the joint PDF given by $(3.30) \ldots \ldots \ldots \ldots$

3.11 Plot of the 1-PDF $f_{1}(x, t)$ in Example 3.10 at different values of $t \in\{0,0.5,1,1.5\}$ in the case that $\left(X_{0}, D, C\right)$ has a multivariate Gaussian distribution with mean vector $\boldsymbol{\mu}$ and variancecovariance matrix $\boldsymbol{\Sigma}$ given by (3.34). . . . . . . . . . . . 51

4.1 Flow diagram representation of SIS model $(4.1)-(4.2) . \ldots . \quad 55$

4.2 Plot of $f_{1}(s, t)$ in Example 4.1 during the period 1987-2006 (corresponding to the solid lines). . . . . . . . . . .

4.3 Plot of expectation function (left) and standard deviation function (right) in Example 4.1. . . . . . . . . . . . . . 70

4.4 Expectation (solid line) and confidence intervals (dotted lines) in Example 4.1. . . . . . . . . . . . . . . . . . . . 70

4.5 Plot of the 1-PDF of time $T_{S}$ until a proportion $\rho_{S} \in\{0.45,0.50$, $0.55,0.60,0.65,0.70,0.75\}$ (corresponding to the solid lines) of population remains susceptible in Example 4.1. . . . . . . . .

5.1 Plot of the 1-PDF $f_{1}(x, t)$ given by (5.6) in Example 5.1 at values of $t \in\{0,0.5,1,1.5, \ldots, 5\}$ (corresponding to the solid lines).

5.2 Plot of the 1-PDF $f_{1}(x, t)$ given by (5.9) in Example 5.2 at the values of $t \in\{0,0.5 .1,1.5, \ldots, 6\}$ (corresponding to the solid lines). 82 
5.3 Left: Model fitting to fish weights using least mean squares method. Right: Residuals at the $N=33$ fish weights data. . . 86

5.4 Q-Q plot for the least mean squares residuals. . . . . . . . 87

5.5 Analytic sensibility values of matrix (5.18). Left: $\frac{\partial W}{\partial W_{0}}\left(t_{i} ; \mathbf{Q}\right)$. Right: $\frac{\partial W}{\partial \eta}\left(t_{i} ; \mathbf{Q}\right)$. Center: $\frac{\partial W}{\partial \lambda}\left(t_{i} ; \mathbf{Q}\right) . t_{i}=i, 1 \leq i \leq 33=N \ldots$

5.6 Left: 1-PDF of solution SP to random Bertalanffy model (5.10) given by (5.13) for all time of sample, $t \in\{2, \ldots, 33\}$. Model parameters are assumed to have multivariate Gaussian distribution defined by (5.20), (5.15) and (5.19). Right: Detailed representation of the $1-\mathrm{PDF}$ for times $t \in\{1.1,1.2, \ldots, 2\}$.

5.7 Expectation (solid line) and 99\%-confidence intervals (dotted lines). Points represent fish weigh. . . . . . . . . . . . .

6.1 Top: Phase portrait. Continuous (black) spiral line represents the expectation of solution. 50\% (blue curve) and 90\% (red curve) confidence regions are plotted at time instants $\hat{t} \in\{0,0.5$, $1\}$. Bottom left: PDF of solution at time instant $\hat{t}=0.5$. Bottom right: $\mathrm{PDF}$ of solution at time instant $\hat{t}=1$. On the PDFs plots $50 \%$ and $90 \%$ confidence regions have been highlighted. All these graphical representations correspond to Example 6.1.

6.2 Graphical representation of the stability classification of zeroequilibrium point to random matrix difference equation (6.10) with $m=2$ (random planar system). Each relevant region has a label corresponding to: I (unstable spiral); II (unstable node or source); III (stable spiral); IV (stable node or sink) and V (saddle point). Magenta point has been obtained from matrix $\overline{\mathbf{a}}$ of averaged system (6.16) associated to random system (6.10) with $m=2$ and random inputs given by (6.14)-(6.15). Blue-red confidence regions have been determined from (6.9). . . . . . .

6.3 Top: Phase portrait of random system of difference equations (6.10) with $m=2$ where random input vector $\mathbf{Z}$ has the Gaussian multivariate distribution given in (6.14)-(6.15). Solid line connects the mean and rings represent confidence regions at $50 \%$ and $90 \%$ confidence levels at every value of $n \in\{0,1,2,3\}$. Bottom left and right: PDFs of solution SP at $n=2$ and $n=3$, respectively, together with the corresponding confidence regions. 109 
7.1 Flow diagram to a binary Markov chain. . . . . . . . . . . . 112

7.2 Expectation of postpaid mobile lines (solid line) and 75-95\% confidence intervals (dotted lines). Points represent real data. . 126

7.3 Left: Plot of $f_{1}^{X^{1}}(x ; n)$ given by $(7.3)$ for several cycles and $f_{X_{\infty}^{1}}(x)$ given by (7.6). Right: Plot of $f_{1}^{X^{2}}(x ; n)$ given by $(7.4)$ for several cycles and $f_{X_{\infty}^{2}}(x)$ given by $(7.7) \ldots \ldots 127$

7.4 Plot of $f_{N_{1}}(n)$ given by $(7.12)$ for several values of $\rho_{1}$. . . 128

7.5 1-PDF of the probability of first return $f_{1,1}^{(n)}$ given by (7.16) at $n=1$.

7.6 Left: 1-PDF of the probability of first return $f_{1,1}^{(n)}$ given by (7.16) at cycles $n \in\{2,4, \ldots, 20\}$ (Corresponding to the solid lines). Right: Expectation of $f_{1,1}^{(n)}$ plus/minus standard deviation for $n \geq 2$.

7.7 Top-left: 1-PDF of the probability of first return $f_{2,1}^{(n)}$ at cycles $n \in\{2,3, \ldots, 20\}$. Top-right: Zoom of $1-\mathrm{PDF}$ of the probability of first return $f_{2,1}^{(n)}$ at cycles $n \in\{2,3, \ldots, 9\}$. Bottom: Expectation of $f_{2,1}^{(n)}$ plus/minus standard deviation for $n \geq 2 . \quad$. 130

7.8 PDF of the expected hitting time of state $j$ starting from state $i, m_{i, j}$, given by $(7.18)-(7.21) \ldots \ldots \ldots \ldots 131$

8.1 Flow diagram for Markov model (8.1)-(8.2). $S, R$ and $D$ stand for Susceptible $(S)$, Reliant $(R)$ and Deceased $(D) \ldots \ldots \ldots$

8.2 Plot of the 1-PDFs, $f_{1}(s, n), f_{1}(r, n)$ and $f_{1}(d, n)$, given by (8.10), (8.11) and (8.12) respectively, at values $n \in\{1,2, \ldots, 25\}$ (left: top, center and bottom respectively). For the sake of clarity last times, $n=24$ and $n=25$, have been magnified (right).

8.3 Plot of expectation plus/minus standard deviation functions of susceptible, reliant and deceased subpopulations (top left, top right and bottom, respectively). . . . . . . . . . 150

8.4 Plot of the PDF of time $N_{S}$ until a proportion $\rho_{S} \in\{0.2,0.3$, $0.4,0.5,0.6,0.7,0.8,0.9\}$ of population remains susceptible. . . 151 
8.5 Plot of the PDF of the time $N_{R}$ until a proportion $\rho_{R}=0.006$ of the population remains reliant, using as seed points $s p=1$ (left) and $s p=25$ (right). . . . . . . . . . . . . 152

8.6 Plot of the PDF of time $N_{R}$ until a proportion $\rho_{R}=0.0183$ of population remains reliant, using as seed point $s p=2 \ldots$. . . . 152

8.7 Plot of the PDF of time $N_{D}$ until a proportion $\rho_{D} \in\{0.1,0.2$, $0.3,0.4,0.5,0.6,0.7,0.8,0.9\}$ of population remains dead (left). For the sake of clarity last proportions, $\rho_{D}=0.8$ and $\rho_{D}=0.9$, have been magnified (right). . . . . . . . . . . .

8.8 Plots of expectation of susceptibles, reliants and deceaseds for the two alternatives 1 (the cheapest) and 2 (the most expensive) in three ages: 30 years (first row), 65 years (second row) and 71 years (third row)

8.9 PDFs $C E$ given by (8.19) considering both alternatives in the three ages: 30 years (left), 65 years (center) and 71 years (right). 156

8.10 Plots of expectation plus/minus standard deviation functions of $C E$ for each age considering both alternatives in the three ages: 30 years (left), 65 years (center) and 71 years (right). Red straight line represents the threshold value $30000 € /$ QLY usually taking as reference [78].

9.1 Left: Comparison of approximations, $f_{1}^{N}(x, t)$, of the 1-PDF of solution SP to random IVP (9.1) taking as series order truncation $N \in\{0,1,2\}$ and the corresponding exact 1-PDF $f_{1}(x, t)$ at $t=1$. Right: For ease of comparison, function $f_{1}^{N}(x, 1)$, with $N=2$, has been plotted together with the exact 1-PDF $f_{1}(x, 1) .174$

9.2 Left: Comparison of approximations, $f_{1}^{N}(x, t)$, of the 1-PDF of solution SP to random IVP (9.1) taking as series order truncation $N \in\{0,1,2,3\}$ and the corresponding exact 1-PDF $f_{1}(x, t)$ at $t=1.5$. Right: For ease of comparison, function $f_{1}^{N}(x, 1.5)$, with $N=3$, has been plotted together with the exact 1-PDF $f_{1}(x, 1.5)$. 
9.3 Left: Comparison of approximations, $f_{1}^{N}(x, t)$, of the 1-PDF of solution SP to random IVP (9.1) taking as series order truncation $N \in\{0,1,2,3,4\}$ and the corresponding exact 1-PDF $f_{1}(x, t)$ at $t=2$. Right: For ease of comparison, function $f_{1}^{N}(x, 2)$, with $N=4$, has been plotted together with the exact 1 -PDF $f_{1}(x, 2)$.

9.4 Approximations, $f_{1}^{N}(x, 0.5)$, of the 1-PDF of solution SP to random IVP (9.28) taking as series order truncation $N \in\{0,1,2\}$.

9.5 Left: Approximations, $f_{1}^{N}(x, 1.5)$, of the 1-PDF of solution SP to random IVP (9.28) taking as series order truncation $N \in$ $\{0,1,2,3,4\}$. Right: To facilitate the graphical assessment of approximations, the functions $f_{1}^{N}(x, 1.5)$, with $N \in\{2,3,4\}$, have been magnified.

9.6 Left: Approximations, $f_{1}^{N}(x, 2.5)$, of the 1-PDF of solution SP to random IVP (9.28) taking as series order truncation $N \in$ $\{0,1,2,3,4,5,6\}$. Right: To facilitate the graphical assessment of approximations, functions $f_{1}^{N}(x, 2.5)$, with $N \in\{4,5,6\}$, have been magnified. . . . . . . . . . . . . . . . . . 177

10.1 Left: 1-PDF of the exact solution SP given by (10.29). Center: 1-PDF of the first truncation given by (10.27)-(10.28) with $N=$ 1. Right: 1-PDF of the second truncation given by (10.27)(10.28) with $N=2$.

10.21 -PDF $f_{1}(x, t)$ of the exact solution SP and the two first truncations $f_{1}^{N}(x, t), N \in\{1,2\}$, for different values of $t$ in the context of Example 10.4.1. Top left: $t=0.1$. Top right: $t=1$. Bottom: $t=2$.

10.3 In the context of Example 10.4.1. Left: Exact mean $\left(\mu_{x}\right)$ of solution and its approximations using truncations of order $N \in$ $\{1,2\}\left(\mu_{x}^{1}\right.$ and $\mu_{x}^{2}$, respectively). Right: Exact standard deviation $\left(\sigma_{x}\right)$ of solution and its approximations using truncations of order $N \in\{1,2,3,4\}\left(\sigma_{x}^{i}, i \in\{1,2,3,4\}\right) \ldots \ldots \ldots 208$

10.4 In the context of Example 10.4.2. Top: 1-PDF of the truncations $N=1$ and $N=2$, with $b=1$ Bottom: 1-PDF of the truncations $N=1$ and $N=2$, with $b=0.1 \ldots \ldots \ldots \ldots$ 
10.51 -PDF of the truncations $N \in\{1,2\}$, for $t=0$, in the context of Example 10.4.2. Left: $b=1$. Right: $b=0.1 . \ldots \ldots 211$

10.6 In the context of Example 10.4.2. Top: Approximations of the mean and standard deviation using different orders of truncation $N \in\{1,2,3\}$ with correlation length parameter $b=1$. Bottom: Approximations of the mean and standard deviation using different orders of truncation $N \in\{1,2,3,4,5\}$ with correlation length parameter $b=0.1 \ldots \ldots \ldots \ldots . \ldots \ldots 212$

10.7 First eigenvalues, $\nu_{j}$, of the covariance function (10.32), in the context of Example 10.4.2 . . . . . . . . . . . . . . 213 



\section{List of Tables}

2.1 Some relevant RVs and their mean and variance. . . . . . . . . 14

2.2 Some relevant RVs and their moments with respect to origin. . 15

3.1 List of different cases in which IVP (3.1) is split to conduct the study and identification for the notation used regarding the involved deterministic/random inputs in IVPs (3.2) and (3.1). . 30

4.1 Percentage of non-smokers Spanish men aged over 16 years old during the period 1987-2006. Source [68]. . . . . . . . . 66

4.2 Probabilities associated to the confidence intervals built according to SIS model. . . . . . . . . . . . . . . . . . 69

4.3 Expectation of time $T_{S}$ until a proportion, $\rho_{S}$, of population remains non-smoker for different values $\rho_{S} . \ldots \ldots 71$

5.1 List of the two cases considered to compute the 1-PDF of solution SP to Bernoulli random IVP (5.1) under assumptions (5.2). 75

5.2 Fish weights $w_{i}$ for walleye species in lbs every year $t_{i}, 1 \leq i \leq$ $33=N,[94] . \ldots \ldots \ldots \ldots \ldots \ldots$

5.3 Shapiro-Walk test to check normality of residuals. . . . . . . 86 
7.1 Number of mobile lines per type of contract (postpaid and prepaid) and total of mobile lines during period 2001-2015 in Spain. Source CNMC [26] . . . . . . . . . . . . . . . . . . . . . . 124

7.2 Number of mobile lines in percentage per type of contract (postpaid and prepaid) during period 2001-2015 in Spain. Source CNMC $[26] \ldots \ldots \ldots \ldots \ldots . . \ldots \ldots$

7.3 Expectation and standard deviation of $f_{N_{1}}(n)$ (given by (7.12)) for several values of $\rho_{1} \ldots \ldots \ldots \ldots \ldots \ldots$

7.4 Expectation and standard deviation of the expected hitting time of state $j$ starting from state $i, m_{i, j} \ldots \ldots \ldots \ldots$

8.1 Expectation of time $N_{S}$ until a proportion, $\rho_{S}$, of population remains susceptible for different values $\rho_{S} \ldots \ldots \ldots 151$

9.1 Error measure $e_{N}$ defined by (9.27) for different time instants, $t \in\{1,1.5,2\}$, and series truncation orders, $N \in\{0,1,2,3,4\}$ in Example 9.1. For every $t$, it has been taken $10^{-2}$ as stopping criterion for the tolerance error to $e_{N}$, then values up to the error is for the first time less than $10^{-2}$ have just collected. Otherwise, error is still less than $10^{-2}$ and it has been denoted by means of an hyphen.$- \ldots \ldots \ldots \ldots \ldots$

9.2 Error measure $\hat{e}_{N}$ defined by (9.29) for different time instants, $t \in\{0.5,1.5,2.5\}$, and series truncation orders, $N \in\{0,1,2,3,4$, $5\}$ in Example 9.2. For every $t, 10^{-2}$ has been taken as stopping criterion for the tolerance error to $\hat{e}_{N}$, then values up to error is for the first time less that $10^{-2}$ have just collected. Otherwise, error is still less than $10^{-2}$ and it has been denoted by means of an hyphen -

10.1 Error measure $e_{N}^{\mathrm{PDF}}(t)$ defined by (10.30) for different time instants, $t \in\{0.1,1,2\}$, and truncation orders $N \in\{1,2\}$, in the context of Example 10.4.1. . . . . . . . . . . . . . . 206

10.2 Values of errors $e_{N}^{\mu}$ and $e_{N}^{\sigma}$ for the mean and standard deviation, given by (10.31) using different orders of truncation $N$, in the context of Example 10.4.1. . . . . . . . . . . . . . . . 206 
10.3 Error measure $\hat{e}_{N}(t)$ defined by (10.34) for time instant $t=0$, and truncation orders, $N \in\{2,3\}$ for $b=1$ and $b=0.1$, in the context of Example 10.4.2. . . . . . . . . . . . . . . . . . . 209

10.4 Errors $\hat{e}_{N}^{\mu}$ and $\hat{e}_{N}^{\sigma}$ for the mean and the standard deviation, defined by (10.35) respectively, using different orders of truncations $(N \in\{2,3,4,5\})$ and correlation length $b=0.1$, in the context of Example 10.4.2. . . . . . . . . . . . . . . . . . 211

10.5 Errors $\hat{e}_{N}^{\mu}$ and $\hat{e}_{N}^{\sigma}$ for the mean and the standard deviation, defined by (10.35) respectively, using different orders of truncations $(N \in\{2,3,4,5\})$ and correlation length $b=1$, in the context of Example 10.4.2. . . . . . . . . . . . . . . . . . 213

10.6 First eigenvalues, $\nu_{j}$, of the covariance function (10.32) for $j \in$ $\{1,2, \ldots, 10\}$ for $b=0.1$ and $b=1$ over the time interval $\mathcal{T}=$ $[-0.5,0.5]$, in the context of Example 10.4.2. . . . . . . 213 



\section{List of Publications}

Theoretical results obtained in this dissertation are published or submitted. Each chapter corresponds with the following contributions. Publications are signed in alphabetical order by authors.

\section{Published papers}

Chapter 3: M.-C. Casabán, J.-C. Cortés, A. Navarro-Quiles, J.-V. Romero, M.-D. Roselló, R.-J. Villanueva. Probabilistic solution of the homogeneous Riccati differential equation: A case-study by using linearization and transformation techniques. J. Comput. Appl. Math., 291: 20-35, 2016. (doi: 10.1016/jcam.2014.11.028). Data Base: JCR-Applied Mathematics (2016). Impact factor: 1.357. Position: 63/255 (Q1).

Chapter 4: M.-C. Casabán, J.-C. Cortés, A. Navarro-Quiles, J.-V. Romero, M.-D. Roselló, R.-J. Villanueva. A comprehensive probabilistic solution of random SIS-type epidemiological models using the Random Variable Transformation technique. Comm. Nonl. Sci. Num. Simul., 32: 199-210, 2016. (doi: 10.1016/j.cnsns.2015.08.009). Data Base: JCRApplied Mathematics (2016). Impact factor: 2.784. Position: 9/255 (Q1).

Chapter 5: M.-C. Casabán, J.-C. Cortés, A. Navarro-Quiles, J.-V. Romero, M.-D. Roselló, R.-J. Villanueva. Computing probabilistic solutions of the Bernoulli random differential equation. J. Comput. Appl. 
Math., 309: 396-407, 2017. (doi: 10.1016/j.cam.2016.02.034). Data Base: JCR-Applied Mathematics (2016). Impact factor: 1.357. Position: 63/255 (Q1).

\section{Chapter 6:}

· J.-C. Cortés, A. Navarro-Quiles, J.-V. Romero, M.-D. Roselló. Probabilistic solution of random autonomous first-order linear systems of ordinary differential equations. Rom. Rep. Phys., 68(4): 1397-1406, 2016. Data Base: JCR-Physics, Multidisciplinary (2016). Impact factor: 1.467. Position: 34/79 (Q2).

· J.-C. Cortés, A. Navarro-Quiles, J.-V. Romero, M.-D. Roselló. Full solution of random autonomous first-order linear systems of difference equations. Application to construct random phase portrait for planar systems. Appl. Math. Lett., 68: 150-156, 2017. (doi: 10.1016/j.aml.2016.12.015). Data Base: JCR-Applied Mathematics (2016). Impact factor: 2.233. Position: 17/255 (Q1).

Chapter 8: J.-C. Cortés, A. Navarro-Quiles, J.-V. Romero, M.-D. Roselló. Randomizing the parameters of a Markov chain to model the stroke disease: A technical generalization of established computational methodologies towards improving real applications. J. Comput. Appl. Math., 324: 225-240, 2017. (doi: 10.1016/j.jcam.2017.04.040 ). Data Base: JCR-Applied Mathematics (2016). Impact factor: 1.357. Position: 63/255 (Q1).

\section{Submitted papers}

Chapter 7: J.-C. Cortés, A. Navarro-Quiles, J.-V. Romero, M.-D. Roselló. Some results about randomized binary Markov chains: Theory, computing and applications.

Chapter 9: J.-C. Cortés, A. Navarro-Quiles, J.-V. Romero, M.-D. Roselló. Solving second-order linear differential equations with random analytic coefficients about regular points: A full probabilistic solution by the first probability density function.

Chapter 10: J.-C. Cortés, A. Navarro-Quiles, J.-V. Romero, M.-D. Roselló. Computing the probability density function of non-autonomous first-order linear homogeneous differential equations with uncertainty. 


\section{Glossary}

RV random variable

2-RV second order random variable

SP stochastic process

2-SP second order stochastic process

DF distribution function

PDF probability density function

PF probability function

1-PDF first probability density function

$n$-PDF $n$-dimensional probability density function

IVP initial value problem

RVT Random Variable Transformation

$\mathcal{D}(\mathbf{X}) \quad$ domain of random vector $\mathbf{X}$ 
$\Omega$

$\mathcal{A}$

$\mathbb{P}$

a.s

w.p.

w.p. 1

i.i.d.

m.s.

$\mathbb{E}[X]=\mu_{X}$

$\mathbb{V}[X]=\sigma_{X}^{2}$

$\sigma_{X}$

$L_{2}$

$\top$

$\mathcal{T}$

$A \sim \operatorname{Be}(\alpha ; \beta)$

$A \sim \mathrm{Ga}(r ; \alpha)$

$A \sim \mathrm{N}(\mu ; \sigma)$

$A \sim \operatorname{Un}([a, b])$

$A \sim \operatorname{Exp}(\lambda)$

$\|X\|_{2}$ sample space

subsets of $\Omega$ called events

probability

almost surely

with probability

with probability one

identical independently distributed

mean square

expectation or mean or average of random variable $X$

variance of random variable $X$

standard deviation of random variable $X$

set of second order scalar random variables

superscript, it denotes the transpose for vectors/matrices

time-space of an arbiratry continuous stochastic process

$A$ has a Beta distribution of parameters $\alpha, \beta>0$

$A$ has a Gamma distribution of parameters $r, \alpha>0$

$A$ has a Gaussian distribution of parameters $\mu \in \mathbb{R}, \sigma>0$

$A$ has a Uniform distribution on the interval $[a, b]$

$A$ has an Exponential distribution of parameter $\lambda>0$

2-norm of random variable $X$ 
set of nonnegative integers

$\mathbb{R}$

set of real numbers

$\mathbb{R}^{n}$

set of vectors of dimension $n$ with real coefficients

$\mathbb{R}^{n \times m}$

set of matrices of size $n \times m$ with real coefficients

$\frac{\mathrm{d}^{n}}{\mathrm{~d} t^{n}}$

ordinary derivative of order $n$ with respect to $t$

$\dot{\mathbf{X}}(t)$

first mean square derivative of $\mathbf{X}(t)$

$\ddot{\mathbf{X}}(t)$

second mean square derivative of $\mathbf{X}(t)$,

$\operatorname{Cov}[X, Y]$

covariance of random variables $X, Y$

$\delta(\cdot)$

Dirac delta function

$\mathrm{L}^{2}\left(\Omega, \mathrm{L}^{2}(\mathcal{T}, \mathrm{H})\right) \quad$ Hilbert space of mean square integrable SPs,

that are valued on a Hilbert space $\left(H,\langle\cdot, \cdot\rangle_{\mathrm{H}}\right)$.

$\mathrm{L}^{2}\left(\Omega, \mathrm{L}^{2}(\mathcal{T})\right) \quad$ Hilbert space of mean square integrable SPs, that are valued on $\mathbb{R}$.

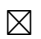

end of the proof of a mathematical result 



\section{Chapter 1}

\section{Introduction}

Difference and differential equations have demonstrated to be important mathematical tools to model different phenomena in many areas. There is virtually no applied scientific area where these equations had not been used to deal with relevant problems. Numerous examples can be found in Engineering, Physics, Chemistry, Epidemiology, Economics, etc. From a practical standpoint, the application of difference or differential equations requires setting their input (coefficients, source term, initial and boundary conditions) using sample data, thus containing uncertainty stemming from measurement errors. Depending on the quality of these measurements, the results obtained from the model can be satisfactory or not. In addition, there are some external sources with a random nature that can affect the system, such as the environmental and genetic factors in Epidemiology, for example. Then, it is more advisable to consider the input data as random magnitudes rather than deterministic ones. If what is to be measured is a magnitude functionally independent of other values, it would be better to consider it as a random variable rather than a deterministic constant. When dependency exists with respect to other magnitudes, such as time, space, etc., it would be more advisable to interpret it, not as a function but as a stochastic process. Under this approach, two main classes of difference or differential equations, which consider into their formulation uncertainty, have been proposed to formulate continuous and dis- 
crete models, namely stochastic difference or differential equations and random difference or differential equations.

The uncertainty or noise considered in stochastic differential equations is considered by special classes of stochastic processes like Markovian processes, or, being more specific the white noise process (the formal derivative of the Wiener process, also called Brownian motion). The white noise restricts itself the uncertainty to a Gaussian process with irregular sample behaviour since the trajectories of the Wiener process are nowhere differentiable [71]. The Brownian motion belongs to an important class of stochastic processes termed semimartingales. The resulting stochastic differential equation is handled using a special calculus for semimartingales, usually referred to as Itô stochastic calculus, whose cornerstone result is the Itô Lemma [2, 71]. The Brownian motion is characterized by Gaussian, stationary and independent increments. From a deterministic point of view, Itô calculus considers perturbations in the parameters via the white noise. This implicitly entails that Gaussian uncertainty is assumed via the perturbation of model parameters. Apart from Wiener process, some authors have also considered other types of stochastic processes, like the coloured noise $[22,23]$. Additionally, stochastic differential equations can be considered as a continuous version of autoregressive models which are widely applied in statistics [89]. But, if it is possible stochastic differential equations are solved using Itô calculus, when not numerical techniques can be developed [57]. Stochastic differential equations have demonstrated to be powerful mathematical representations to model many problems, for example in Finance, Engineering, Biosciences, etc., [14, 36, 40, 93], but this approach does not cover all the probabilistic casuistries.

A complementary approach to introduce uncertainty in differential equations, and also in difference equations, is to allow the direct assignment of any probability distribution to input parameters, which is referred to as the randomization process of deterministic or classical difference or differential equations. Random difference or differential equations constitute a natural generalization of their deterministic counterpart. Random effects are directly manifested in parameters, which are considered random variables or stochastic processes, i.e., parameters are assumed to have regular sample behaviour described by standard probabilistic distributions. The main advantage of random differential equations with respect to their stochastic counterpart is that for the former a wider range of probability distributions for input parameters are allowed, such as, Beta, Gaussian, Exponential as well as other ad hoc distributions, like those that can be built using copulas [70]. A good introduction to random differential equations can be found in [85]. The standard study of random difference or 
differential equations is based upon the so-called mean square calculus. Under this approach, apart from solving the random difference or differential equation, the main objective is to compute the mean and variance of the solution stochastic process of a random difference or differential equation, while this dissertation deals with the computation of the probability density function of the solution stochastic process, which is a more desirable goal. In addition, a number of numerical and analytical methods, which extend their deterministic counterpart, have been proposed to deal with random difference or differential equations including numerical schemes, spectral methods and Fröbenius series, for instance $[13,29,41]$.

A common approach, in order to approximate the solutions of stochastic difference or differential equations and random difference or differential equations, is Monte Carlo sampling [59]. Although widely used due to its easy implementation, the main drawback of Monte Carlo is its slow convergence rate, since the error is $\mathcal{O}(1 / \sqrt{M})$, where $M$ is the number of simulations. In addition, Monte Carlo only provides numerical approximation of the solution stochastic process, in spite of an exact representation could exist.

In this thesis, autonomous and non-autonomous random differential equations and systems of random difference or differential equations will be solved. As main difference with respect to the deterministic framework, solving a random difference or differential equation means not only to compute, exact or approximately, its solution stochastic process, say $X(t)$, and to study dependence of solution with respect to initial conditions and/or parameters, but to determine the main statistical functions of $X(t)$, such as its mean and variance functions, are also important goals to be achieved. Although this information is valuable, and most contributions focus on the computation of the solution stochastic process and its mean and variance/standard deviation functions, a more ambitious target includes the determination of the first probability density function, $f_{1}(x, t)$, in order to have a full probabilistic description of the solution stochastic process in each instant time. Moreover, from the first probability density function one can calculate all the one-dimensional statistical moments, in particular, from it the mean and the variance, and therefore, confidence intervals, can be straightforwardly determined, as well as, skewness, kurtosis and other higher one-dimensional statistical moments. This fact constitutes crucial information from a practical standpoint because it permits to provide more realistic answers than its deterministic counterpart, where predictions are just punctual values. In addition, the first probability density function also enables to compute the probability that the solution lies within a certain set of interest. 
Even though this thesis focusses on the computation of the first probability density function of the solution, it is worth to underline that higher probability density functions are also useful for giving further statistical characteristics. That is, in order to have more probabilistic information, one can calculate the $n$-dimensional probability density function of the solution stochastic process, but it normally involves complex computations. For example, the second probability density function, $f_{2}\left(x_{1}, t_{1} ; x_{2}, t_{2}\right)$, gives a full probabilistic description of the solution stochastic process in each pair of times, $t_{1}$ and $t_{2}$. Particularly, from the second probability density function the correlation function can be calculated. This function provides a measure of linear statistical interdependence between the random variables coming from evaluating the stochastic process in two different time instants. Furthermore, the correlation function allows to compute the covariance function, once the mean function has also been calculated from the first probability density function.

As it was pointed out previously, Itô type stochastic differential equations are an alternative to random differential equations in order to model uncertainty. Taking into account our principal objective, namely the computation of the first probability density function of the solution stochastic process, under the so called Itô approach it is also possible to determine the probability density function solving the corresponding forward Fokker-Plank-Kolmogorov equation. This equation is a deterministic partial differential equation satisfied by the transition probability density function of a diffusion stochastic process, [39, 42]. The key point that allows to use this result is that the solution of Itô type stochastic differential equation is a Markovian diffusion process, [85]. But this fact does not have to be fulfilled under the approach of this dissertation.

The main objective of this dissertation relies on the application of the Random Variable Transformation technique. This method, that will be introduced in the next chapter, is a powerful tool to compute the probability density function of a random vector, which results from mapping another random vector, whose probability density function is known [20]. In [52] a generalization of this technique is stated. This method has been successfully applied to different type of problems: random partial differential equations [49, 51], random integraldifferential equations [50], random difference equations [17], and this technique can be also applied to obtain numerical approximations [37].

The introduction is concluded by summarizing the contents of this dissertation.

- Chapter 2 is concerned with the fundamental principles and results in probability, random variables and stochastic processes that will be required throughout theses pages. In addition, in Chapter 2 a brief intro- 
duction about Random Variable Transformation method will be provided, for the sake of completeness.

- In Chapter 3, the first probability density function of the solution stochastic process to the homogeneous Riccati random differential equation is determined. The study is split in all possible cases regarding the deterministic/random nature of the involved input parameters. An illustrative example is provided for each one of the considered cases.

- Chapter 4 provides a complete probabilistic description of SIS-type epidemiological models. In particular, the distributions to describe the time until a given proportion of the population remains susceptible and infected is also determined and a probabilistic description of the so-called basic reproductive number is included. The theoretical results are applied in order to study the spread of smoking in Spain by using real data.

- In Chapter 5, the first probability density function of the solution stochastic process to the Bernoulli random differential equation is determined. The analysis is split into two cases for which an illustrative example is provided. Finally, a fish weight growth model is considered to illustrate the usefulness of the theoretical results previously established using real data.

- In Chapter 6, the first probability density function of the solution stochastic processes is computed to

- Random autonomous first-order linear systems of ordinary differential equations. In this part the theoretical results are illustrated for planar systems and a probabilistic interpretation of phase portrait is given.

- Random autonomous first-order linear systems of difference equations. In this case the computation of the first probability function is applied to extend the classical stability classification of the zeroequilibrium point.

In both problems, some numerical examples are provided.

- Chapter 7 is addressed to give a generalization of the classical Markov methodology. This generalization allows the treatment of the entries of the transition matrix and initial condition as random variables instead of deterministic values lying in the interval $[0,1]$. Important quantities related to randomized Markov chains (steady state, hitting times, etc.) 
are determined. The theoretical results are illustrated by modelling the diffusion of a technology using real data.

- Chapter 8 is aimed to give a technical generalization of classical Markov methodology in order to improve modelling of the stroke disease. As in Chapter 4, the probability density function of the time instants until a certain proportion of the total population remains susceptible, reliant and deceased are also computed. The study is completed showing the usefulness of out computational approach to determine, from a probabilistic point of view, key quantities in medical decision making, such as the cost-effectiveness ratio.

- In Chapter 9, the first probability density function of the approximate solution stochastic process to second-order linear differential equations with random analytic coefficients about regular point is computed. And then, the study of the convergence to the exact first probability density function is done. The validity of the proposed method and the convergence is shown through several illustrative examples.

- In Chapter 10, the first-order random linear differential equation, where the diffusion coefficient is an stochastic process, is considered. On the one hand, in order to obtain the first probability density function this coefficient is represented via Karhunen-Loève expansion. On the other hand, to apply Random Variable Transformation method, the truncated series is considered. Therefore, the first probability density function of the approximate solution stochastic process will be obtained. Then, the uniform convergence of the sequence of first probability density functions is established applying the classical Cauchy condition. This theoretical result is validated throughout some numerical examples.

- Finally, in Chapter 11 the main conclusions of this thesis are summed up.

For the sake of clarity, from Chapter 3 to Chapter 10 the same structure is followed: a brief introduction; the probabilistic solution, that is, the computation of the first probability density function and the main statistical functions of the solution stochastic process of the corresponding problem, some numerical examples and/or a specific mathematical model, and some conclusions of the chapter. 


\section{Chapter 2}

\section{Preliminaries}

In this chapter some previous results concerned with the principles in probability, random variables and stochastic process, which are fundamental in this dissertation, as well as the Random Variable Transformation method with some specializations will be stated.

The aim of this chapter is twofold. On the one hand, Section 2.1 is addressed to give the fundamental principles and results in probability, Random Variables $(\mathrm{RVs})$ and Stochastic Processes (SPs). Notice that Section 2.1 is based on the preliminaries of thesis [79] given that the mathematical tools explained in this section are common in both dissertations. On the other hand, the main results that will be required in next chapters, are summarized in Section 2.2. That is, in Section 2.2 Random Variable Transformation (RVT) method, with specialization of this technique, will be stated. Since the reader is assumed to be familiar with the basic concepts in these subjects, the chapter is written in a direct way in order to provide a brief introduction and a summary of those results useful in the subsequent development. The reader is encouraged to check classical references like [85], [72], [60], [95], [33], for further details. 


\subsection{Probability, random variables and stochastic processes}

\subsubsection{Probability space}

The principal concept of probability theory is the probability space, which can be represented mathematically as an ordered triplet $(\Omega, \mathcal{A}, \mathbb{P})$, consisting of the sample space, $\Omega$, a $\sigma$-algebra $\mathcal{A}$ of subsets of $\Omega$, called events, and a real-valued set function $\mathbb{P}$, defined on $\mathcal{A}$, called a probability. $\Omega$ can be any arbitrary set, while $\mathcal{A}$ and $\mathbb{P}$ must satisfy the following properties. First, for $\mathcal{A}$

i) $\varnothing \in \mathcal{A}$.

ii) If $A \in \mathcal{A}$, then $A^{C} \in \mathcal{A}$ (where $A^{C}$ denotes the complement of $A$ in $\Omega$ ).

iii) If $A_{1}, A_{2}, \ldots \in \mathcal{A}$, then $A=\bigcup_{i=1}^{\infty} A_{i} \in \mathcal{A}$.

With respect to $\mathbb{P}$

iv) If $A \in \mathcal{A}$, then $\mathbb{P}[A] \geq 0$.

v) If $A_{1}, A_{2}, \ldots \in \mathcal{A}$ is a family of disjoint sets, i.e., $A_{i} \bigcap A_{j}=\varnothing$ for $i \neq j$, then

$$
\mathbb{P}\left[\bigcup_{i=1}^{\infty} A_{i}\right]=\sum_{i=1}^{\infty} \mathbb{P}\left[A_{i}\right]
$$

vi) $\mathbb{P}[\Omega]=1$.

Properties i)-iii) define $\mathcal{A}$ as a $\sigma$-algebra of subsets of $\Omega$, while properties iv)-v) determine that $\mathbb{P}$ is a measure and, vi) defines $\mathbb{P}$ as a probability (measure).

If $A \in \mathcal{A}, \mathbb{P}[A]$, the probability of $A$, can be interpreted as a measure of the likelihood of occurrence of the event $A$ in an experiment whose possible outcomes are the elements of $A$. If $\mathbb{P}[A]=0, A$ is virtually impossible, and $A$ is called a $\mathbb{P}$-null set. If $\mathbb{P}[A]=1, A$ is a certainty event and it is said that $A$ occurs with probability 1 (w.p. 1) or almost surely (a.s.). 


\subsubsection{Random variables}

Now let $\left(\Omega_{1}, \mathcal{A}_{1}\right)$ and $\left(\Omega_{2}, \mathcal{A}_{2}\right)$ be measurable spaces, that is, $\mathcal{A}_{i}$ is a $\sigma$-algebra of subsets of $\Omega_{i}, i=1,2$. If $X$ is a mapping of $\Omega_{1}$ into $\Omega_{2}$, denoted by $X: \Omega_{1} \rightarrow \Omega_{2}$, then $X$ is measurable if for each $A \in \mathcal{A}_{2}$,

$$
\{\omega: X(\omega) \in A\}=X^{-1}(A) \in \mathcal{A}_{1} \text {. }
$$

The collection $\mathcal{A}(X)=\left\{X^{-1}(A): A \in \mathcal{A}_{2}\right\}$ is a $\sigma$-algebra in $\Omega_{1}$, called the $\sigma$-algebra generated by $X$, and it is the smallest $\sigma$-algebra such that $X$ is measurable. The cases where $\Omega_{2}=\mathbb{R}$ (R denotes the set of all real numbers); $\Omega_{2}=\mathbb{R}^{n}$ ( $\mathbb{R}^{n}$ denotes the set of $n$-dimensional vectors with real components); $\Omega_{2}=\mathbb{R}^{n \times m}\left(\mathbb{R}^{n \times m}\right.$ denotes the set of $n \times m$ matrices with real entries $)$ are of particular interest. In these cases $\mathcal{A}_{2}$ is usually taken to be the $\sigma$-algebra of Borel sets of the appropriate dimensions: the $\sigma$-algebra generated by the set of intervals (rectangles, etc.) and denoted by $\mathcal{B}, \mathcal{B}^{n}$ or $\mathcal{B}^{n \times m}$, etc. respectively. Measurable functions $X: \Omega_{1} \rightarrow \Omega_{2}$ are called RVs, random vectors, and random matrices, respectively, in each of these cases. $X$ is a random vector (resp. matrix) if and only if each component $X_{i}$ (resp. $X_{i j}$ ) is a RV. Throughout this thesis a random vector and a random matrix will be denoted by $\mathbf{X}$. A function $X: \Omega_{1} \rightarrow \mathbb{R}$ is a real $\mathrm{RV}$ if, and only if,

$$
\left.\left.X^{-1}(]-\infty, a\right]\right)=\{\omega: X(\omega) \leq a\} \in \mathcal{A}_{1}, \quad \forall a \in \mathbb{R}
$$

Notice that, throughout this dissertation the parameter $\omega$ will be omitted when no confusion seems possible and capital letters will be used when dealing with $\mathrm{RVs}$, random vectors, or random matrices.

If the measurable space $(\Omega, \mathcal{A})$ represents an experiment, a $\mathrm{RV} X$ defined on $(\Omega, \mathcal{A})$ can be considered as an abstraction of information from the possible outcomes. This abstraction can be very coarse as in the case of an indicator function or a constant function. However, the measurably requirement means that only those subsets of $\Omega$ that are events can be distinguished by different values of $X$. This restriction indicates how discerning can $X$ be, in the sense one that the information made available by $X$ about the experiment cannot exceed that provided by the full $\sigma$-algebra $\mathcal{A}$, which describes all possible outcomes of the experiment.

Usual algebraic operations of the analysis are, in general, preserved for the case that RVs are managed. For example, sums, differences, products, and 
when they exist, quotients, extrema, and limits of RVs are also RVs. This situation can be exploited to determine probabilities of compound events from the probabilities of simpler constituent elements.

Given a random experiment with its associated RV $X$ and given a real number $x$, let us consider the probability of the event $\{x: X(\omega) \leq x\}$ or simply $\mathbb{P}[X \leq x]$. This probability is a number, clearly dependent upon $x$. Function

$$
F_{X}(x)=\mathbb{P}[X \leq x]
$$

is defined as the distribution function (DF) of $X$ and always exists. By definition it is a non-negative, continuous to the right and non-decreasing function of real variable $x$. Moreover, it satisfies

$$
F_{X}(-\infty)=0, \quad F_{X}(+\infty)=1 .
$$

A RV $X$ is called a continuous RV if its associated DF is continuous and differentiable almost everywhere. It is a discrete RV when the DF assumes the form of a staircase with a finite or countable finite jumps. For a continuous $\mathrm{RV} X$, the derivative

$$
f_{X}(x)=\frac{\mathrm{d} F_{X}(x)}{\mathrm{d} x}
$$

exists and it is called probability density function (PDF) of RV $X$. This function satisfies the following properties

$$
f_{X}(x) \geq 0, \quad \int_{a}^{b} f_{X}(x) \mathrm{d} x=F_{X}(b)-F_{X}(a), \quad \int_{\mathcal{D}(X)} f_{X}(x) \mathrm{d} x=1,
$$

where $\mathcal{D}(X)$ denotes the domain of $\mathrm{RV} X$. Notice that throughout this thesis if the domain is unknown or cumbersome, the whole real space $((-\infty, \infty))$ will be used. If $X$ is a RV that only takes a finite number of values, say, $x_{1}, x_{2}, \ldots, x_{N}$, its PDF is usually called probability function or mass function and it is defined by

$$
f_{X}(x)=\sum_{j=1}^{N} p_{j} \delta\left(x-x_{j}\right)
$$


where $\delta(x)$ is the Dirac delta function and

$$
p_{j}=\mathbb{P}\left[X=x_{j}\right], \quad j=1,2, \ldots, N
$$

This definition of $f_{X}(x)$ is consistent in sense that it has all properties indicated in expression (2.1), and the DF is recoverable from equation (2.2) by integration.

In order to introduce later the concept of independent RVs, first it is necessary to introduce the concept of DF and PDF for two RVs (for the extension to more than two RVs, see for instance, reference [85, p.13]). Given two RVs $X$ and $Y$, then, they induce a probability distribution in the Euclidean plane $\mathbb{R}^{2}$, which is described by the joint DF

$$
F_{X Y}(x, y)=\mathbb{P}[\{\omega \in \Omega: X(\omega) \leq x, Y(\omega) \leq y\}]
$$

Function $F_{X Y}(x, y)$ is non-negative, non-decreasing, and continuous at the right with respect to each of real variables $x$ and $y$. Moreover, it satisfies the following properties

$$
\begin{gathered}
F_{X Y}(-\infty,-\infty)=F_{X Y}(-\infty, y)=F_{X Y}(x,-\infty)=0 \\
F_{X Y}(+\infty,+\infty)=1, \quad F_{X Y}(x,+\infty)=F_{X}(x), \quad F_{X Y}(+\infty, y)=F_{Y}(y),
\end{gathered}
$$

where $F_{X}(x)$ and $F_{Y}(y)$ denote the individual DF of RVs $X$ and $Y$, respectively, but in the context of several RVs, each (individual) DF is commonly referred to as the marginal distribution function. If the second-order partial derivative

$$
\frac{\partial^{2} F_{X Y}(x, y)}{\partial x \partial y}
$$

exists, it is denoted by

$$
f_{X Y}(x, y)=\frac{\partial^{2} F_{X Y}(x, y)}{\partial x \partial y}
$$


and it is called joint PDF of $X$ and $Y$. This function satisfies similar properties that previously enunciated for an individual PDF in (2.1), that is, it is nonnegative and

$$
\begin{gathered}
\int_{\mathcal{D}(Y)} \int_{\mathcal{D}(X)} f_{X Y}(x, y) \mathrm{d} x \mathrm{~d} y=1, \quad \int_{-\infty}^{x} \int_{-\infty}^{y} f_{X Y}(u, v) \mathrm{d} u \mathrm{~d} v=F_{X Y}(x, y), \\
\int_{\mathcal{D}(Y)} f_{X Y}(x, y) \mathrm{d} y=f_{X}(x), \quad \int_{\mathcal{D}(X)} f_{X Y}(x, y) \mathrm{d} x=f_{Y}(y) .
\end{gathered}
$$

Within this framework, PDFs $f_{X}(x)$ and $f_{Y}(y)$ are called marginal density functions of $\operatorname{RVs} X$ and $Y$, respectively.

Two RVs $X$ and $Y$ are said to be independent if their joint PDF $f_{X Y}(x, y)$ can be factorized as the product of each individual $\operatorname{PDF} f_{X}(x)$ and $f_{Y}(y)$, that is,

$$
f_{X Y}(x, y)=f_{X}(x) f_{Y}(y)
$$

The extension of previous definition to case of more than two RVs is straightforward. A family of $n \mathrm{RVs}, X_{1}, X_{2}, \ldots, X_{n}$, are mutually independent if, and only if,

$$
f_{X_{1}, X_{2}, \ldots, X_{n}}\left(x_{1}, x_{2}, \ldots, x_{n}\right)=f_{X_{1}}\left(x_{1}\right) f_{X_{2}}\left(x_{2}\right) \cdots f_{X_{n}}\left(x_{n}\right)
$$

An important result concerning independence is the following.

Proposition 2.1 ([44]) If $X$ and $Y$ are independent $R V s$, then for any (measurable) functions $f$ and $g, R V s f(X)$ and $g(Y)$ are also independent.

In practice a RV tries to model the uncertainty of a phenomenon. Some of the most useful information concerning a RV is revealed by deterministic quantities called moments that capture its statistical variability. Given a $\mathrm{RV} X$, the $n$-th moment with respect to origin is defined by

$$
\mathbb{E}\left[X^{n}\right]=\int_{\mathcal{D}(X)} x^{n} f_{X}(x) \mathrm{d} x, \quad n=0,1,2, \ldots,
$$

whenever the following integral exists and satisfies 


$$
\int_{\mathcal{D}(X)}|x|^{n} f_{X}(x) \mathrm{d} x<+\infty
$$

Symbol $\mathbb{E}[\cdot]$ in $(2.4)$ is usually called expectation operator. The first moment $\mathbb{E}[X]$ is particularly important because it gives the statistical average of $\mathrm{RV} X$ and usually it is referred to as mean, mathematical expectation or statistical average. In the following, it will be denoted by $\mu_{X}$ or $\mathbb{E}[X]$, indistinctly.

An important feature about existence of the moments with respect to origin is given in the following result

$$
\text { If } \mathbb{E}\left[|X|^{p}\right]<+\infty \text {, then } \mathbb{E}\left[X^{q}\right]<+\infty, \forall q \leq p,
$$

where the term $\mathbb{E}\left[|X|^{p}\right]$ is called the $p$-th absolute moment of $\mathrm{RV} X$ with respect to origin. Hereinafter, the existence of the mean of a RV will be assumed.

Given a collection of RVs, $X_{1}, X_{2}, \ldots, X_{n}$, and constants $a_{1}, a_{2}, \ldots, a_{n}$, the expectation operator is linear, that is, it satisfies

$$
\mathbb{E}\left[\sum_{i=1}^{n} a_{i} X_{i}\right]=\sum_{i=1}^{n} a_{i} \mathbb{E}\left[X_{i}\right] .
$$

The central moments of a $\mathrm{RV} X$ are the moments of $X$ with respect to its mean, $\mu_{X}$. Hence, the $n$-th central moment of $X$ is defined as

$$
\mathbb{E}\left[\left(X-\mu_{X}\right)^{n}\right]=\int_{\mathcal{D}(X)}\left(x-\mu_{X}\right)^{n} f_{X}(x) \mathrm{d} x, \quad n=0,1,2, \ldots
$$

The second central moment measures the spread or dispersion of RV $X$ about its mean. It is usually called variance, and in the following, it will be denoted by $\sigma_{X}^{2}$ or $\mathbb{V}[X]$, interchangeably. This statistical parameter can be expressed in terms of the first and second moments with respect to origin as follows

$$
\mathbb{V}[X]=\mathbb{E}\left[(X-\mathbb{E}[X])^{2}\right]=\mathbb{E}\left[X^{2}\right]-(\mathbb{E}[X])^{2} .
$$


In practice, the dispersion of a $\mathrm{RV} X$ is given in the same units that $\mathrm{RV} X$. It is done by means the square root of the variance, which is termed standard deviation, and it will be denoted by $\sigma_{X}$.

Tables 2.1 and 2.2 collect, respectively, the mean, the variance and, the moments with respect to origin of some relevant RVs that will appear throughout this thesis.

\begin{tabular}{cccc}
\hline Distribution & Notation & Mean & Variance \\
\hline \hline Uniform & $X \sim \operatorname{Un}([a, b]), a, b \in \mathbb{R}$ & $\frac{a+b}{2}$ & $\frac{(b-a)^{2}}{12}$ \\
\hline Beta & $X \sim \operatorname{Be}(\alpha ; \beta), \alpha, \beta>0$ & $\frac{\alpha}{\alpha+\beta}$ & $\frac{\alpha \beta}{(\alpha+\beta)^{2}(\alpha+\beta+1)}$ \\
\hline Gaussian & $X \sim \mathrm{N}(\mu ; \sigma), \sigma>0, \mu \in \mathbb{R}$ & $\mu$ & $\sigma^{2}$ \\
\hline Exponential & $X \sim \operatorname{Exp}(\lambda), \lambda>0$ & $\frac{1}{\lambda}$ & $\frac{1}{\lambda^{2}}$ \\
\hline Gamma & $X \sim \operatorname{Ga}(r ; a), \quad r, a>0$ & $\frac{r}{a}$ & $\frac{r}{a^{2}}$ \\
\hline
\end{tabular}

Table 2.1: Some relevant RVs and their mean and variance.

It is worthwhile pointing out that an Exponential RV $X \sim \operatorname{Exp}(\lambda)$ is a special case of a Gamma RV of parameters $r=1$ and $a=\lambda$. If $\alpha=\beta=1$, a Beta RV becomes a Uniform RV on interval $[0,1]$.

The transformation of a RV into another one (or the transformation of a random vector) will play a central role throughout this thesis. For resulting RV, it is convenient to have a direct way to compute its statistical properties, like expectation or variance, in terms of the information available for the original RV. The following result shows how can be calculated the mean of a function 
Distribution

\begin{tabular}{ccc}
\hline \hline Uniform & $X \sim \operatorname{Un}([0,1])$ & $\frac{1}{n+1}$ \\
\hline Beta & $X \sim \operatorname{Be}(\alpha ; \beta), \alpha, \beta>0$ & $\frac{(\alpha+n-1) !(\alpha+\beta-1) !}{(\alpha+\beta+n-1) !(\alpha-1) !}$ \\
\hline Gaussian & $X \sim \mathrm{N}(0 ; \sigma), \sigma>0$ & 0 if $n=2 k+1, \frac{(2 k) !}{2^{k} k !} \sigma^{2 k}$ if $n=2 k$ \\
\hline Exponential & $X \sim \operatorname{Exp}(\lambda), \lambda>0$ & $\frac{n !}{\lambda^{n}}$ \\
\hline Gamma & $X \sim \operatorname{Ga}(r ; a), \quad r, a>0$ & $\frac{(r+n-1) !}{a^{n}(r-1) !}$
\end{tabular}

Table 2.2: Some relevant RVs and their moments with respect to origin.

of a continuous RV $X$, say $g(X)$, with $\operatorname{PDF} f_{X}(x)$, without first determining its distribution

$$
\mathbb{E}[g(X)]=\int_{\mathcal{D}(X)} g(x) f_{X}(x) \mathrm{d} x .
$$

An analogous expression can be written for the discrete case replacing the integral by a sum.

In particular, if $g(x)=x$, expression (2.8) becomes the mean $\mathbb{E}[X]$ and, if $g(x)=x^{2}$, taking into account (2.7), one can obtain the variance $\sigma_{X}^{2}$.

The moments of two or more RVs are defined in a similar way. For the case of two RVs $X, Y$, the joint moments of order $(n, m)$ with respect to origin are defined by

$$
\mathbb{E}\left[X^{n} Y^{m}\right]=\int_{\mathcal{D}(Y)} \int_{\mathcal{D}(X)} x^{n} y^{m} f_{X Y}(x, y) \mathrm{d} x \mathrm{~d} y, \quad n, m=0,1,2, \ldots,
$$


whenever they exist. In the particular case that $X$ and $Y$ are independent RVs, by (2.3) one gets

$$
\begin{aligned}
\mathbb{E}\left[X^{n} Y^{m}\right] & =\int_{\mathcal{D}(Y)} \int_{\mathcal{D}(X)} x^{n} y^{m} f_{X}(x) f_{Y}(y) \mathrm{d} x \mathrm{~d} y \\
& =\left(\int_{\mathcal{D}(X)} x^{n} f_{X}(x) \mathrm{d} x\right)\left(\int_{\mathcal{D}(Y)} y^{m} f_{Y}(y) \mathrm{d} y\right) \\
& =\mathbb{E}\left[X^{n}\right] \mathbb{E}\left[Y^{m}\right] .
\end{aligned}
$$

The joint central moments of RVs $X$ and $Y$ of order $(n, m), n, m=0,1,2, \ldots$, are given by

$\mathbb{E}\left[(X-\mathbb{E}[X])^{n}(Y-\mathbb{E}[Y])^{m}\right]=\int_{\mathcal{D}(Y)} \int_{\mathcal{D}(X)}\left(x-\mu_{X}\right)^{n}\left(y-\mu_{Y}\right)^{m} f_{X Y}(x, y) \mathrm{d} x \mathrm{~d} y$.

The particular case

$$
\mathbb{E}[(X-\mathbb{E}[X])(Y-\mathbb{E}[Y])]=\mathbb{E}[X Y]-\mathbb{E}[X] \mathbb{E}[Y]
$$

is called covariance between $X$ and $Y$ and, in advance, it will be denoted by $\operatorname{Cov}[X, Y]$. The term $\mathbb{E}[X Y]$ is usually referred to as correlation between $X$ and $Y$. In this case, its covariance coincides with its correlation, whenever the second moment of RV X exists. From (2.10), if $X=Y$ then it is seen that covariance becomes the variance of $X$. Covariance, $\mathbb{C o v}[X, Y]$, has great importance in the statistical analysis of two RVs $X, Y$ because it provides a measure of their statistical linear interdependency in sense that its value is a measure of accuracy with which one RV can be approximated by a linear function of the other [85, p.18].

Two RVs $X$ and $Y$ are said to be uncorrelated if $\operatorname{Cov}[X, Y]=0$ or equivalently from (2.10) if $\mathbb{E}[X Y]=\mathbb{E}[X] \mathbb{E}[Y]$. Note that if $X$ and $Y$ are independent RVs with finite second moments with respect to origin, then they are uncorrelated, however the converse of this statement is not true in general, but an important exception is the case where $X$ and $Y$ are Gaussian RVs. 
In closing this section a set of inequalities, involving the moments with respect to origin of RVs, that will be used throughout this thesis are collected. If $X$ and $Y$ are RVs with finite second moments, then Schwarz inequality assures

$$
\mathbb{E}[|X Y|] \leq\left(\mathbb{E}\left[X^{2}\right]\right)^{1 / 2}\left(\mathbb{E}\left[Y^{2}\right]\right)^{1 / 2}
$$

A generalization of Schwarz inequality is known as Hölder inequality

$$
\mathbb{E}[|X Y|] \leq \mathbb{E}\left[|X|^{n}\right]^{1 / n} \mathbb{E}\left[|Y|^{m}\right]^{1 / m}, \quad n, m>1: \frac{1}{n}+\frac{1}{m}=1
$$

Let $f$ be a convex function on $\mathbb{R}$. If $\mathbb{E}[|X|]$ and $\mathbb{E}[|f(X)|]$ are finite, then Jensen inequality assures

$$
f(\mathbb{E}[X]) \leq \mathbb{E}[f(X)]
$$

In the particular case that $f(x)=x^{2}$, Jensen inequality (2.12) guarantees that the variance given by $(2.7)$ is non-negative.

\subsubsection{Stochastic processes}

RVs are adequate for to describe results of random experiments which assume scalar or vector values in a given trial. In many physical applications, however, the outcomes of a random experiment are represented by functions depending upon a parameter. These outcomes are then described by a random function $X(t)$, where $t$ is a parameter assuming values in a reference set $\mathcal{T}$, usually called index set. A typical example of random experiments giving rise to random functions is found in the theory of Brownian motion, where each coordinate of a particle executing Brownian motion exhibits random behaviour as a function of time. Other examples of random functions appearing in physical contexts are thermal noise, earthquake motion, epidemics, etc.

A SP $\{X(t): t \in \mathcal{T}\}$ is a collection of RVs, that is, for each $t \in \mathcal{T}, X(t)$ is a $\mathrm{RV}$. Depending on the index set $\mathcal{T}$ one classifies the SP as follows: If $\mathcal{T}$ is a countable set, the SP is said to be a discrete-time SP. For instance, a sequence of RVs $\left\{X_{n}: n=0,1,2, \ldots\right\}$ is a discrete-time SP indexed by the non-negative integers. If $\mathcal{T}$ is an interval of real line, the $\mathrm{SP}$ is said to be a continuoustime SP. For instance, $\{X(t): t \geq 0\}$ is a continuous-time SP indexed by the non-negative real numbers. 
Note that, every SP is defined not only on an index set $\mathcal{T}$, but also on a probability space, say, $(\Omega, \mathcal{A}, \mathbb{P})$. Then, it is more correct to denote it as $\{X(t ; \omega): t \in \mathcal{T}, \omega \in \Omega\}$, that is, as a function of two variables, although the usual notation suppresses the probability space variable $\omega$ (sometimes called as hidden parameter) for convenience. For each fixed $t \in \mathcal{T}, X(t ; \cdot)$ denotes a RV on the probability space $(\Omega, \mathcal{A}, \mathbb{P})$; while for each fixed $\omega \in \Omega, X(\cdot ; \omega)$ corresponds to a real-valued function defined on $\mathcal{T}$. The latter is called a sample path, trajectory, or realization of the process. The theory of SPs relates these inherent random and sample path structures.

As it has been emphasized previously, at a fixed $t \in \mathcal{T}$, a SP $\{X(t): t \in \mathcal{T}\}$ is a RV. Hence, another characterization of a SP is to regard it as a family of RVs, say, $X\left(t_{1}\right), X\left(t_{2}\right), \ldots$, depending upon a parameter $t \in \mathcal{T}$. The totality of all RVs define the SP $\{X(t): t \in \mathcal{T}\}$. For discrete-time SPs, this set of RVs is finite or countably infinite. For continuous-time SPs, the number is of noncountable infinite. The mathematical description of a SP is equivalent to the description of an infinite and generally non-countable number of RVs. Then, a SP $\{X(t): t \in \mathcal{T}\}$ is defined by a family of joint DFs or PDFs, dealing with infinitely many RVs. More exactly, if to every finite set $\left\{t_{1}, t_{2}, \ldots, t_{n}\right\}$ of $t \in \mathcal{T}$, there corresponds a set of RVs $X_{1}=X\left(t_{1}\right), X_{2}=X\left(t_{2}\right), \ldots, X_{n}=X\left(t_{n}\right)$, have a well-defined joint probability DF

$$
\begin{aligned}
& F_{X_{1}, \ldots, X_{n}}\left(x_{1}, t_{1} ; x_{2}, t_{2} ; \ldots ; x_{n}, t_{n}\right) \\
& \quad=P\left(\left\{\omega \in \Omega: X_{1}(\omega) \leq x_{1} \cap X_{2}(\omega) \leq x_{2} \cap \ldots X_{n}(\omega) \leq x_{n}\right\}\right), n \geq 1,
\end{aligned}
$$

then this family of joint DF defines a SP $\{X(t): t \in \mathcal{T}\}$. Usually, the following shorten notation will be used

$$
F_{n}\left(x_{1}, t_{1} ; x_{2}, t_{2} ; \ldots ; x_{n}, t_{n}\right)=F_{X_{1}, \ldots, X_{n}}\left(x_{1}, t_{1} ; x_{2}, t_{2} ; \ldots ; x_{n}, t_{n}\right) .
$$

It is called the $n$-th DF of SP $\{X(t): t \in \mathcal{T}\}$. The collection of DFs is not arbitrary; they must satisfy the Kolmogorov compatibility conditions

i) For $m>n$ :

$$
F_{m}\left(x_{1}, t_{1} ; \ldots ; x_{n}, t_{n} ;+\infty, t_{n+1} ; \ldots ;+\infty, t_{m}\right)=F_{n}\left(x_{1}, t_{1} ; \ldots ; x_{n}, t_{n}\right) .
$$

ii) The joint DF given by (2.13) is invariant under an arbitrary permutation of the indexes $1,2, \ldots, n$, i.e., 


$$
F_{n}\left(x_{1}, t_{1} ; x_{2}, t_{2} ; \ldots ; x_{n}, t_{n}\right)=F_{n}\left(x_{i_{1}}, t_{i_{1}} ; x_{i_{2}}, t_{i_{2}} ; \ldots ; x_{i_{n}}, t_{i_{n}}\right)
$$

where $\left\{i_{1}, i_{2}, \ldots, i_{n}\right\}$ is an arbitrary permutation of $\{1,2, \ldots, n\}$.

Given the $n$-th DF $F_{n}\left(x_{1}, t_{1} ; x_{2}, t_{2} ; \ldots ; x_{n}, t_{n}\right)$ of an arbitrary $\operatorname{SP}\{X(t): t \in \mathcal{T}\}$, its associated $n$-th joint PDF, assuming that it exists, is defined as

$$
f_{n}\left(x_{1}, t_{1} ; x_{2}, t_{2} ; \ldots ; x_{n}, t_{n}\right)=\frac{\partial^{n} F_{X_{1}, \ldots, X_{n}}\left(x_{1}, t_{1} ; \ldots ; x_{n}, t_{n}\right)}{\partial x_{1} \cdots \partial x_{n}}
$$

As in the case of RVs, some of the most important properties of a SP are characterized by its moments, mainly, those of first and second order. In the sequel, existence of DF shall be assumed. In terms of its first PDF (1-PDF), $f_{1}(x, t)$, the $n$-th moment of a SP $\{X(t): t \in \mathcal{T}\}$ at a fixed $t \in \mathcal{T}$ is defined by

$$
\mathbb{E}\left[(X(t))^{n}\right]=\int_{\mathcal{D}(X)} x^{n} f_{1}(x, t) \mathrm{d} x
$$

The first moment represents the mean of the SP at $t$. It will be sometimes denoted by $\mu_{X}(t)$ or $\mathbb{E}[X(t)] . \mathbb{E}\left[X(t)^{2}\right]$ represents the mean square value of $\mathrm{SP}$ at $t$.

The $n$-th central moment of $\{X(t): t \in \mathcal{T}\}$, at a given $t \in \mathcal{T}$, is

$$
\mathbb{E}\left[\left(X(t)-\mu_{X}(t)\right)^{n}\right]=\int_{\mathcal{D}(X)}\left(x-\mu_{X}(t)\right)^{n} f_{1}(x, t) \mathrm{d} x .
$$

Of particular interest is when $n=2$, that represents the variance of SP at $t$, and it will be denoted by $\sigma_{X}^{2}(t)$ or $\mathbb{V}[X(t)]$.

\subsubsection{Mean square calculus}

As it was pointed at the introduction, in this dissertation random difference and differential equations will be studied. In dealing with this goal it can be advisable the use of mean square (m.s.) calculus. Although in this thesis the problem will be focused from another point of view, it is necessary to recall some concepts and notations related to it that may be found in [85]. Let $(\Omega, \mathcal{A}, \mathbb{P})$ be a probability space, here it will be considered real $\operatorname{RVs} X: \Omega \rightarrow \mathbb{R}$ 
whose second-order moment with respect to origin is finite, that is, $\mathbb{E}\left[X^{2}\right]<\infty$, called second-order RVs (2-RVs). From Schwarz inequality (2.11) and property (2.6) it follows directly

$$
\mathbb{E}\left[(X+Y)^{2}\right]<\infty, \quad \mathbb{E}\left[(c X)^{2}\right]=c^{2} \mathbb{E}\left[X^{2}\right]<\infty, \forall c \in \mathbb{R}
$$

Hence the class of all second order RVs on a probability space constitutes a real linear space if all equivalent RVs are identified by the following equivalence binary relationship: two RVs $X$ and $Y$ are said to be equivalent if, and only if, $\mathbb{P}[X \neq Y]=0$.

Defining

$$
\langle X, Y\rangle=\mathbb{E}[X Y]
$$

by Schwarz inequality (2.11) and Jensen inequality (2.12), one gets $|\langle X, Y\rangle|<$, $\infty$. It is easy to prove that \langle\rangle given by (2.14) defines an inner product when $X$ and $Y$ are 2-RVs. This inner product automatically defines a norm

$$
\|X\|_{2}=+\sqrt{\langle X, X\rangle}=\left(\mathbb{E}\left[X^{2}\right]\right)^{1 / 2},
$$

and a metric

$$
\mathrm{d}(X, Y)=\|X-Y\|_{2}=+\sqrt{\mathbb{E}\left[(X-Y)^{2}\right]} .
$$

The linear vector space of 2-RVs with this inner product, the norm, and the distance defined above is called $\mathrm{L}_{2}$-space. The convergence that above norm defines in $\mathrm{L}_{2}$ is referred to as m.s. convergence. A sequence of 2-RVs $\left\{X_{n}: n \geq 0\right\}$ is m.s. convergent to $X$ if

$$
\lim _{n \rightarrow \infty}\left\|X_{n}-X\right\|_{2}=\lim _{n \rightarrow \infty}\left(\mathbb{E}\left[\left(X_{n}-X\right)^{2}\right]\right)^{1 / 2}=0 .
$$

A sequence $\left\{X_{n}: n \geq 0\right\}$ in $\mathrm{L}_{2}$ is said to be a Cauchy sequence in m.s. sense if

$$
\left\|X_{n}-X_{m}\right\|_{2} \rightarrow 0, \quad \text { as } n, m \rightarrow \infty .
$$


It can be proved that a sequence $\left\{X_{n}: n \geq 0\right\}$ of $2-\mathrm{RVs}$ is m.s. convergent to a RV $X$ as $n \rightarrow \infty$ if, and only if, is a Cauchy sequence.

\subsection{Random Variable Transformation method}

As it was pointed in the introduction, the main result that will be used in this thesis is the Random Variable Transformation (RVT) technique. This method states as follows in its general form.

Theorem 2.1 (Multidimensional RVT method, [85]) Let us consider $\mathbf{X}=\left(X_{1}, \ldots, X_{m}\right)$ and $\mathbf{Y}=\left(Y_{1}, \ldots, Y_{m}\right)$ two $m$-dimensional absolutely continuous random vectors defined on a complete probability space $(\Omega, \mathcal{A}, \mathbb{P})$. Let $\mathbf{r}: \mathbb{R}^{m} \rightarrow \mathbb{R}^{m}$ be a one-to-one deterministic transformation of $\mathbf{X}$ into $\mathbf{Y}$, i.e., $\mathbf{Y}=\mathbf{r}(\mathbf{X})$. Assume that $\mathbf{r}$ is continuous in $\mathbf{X}$ and has continuous partial derivatives with respect to $\mathbf{X}$. Then, if $f_{\mathbf{X}}(\mathbf{x})$ denotes the joint probability density function of vector $\mathbf{X}$, and $\mathbf{s}=\mathbf{r}^{-1}=\left(s_{1}\left(y_{1}, \ldots, y_{m}\right), \ldots, s_{m}\left(y_{1}, \ldots, y_{m}\right)\right)$ represents the inverse mapping of $\mathbf{r}=\left(r_{1}\left(x_{1}, \ldots, x_{m}\right), \ldots, r_{m}\left(x_{1}, \ldots, x_{m}\right)\right)$, the joint probability density function of vector $\mathbf{Y}$ is given by

$$
f_{\mathbf{Y}}(\mathbf{y})=f_{\mathbf{X}}(\mathbf{s}(\mathbf{y}))\left|J_{m}\right|
$$

where $\left|J_{m}\right|$, which is assumed to be different from zero, denotes the absolute value of the Jacobian defined by the determinant

$$
J_{m}=\operatorname{det}\left(\begin{array}{ccc}
\frac{\partial s_{1}\left(y_{1}, \ldots, y_{m}\right)}{\partial y_{1}} & \ldots & \frac{\partial s_{m}\left(y_{1}, \ldots, y_{m}\right)}{\partial y_{1}} \\
\vdots & \ddots & \vdots \\
\frac{\partial s_{1}\left(y_{1}, \ldots, y_{m}\right)}{\partial y_{m}} & \cdots & \frac{\partial s_{m}\left(y_{1}, \ldots, y_{m}\right)}{\partial y_{m}}
\end{array}\right)
$$

Now, several specializations of RVT technique, Theorem 2.1, that will be required throughout this dissertation, will be established. In particular, Propositions 2.2-2.6 are applied in Chapter 3; Propositions 2.6 and 2.7 in Chapter 4; and Propositions 2.8 and 2.9 in Chapter 5. 
Proposition 2.2 Let $U$ be an absolutely continuous real $R V$ defined on a complete probability space $(\Omega, \mathcal{A}, \mathbb{P})$, with $P D F f_{U}(u)$ such that $U(\omega) \neq 0, \forall \omega \in \Omega$. Then, the PDF $f_{V}(v)$ of the inverse transformation $V=\frac{1}{U}$ is given by

$$
f_{V}(v)=\frac{1}{v^{2}} f_{U}\left(\frac{1}{v}\right)
$$

Proof. Let us consider the mapping $v=r(u)=\frac{1}{u}$. Its inverse mapping exists and is given by $u=s(v)=\frac{1}{v}$, being its derivative $s^{\prime}(v)=-\frac{1}{v^{2}}$. Then, by applying RVT technique, Theorem 2.1, with the identification $X=U, Y=V$, $m=1$ and $\left|J_{1}\right|=1 / v^{2}$, which is nonzero and is well defined as $U \neq 0$ a.s., the expression (2.18) is straightforwardly obtained.

Proposition 2.3 Let $\mathbf{U}=\left(U_{1}, U_{2}\right)$ be an absolutely continuous real random vector defined on a complete probability space $(\Omega, \mathcal{A}, \mathbb{P})$, with joint $P D F f_{\mathbf{U}}\left(u_{1}, u_{2}\right)$ such that $U_{1}(\omega) \neq 0, \forall \omega \in \Omega$. Then, the joint PDF $f_{\mathbf{V}}\left(v_{1}, v_{2}\right)$ of the inverseopposite transformation $\mathbf{V}=\left(V_{1}, V_{2}\right)=\left(\frac{1}{U_{1}},-U_{2}\right)$ is given by

$$
f_{\mathbf{V}}\left(v_{1}, v_{2}\right)=\frac{1}{\left(v_{1}\right)^{2}} f_{\mathbf{U}}\left(\frac{1}{v_{1}},-v_{2}\right) \text {. }
$$

Proof. Let us consider the two-dimensional transformation $\left(v_{1}, v_{2}\right)=\mathbf{r}\left(u_{1}, u_{2}\right)$ $=\left(1 / u_{1},-u_{2}\right)$. Notice that its inverse mapping is given by $\left(u_{1}, u_{2}\right)=\mathbf{s}\left(v_{1}, v_{2}\right)=$ $\left(1 / v_{1},-v_{2}\right)$, being its Jacobian

$$
J_{2}=\operatorname{det}\left(\begin{array}{cc}
-\frac{1}{\left(v_{1}\right)^{2}} & 0 \\
0 & -1
\end{array}\right)=\frac{1}{\left(v_{1}\right)^{2}} \neq 0
$$

which is well defined since $V_{1}=1 / U_{1}$ and $U_{1} \neq 0$ a.s. Then, applying RVT technique, Theorem 2.1, for $m=2$ and the identification $X_{i}=U_{i}, Y_{i}=V_{i}$, $i=1,2$, the expression (2.19) is straightforwardly obtained.

Proposition 2.4 Let $\mathbf{U}=\left(U_{1}, U_{2}\right)$ be an absolutely continuous real random vector defined on a complete probability space $(\Omega, \mathcal{A}, \mathbb{P})$, with joint $P D F f_{\mathbf{U}}\left(u_{1}, u_{2}\right)$. 
Then, the joint PDF $f_{\mathbf{V}}\left(v_{1}, v_{2}\right)$ of the opposite-opposite transformation $\mathbf{V}=$ $\left(V_{1}, V_{2}\right)=\left(-U_{1},-U_{2}\right)$ is given by

$$
f_{\mathbf{V}}\left(v_{1}, v_{2}\right)=f_{\mathbf{U}}\left(-v_{1},-v_{2}\right)
$$

Proof. Let us consider the two-dimensional transformation $\left(v_{1}, v_{2}\right)=\mathbf{r}\left(u_{1}, u_{2}\right)$ $=\left(-u_{1},-u_{2}\right)$. Notice that its inverse mapping is given by $\left(u_{1}, u_{2}\right)=\mathbf{s}\left(v_{1}, v_{2}\right)=$ $\left(-v_{1},-v_{2}\right)$, being its Jacobian

$$
J_{2}=\operatorname{det}\left(\begin{array}{cc}
-1 & 0 \\
0 & -1
\end{array}\right)=1 \neq 0
$$

Then, applying RVT technique, Theorem 2.1, for $m=2$ and the identification $X_{i}=U_{i}, Y_{i}=V_{i}, i=1,2$, the expression (2.20) follows straightforwardly.

Proposition 2.5 Let $\mathbf{U}=\left(U_{1}, U_{2}, U_{3}\right)$ be an absolutely continuous real random vector defined on a complete probability space $(\Omega, \mathcal{A}, \mathbb{P})$, with joint PDF $f_{\mathbf{U}}\left(u_{1}, u_{2}, u_{3}\right)$ such that $U_{1}(\omega) \neq 0, \forall \omega \in \Omega$. Then, the joint PDF $f_{\mathbf{V}}\left(v_{1}, v_{2}, v_{3}\right)$ of inverse-opposite-opposite transformation $\mathbf{V}=\left(V_{1}, V_{2}, V_{3}\right)=\left(1 / U_{1},-U_{2},-U_{3}\right)$ is given by

$$
f_{\mathbf{V}}\left(v_{1}, v_{2}, v_{3}\right)=\frac{1}{\left(v_{1}\right)^{2}} f_{\mathbf{U}}\left(1 / v_{1},-v_{2},-v_{3}\right) .
$$

Proof. Let us consider the three-dimensional transformation $\left(v_{1}, v_{2}, v_{3}\right)=$ $\mathbf{r}\left(u_{1}, u_{2}, u_{3}\right)=\left(1 / u_{1},-u_{2},-u_{3}\right)$. Notice that its inverse mapping is given by $\left(u_{1}, u_{2}, u_{3}\right)=\mathbf{s}\left(v_{1}, v_{2}, v_{3}\right)=\left(1 / v_{1},-v_{2},-v_{3}\right)$, being its Jacobian

$$
J_{3}=\operatorname{det}\left(\begin{array}{ccc}
-\frac{1}{\left(v_{1}\right)^{2}} & 0 & 0 \\
0 & -1 & 0 \\
0 & 0 & -1
\end{array}\right)=-\frac{1}{\left(v_{1}\right)^{2}} \neq 0
$$

which is well defined since $V_{1}=1 / U_{1}$ and $U_{1} \neq 0$ a.s. Then, applying RVT technique, Theorem 2.1, for $m=3$ and the identification $X_{i}=U_{i}, Y_{i}=V_{i}$, $i=1,2,3$, the expression (2.21) follows straightforwardly. 
Proposition 2.6 Let $U$ be an absolutely continuous real $R V$ defined on a complete probability space $(\Omega, \mathcal{A}, \mathbb{P})$, with PDF $f_{U}(u)$. Then, the PDF $f_{V}(v)$ of the linear transformation $V=a U+b, a \neq 0$ is given by

$$
f_{V}(v)=\frac{1}{|a|} f_{U}\left(\frac{v-b}{a}\right)
$$

If $a=0$, then $V=b$ w.p. 1 and

$$
f_{V}(v)=\delta(v-b), \quad-\infty<v<+\infty,
$$

being $\delta(\cdot)$ the Dirac delta function.

Proof. Let us consider the mapping $v=r(u)=a u+b$ with $a \neq 0$. Its inverse mapping exists and is given by $u=s(v)=(v-b) / a$, being its derivative $s^{\prime}(v)=$ $1 / a$. Then, applying RVT technique, Theorem 2.1, with the identification $X=U, Y=V, m=1$ and $\left|J_{1}\right|=|1 / a| \neq 0$, which is well defined since $a \neq 0$, the expression (2.22) is straightforwardly obtained. If $a=0, \mathrm{RV} V$ takes the punctual value $b$ a.s., then by expression (2.2) the PDF is obtained.

Proposition 2.7 Let $\mathbf{U}=\left(U_{1}, U_{2}\right)$ be an absolutely continuous real random vector defined on a complete probability space $(\Omega, \mathcal{A}, \mathbb{P})$, with joint $P D F f_{\mathbf{U}}\left(u_{1}, u_{2}\right)$ such that $U_{1}(\omega) \neq 0, \forall \omega \in \Omega$. Then, the PDF $f_{V}(v)$ of the quotient $V=U_{2} / U_{1}$ is given by

$$
f_{V}(v)=\int_{\mathcal{D}\left(U_{1}\right)} f_{U_{1}, U_{2}}(\xi, v \xi)|\xi| \mathrm{d} \xi
$$

where $\mathcal{D}\left(U_{1}\right)$ denotes the domain of $R V U_{1}$.

Proof. Let us consider the RV $V_{1}=U_{2} / U_{1}$. Then, introducing the auxiliary RV $V_{2}=U_{1}$ and denoting by $\mathbf{V}=\left(V_{1}, V_{2}\right)$, one can define the mapping $\left(v_{1}, v_{2}\right)=\mathbf{r}\left(u_{1}, u_{2}\right)=\left(u_{2} / u_{1}, u_{1}\right)$. Notice that its inverse mapping is given by $\left(u_{1}, u_{2}\right)=\mathbf{s}\left(v_{1}, v_{2}\right)=\left(v_{1} v_{2}, v_{2}\right)$, being its Jacobian

$$
J_{2}=\operatorname{det}\left(\begin{array}{cc}
0 & 1 \\
v_{2} & v_{1}
\end{array}\right)=-v_{2} \neq 0 \text {. }
$$


Notice that $v_{2} \neq 0$ since $V_{2}=U_{1}$, which by hypothesis is nonzero a.s. Then, by applying RVT technique, Theorem 2.1, for $m=2$ and the identification $X_{i}=U_{i}, Y_{i}=V_{i}, i=1,2$, the joint $\mathrm{PDF}$ of random vector $\mathbf{V}=\left(V_{1}, V_{2}\right)$ is given by

$$
f_{V_{1}, V_{2}}\left(v_{1}, v_{2}\right)=f_{U_{1}, U_{2}}\left(v_{2}, v_{1} v_{2}\right)\left|v_{2}\right|
$$

Hence, the PDF of the quotient $V_{1}=U_{2} / U_{1}$ is obtained as the $V_{2}$-marginal $\mathrm{PDF}$ of $f_{V_{1}, V_{2}}\left(v_{1}, v_{2}\right)$, i.e.,

$$
f_{V_{1}}\left(v_{1}\right)=\int_{\mathcal{D}\left(U_{1}\right)} f_{U_{1}, U_{2}}\left(u_{1}, v_{1} u_{1}\right)\left|u_{1}\right| \mathrm{d} u_{1}
$$

where $\mathcal{D}\left(U_{1}\right)$ denotes the domain of RV $U_{1}$, and the relationship $V_{2}=U_{1}$ has been used.

Proposition 2.8 Let $\mathbf{U}=\left(U_{1}, U_{2}\right)$ be an absolutely continuous real random vector defined on a complete probability space $(\Omega, \mathcal{A}, \mathbb{P})$, with joint $P D F f_{\mathbf{U}}\left(u_{1}, u_{2}\right)$ such that $U_{1}(\omega)>0$ and $U_{2}(\omega) \neq 1, \forall \omega \in \Omega$. Then, the PDF $f_{W}(w)$ of the transformation $W=\left(U_{1}\right)^{\frac{1}{1-U_{2}}}$ is given by

$$
f_{W}(w)=\int_{\mathcal{D}\left(U_{2}\right)} f_{U_{1}, U_{2}}\left(w^{1-u_{2}}, u_{2}\right)\left|\left(1-u_{2}\right) w^{-u_{2}}\right| \mathrm{d} u_{2} .
$$

where $\mathcal{D}\left(U_{2}\right)$ denotes the domain of $R V U_{2}$.

Proof. Let us consider the RV $V_{1}=\left(U_{1}\right)^{\frac{1}{1-U_{2}}}$. Then, introducing the auxiliary $\mathrm{RV} V_{2}=U_{2}$ and denoting by $\mathbf{V}=\left(V_{1}, V_{2}\right)$, one can define the mapping $\left(v_{1}, v_{2}\right)=\mathbf{r}\left(u_{1}, u_{2}\right)=\left(\left(u_{1}\right)^{\frac{1}{1-u_{2}}}, u_{2}\right)$. Notice that its inverse mapping is given by $\left(u_{1}, u_{2}\right)=\mathbf{s}\left(v_{1}, v_{2}\right)=\left(\left(v_{1}\right)^{1-v_{2}}, v_{2}\right)$, being its Jacobian

$$
J_{2}=\operatorname{det}\left(\begin{array}{cc}
\left(1-v_{2}\right)\left(v_{1}\right)^{-v_{2}} & -\left(v_{1}\right)^{1-v_{2}} \log \left(v_{1}\right) \\
0 & 1
\end{array}\right)=\left(1-v_{2}\right)\left(v_{1}\right)^{-v_{2}} \neq 0 .
$$

Notice that $J_{2} \neq 0$ because by hypothesis $U_{1}(\omega)>0$ and $U_{2}(\omega) \neq 1$ w.p. 1 . Then, applying RVT technique, Theorem 2.1, for $m=2$ and the identification 
$X_{i}=U_{i}, Y_{i}=V_{i}, i=1,2$, the joint PDF of random vector $\mathbf{V}=\left(V_{1}, V_{2}\right)$ is given by

$$
f_{V_{1}, V_{2}}\left(v_{1}, v_{2}\right)=f_{U_{1}, U_{2}}\left(\left(v_{1}\right)^{1-v_{2}}, v_{2}\right)\left|\left(1-v_{2}\right)\left(v_{1}\right)^{-v_{2}}\right|
$$

Hence, the PDF of RV $W=V_{1}$ is obtained as the $V_{2}$-marginal PDF of $f_{V_{1}, V_{2}}\left(v_{1}, v_{2}\right)$, i.e.,

$$
f_{W}(w)=\int_{\mathcal{D}\left(U_{2}\right)} f_{U_{1}, U_{2}}\left(w^{1-u_{2}}, u_{2}\right)\left|\left(1-u_{2}\right) w^{-u_{2}}\right| \mathrm{d} u_{2},
$$

where $\mathcal{D}\left(U_{2}\right)$ denotes the domain of $U_{2}$ and the relationship $V_{2}=U_{2}$ has been used.

Proposition 2.9 Let $Z: \Omega \rightarrow \mathbb{R}$ be an absolutely continuous real random variable defined on a complete probability space $(\Omega, \mathcal{A}, \mathbb{P})$, with probability density function $f_{Z}(z)$. Assume that $Z(\omega) \neq 0$ for all $\omega \in \Omega$. Then, the probability density function $f_{W}(w)$ of the transformation $W=Z^{3}$ is given by

$$
f_{W}(w)=\frac{1}{3} f_{Z}(\sqrt[3]{w})|w|^{-2 / 3} .
$$

Proof.This result is a direct consequence of Theorem 2.1 for $n=1, \mathbf{X}=Z$, $\mathbf{Y}=W$ and $W=r(Z)=Z^{3}$. Notice that the inverse transformation of $r$ is $s(w)=\sqrt[3]{w}$ and its Jacobian is given by $h^{\prime}(w)=1 / 3 w^{-2 / 3}$. It is well-defined because $Z(\omega) \neq 0$ w.p. 1 , by hypothesis. Then, applying (2.17) one obtains directly expression $(2.25)$. 


\section{Chapter 3}

\section{Random homogeneous Riccati differential equation}

This chapter deals with the determination of the first probability density function of the solution stochastic process to homogeneous Riccati random differential equation taking advantage of both linearization and Random Variable Transformation techniques. The study is split in all possible cases regarding the deterministic/random character of the involved input parameters. An illustrative example is provided for each one of the considered cases.

\subsection{Introduction}

The aim of this chapter is to compute the 1-PDF of the solution SP of the following random IVP based on a homogeneous Riccati-type differential equation

$$
\left.\begin{array}{l}
\dot{X}(t)=C X(t)+D(X(t))^{2}, \quad t \geq 0, \\
X(0)=X_{0},
\end{array}\right\}
$$

where all input parameters $X_{0}, C$ and $D$ are assumed to be absolutely continuous RVs defined on a common complete probability space, $(\Omega, \mathcal{A}, \mathbb{P})$. Their PDFs will be denoted by $f_{X_{0}}\left(x_{0}\right), f_{C}(c)$, and $f_{D}(d)$, respectively. Hereinafter, $\mathcal{D}\left(X_{0}\right), \mathcal{D}(C)$ and $\mathcal{D}(D)$, will represent their respective domains. For sake 
of generality, statistical dependence among input RVs $X_{0}, D$ and $C$ will be also considered. In such case, functions $f_{X_{0}, D}\left(x_{0}, d\right), f_{X_{0}, C}\left(x_{0}, c\right), f_{D, C}(d, c)$ and $f_{X_{0}, D, C}\left(x_{0}, d, c\right)$, will denote the joint PDFs of random vectors $\left(X_{0}, D\right)$, $\left(X_{0}, C\right),(D, C)$ and $\left(X_{0}, D, C\right)$, respectively. Since input parameters can be deterministic or random, in the following they will be distinguish by writing deterministic variables by lower cases and RVs by upper cases. In this way, if non-linear coefficient in (3.1) is deterministic, then it will be denoted as $d$, whereas $D$ will mean that it is a RV.

The study of this IVP has interest by itself from a mathematical standpoint since it constitutes the extension of homogeneous Riccati differential equation to random scenario. In addition, this differential equation arises frequently in important applications to classical control problems, as decoupling techniques for both analytic and numerical study of boundary value problems [4, 76], and also, for instance, in dealing with SI-type epidemiological models [11]. Therefore, its generalization to random framework can be very useful in order to develop more accurate models that consider uncertainty usually involved in real phenomena. Notice that, in the stochastic context, [32] deal with random Riccati differential equations. In that paper, coefficients are assumed to be analytic SPs and taking advantage of $\mathrm{L}_{p}$-calculus approximate solutions for the mean and the variance of solution SP are constructed. However, in that contribution none information about the 1-PDF of solution SP is provided.

The chapter is organized as follows. Section 3.2 is devoted to solve the problem, that is, to compute explicit expressions for the 1-PDF of solution SP. The resolution will be split in several cases depending on the random nature of inputs parameters. Examples in each one of these cases are included to illustrate the theoretical results. Conclusions are drawn in Section 3.3.

\subsection{Probabilistic solution}

In order to determine the 1-PDF of solution SP of IVP (3.1), the results established in [16] will be applied, where a comprehensive study to compute the 1-PDF of linear random IVP

$$
\left.\begin{array}{rl}
\dot{Z}(t) & =A Z(t)+B, \quad t \geq t_{0} \\
Z\left(t_{0}\right) & =Z_{0}
\end{array}\right\}
$$


is provided. With this aim, notice that making the change of variable

$$
Z(t)=\frac{1}{X(t)}
$$

non-linear IVP (3.1) is transformed into linear IVP (3.2), using the following identification of random inputs

$$
Z_{0}=\frac{1}{X_{0}}, \quad B=-D, \quad A=-C
$$

and taking $t_{0}=0$. In this manner, all the results obtained in [16] are available.

In order to facilitate the comparison regarding the notation as well as the cases considered in [16] for IVP (3.2) with respect to the one to be used for IVP (3.1), an identification between both problems is shown in Table 3.1.

It is important to underline that Cases I.1-I.3, corresponding to the situation where non-linear coefficient $D=0$ w.p. 1, i.e., $\mathbb{P}[\{\omega \in \Omega: D(\omega)=0\}]=1$, will be omitted in the subsequent analysis since it was already studied in reference [16]. Specifically, it corresponds to random homogeneous linear differential equation given in $\operatorname{IVP}(3.2)$ taking $B=0$ w.p. 1, i.e., $\mathbb{P}[\{\omega \in \Omega: B(\omega)=0\}]=1$.

This section will be split in two parts, Subsection 3.2.1 and Subsection 3.2.2, where the Cases II.1-II-3 and Cases III.1-III.7 will be solved, respectively.

\subsubsection{Solving the Cases II.1-II.3}

This subsection is addressed to compute the 1-PDF, $f_{1}(x, t)$, of solution SP of IVP (3.1) in each one of the Cases II.1-II.3 collected in Table 3.1. Thus, throughout this subsection the deterministic parameter $c$ that appears into problem (3.1) is assumed to be null, $c=0$. As it was pointed out to conduct the analysis the results obtained in Cases II.1-II.3 studied in [16] (see IVP (3.2) in Table 3.1) will be applied. 
$\operatorname{IVP}(3.2)$

Case Random Deterministic

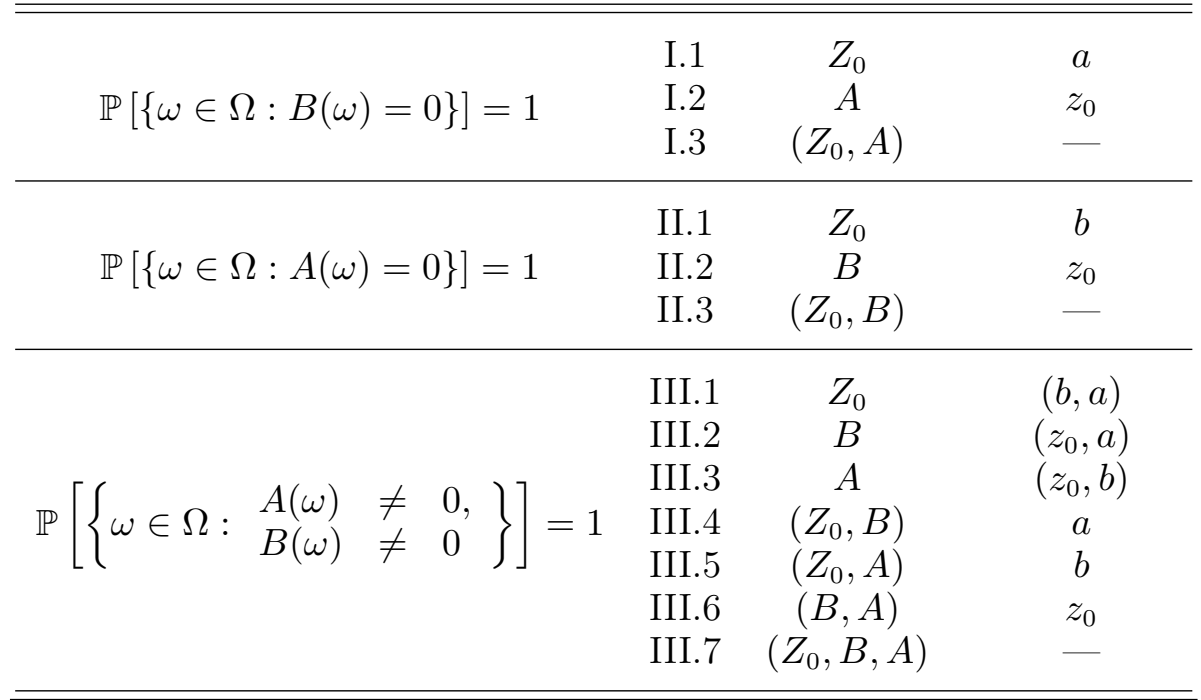

$\operatorname{IVP}(3.1)$

Case Random Deterministic

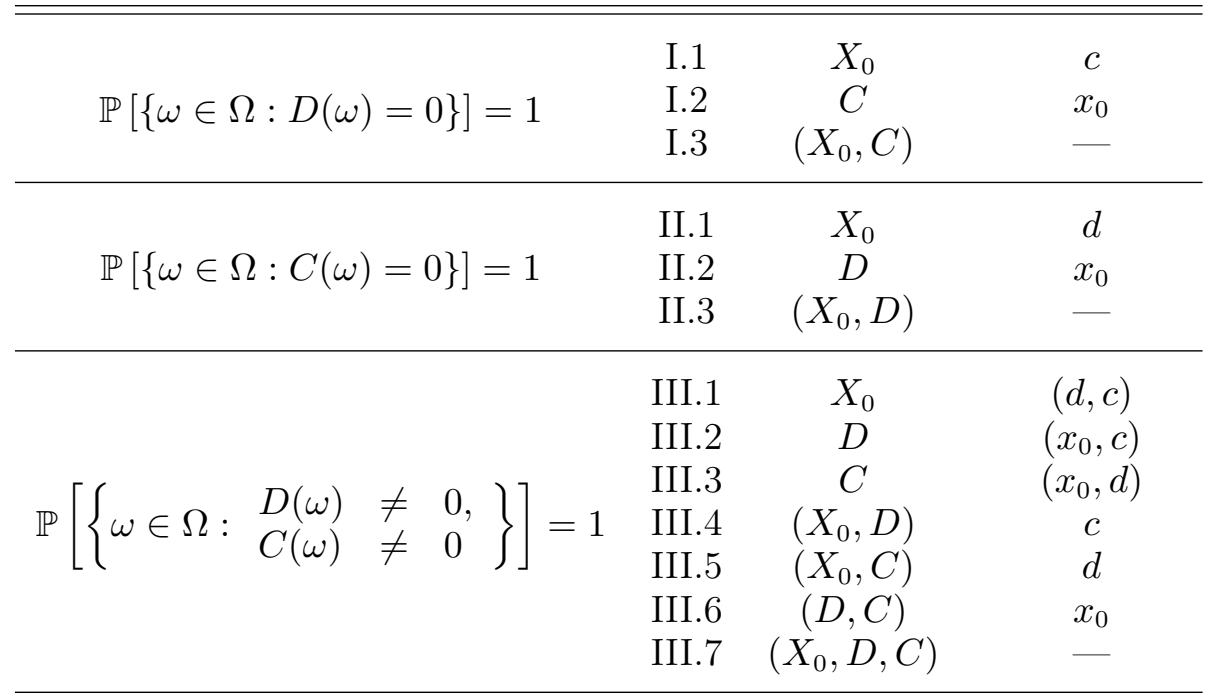

Table 3.1: List of different cases in which IVP (3.1) is split to conduct the study and identification for the notation used regarding the involved deterministic/random inputs in IVPs (3.2) and (3.1). 


\section{Case II.1: $\mathrm{X}_{0}$ is a random variable}

Notice that regarding the main problem (3.1), it is implicitly assumed that $d \in \mathbb{R}-\{0\}$ and $X_{0}$ is a RV with PDF $f_{X_{0}}\left(x_{0}\right)$. In accordance with Table 3.1 and identification (3.4), this situation corresponds to the following particular case of linear IVP (3.2)

$$
\left.\begin{array}{l}
\dot{Z}(t)=b, \\
Z(0)=Z_{0},
\end{array}\right\} \quad Z_{0}=\frac{1}{X_{0}}, \quad b=-d .
$$

Now, fix $t \geq 0$ and apply [16, Equation (59)] in order to compute the PDF of solution SP of IVP (3.5) evaluated at that $t$, since the randomness character of $X_{0}$ is transferred to $Z_{0}$

$$
f_{Z}(z)=f_{Z_{0}}(z-b t)
$$

Note that for the sake of clarity, the notation $f_{Z}(z)$ instead of $f_{1}(z, t)$ has been used, since the time variable $t$ has been fixed, so $Z=Z(t)$ is a RV rather than a SP.

Taking into account (3.5) and applying Proposition 2.2 to $U=X_{0}, V=Z_{0}$, formula (3.6) can be expressed in terms of data. This yields

$$
f_{Z}(z)=f_{Z_{0}}(z+d t)=\frac{1}{(z+d t)^{2}} f_{X_{0}}\left(\frac{1}{z+d t}\right) .
$$

Considering (3.3), which establishes the relationship between solutions of IVPs (3.1) and (3.2), $X(t)=1 / Z(t)$, and applying Proposition 2.2 to $U=Z$ and $V=X$, with $Z=Z(t)$ and $X=X(t)$, for each $t \geq 0$, one gets

$$
f_{X}(x)=\frac{1}{x^{2}} f_{Z}\left(\frac{1}{x}\right)=\frac{1}{x^{2}} \frac{1}{\left(\frac{1}{x}+d t\right)^{2}} f_{X_{0}}\left(\frac{1}{\frac{1}{x}+d t}\right) .
$$

Since $t \geq 0$ is arbitrary, this expression represents the 1-PDF of solution SP $X(t)$ of IVP $(3.1)$

$$
f_{1}(x, t)=\frac{1}{(1+d t x)^{2}} f_{X_{0}}\left(\frac{x}{1+d t x}\right), \quad t \geq 0 .
$$


Although the domains of the 1-PDFs that will be determined throughout this chapter could be specified in the same way they were done in [16], now they will be omitted because their specification become cumbersome. For instance, in the context of the current Case II.1, if $\mathcal{D}\left(X_{0}\right)$ denotes the domain of RV $X_{0}$, then the domain of the 1-PDF (3.7) can be determined by imposing that

$$
\frac{x}{1+d t x} \in \mathcal{D}\left(X_{0}\right) \text {. }
$$

This issue will be illustrated in the following example, where the domain of the 1-PDF is completely determined.

Example 3.1 Let us assume that $d=-1$ and $X_{0}$ has an Exponential distribution of parameter $\lambda=1$, i.e., $X_{0} \sim \operatorname{Exp}(1)$. Then, in accordance with (3.7) the 1-PDF to solution $S P X(t) \equiv X(t, \omega), \omega \in \Omega$, of IVP (3.1) is given by

$$
f_{1}(x, t)=\frac{1}{(1-t x)^{2}} \mathrm{e}^{-\frac{x}{1-t x}}, \quad t>0, \quad 0<x<\frac{1}{t} .
$$

For the full specification of the domain, observe that as $X_{0} \sim \operatorname{Exp}(1)$ and $t>0$ then, in accordance with (3.8)

$$
\frac{x}{1-t x}>0 \Longleftrightarrow x<\frac{1}{t}
$$

It is easy to check that $\int_{0}^{1 / t} f_{1}(t, x) \mathrm{d} x=1$. In Figure 3.1, $f_{1}(x, t)$ is represented for different values of $t$. Important statistical information associated to solution $S P X(t)$ can be determined from its $1-P D F$, such as, the mean, $\mathbb{E}[X(t)]$, and the variance, $\mathbb{V}[X(t)]$. Taking into account (3.9), the expectation is given by

$$
\mathbb{E}[X(t)]=\int_{-\infty}^{\infty} x f_{1}(x, t) \mathrm{d} x=\int_{0}^{1 / t} \frac{x}{(1-t x)^{2}} \mathrm{e}^{-\frac{x}{1-t x}} \mathrm{~d} x=\frac{t-\mathrm{e}^{\frac{1}{t}} \int_{1 / t}^{\infty} \mathrm{e}^{-\xi} / \xi \mathrm{d} \xi}{t^{2}} .
$$

In order to determine $\mathbb{V}[X(t)]$, first it is needed to compute

$$
\mathbb{E}\left[(X(t))^{2}\right]=\int_{0}^{1 / t} x^{2} f_{1}(x, t) \mathrm{d} x=\frac{t(1+t)-\mathrm{e}^{\frac{1}{t}}(1+2 t) \int_{1 / t}^{\infty} \mathrm{e}^{-\xi} / \xi \mathrm{d} \xi}{t^{4}} .
$$


Therefore

$$
\begin{aligned}
\mathbb{V}[X(t)] & =\mathbb{E}\left[(X(t))^{2}\right]-(\mathbb{E}[X(t)])^{2} \\
& =\frac{-\mathrm{e}^{2 / t}\left(\int_{1 / t}^{\infty} \mathrm{e}^{-\xi} / \xi \mathrm{d} \xi\right)^{2}-\mathrm{e}^{1 / t} \int_{1 / t}^{\infty} \mathrm{e}^{-\xi} / \xi \mathrm{d} \xi+t}{t^{4}} .
\end{aligned}
$$

Plots for the mean and the variance are shown in Figure 3.2. Notice that these plots are in agreement with the plot of the $1-P D F f_{1}(x, t)$. Indeed, as the mean tends to stabilize as $t$ increases, hence the variance goes to zero and the shape of $f_{1}(x, t)$ becomes leptokurtic.

In order to invoke the intuition that connects random results with their deterministic counterpart, it is interesting to notice that if that averaged IVP associated with this example is considered,

$$
\left.\begin{array}{l}
\frac{\mathrm{d}}{\mathrm{d} t}(\mathbb{E}[X(t)])=-(\mathbb{E}[X(t)])^{2}, \\
\mathbb{E}[X(0)]=1,
\end{array}\right\}
$$

its solution is $\mathbb{E}[X(t)]=1 /(1+t)$, which converges to 0 as $t$ tends to $+\infty$. This is in accordance with the behaviour of the 1-PDF shown in Figure 3.1 where one observes that the mass of probability concentrates about $x=0$ as $t$ increases.

\section{Case II.2: $\mathrm{D}$ is a random variable}

Let us assume that non-linear coefficient $D$ is a RV with $\operatorname{PDF} f_{D}(d)$ and initial condition is a deterministic constant $x_{0}$. In agreement with Table 3.1 and identification (3.4), it corresponds to $\operatorname{IVP}(3.2)$

$$
\left.\begin{array}{l}
\dot{Z}(t)=B, \\
Z(0)=z_{0},
\end{array}\right\} \quad z_{0}=\frac{1}{x_{0}}, \quad B=-D
$$

For $t>0$ fixed, according to [16, Equation (64)] the PDF of solution SP of IVP (3.10) evaluated at that $t$ is given by 


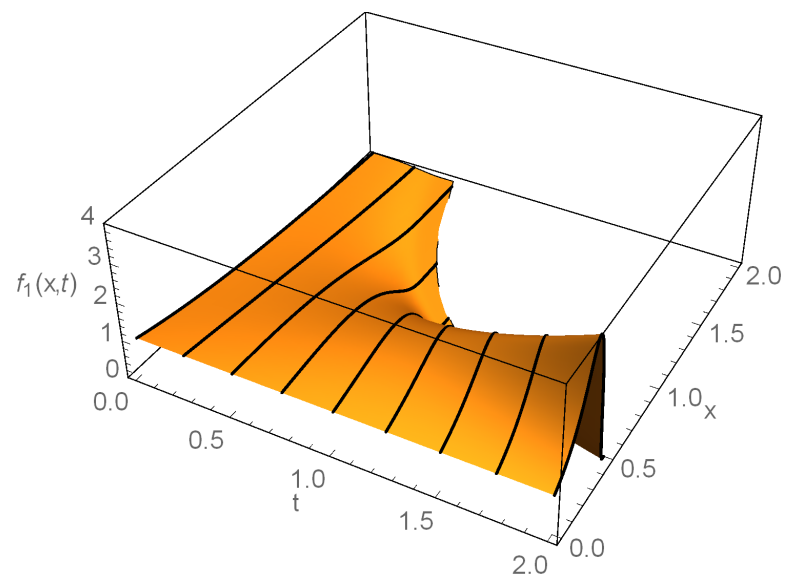

Figure 3.1: Plot of the 1-PDF $f_{1}(x, t)$ given by (3.9) in Example 3.1 at different values of $t \in\{0,0.25,0.5,0.75, \ldots, 2\}$ (corresponding to the solid lines) with $X_{0} \sim \operatorname{Exp}(\lambda=1)$ and $d=-1$.

$$
f_{Z}(z)=\frac{1}{t} f_{B}\left(\frac{z-z_{0}}{t}\right)
$$

Next, the above expression is represented in terms of the PDF of RV $D$ taking into account (3.10) and Proposition 2.6 to $U=D, V=B, a=-1$ and $b=0$

$$
f_{Z}(z)=\frac{1}{t} f_{B}\left(\frac{z-1 / x_{0}}{t}\right)=\frac{1}{t} f_{D}\left(\frac{1-z x_{0}}{x_{0} t}\right) .
$$

Finally, as $X(t)=1 / Z(t)$, applying (3.11) and Proposition 2.2 to $U=Z$ and $V=X$, with $Z=Z(t)$ and $X=X(t)$, for each $t>0$,

$$
f_{X}(x)=\frac{1}{x^{2}} f_{Z}\left(\frac{1}{x}\right)=\frac{1}{x^{2}} \frac{1}{t} f_{D}\left(\frac{1-\frac{1}{x} x_{0}}{x_{0} t}\right)=\frac{1}{x^{2} t} f_{D}\left(\frac{x-x_{0}}{x x_{0} t}\right)
$$

Therefore, in this case the 1-PDF of solution SP $X(t)$ of IVP (3.1) is given by

$$
f_{1}(x, t)=\frac{1}{x^{2} t} f_{D}\left(\frac{x-x_{0}}{x x_{0} t}\right), \quad t>0 .
$$



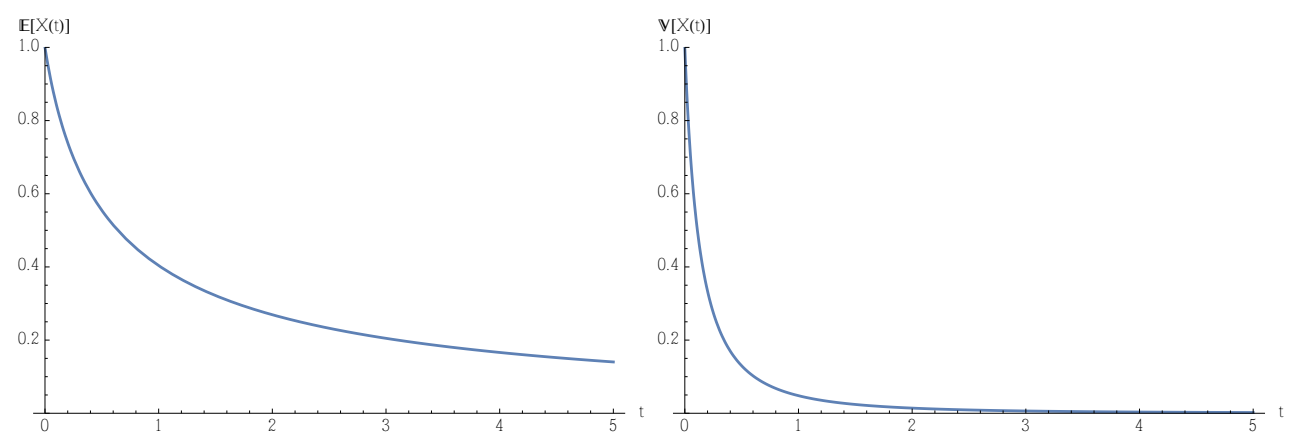

Figure 3.2: Plot of the expectation (left) and the variance (right) of solution SP in Example 3.1 with $0 \leq t \leq 5$.

If $t=0, X(0)=x_{0}$ and then

$$
f_{1}(x, 0)=\delta\left(x-x_{0}\right), \quad-\infty<x<\infty
$$

where $\delta(\cdot)$ denotes the Dirac delta function.

Example 3.2 Let us take $x_{0}=1$ and $D$ a standard Gaussian $R V, D \sim \mathrm{N}(0 ; 1)$. According to (3.12) the 1-PDF to solution SP X(t) of IVP (3.1) is given by

$$
f_{1}(x, t)=\frac{\mathrm{e}^{-\frac{(x-1)^{2}}{2 t^{2} x^{2}}}}{\sqrt{2 \pi} t x^{2}} .
$$

In Figure 3.3 a plot of $f_{1}(x, t)$ is shown. One observes that the variability of the solution decreases as $t$ goes on and the 1-PDF concentrates about $x=0$.

\section{Case II.3: $\left(\mathrm{X}_{0}, \mathrm{D}\right)$ is a random vector}

In this context, it is assumed that both, initial condition $X_{0}$, and non-linear coefficient $D$, are RVs with joint PDF $f_{X_{0}, D}\left(x_{0}, d\right)$. As it is listed in Table 3.1 and considering identification (3.4), this case corresponds to the following specialization of IVP (3.2)

$$
\left.\begin{array}{l}
\dot{Z}(t)=B \\
Z(0)=Z_{0},
\end{array}\right\} \quad Z_{0}=\frac{1}{X_{0}}, \quad B=-D
$$




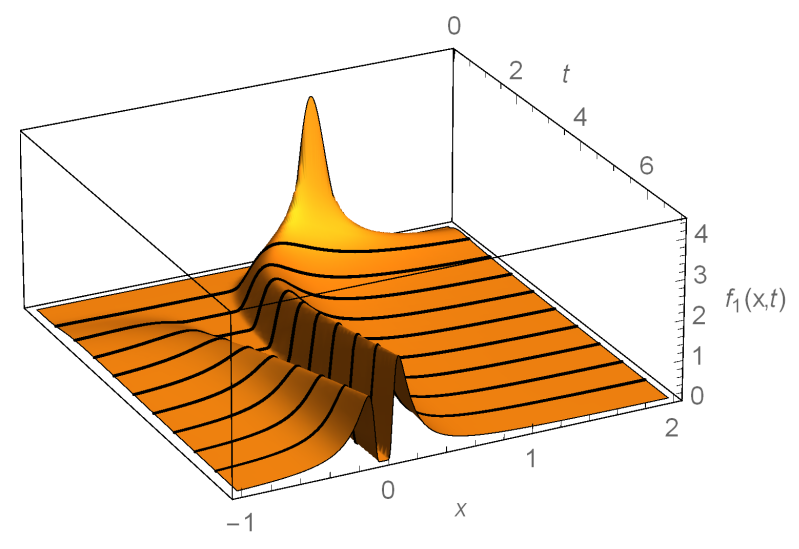

Figure 3.3: Plot of the 1-PDF $f_{1}(x, t)$ given by (3.13) in Example 3.2 at different values of $t \in\{0,0.25,0.5,0.75, \ldots, 2\}$ (corresponding to the solid lines) with $x_{0}=1$ and $D \sim \mathrm{N}(0 ; 1)$.

Let us fix $t>0$, according to [16, Equation (74)] the PDF of solution SP of IVP (3.14) evaluated at that $t$ is given by

$$
f_{Z}(z)=\frac{1}{t} \int_{\mathcal{D}\left(Z_{0}\right)} f_{Z_{0}, B}\left(\xi, \frac{z-\xi}{t}\right) \mathrm{d} \xi
$$

where $\mathcal{D}\left(Z_{0}\right)$ denotes the domain of RV $Z_{0}$. Now, applying Proposition 2.3 to $U_{1}=X_{0}, U_{2}=D, V_{1}=Z_{0}$ and $V_{2}=B,(3.15)$ can be expressed in terms of the joint $\mathrm{PDF} f_{X_{0}, D}\left(x_{0}, d\right)$

$$
f_{Z}(z)=\frac{1}{t} \int_{\mathcal{D}\left(1 / X_{0}\right)} \frac{1}{\xi^{2}} f_{X_{0}, D}\left(\frac{1}{\xi}, \frac{\xi-z}{t}\right) \mathrm{d} \xi
$$

For each $t>0$, by (3.3) $X=1 / Z$ and, applying Proposition 2.2 one gets

$$
f_{X}(x)=\frac{1}{x^{2}} f_{Z}\left(\frac{1}{x}\right)=\frac{1}{x^{2} t} \int_{\mathcal{D}\left(1 / X_{0}\right)} \frac{1}{\xi^{2}} f_{X_{0}, D}\left(\frac{1}{\xi}, \frac{x \xi-1}{t x}\right) \mathrm{d} \xi
$$

Therefore, in this case the 1-PDF of solution SP $X(t)$ of IVP (3.1) is given by

$$
f_{1}(x, t)=\frac{1}{x^{2} t} \int_{\mathcal{D}\left(1 / X_{0}\right)} \frac{1}{\xi^{2}} f_{X_{0}, D}\left(\frac{1}{\xi}, \frac{x \xi-1}{t x}\right) \mathrm{d} \xi, \quad t>0 .
$$


In accordance with $(2.18)$, the domain $\mathcal{D}\left(1 / X_{0}\right)$ can be easily computed from $\mathcal{D}\left(X_{0}\right)$, which is assumed to be known.

If $t=0$, as $X(0)=X_{0}$ the 1 -PDF is just the marginal PDF of the joint PDF $f_{X_{0}, D}\left(x_{0}, d\right)$, hence

$$
f_{1}(x, 0)=\int_{\mathcal{D}(D)} f_{X_{0}, D}\left(x_{0}, d\right) \mathrm{d} d
$$

Example 3.3 Let us assume that the joint PDF of random vector $\left(X_{0}, D\right)$ is given by

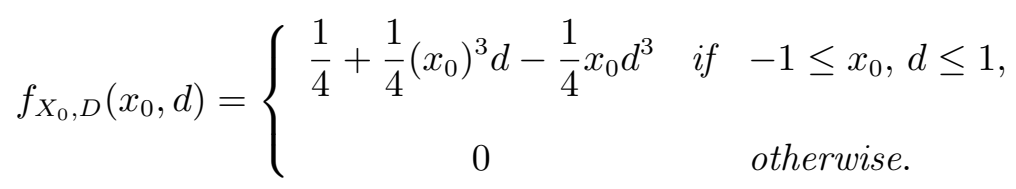

A plot of $f_{1}(x, t)$ is depicted in Figure 3.4. From it, one sees that for each the probability of solution $S P X(t)$ distributes symmetrically about $x=0$ becoming leptokurtic as $t$ increases.

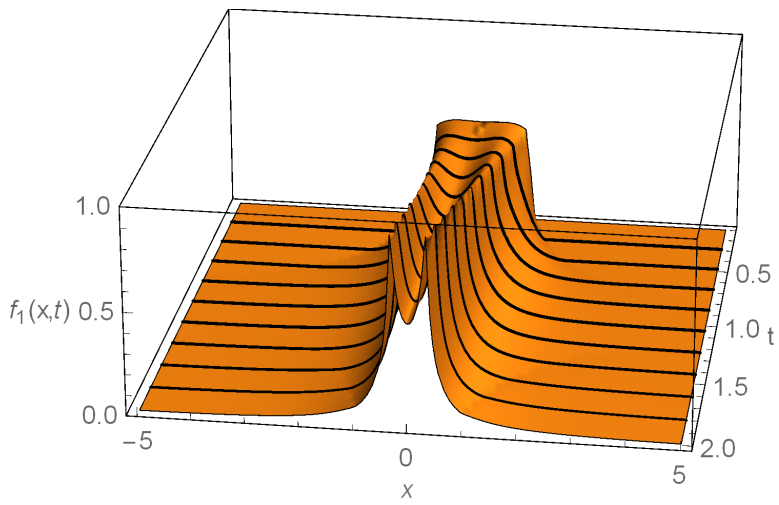

Figure 3.4: Plot of the 1-PDF $f_{1}(x, t)$ in Example 3.3 at different values of $t \in\{0,0.25,0.5$, $0.75, \ldots, 2\}$ (corresponding to the solid lines) in the case that $\left(X_{0}, D\right)$ has the joint PDF given by (3.16). 


\subsubsection{Solving the Cases III.1-III.7}

This subsection is devoted to provide explicit formulas for the 1-PDF, $f_{1}(x, t)$, of solution SP of IVP (3.1) in each one of the Cases III.1-III.7 listed in Table 3.1. Notice that in contrast to what was assumed when analysing Cases II.1II.3, throughout this subsection linear coefficient $C$ can be either deterministic and different from zero, or a RV. As it was indicated previously, in the first case it will be denoted by $c$ and in the latter as $C$.

\section{Case III.1: $\mathrm{X}_{0}$ is a random variable}

Let $f_{X_{0}}\left(x_{0}\right)$ be the PDF of RV $X_{0}, c \in \mathbb{R}-\{0\}$ and $d \in \mathbb{R}$. According to Table 3.1 and (3.4), it corresponds to the following particular case of IVP (3.2)

$$
\left.\begin{array}{l}
\dot{Z}(t)=a Z(t)+b, \\
Z(0)=Z_{0},
\end{array}\right\} \quad Z_{0}=\frac{1}{X_{0}}, \quad b=-d, \quad a=-c .
$$

Let us fix $t \geq 0$, then by [16, Equation (84)] the PDF of solution SP of IVP (3.17) evaluated at that $t$ is given by

$$
f_{Z}(z)=\mathrm{e}^{-a t} f_{Z_{0}}\left(\mathrm{e}^{-a t}\left(z+\frac{b}{a}\right)-\frac{b}{a}\right) .
$$

Now, taking into account (3.17) and Proposition 2.2 to $U=X_{0}, V=Z_{0}, f_{Z}(z)$ can be expressed as follows

$$
\begin{aligned}
f_{Z}(z) & =\frac{\mathrm{e}^{c t}}{\left(\mathrm{e}^{c t}\left(z+\frac{d}{c}\right)-\frac{d}{c}\right)^{2}} f_{X_{0}}\left(\frac{1}{\mathrm{e}^{c t}\left(z+\frac{d}{c}\right)-\frac{d}{c}}\right) \\
& =\frac{c^{2} \mathrm{e}^{c t}}{\left(\mathrm{e}^{c t}(z c+d)-d\right)^{2}} f_{X_{0}}\left(\frac{c}{\mathrm{e}^{c t}(z c+d)-d}\right) .
\end{aligned}
$$

Following the same argument exhibited in the previous cases, for each $t \geq 0$, this PDF can be expressed as a function of RV $X=1 / Z$ by applying Proposition 2.2 , this yields

$$
f_{X}(x)=\frac{1}{x^{2}} f_{Z}\left(\frac{1}{x}\right)=\frac{c^{2} \mathrm{e}^{c t}}{\left(\mathrm{e}^{c t}(c+d x)-d x\right)^{2}} f_{X_{0}}\left(\frac{c x}{\mathrm{e}^{c t}(c+d x)-d x}\right) .
$$


Taking $t \geq 0$ arbitrary, the 1-PDF of solution SP $X(t)$ of IVP (3.1) is given by

$$
f_{1}(x, t)=\frac{c^{2} \mathrm{e}^{c t}}{\left(\mathrm{e}^{c t}(c+d x)-d x\right)^{2}} f_{X_{0}}\left(\frac{c x}{\mathrm{e}^{c t}(c+d x)-d x}\right), \quad t \geq 0 .
$$

Example 3.4 Let $c=1 / 2, d=-1$ and $X_{0}$ a standard Gaussian $R V, X_{0} \sim$ $\mathrm{N}(0 ; 1)$ be the input parameters of IVP (3.1). According to (3.18), the 1-PDF to solution $S P X(t)$ to this IVP is given by

$$
f_{1}(x, t)=\frac{\mathrm{e}^{\left.\frac{t}{2}-\frac{x^{2}}{8\left(\mathrm{e}^{t / 2}\left(\frac{1}{2}-x\right)+x\right.}\right)^{2}}}{4 \sqrt{2 \pi}\left(\mathrm{e}^{t / 2}\left(\frac{1}{2}-x\right)+x\right)^{2}} .
$$

Figure 3.5 shows the plot of $f_{1}(x, t)$. From this representation, one observes that the variability of the 1-PDF reduces as $t$ increases.

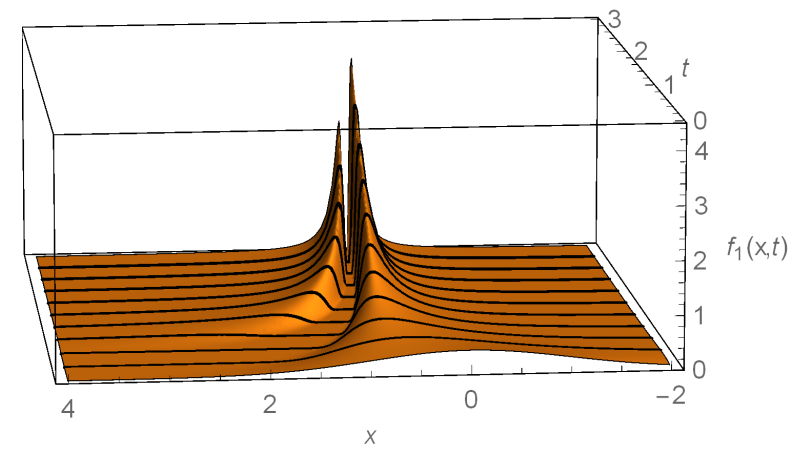

Figure 3.5: Plot of the 1-PDF $f_{1}(x, t)$ given by (3.19) in Example 3.4 at different values of $t \in\{0,0.25,0.5,0.75, \ldots, 2\}$ (corresponding to the solid lines) with $c=1 / 2, d=-1$ and $X_{0} \sim \mathrm{N}(0 ; 1)$.

\section{Case III.2: $\mathrm{D}$ is a random variable}

Let $f_{D}(d)$ be the PDF of RV $D$ and let us assume that both initial condition $x_{0}$ and linear coefficient $c$ are deterministic constants. Taking into account Table 
3.1 and (3.4), this case corresponds to the following particularization of IVP $(3.2)$

$$
\left.\begin{array}{l}
\dot{Z}(t)=a Z(t)+B, \\
Z(0)=z_{0},
\end{array}\right\} \quad z_{0}=\frac{1}{x_{0}}, \quad B=-D, \quad a=-c .
$$

For each $t>0$ fixed, by applying [16, Equation (92)] the following expression for the PDF of solution SP of IVP (3.20) evaluated at that $t$ is obtained

$$
f_{Z}(z)=\frac{a}{\mathrm{e}^{a t}-1} f_{B}\left(\frac{a\left(z-z_{0} \mathrm{e}^{a t}\right)}{\mathrm{e}^{a t}-1}\right) .
$$

This PDF can be expressed in terms of data $x_{0}, D$ and $c$ by considering (3.20), and applying Proposition 2.6 to $U=D, V=B, a=-1$ and $b=0$ this yields

$$
f_{Z}(z)=\frac{c}{1-\mathrm{e}^{-c t}} f_{D}\left(\frac{-c\left(1-z x_{0} \mathrm{e}^{c t}\right)}{x_{0}\left(1-\mathrm{e}^{c t}\right)}\right) .
$$

Finally, taking into account that $X=1 / Z$ and applying Proposition 2.2, the 1-PDF of solution SP $X(t)$ of IVP (3.1) is obtained as follows

$$
f_{1}(x, t)=\frac{c}{x^{2}\left(1-\mathrm{e}^{-c t}\right)} f_{D}\left(\frac{-c\left(x-x_{0} \mathrm{e}^{c t}\right)}{x x_{0}\left(1-\mathrm{e}^{c t}\right)}\right), \quad t>0 .
$$

For $t=0$, as $X(0)=x_{0}$ one gets

$$
f_{1}(x, 0)=\delta\left(x-x_{0}\right), \quad-\infty<x<\infty .
$$

Example 3.5 Let us consider IVP (3.1) with $x_{0}=1, c=-1$ and $X_{0}$ a Gamma $R V$ of parameters $\alpha=4$ and $\beta=2, X_{0} \sim \mathrm{Ga}(4 ; 2)$. In Figure 3.5, the 1-PDF of solution SP given by (3.21) is plotted. One observes that the probability density concentrates about $x=0$ as $t$ increases. 


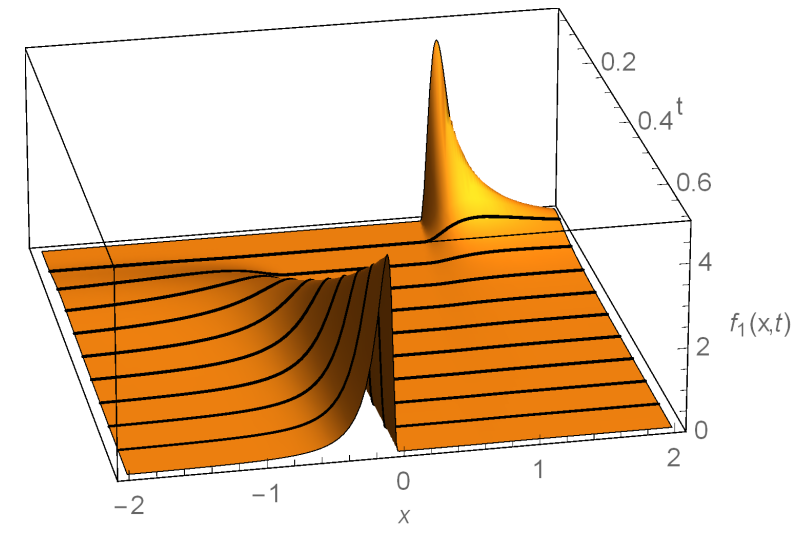

Figure 3.6: Plot of the 1-PDF $f_{1}(x, t)$ given by (3.21) in Example 3.5 at different values of $t \in\{0,0.25,0.5,0.75, \ldots, 2\}$ (corresponding to the solid lines) with $x_{0}=1, c=-1$ and $D \sim \mathrm{Ga}(4 ; 2)$.

\section{Case III.3: $\mathrm{C}$ is a random variable}

So far the computation of the 1-PDF of solution SP of non-linear IVP (3.1) has relied on the application of the results previously established for linear IVP (3.2) in [16]. In fact, notice that explicit expressions of the 1-PDF of solution of IVP (3.2) in each one of the Cases II.1-II.3 and Cases III.1-III.2 have been used to obtain a closed expression of the 1-PDF in the corresponding cases to IVP (3.1). Unfortunately, this strategy is not feasible when the single random input in (3.1) is the coefficient $C$ since the complexity of the approximate expression to the 1-PDF of the underlying linear IVP (3.2) (see Case III.3 of [16]). To overcome this drawback, the same strategy used in the Case III.3 of [16] will be applied, but directly on the closed expression of solution SP of the non-linear IVP (3.1), which in the current case is given by

$$
X(t)=\frac{C x_{0} \mathrm{e}^{C t}}{C+d x_{0}-d x_{0} \mathrm{e}^{C t}} .
$$

In order to apply RVT technique, for each $t \geq 0$, first from (3.22) the mapping $r(c)=\left(c x_{0} \mathrm{e}^{c t}\right) /\left(c+d x_{0}-d x_{0} \mathrm{e}^{c t}\right)$ is defined. As it is not possible to isolate $c$ to determine the inverse mapping, say $s$ of $r, s$ is approximated using the Lagrange-Bürmann theorem which permits to calculate the inverse mapping of an analytic function. This approximation comes from the truncation of 
an infinite series (see [16, Theorem 19]). As can be checked in detail in the analysis of the Case III.1 studied in [16], the 1-PDF of solution SP (3.22) can be represented as follows

$$
f_{1}(x, t)=\sum_{j=1}^{k} f_{C}\left(s_{j, N_{j}}\right)\left|\frac{\mathrm{d} s_{j, N_{j}}(x)}{\mathrm{d} x}\right|,
$$

where $f_{C}(c)$ represents the PDF of RV $C, k$ denotes the number of subintervals in which the domain of RV $C$ must be split to guarantee that the mapping $r$ is monotone and $s_{j, N_{j}}$ is the approximation of the inverse mapping $s$ on the subinterval $j$, using truncation of order $N_{j}, 1 \leq j \leq k$.

Example 3.6 Let us assume that $x_{0}=1, d=1$ and $C \sim \operatorname{Be}(\alpha=2 ; \beta=3)$. In Figure 3.7, the 1-PDF of solution SP of IVP (3.1), using Lagrange-Bürmann theorem and (3.23) for different values of t, has been plotted. To carry out these computations the domain of $R V C$ has been split into $k=1$ piece. One observes that the variance of solution $S P X(t)$ increases as $t$ goes on.

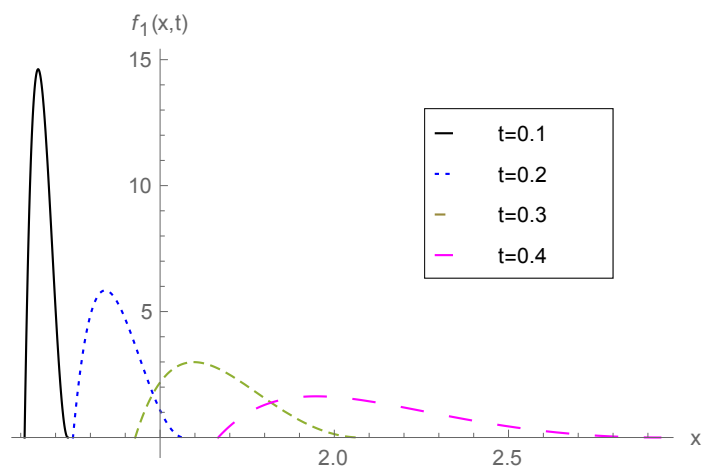

Figure 3.7: Plot of the 1-PDF $f_{1}(x, t)$ in Example 3.6 at different values of $t \in\{0.1,0.2$, $0.3,0.4\}$ with $x_{0}=1, d=1$ and $C \sim \operatorname{Be}(\alpha=2 ; \beta=3)$. 


\section{Case III.4: $\left(\mathrm{X}_{0}, \mathrm{D}\right)$ is a random vector}

Now, initial condition $X_{0}$ and non-linear coefficient $D$ are assumed to be RVs whose joint PDF is denoted by $f_{X_{0}, D}\left(x_{0}, d\right)$, while parameter $c$ is deterministic. In agreement to Table 3.1 and (3.4), this corresponds to the following particular case of IVP (3.2)

$$
\left.\begin{array}{l}
\dot{Z}(t)=a Z(t)+B, \\
Z(0)=Z_{0}
\end{array}\right\} \quad Z_{0}=\frac{1}{X_{0}}, \quad B=-D, \quad a=-c
$$

Let $t>0$ be fixed, then applying [16, Equation (110)], the PDF of solution SP of IVP (3.24) evaluated at that $t$ is obtained

$$
\begin{aligned}
f_{Z}(z) & =\int_{\mathcal{D}\left(Z_{1}\right)} f_{Z_{0}, B}\left(\xi \mathrm{e}^{-a t}, \frac{a(z-\xi)}{\mathrm{e}^{a t}-1}\right) \frac{a \mathrm{e}^{-a t}}{\mathrm{e}^{a t}-1} \mathrm{~d} \xi \\
& =\int_{\mathcal{D}\left(Z_{1}\right)} f_{Z_{0}, B}\left(\xi \mathrm{e}^{c t}, \frac{-c(z-\xi)}{\mathrm{e}^{-c t}-1}\right) \frac{c \mathrm{e}^{c t}}{1-\mathrm{e}^{-c t}} \mathrm{~d} \xi
\end{aligned}
$$

where $Z_{1}=\mathrm{e}^{a t} Z_{0}$. The PDF $f_{Z}(z)$ can be represented in terms of $\left(X_{0}, D\right)$, taking into account (3.24) and applying Proposition 2.3 to $U_{1}=X_{0}, U_{2}=D$, $V_{1}=Z_{0}$ and $V_{2}=B$

$$
f_{Z}(z)=\frac{c}{\mathrm{e}^{c t}-1} \int_{\mathcal{D}\left(Z_{1}\right)} f_{X_{0}, D}\left(\frac{1}{\xi \mathrm{e}^{c t}}, \frac{c(z-\xi)}{\mathrm{e}^{-c t}-1}\right) \frac{1}{\xi^{2}} \mathrm{~d} \xi
$$

By (3.3), $X(t)=1 / Z(t)$ for each $t>0$, then denoting $X=X(t)$ and $Z=Z(t)$ the application of Proposition 2.2 yields

$$
f_{X}(x)=\frac{1}{x^{2}} f_{Z}\left(\frac{1}{x}\right)=\frac{1}{x^{2}} \frac{c}{\mathrm{e}^{c t}-1} \int_{\mathcal{D}\left(Z_{1}\right)} f_{X_{0}, D}\left(\frac{1}{\xi \mathrm{e}^{c t}}, \frac{c(1-\xi x)}{x\left(\mathrm{e}^{-c t}-1\right)}\right) \frac{1}{\xi^{2}} \mathrm{~d} \xi .
$$

Finally, taking into account that $Z_{1}=\mathrm{e}^{a t} Z_{0}=1 /\left(\mathrm{e}^{c t} X_{0}\right)$, the $1-\mathrm{PDF}$ of solution SP $X(t)$ of IVP (3.1) is given by

$$
f_{1}(x, t)=\frac{c}{x^{2}\left(\mathrm{e}^{c t}-1\right)} \int_{\mathcal{D}\left(\frac{1}{\mathrm{e}^{c t} X_{0}}\right)} f_{X_{0}, D}\left(\frac{1}{\xi \mathrm{e}^{c t}}, \frac{c(1-\xi x)}{x\left(\mathrm{e}^{-c t}-1\right)}\right) \frac{1}{\xi^{2}} \mathrm{~d} \xi, \quad t>0 .
$$


If $t=0$, as $X(0)=X_{0}$, the 1 -PDF of $X(t)$ is the $D$-marginal PDF of $f_{X_{0}, D}\left(x_{0}, d\right)$

$$
f_{1}(x, 0)=\int_{\mathcal{D}(D)} f_{X_{0}, D}\left(x_{0}, d\right) \mathrm{d} d
$$

Example 3.7 Let us assume that $c=-1$ and the joint PDF of random vector $\left(X_{0}, D\right)$ is a bivariate Gaussian distribution with mean vector $\boldsymbol{\mu}$ and variancecovariance matrix $\boldsymbol{\Sigma}$ given by

$$
\begin{aligned}
& \boldsymbol{\mu}=\left(\mu_{X_{0}}, \mu_{D}\right)=(1,0), \quad \boldsymbol{\Sigma}=\left(\begin{array}{cc}
\sigma_{X_{0}}^{2} & \rho_{X_{0}, D} \sigma_{X_{0}} \sigma_{D} \\
\rho_{X_{0}, D} \sigma_{X_{0}} \sigma_{D} & \sigma_{D}^{2}
\end{array}\right), \\
& \sigma_{X_{0}}=\sigma_{D}=1 / 10, \rho_{X_{0}, D}=1 / 2 .
\end{aligned}
$$

Figure 3.8 shows a piece of surface which defines the 1-PDF As in previous cases, $f_{1}(x, t)$ has less variability as $t$ increases.

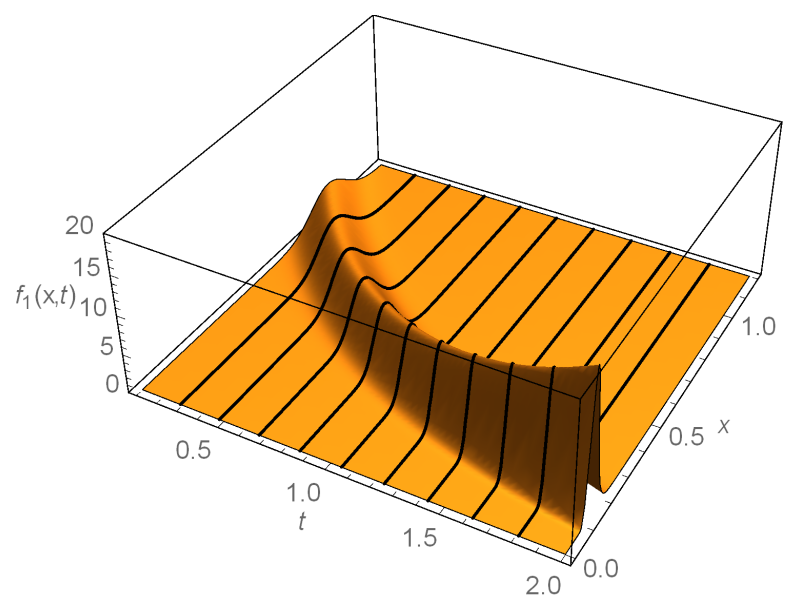

Figure 3.8: Plot of the 1-PDF $f_{1}(x, t)$ in Example 3.7 at different values of $t \in\{0,0.25,0.5$, $0.75, \ldots, 2\}$ (corresponding to the solid lines) in the case that $c=-1$ and $X_{0}$ and $D$ are correlated RVs according to a bivariate Gaussian distribution with mean vector $\boldsymbol{\mu}$ and variancecovariance matrix $\boldsymbol{\Sigma}$ given by (3.25). 


\section{Case III.5: $\left(\mathrm{X}_{0}, \mathrm{C}\right)$ is a random vector}

Let us denote by $f_{X_{0}, C}\left(x_{0}, c\right)$ the joint PDF of random vector $\left(X_{0}, C\right)$ and let us assume that parameter $d$ is a deterministic constant. In this context according to Table 3.1 and (3.4), IVP (3.2) is written as

$$
\left.\begin{array}{l}
\dot{Z}(t)=A Z(t)+b, \\
Z(0)=Z_{0},
\end{array}\right\} \quad Z_{0}=\frac{1}{X_{0}}, \quad b=-d, \quad A=-C .
$$

Let us fix $t \geq 0$, then applying [16, Equation (126)] the PDF of solution SP of IVP (3.26) evaluated at that $t$ can be written as

$$
\begin{aligned}
f_{Z}(z) & =\int_{\mathcal{D}\left(Z_{2}\right)} \frac{|b|}{\xi^{2}} \mathrm{e}^{\frac{b}{\xi} t} f_{Z_{0}, A}\left(z \mathrm{e}^{\frac{b}{\xi} t}+\xi\left(1-\mathrm{e}^{\frac{b}{\xi} t}\right), \frac{-b}{\xi}\right) \mathrm{d} \xi \\
& =\int_{\mathcal{D}\left(Z_{2}\right)} \frac{|d|}{\xi^{2}} \mathrm{e}^{-\frac{d}{\xi} t} f_{Z_{0}, A}\left(z \mathrm{e}^{-\frac{d}{\xi} t}+\xi\left(1-\mathrm{e}^{-\frac{d}{\xi} t}\right), \frac{d}{\xi}\right) \mathrm{d} \xi
\end{aligned}
$$

where $Z_{2}=-b / A$. $f_{Z}(z)$ can be represented in terms of $\left(X_{0}, C\right)$ by applying Proposition 2.3 to $U_{1}=X_{0}, U_{2}=C, V_{1}=Z_{0}$ and $V_{2}=A$ as follows

$$
\begin{aligned}
f_{Z}(z)= & \int_{\mathcal{D}\left(Z_{2}\right)} \frac{|d|}{\xi^{2}} \mathrm{e}^{-\frac{d}{\xi} t} f_{X_{0}, C}\left(\frac{1}{z \mathrm{e}^{-\frac{d}{\xi} t}+\xi\left(1-\mathrm{e}^{-\frac{d}{\xi} t}\right)},-\frac{d}{\xi}\right) \\
& \times \frac{1}{\left(z \mathrm{e}^{-\frac{d}{\xi} t}+\xi\left(1-\mathrm{e}^{-\frac{d}{\xi} t}\right)\right)^{2}} \mathrm{~d} \xi
\end{aligned}
$$

Taking into account that $X(t)=1 / Z(t)$ for each $t \geq 0, f_{Z}(z)$ can be represented in terms of $X$ applying Proposition 2.2 


$$
\begin{aligned}
f_{X}(x)= & \frac{1}{x^{2}} f_{Z}\left(\frac{1}{x}\right) \\
= & \int_{\mathcal{D}\left(Z_{2}\right)} \frac{|d|}{\xi^{2}} \mathrm{e}^{-\frac{d}{\xi} t} f_{X_{0}, C}\left(\frac{x}{\mathrm{e}^{-\frac{d}{\xi} t}+\xi x\left(1-\mathrm{e}^{-\frac{d}{\xi} t}\right)},-\frac{d}{\xi}\right) \\
& \times \frac{1}{\left(\mathrm{e}^{-\frac{d}{\xi} t}+\xi x\left(1-\mathrm{e}^{-\frac{d}{\xi} t}\right)\right)^{2}} \mathrm{~d} \xi
\end{aligned}
$$

As $Z_{2}=-b / A=-d / C$, the domain of the above integral can be expressed in terms of data. Hence, the 1-PDF of solution SP $X(t)$ of IVP (3.1) is given by

$$
\begin{aligned}
f_{1}(x, t)= & \int_{\mathcal{D}(-d / C)} \frac{|d|}{\xi^{2}} \mathrm{e}^{-\frac{d}{\xi} t} f_{X_{0}, C}\left(\frac{x}{\mathrm{e}^{-\frac{d}{\xi} t}+\xi x\left(1-\mathrm{e}^{-\frac{d}{\xi} t}\right)},-\frac{d}{\xi}\right) \\
& \times \frac{1}{\left(\mathrm{e}^{-\frac{d}{\xi} t}+\xi x\left(1-\mathrm{e}^{-\frac{d}{\xi} t}\right)\right)^{2}} \mathrm{~d} \xi .
\end{aligned}
$$

Example 3.8 Let us take $d=1$ and $\left(X_{0}, C\right) \sim \mathrm{N}(\boldsymbol{\mu} ; \boldsymbol{\Sigma})$, where $\boldsymbol{\mu}$ and $\boldsymbol{\Sigma}$ are defined by (3.25). In Figure 3.9 a plot of the $1-P D F f_{1}(x, t)$ given by (3.27) is shown. From it, one observes that the variance of solution SP of the corresponding IVP (3.1) increases as $t$ does.

\section{Case III.6: $(\mathrm{D}, \mathrm{C})$ is a random vector}

Throughout this part, $f_{D, C}(d, c)$ will denote the PDF of random vector $(D, C)$ and initial condition will be assumed to be a deterministic constant $x_{0}$. Notice that, in accordance with Table 3.1 and (3.4), this case corresponds with the following specialization of IVP (3.2)

$$
\left.\begin{array}{l}
\dot{Z}(t)=A Z(t)+B, \\
Z(0)=z_{0},
\end{array}\right\} \quad z_{0}=\frac{1}{x_{0}}, \quad B=-D, \quad A=-C .
$$

Let $t>0$ be fixed, applying [16, Equation (140)] the PDF of solution SP of IVP (3.28) evaluated at that $t$ is given by 


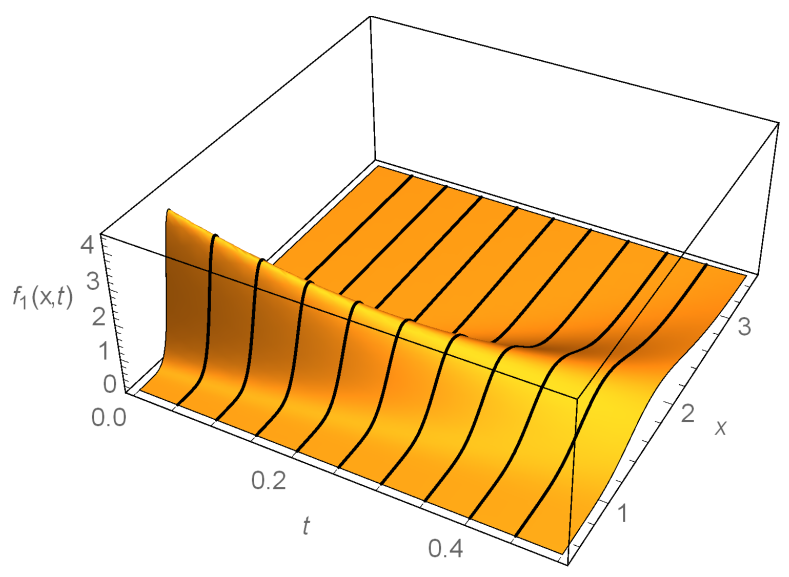

Figure 3.9: Plot of the 1-PDF $f_{1}(x, t)$ in Example 3.8 at different values of $t \in\{0,0.25,0.5$, $0.75, \ldots, 2\}$ (corresponding to the solid lines) in the case that $d=1$ and $X_{0}$ and $C$ are correlated RVs according to a bivariate Gaussian distribution with mean vector $\boldsymbol{\mu}$ and variancecovariance matrix $\boldsymbol{\Sigma}$ given by (3.25).

$$
\begin{aligned}
f_{Z}(z)= & \frac{z_{0}}{t^{2}} \int_{\mathcal{D}\left(Z_{2}\right)} f_{B, A}\left(\frac{z_{0}(z-\xi)}{t} \frac{\log (\xi)-\log \left(z_{0}\right)}{\xi-z_{0}}, \frac{\log (\xi)-\log \left(z_{0}\right)}{t}\right) \\
& \times \frac{1}{\xi}\left|\frac{\log (\xi)-\log \left(z_{0}\right)}{\xi-z_{0}}\right| \mathrm{d} \xi \\
= & \frac{1}{x_{0} t^{2}} \int_{\mathcal{D}\left(Z_{2}\right)} f_{B, A}\left(\frac{z-\xi}{t} \frac{\log (\xi)+\log \left(x_{0}\right)}{\xi x_{0}-1}, \frac{\log (\xi)+\log \left(x_{0}\right)}{t}\right) \\
& \times \frac{\left|x_{0}\right|}{\xi}\left|\frac{\log (\xi)+\log \left(x_{0}\right)}{\xi x_{0}-1}\right| \mathrm{d} \xi
\end{aligned}
$$

where $Z_{2}=z_{0} \mathrm{e}^{A t}$. The PDF $f_{Z}(t)$ can be expressed in terms of random vector $(D, C)$ applying Proposition 2.4 to $U_{1}=D, U_{2}=C, V_{1}=B$ and $V_{2}=A$,

$$
\begin{aligned}
f_{Z}(z)= & \frac{1}{x_{0} t^{2}} \int_{\mathcal{D}\left(Z_{2}\right)} f_{D, C}\left(\frac{z-\xi}{t} \frac{\log (\xi)+\log \left(x_{0}\right)}{1-\xi x_{0}},-\frac{\log (\xi)+\log \left(x_{0}\right)}{t}\right) \\
& \times \frac{\left|x_{0}\right|}{\xi}\left|\frac{\log (\xi)+\log \left(x_{0}\right)}{\xi x_{0}-1}\right| \mathrm{d} \xi
\end{aligned}
$$


Now, applying Proposition 2.2 to $X=1 / Z, f_{Z}(z)$ is represented in terms of $X$

$$
\begin{aligned}
f_{X}(x)= & \frac{1}{x^{2}} f_{Z}\left(\frac{1}{x}\right) \\
= & \frac{1}{x^{2}} \frac{1}{x_{0} t^{2}} \int_{\mathcal{D}\left(Z_{2}\right)} f_{D, C}\left(\frac{1-\xi x}{x t} \frac{\log (\xi)+\log \left(x_{0}\right)}{1-\xi x_{0}},-\frac{\log (\xi)+\log \left(x_{0}\right)}{t}\right) \\
& \times \frac{\left|x_{0}\right|}{\xi}\left|\frac{\log (\xi)+\log \left(x_{0}\right)}{\xi x_{0}-1}\right| \mathrm{d} \xi .
\end{aligned}
$$

As $Z_{2}=z_{0} \mathrm{e}^{A t}=1 /\left(x_{0} \mathrm{e}^{C t}\right)$, the 1-PDF of solution SP $X(t)$ to IVP (3.1) is given by

$$
\begin{aligned}
& f_{1}(x, t) \\
& =\frac{1}{x^{2} x_{0} t^{2}} \int_{\mathcal{D}\left(1 /\left(x_{0} \mathrm{e}^{\mathrm{C}}\right)\right)} f_{D, C}\left(\frac{1-\xi x}{x t} \frac{\log (\xi)+\log \left(x_{0}\right)}{1-\xi x_{0}},-\frac{\log (\xi)+\log \left(x_{0}\right)}{t}\right) \\
& \quad \times \frac{\left|x_{0}\right|}{\xi}\left|\frac{\log (\xi)+\log \left(x_{0}\right)}{\xi x_{0}-1}\right| \mathrm{d} \xi .
\end{aligned}
$$

If $t=0$, as $X(0)=x_{0}$ one gets

$$
f_{1}(x, 0)=\delta\left(x-x_{0}\right), \quad-\infty<x<\infty .
$$

Example 3.9 Let us assume that $x_{0}=1$ and the joint PDF of random vector $(D, C)$ is given by

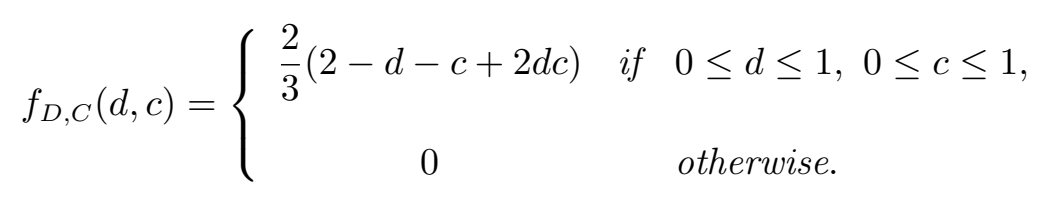

For the sake of clarity in the presentation, in Figure 3.10 the 1-PDF $f_{1}(x, t)$ given by (3.29) is shown for different values of $t$. From it, one infers that the variability of solution SP of IVP (3.1) tends to increases as time $t$ goes on. 

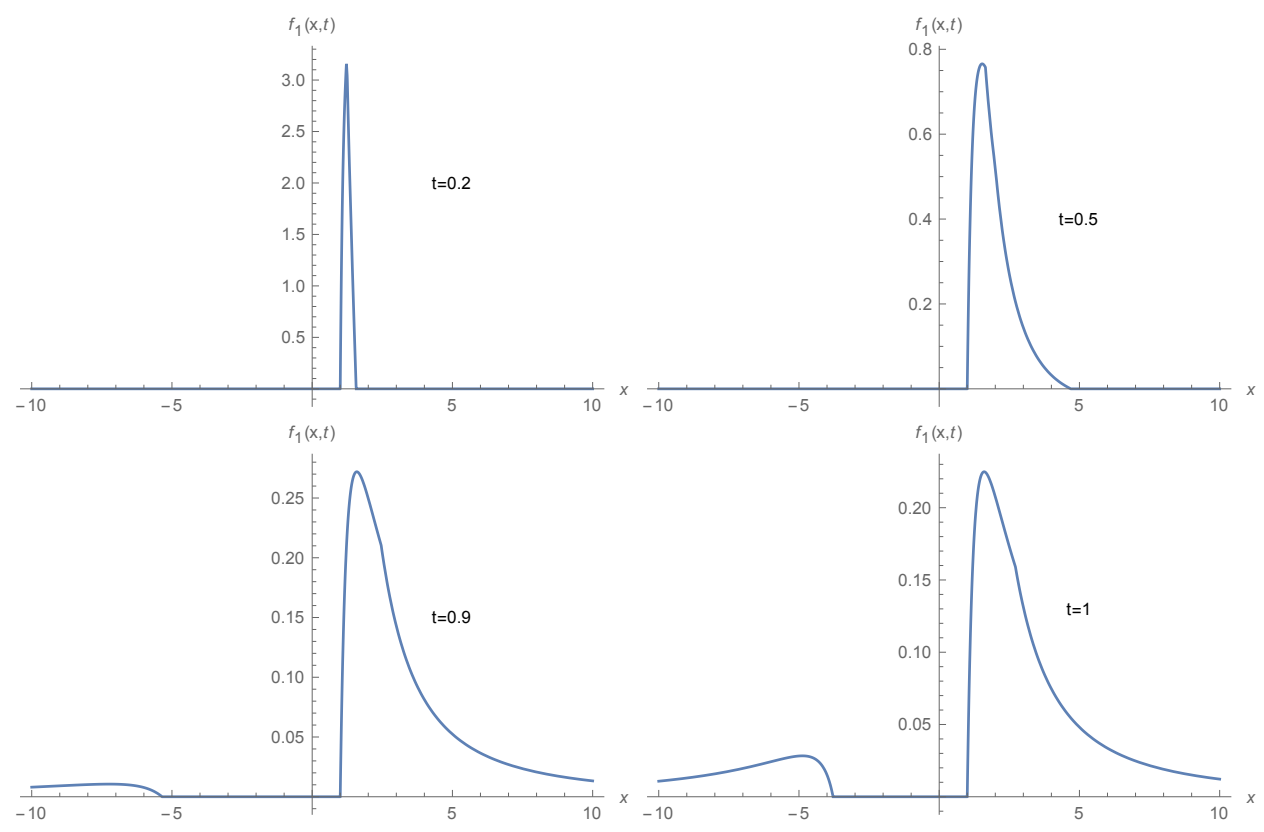

Figure 3.10: Plot of the 1-PDF $f_{1}(x, t)$ in Example 3.9 at different values of $t \in\{0.2,0.5$, $0.9,1\}$ in the case that $x_{0}=1$ and $(D, C)$ has the joint PDF given by (3.30).

\section{Case III.7: $\left(\mathrm{X}_{0}, \mathrm{D}, \mathrm{C}\right)$ is a random vector}

In this last case, in IVP (3.2) it is assumed that all the inputs $\left(X_{0}, D, C\right)$ are RVs whose joint PDF is $f_{X_{0}, D, C}\left(x_{0}, d, c\right)$. Taking into account Table 3.1 and (3.4), this corresponds to

$$
\left.\begin{array}{l}
\dot{Z}(t)=A Z(t)+B, \\
Z(0)=Z_{0},
\end{array}\right\} \quad Z_{0}=\frac{1}{X_{0}}, \quad B=-D, \quad A=-C
$$

Let $t>0$ be fixed, then applying [16, Equation (157)] the PDF of solution SP of IVP (3.31) evaluated at that $t$ is given by

$$
\begin{aligned}
f_{Z}(z)= & \iint_{\mathcal{D}\left(Z_{3}, Z_{2}\right)} f_{Z_{0}, B, A}\left(-\frac{(z-\xi-\eta) \eta}{\xi},-\frac{\eta}{t} \log \left(\frac{-\xi}{\eta}\right), \frac{1}{t} \log \left(\frac{-\xi}{\eta}\right)\right) \\
& \times \frac{|\eta|}{\xi^{2}} \frac{1}{t^{2}}\left|\log \left(-\frac{\xi}{\eta}\right)\right| \mathrm{d} \xi \mathrm{d} \eta
\end{aligned}
$$


where $Z_{2}=\mathrm{e}^{A t} B / A$ and $Z_{3}=-B / A$. Now, the PDF $f_{Z}(z)$ will be expressed as a function of $\left(X_{0}, D, C\right)$ by applying Proposition 2.5 to $U_{1}=X_{0}, U_{2}=D$, $U_{3}=C, V_{1}=Z_{0}, V_{2}=B$ and $V_{3}=A$,

$$
\begin{aligned}
f_{Z}(z)= & \iint_{\mathcal{D}\left(Z_{3}, Z_{2}\right)} f_{X_{0}, D, C}\left(-\frac{\xi}{(z-\xi-\eta) \eta}, \frac{\eta}{t} \log \left(-\frac{\xi}{\eta}\right),-\frac{1}{t} \log \left(-\frac{\xi}{\eta}\right)\right) \\
& \times \frac{|\eta|}{\eta^{2}} \frac{1}{(z-\xi-\eta)^{2} t^{2}}\left|\log \left(-\frac{\xi}{\eta}\right)\right| \mathrm{d} \xi \mathrm{d} \eta .
\end{aligned}
$$

In order to represent (3.32) as a function of $X$, Proposition 2.2 is applied taking into account that $X=1 / Z$

$$
\begin{aligned}
f_{X}(x)= & \frac{1}{x^{2}} f_{Z}\left(\frac{1}{x}\right) \\
= & \iint_{\mathcal{D}\left(Z_{3}, Z_{2}\right)} f_{X_{0}, D, C}\left(-\frac{x \xi}{(1-x \xi-x \eta) \eta}, \frac{\eta}{t} \log \left(-\frac{\xi}{\eta}\right),-\frac{1}{t} \log \left(-\frac{\xi}{\eta}\right)\right) \\
& \times \frac{|\eta|}{\eta^{2}} \frac{1}{(1-x \xi-x \eta)^{2} t^{2}}\left|\log \left(-\frac{\xi}{\eta}\right)\right| \mathrm{d} \xi \mathrm{d} \eta .
\end{aligned}
$$

As $Z_{2}=\mathrm{e}^{A t} B / A=D /\left(C \mathrm{e}^{C t}\right)$ and $Z_{3}=-B / A=-D / C$, the 1-PDF of solution SP $X(t)$ to IVP (3.1) is given by

$$
\begin{aligned}
f_{1}(x, t)= & \iint_{\mathcal{D}\left(\frac{-D}{C}, \frac{D}{C \mathrm{e}^{C t}}\right)} f_{X_{0}, D, C}\left(\frac{-x \xi}{(1-x \xi-x \eta) \eta}, \frac{\eta}{t} \log \left(\frac{-\xi}{\eta}\right), \frac{-1}{t} \log \left(\frac{-\xi}{\eta}\right)\right) \\
& \times \frac{|\eta|}{\eta^{2}} \frac{1}{(1-x \xi-x \eta)^{2} t^{2}}\left|\log \left(-\frac{\xi}{\eta}\right)\right| \mathrm{d} \xi \mathrm{d} \eta
\end{aligned}
$$

If $t=0$, as $X(0)=X_{0}$, the 1-PDF of $X(t)$ is the $(D, C)$-marginal PDF of $f_{X_{0}, D, C}\left(x_{0}, d, c\right)$

$$
f_{1}(x, 0)=\iint_{\mathcal{D}(C, D)} f_{X_{0}, D, C}\left(x_{0}, d, c\right) \mathrm{d} d \mathrm{~d} c .
$$


Example 3.10 Let us assume that random vector $\left(X_{0}, D, C\right)$ has a multivariate Gaussian distribution with mean vector $\boldsymbol{\mu}$ and variance-covariance matrix $\boldsymbol{\Sigma}$ defined as follows

$$
\boldsymbol{\mu}=\left(\mu_{X_{0}}, \mu_{D}, \mu_{C}\right)=(1,1,1), \quad \boldsymbol{\Sigma}=\frac{1}{10}\left(\begin{array}{ccc}
4 & 4 & 4 \\
1 & 4 & 1 \\
1 & 1 & 2
\end{array}\right)
$$

Figure 3.11 shows the 1-PDF $f_{1}(x, t)$ given by (3.33) at different values of $t$. From it, one observes that the variability of solution SP of IVP (3.1) reduces as $t$ increases.
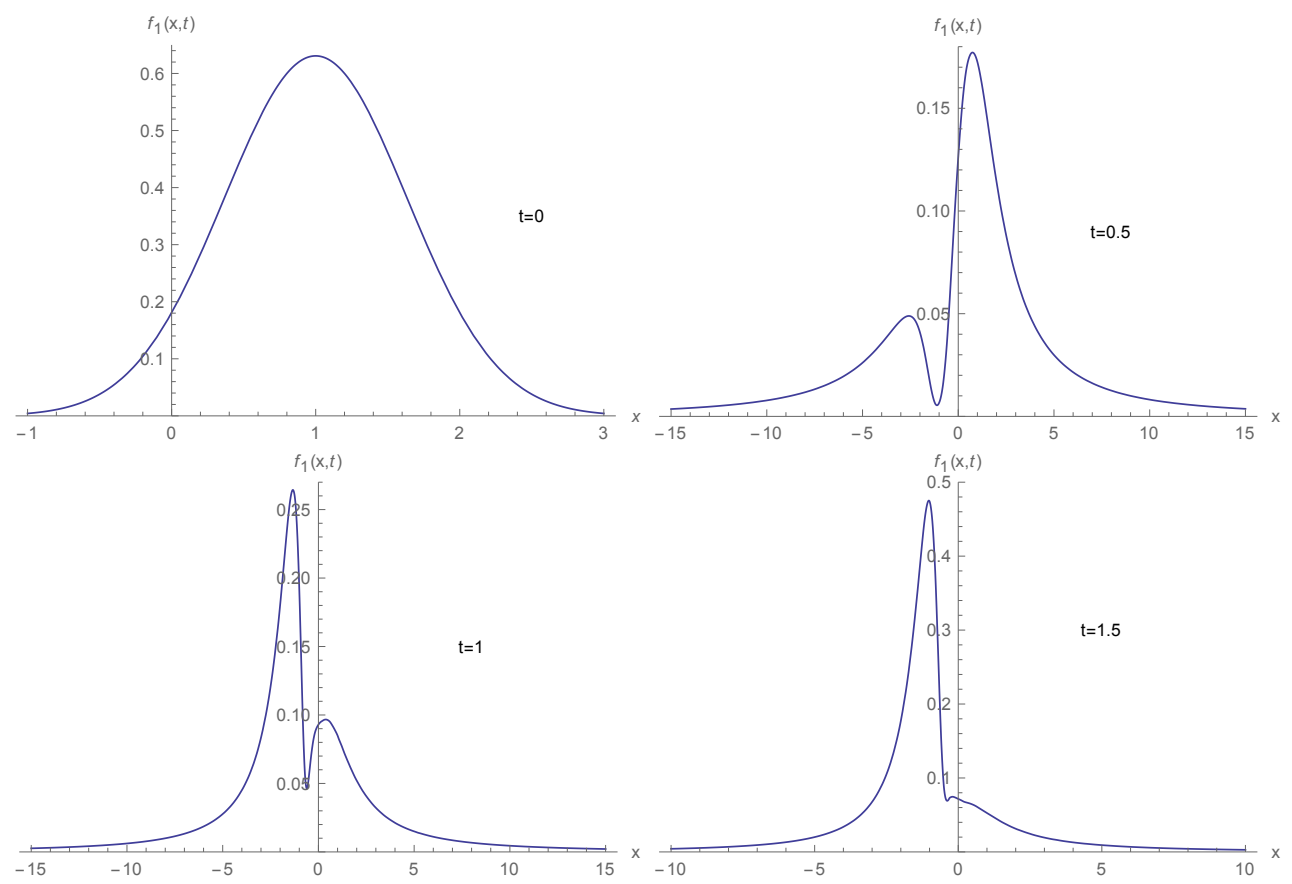

Figure 3.11: Plot of the 1-PDF $f_{1}(x, t)$ in Example 3.10 at different values of $t \in\{0,0.5,1$, $1.5\}$ in the case that $\left(X_{0}, D, C\right)$ has a multivariate Gaussian distribution with mean vector $\boldsymbol{\mu}$ and variance-covariance matrix $\boldsymbol{\Sigma}$ given by (3.34). 


\subsection{Conclusions}

In this chapter it has been shown that the Random Variable Transformation method together with linearization techniques can be used successfully to obtain explicit formulas for the first probability density function of solution stochastic process of non-linear random differential equations. The study has been conducted through homogeneous Riccati differential equation, although it opens the possibility to be extended to other significant types of non-linear continuous models. The usefulness of applying both techniques to deal with these class of problems has been shown through a number of illustrative examples. 


\section{Chapter 4}

\section{Random SIS-type epidemiological models}

This chapter provides a complete probabilistic description of SIS-type epidemiological models where all input parameters (contagion rate, recovery rate and initial conditions) are assumed to be random variables. Applying the Random Variable Transformation technique, the first probability density function, the mean and the variance functions, as well as confidence intervals associated to solution of SIS-type epidemiological models, are determined. It is done under the general hypothesis that model random inputs have a joint probability density function. The distributions to describe the time until a given proportion of population remains susceptible and infected are also determined. A probabilistic description of the socalled basic reproductive number is also included. The theoretical results are applied to an illustrative example showing good fitting. In this example a probabilistic study of the spread smoking is Spain by applying real data is performed. 


\subsection{Introduction}

This chapter is a continuation of contribution [18]. In [18], a comprehensive probabilistic description of solution to random SI-type epidemiological models is provided. SI-type epidemiological models are appropriate to describe the dynamics from susceptible (S) to Infected (I) for some stages of epidemics. For example, SI model has been used to describe the early stages of the AIDS disease and epidemics with a long incubation period. For these kind of diseases, births and deaths or other transitions have no significant effect on the epidemics [54]. They are also useful to model the spread of new technologies, where potential customers are identified with the susceptible subpopulation and, the remainder of the individuals who have already adopted the new technology, corresponds to the infected subpopulation [18, 77, 96]. However, SI-models are not appropriate for most epidemics which involve transitions from infected to susceptible. As it shall be justified below, this has motivated the consideration of the so-called SIS-type epidemiological models [46]. The aim of this chapter is to extend the results obtained in [18] for SIS-type epidemiological models. This extension entails, among other aspects, the generalization to the random framework of the so-called reproductive number, which does not appear in dealing with SI-type epidemiological models. The approach is based on RVT technique, and its consideration is justified because a full probabilistic description of solution SP for random SIS-type epidemiological models can be obtained under very general assumptions. Indeed, as it shall be seen later in the theoretical development, statistical dependence of all input parameters involved into SIS-model via any plausible joint PDF will be considered. As it was pointed out in the introduction, this is a significant advantage that other available approaches, such as SIS-type epidemiological models based on Itô stochastic differential equations, do not possess.

SIS-type epidemiological models constitute mathematical representations to describe the spread by individual-to-individual contact of infectious diseases $[11,47]$. SIS models are useful for modelling diseases whose infection does not confer immunity, that is, any susceptible (S) who has been infected (I) can recover from the disease and then becoming susceptible (S) again. This is usually represented as $S \rightarrow I \rightarrow S$ (Susceptible-Infected-Susceptible). Examples of diseases that have been modelled by SIS models include gonorrhea, meningitis, streptococcal sore throat, etc. [21, 46, 48]. SIS epidemiological models have also been used to model the dynamics of unhealthy lifestyle habits such as the excess of weight [30]. In spite of their mathematical simplicity, SIS models constitute the basis of more refined and sophisticated models where, for 
instance, mode of transmission, resistance, environmental and cultural factors, and further diseases characteristics can be considered.

SIS models are formulated as IVPs based on non-linear systems of differential equations of the form

$$
\left\{\begin{array}{l}
\dot{S}(t)=-\beta S(t) I(t)+\gamma I(t), \quad t>0 \\
\dot{I}(t)=\beta S(t) I(t)-\gamma I(t),
\end{array}\right.
$$

with initial conditions

$$
S(0)=S_{0}, \quad I(0)=I_{0},
$$

where, $S(t)$ and $I(t)$ denote the proportion, or equivalently, the percentage of susceptibles and infected at the time instant $t$, respectively. At the initial time instant, $t=0$, these values correspond to $S_{0}$ and $I_{0}$, respectively. It is assumed that

$$
S(t)+I(t)=1, \quad \forall t \geq 0,
$$

which means that all the individuals of population are classified as either susceptible or infected. Parameters $\beta>0$ and $\gamma>0$ denote the rate of decline in the percentage of susceptibles and the rate of infected that recover from the disease, respectively. In Figure 4.1, a flow diagram of SIS model is depicted.

The consideration of births and deaths, usually referred to as vital dynamics, could be included in model (4.1)-(4.3). However, the behaviour of solutions of the new corresponding model is similar when vital dynamics are omitted in deterministic models [46]. This motivates the analysis of the simpler model (4.1)-(4.3) as a first step. The inclusion of vital dynamics using RVs to model births and deaths is a very interesting problem that requires its own subsequent development, although it would probably benefit from the ideas presented here.

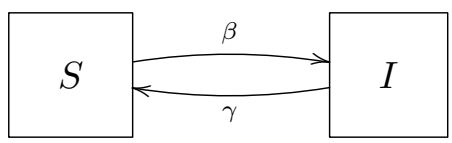

Figure 4.1: Flow diagram representation of SIS model (4.1)-(4.2). 
From a practical point of view, it is more realistic to regard that input parameters $S_{0}, \gamma$ and $\beta$ are RVs rather than deterministic values due to the inherent complexity and uncertainty that involve their determination and interpretation. Throughout this chapter all random variables/vectors are assumed to be absolutely continuous in order to guarantee the existence of their associated PDFs. Initial condition $S_{0}$ represents the percentage of susceptibles at the beginning. In practice, this value is usually set after sampling the target population, hence it has got variability. Since $S_{0}$ represents a percentage, it lies between 0 and 1, and it could be better modelled by a RV like a Uniform RV on the interval $(0,1)$, or a Beta RV, which is more flexible and generalizes $(0,1)$ Uniform RV. Positive parameters $\gamma$ and $\beta$ embed complex factors related to the recovery rate from the disease and the contagion rate, respectively. Notice that, in practice, they are both not known with certainty. This motivates that positive probability distributions, such as Exponential, Gamma, or even Gaussian truncated on positive domain, might be adequate candidates for modelling input parameters $\gamma$ and $\beta$. As will be seen later, these type of probability distributions will be assumed in the illustrative example shown in this chapter. The consideration of randomness in SIS-type epidemiological models has been undertaken mainly using Itô-type stochastic differential equations [24, 25].

The chapter is organized as follows. Section 4.2 is devoted to provide a complete probabilistic description of random SIS-type epidemiological model (4.1)(4.2) under general hypotheses regarding the probability distribution of random inputs. For the sake of clarity, this section is divided into several pieces. Subsection 4.2.1 deals with the computation of the first probability distributions of the percentages of susceptibles and infected. The determination of mean and variance functions as well as confidence intervals for the percentages of susceptibles is established in Subsection 4.2.2. Subsection 4.2.3 is addressed to compute the distributions of time until a given proportion of population remains susceptible and infected, respectively. Subsection 4.2 .4 ends by providing a probabilistic description of the basic reproductive number, which plays a key role in dealing with the behaviour of a disease in the long run. In Section 4.3, all the theoretical results obtained in Section 4.2 are applied to study, using available real data, the evolution of Spanish non-smokers men older than 16 years old under SIS model approach. Conclusions are drawn in Section 4.4. 


\subsection{Probabilistic solution}

This section is devoted to determine the probabilistic solution of random SIS model (4.1)-(4.3). This means the computation of the 1-PDF of each component, $S(t)$ and $I(t)$, of its solution, $(S(t), I(t))$, as well as its main statistical properties, such as the mean and variance functions and confidence intervals. To complete useful information in practical situations, the PDFs of time until a given proportion of population remains susceptible/infected and the so-called reproductive number will be also investigated.

To achieve these goals, first, taking into account (4.3), it is convenient to rewrite (4.1)-(4.2) as the following random non-linear IVP

$$
\left\{\begin{array}{l}
\dot{S}(t)=\beta(S(t))^{2}-(\gamma+\beta) S(t)+\gamma, \quad t>0 \\
S(0)=S_{0}
\end{array}\right.
$$

As it has been motivated in the introduction of the chapter, throughout the exposition it will be assumed that input parameters $S_{0}, \gamma$ and $\beta$ are RVs, and

$$
\begin{aligned}
& \mathcal{D}\left(S_{0}\right)=\left\{s_{0}=S_{0}(\omega), \omega \in \Omega: 0 \leq s_{0,1} \leq s_{0} \leq s_{0,2} \leq 1\right\} \\
& \mathcal{D}(\gamma)=\left\{\gamma=\gamma(\omega), \omega \in \Omega: 0 \leq \gamma_{1} \leq \gamma \leq \gamma_{2}\right\} \\
& \mathcal{D}(\beta)=\left\{\beta=\beta(\omega), \omega \in \Omega: 0 \leq \beta_{1} \leq \beta \leq \beta_{2}\right\}
\end{aligned}
$$

will denote their respective domains. Hereinafter, $f_{S_{0}, \gamma, \beta}\left(s_{0}, \gamma, \beta\right)$ will indicate the joint PDF of input random vector $\left(S_{0}, \gamma, \beta\right)$. At this point, it is important to underline that in order to provide more generality at the results that will be obtained later, the joint $\operatorname{PDF} f_{S_{0}, \gamma, \beta}\left(s_{0}, \gamma, \beta\right)$ will be considered, although in the example input parameters $S_{0}, \gamma$ and $\beta$ will be assumed pairwise independent RVs. For the sake of clarity in the presentation, it is assumed that the domain of random vector $\left(S_{0}, \gamma, \beta\right)$ is the product of intervals given by (4.5) rather than any arbitrary subset of $\mathbb{R}^{3}$.

From a theoretical point of view is important to guarantee the uniqueness of solution to system of random differential equations (4.1)-(4.3), or equivalently, the uniqueness of solution $S(t)$ to IVP (4.4) on a finite interval, $t \in[0, a], a>0$. To achieve this goal, [85, Theorem 5.1.2., p.118] will be applied, which is a natural generalization of classical Picard theorem based upon convergence of successive approximations. This result is stated in terms of a strong stochastic convergence, namely, m.s. convergence. As it was explained in the preliminaries, this type of stochastic convergence is defined on the Hilbert space $\left(\mathrm{L}_{2},\|\cdot\|\right)$ 
of the 2-RVs, where the norm is defined in terms of the expectation operator: $\|X\|_{2}=\left(\mathbb{E}\left[X^{2}\right]\right)^{1 / 2}$.

Following the standard notation introduced in [85, Theorem 5.1.2., p.118], let us consider the right-hand side of random differential equation (4.4), i.e., $f(S, t)=\beta S^{2}-(\gamma+\beta) S+\gamma$, and the pair defining random initial condition $\left(t_{0}, X_{0}\right)=\left(0, S_{0}\right)$. In accordance with (4.5), let us assume that $\beta$ and $\gamma$ have bounded realizations, hence they are 2-RVs. Moreover, observe that $S$ is also a 2 -RV because $0 \leq S(\omega) \leq 1$ for each $\omega \in \Omega$. First, it is needed to check the following m.s. Lipschitz condition

$$
\left\|f\left(S_{1}, t\right)-f\left(S_{2}, t\right)\right\|_{2} \leq k(t)\left\|S_{1}-S_{2}\right\|_{2}, \quad \text { where } \int_{0}^{a} k(t) \mathrm{d} t<\infty .
$$

For convenience, let us denote by $\delta=\max \left\{\beta_{2}, \gamma_{2}\right\}$, being $\beta_{2}$ and $\gamma_{2}$ the upper bounds defined in (4.5). Since $S_{1}$ and $S_{2}$ are functions of the random inputs $S_{0}, \beta$ and $\gamma$, and by (4.3), $0 \leq S_{1}(\omega)+S_{2}(\omega) \leq 2$ for all $\omega \in \Omega$, one gets

$$
\begin{aligned}
\left(\left\|f\left(S_{1}, t\right)-f\left(S_{2}, t\right)\right\|_{2}\right)^{2}= & \left(\left\|\beta\left(\left(S_{1}\right)^{2}-\left(S_{2}\right)^{2}\right)-(\gamma+\beta)\left(S_{1}-S_{2}\right)\right\|_{2}\right)^{2} \\
= & \left(\left\|\left(\beta\left(S_{1}+S_{2}\right)-(\gamma+\beta)\right)\left(S_{1}-S_{2}\right)\right\|_{2}\right)^{2} \\
= & \mathbb{E}\left[\left(\beta\left(S_{1}+S_{2}\right)-(\gamma+\beta)\right)^{2}\left(S_{1}-S_{2}\right)^{2}\right] \\
= & \int_{\mathcal{D}\left(S_{0}\right)} \int_{\mathcal{D}(\gamma)} \int_{\mathcal{D}(\beta)}\left(\beta\left(S_{1}+S_{2}\right)-(\gamma+\beta)\right)^{2} \\
& \times\left(S_{1}-S_{2}\right)^{2} f_{S_{0}, \gamma, \beta}\left(s_{0}, \gamma, \beta\right) \mathrm{d} \beta \mathrm{d} \gamma \mathrm{d} s_{0} \\
\leq & \int_{\mathcal{D}\left(S_{0}\right)} \int_{\mathcal{D}(\gamma)} \int_{\mathcal{D}(\beta)}(2 \beta-(\gamma+\beta))^{2} \\
& \times\left(S_{1}-S_{2}\right)^{2} f_{S_{0}, \gamma, \beta}\left(s_{0}, \gamma, \beta\right) \mathrm{d} \beta \mathrm{d} \gamma \mathrm{d} s_{0} \\
= & \int_{\mathcal{D}\left(S_{0}\right)} \int_{\mathcal{D}(\gamma)} \int_{\mathcal{D}(\beta)}(\beta-\gamma)^{2} \\
& \times\left(S_{1}-S_{2}\right)^{2} f_{S_{0}, \gamma, \beta}\left(s_{0}, \gamma, \beta\right) \mathrm{d} \beta \mathrm{d} \gamma \mathrm{d} s_{0}
\end{aligned}
$$




$$
\begin{aligned}
& \leq \delta^{2} \int_{\mathcal{D}\left(S_{0}\right)} \int_{\mathcal{D}(\gamma)} \int_{\mathcal{D}(\beta)}\left(S_{1}-S_{2}\right)^{2} f_{S_{0}, \gamma, \beta}\left(s_{0}, \gamma, \beta\right) \mathrm{d} \beta \mathrm{d} \gamma \mathrm{d} s_{0} \\
& =\delta^{2} \mathbb{E}\left[\left(S_{1}-S_{2}\right)^{2}\right] \\
& =\delta^{2}\left(\left\|S_{1}-S_{2}\right\|_{2}\right)^{2} .
\end{aligned}
$$

Therefore, Lipschitz condition (4.6) holds true taking $k(t)=\delta$. Notice that

$$
\int_{0}^{a} k(t) \mathrm{d} t=\int_{0}^{a} \delta \mathrm{d} t=\delta a<\infty
$$

The second condition to be checked is that the function $f(S, t)$ transforms 2RVs into 2-RVs. This is a direct consequence of the previous development, the triangular inequality and the fact that $\gamma$ and $S$ are also 2-RVs

$$
\begin{aligned}
\|f(S, t)\|_{2} & =\left\|\beta S^{2}-(\beta+\gamma) S+\gamma\right\|_{2} \\
& \leq\left\|\beta S^{2}-(\beta+\gamma) S\right\|_{2}+\|\gamma\|_{2} \leq \delta\|S\|_{2}+\|\gamma\|_{2}<\infty
\end{aligned}
$$

\subsubsection{First probability density function}

The aim of this subsection is to obtain the 1-PDF, $f_{1}(s, t)$, of solution SP, $S(t)$, of random IVP (4.4). This function provides a full probabilistic description of the percentage of susceptibles at every time instant $t$. Once this goal has been achieved, using (4.3), the 1-PDF of the percentage of infected $I(t)$ will also be provided.

First, notice that solution SP $S(t)$ of random IVP (4.4) is given by

$$
S(t)=\frac{\gamma\left(1-S_{0}\right)+\left(S_{0} \beta-\gamma\right) \mathrm{e}^{(\gamma-\beta) t}}{\beta\left(1-S_{0}\right)+\left(S_{0} \beta-\gamma\right) \mathrm{e}^{(\gamma-\beta) t}}, \quad t \geq 0
$$

Now, fix $t \geq 0$ and apply RVT method, Theorem 2.1, with the following choice

$$
\mathbf{X}=\left(S_{0}, \gamma, \beta\right), \quad \mathbf{Y}=\left(Y_{1}, Y_{2}, Y_{3}\right), \quad \mathbf{Y}=\mathbf{r}(\mathbf{X})
$$

being 


$$
Y_{1}=\frac{\gamma\left(1-S_{0}\right)+\left(S_{0} \beta-\gamma\right) \mathrm{e}^{(\gamma-\beta) t}}{\beta\left(1-S_{0}\right)+\left(S_{0} \beta-\gamma\right) \mathrm{e}^{(\gamma-\beta) t}}, \quad Y_{2}=\gamma, \quad Y_{3}=\beta
$$

Isolating $S_{0}, \beta$ and $\gamma$, one gets the inverse mapping of $\mathbf{r}$

$$
S_{0}=\frac{Y_{2}+\mathrm{e}^{\left(Y_{2}-Y_{3}\right) t}\left(-1+Y_{1}\right) Y_{2}-Y_{1} Y_{3}}{Y_{2}+\mathrm{e}^{\left(Y_{2}-Y_{3}\right) t}\left(-1+Y_{1}\right) Y_{3}-Y_{1} Y_{3}}, \quad \gamma=Y_{2}, \quad \beta=Y_{3} .
$$

Thus, in accordance with (2.16)-(2.17), the joint PDF of random vector $\left(Y_{1}, Y_{2}\right.$, $\left.Y_{3}\right)$ is given by

$$
\begin{aligned}
f_{Y_{1}, Y_{2}, Y_{3}}\left(y_{1}, y_{2}, y_{3}\right)= & f_{S_{0}, \gamma, \beta}\left(\frac{y_{2}+\mathrm{e}^{\left(y_{2}-y_{3}\right) t}\left(-1+y_{1}\right) y_{2}-y_{1} y_{3}}{y_{2}+\mathrm{e}^{\left(y_{2}-y_{3}\right) t}\left(-1+y_{1}\right) y_{3}-y_{1} y_{3}}, y_{2}, y_{3}\right) \\
& \times \frac{\mathrm{e}^{\left(y_{2}-y_{3}\right) t}\left(y_{2}-y_{3}\right)^{2}}{\left(y_{2}+\mathrm{e}^{\left(y_{2}-y_{3}\right) t}\left(-1+y_{1}\right) y_{3}-y_{1} y_{3}\right)^{2}},
\end{aligned}
$$

where $J_{3}=\left(\mathrm{e}^{\left(y_{2}-y_{3}\right) t}\left(y_{2}-y_{3}\right)^{2}\right) /\left(y_{2}+\mathrm{e}^{\left(y_{2}-y_{3}\right) t}\left(-1+y_{1}\right) y_{3}-y_{1} y_{3}\right)^{2}>0$ is the Jacobian obtained from the inverse mapping of $\mathbf{r}$ defined according to (4.8). Notice that $J_{3} \neq 0$ since $\gamma(\omega) \neq \beta(\omega)$ a.s.

Therefore, the PDF of RV $S=S(t)$ with $t$ fixed, is the $(\gamma, \beta)$-marginal PDF of the joint PDF (4.9), i.e.,

$$
\begin{aligned}
f_{S}(s)= & \int_{\mathcal{D}(\gamma)} \int_{\mathcal{D}(\beta)} f_{S_{0}, \gamma, \beta}\left(\frac{\xi+\mathrm{e}^{(\xi-\eta) t}(-1+s) \xi-s \eta}{\xi+\mathrm{e}^{(\xi-\eta) t}(-1+s) \eta-s \eta}, \xi, \eta\right) \\
& \times \frac{\mathrm{e}^{(\xi-\eta) t}(\xi-\eta)^{2}}{\left(\xi+\mathrm{e}^{(\xi-\eta) t}(-1+s) \eta-s \eta\right)^{2}} \mathrm{~d} \eta \mathrm{d} \xi .
\end{aligned}
$$


As the previous development is valid for every $t \geq 0$, the 1-PDF of solution SP $S(t)$ of IVP (4.4) is given by

$$
\begin{aligned}
f_{1}(s, t)= & \int_{\mathcal{D}(\gamma)} \int_{\mathcal{D}(\beta)} f_{S_{0}, \gamma, \beta}\left(\frac{\xi+\mathrm{e}^{(\xi-\eta) t}(-1+s) \xi-s \eta}{\xi+\mathrm{e}^{(\xi-\eta) t}(-1+s) \eta-s \eta}, \xi, \eta\right) \\
& \times \frac{\mathrm{e}^{(\xi-\eta) t}(\xi-\eta)^{2}}{\left(\xi+\mathrm{e}^{(\xi-\eta) t}(-1+s) \eta-s \eta\right)^{2}} \mathrm{~d} \eta \mathrm{d} \xi .
\end{aligned}
$$

Now, denoting $S=S(t)$ and $I=I(t)$ with $t$ fixed, using relationship (4.3) and Proposition 2.6 with the identification $U=S, V=I, a=-1 \neq 0$ and $b=1$, from (4.10) one obtains the PDF of RV $I$ and, hence straightforwardly the 1-PDF of the percentage of infected

$$
I(t)=\frac{(\beta-\gamma)\left(1-S_{0}\right) \mathrm{e}^{(\beta-\gamma) t}}{\beta\left(1-S_{0}\right) \mathrm{e}^{(\beta-\gamma) t}+S_{0} \beta-\gamma}, \quad t \geq 0,
$$

which is given by

$$
\begin{aligned}
f_{1}(i, t)= & \int_{\mathcal{D}(\gamma)} \int_{\mathcal{D}(\beta)} f_{S_{0}, \gamma, \beta}\left(\frac{\xi-\eta-\mathrm{e}^{(\xi-\eta) t} i \xi+i \eta}{\xi-\eta-\mathrm{e}^{(\xi-\eta) t} i \eta+i \eta}, \xi, \eta\right) \\
& \times \frac{\mathrm{e}^{(\xi-\eta) t}(\xi-\eta)^{2}}{\left(\xi-\eta-\mathrm{e}^{(\xi-\eta) t} i \eta+i \eta\right)^{2}} \mathrm{~d} \eta \mathrm{d} \xi
\end{aligned}
$$

\subsubsection{Mean and variance functions. Confidence intervals}

In the context of the applications, the usefulness of the 1-PDF is manifested in dealing with the computation of the main statistical information associated to solution $(S(t), I(t))$. Specifically, the mean and the variance functions of $S(t)$ are defined by

$$
\begin{aligned}
& \mu_{S}(t)=\mathbb{E}[S(t)]=\int_{-\infty}^{\infty} s f_{1}(s, t) \mathrm{d} s \\
& \left(\sigma_{S}(t)\right)^{2}=\mathbb{V}[S(t)]=\int_{-\infty}^{\infty} s^{2} f_{1}(s, t) \mathrm{d} s-\left(\mu_{S}(t)\right)^{2},
\end{aligned}
$$


where $f_{1}(s, t)$ is given by (4.11). The same can be stated to regard the computation of $\mu_{I}(t)$ and $\sigma_{I}(t)$ changing $f_{1}(s, t)$ by $f_{1}(i, t)$, which has been determined in (4.13). Apart from computing the mean statistical behaviour of solution SP of SIS model and its variability, for $\hat{t} \geq 0$ fixed, it can be interesting to determine the probability that the percentage of susceptibles lies within a specific interval, say, $\left[s_{1}(\hat{t}), s_{2}(\hat{t})\right]=\left[\hat{s}_{1}, \hat{s}_{2}\right]$. It can be easily determined, once $f_{1}(s, t)$ has been computed, by the following integral

$$
\mathbb{P}\left[\left\{\omega \in \Omega: S(\hat{t} ; \omega) \in\left[\hat{s}_{1}, \hat{s}_{2}\right]\right\}\right]=\int_{\hat{s}_{1}}^{\hat{s}_{2}} f_{1}(s, \hat{t}) \mathrm{d} s .
$$

Regarding model validation and predictions, the 1-PDF is also very useful to construct probabilistic intervals for any $(1-\alpha) \times 100 \%$ confidence level. For instance, fixed $\alpha \in(0,1)$, for each $\hat{t} \geq 0$ fixed, one can determine $s_{1}(\hat{t})$ and $s_{2}(\hat{t})$ such that

$$
\int_{0}^{s_{1}(\hat{t})} f_{1}(s, \hat{t}) \mathrm{d} s=\frac{\alpha}{2}=\int_{s_{2}(\hat{t})}^{1} f_{1}(s, \hat{t}) \mathrm{d} s
$$

where

$$
1-\alpha=\mathbb{P}\left[\left\{\omega \in \Omega: S(\hat{t} ; \omega) \in\left[s_{1}(\hat{t}), s_{2}(\hat{t})\right]\right\}\right]=\int_{s_{1}(\hat{t})}^{s_{2}(\hat{t})} f_{1}(s, \hat{t}) \mathrm{d} s .
$$

Commonly $\alpha=0.05$, which means that $95 \%$ confidence intervals are built.

Notice that analogous expressions to $(4.15)-(4.17)$ can be given for the percentage of infected simply interchanging $f_{1}(s, \hat{t})$ by $f_{1}(i, \hat{t})$, where the latter function is defined by (4.13).

\subsubsection{Distribution of time until a given proportion of population remains susceptible}

So far the distribution for the percentage of susceptibles (and infected) at every time instant $t$ has been determined. From an applied standpoint, it is very helpful to compute when the percentage of susceptibles in population will attain a specific level. With this end, let us denote by $T_{S}$ the time until a given proportion of population, $\rho_{S}$, remains susceptible, i.e., $\rho_{S}=S(T)$. Then, isolating $T_{S}$ from the exact solution (4.7) of IVP (4.4) one gets 


$$
T_{S}=\frac{1}{\gamma-\beta} \log \left(\frac{\left(1-S_{0}\right)\left(\beta \rho_{S}-\gamma\right)}{\left(1-\rho_{S}\right)\left(\beta S_{0}-\gamma\right)}\right)
$$

Now, applying Theorem 2.1 to

$$
\mathbf{X}=\left(S_{0}, \gamma, \beta\right), \quad \mathbf{Y}=\left(Y_{1}, Y_{2}, Y_{3}\right), \quad \mathbf{Y}=\mathbf{r}(\mathbf{X})
$$

being

$$
Y_{1}=\frac{1}{\gamma-\beta} \log \left(\frac{\left(1-S_{0}\right)\left(\beta \rho_{S}-\gamma\right)}{\left(1-\rho_{S}\right)\left(\beta S_{0}-\gamma\right)}\right), \quad Y_{2}=\gamma, \quad Y_{3}=\beta
$$

Isolating $S_{0}, \gamma$ and $\beta$, one gets the inverse mapping of $\mathbf{r}$

$$
S_{0}=\frac{Y_{2}\left(1+\mathrm{e}^{Y_{1}\left(Y_{2}-Y_{3}\right)}\left(-1+\rho_{S}\right)\right)-Y_{3} \rho_{S}}{Y_{2}+Y_{3}\left(\mathrm{e}^{Y_{1}\left(Y_{2}-Y_{3}\right)}\left(-1+\rho_{S}\right)-\rho_{S}\right)}, \quad \gamma=Y_{2}, \quad \beta=Y_{3}
$$

Then, applying expressions $(2.16)-(2.17)$ and considering that $\rho_{S} \in(0,1)$, the joint $\mathrm{PDF}$ of random vector $\left(Y_{1}, Y_{2}, Y_{3}\right)$ is given by

$$
\begin{aligned}
f_{Y_{1}, Y_{2}, Y_{3}}\left(y_{1}, y_{2}, y_{3}\right)= & f_{S_{0}, \gamma, \beta}\left(\frac{y_{2}\left(1+\mathrm{e}^{y_{1}\left(y_{2}-y_{3}\right)}\left(-1+\rho_{S}\right)\right)-y_{3} \rho_{S}}{y_{2}+y_{3}\left(\mathrm{e}^{y_{1}\left(y_{2}-y_{3}\right)}\left(-1+\rho_{S}\right)-\rho_{S}\right)}, y_{2}, y_{3}\right) \\
& \times \frac{\mathrm{e}^{y_{1}\left(y_{2}-y_{3}\right)}\left(y_{2}-y_{3}\right)^{2}\left(1-\rho_{S}\right)\left|y_{2}-y_{3} \rho_{S}\right|}{\left(y_{2}+y_{3}\left(\mathrm{e}^{y_{1}\left(y_{2}-y_{3}\right)}\left(-1+\rho_{S}\right)-\rho_{S}\right)\right)^{2}},
\end{aligned}
$$

where

$$
J_{3}=\frac{\left(\mathrm{e}^{y_{1}\left(y_{2}-y_{3}\right)}\left(y_{2}-y_{3}\right)^{2}\left(-1+\rho_{S}\right)\left(y_{2}-y_{3} \rho_{S}\right)\right)}{\left(y_{2}+y_{3}\left(\mathrm{e}^{y_{1}\left(y_{2}-y_{3}\right)}\left(-1+\rho_{S}\right)-\rho_{S}\right)\right)^{2}}
$$

is the Jacobian obtained from the inverse mapping of $\mathbf{r}$ defined according to (4.18). $J_{3} \neq 0$ since $\gamma(\omega) \neq \beta(\omega), \gamma(\omega) \neq \beta(\omega) \rho_{S}$ w.p. 1 and $\rho_{S} \neq 1$. 
Therefore, taking into account that $\left(Y_{1}, Y_{2}, Y_{3}\right)=\left(T_{S}, \gamma, \beta\right)$, the PDF of RV $T_{S}$ is the $(\gamma, \beta)$-marginal PDF of the joint PDF (4.19)

$$
\begin{aligned}
f_{1}\left(t, \rho_{S}\right)= & \int_{\mathcal{D}(\gamma)} \int_{\mathcal{D}(\beta)} f_{S_{0}, \gamma, \beta}\left(\frac{\xi\left(1+\mathrm{e}^{t(\xi-\eta)}\left(-1+\rho_{S}\right)\right)-\eta \rho_{S}}{\xi+\eta\left(\mathrm{e}^{t(\xi-\eta)}\left(-1+\rho_{S}\right)-\rho_{S}\right)}, \xi, \eta\right) \\
& \times \frac{\mathrm{e}^{t(\xi-\eta)}(\xi-\eta)^{2}\left(1-\rho_{S}\right)\left|\xi-\eta \rho_{S}\right|}{\left(\xi+\eta\left(\mathrm{e}^{t(\xi-\eta)}\left(-1+\rho_{S}\right)-\rho_{S}\right)\right)^{2}} \mathrm{~d} \eta \mathrm{d} \xi .
\end{aligned}
$$

Following an analogous development, one can demonstrate that the distribution of time until a given proportion of population remains infected, $\rho_{I}$, reads

$$
\begin{aligned}
f_{1}\left(t, \rho_{I}\right)= & \int_{\mathcal{D}(\gamma)} \int_{\mathcal{D}(\beta)} f_{S_{0}, \gamma, \beta}\left(\frac{\xi+\eta\left(-1+\rho_{I}\right)-\mathrm{e}^{t(\xi-\eta)}}{\xi-\eta\left(1+\left(-1+\mathrm{e}^{t(\xi-\eta)}\right) \rho_{I}\right)}, \xi, \eta\right) \\
& \times\left|\frac{\mathrm{e}^{t(\xi-\eta)}(\xi-\eta)^{2}\left(\xi+\eta\left(-1+\rho_{I}\right)\right) \rho_{I}}{\left(\xi-\eta\left(1+\left(-1+\mathrm{e}^{t(\xi-\eta)}\right) \rho_{I}\right)\right)^{2}}\right| \mathrm{d} \eta \mathrm{d} \xi .
\end{aligned}
$$

\subsubsection{Basic reproductive number}

In Epidemiology, the basic reproduction number, $R_{0}$, associated to an infection is useful to elucidate whether will spread out or not. In the case of SIS model (4.1)-(4.3), this value and its relationship with the propagation of the epidemic in the long run is given by

$$
R_{0}=\frac{\beta}{\gamma},\left\{\begin{array}{l}
\text { if } \quad R_{0}<1 \equiv \beta<\gamma, \quad \text { the disease will die out as } t \rightarrow+\infty \\
\text { if } \quad R_{0}>1 \equiv \beta>\gamma,
\end{array}\right.
$$

This classification is easily derived from expression (4.12) of $I(t)$, or equivalently of $S(t)$ (see (4.7)), since

$$
\begin{aligned}
& \lim _{t \rightarrow+\infty} I(t)=\lim _{t \rightarrow+\infty} \frac{(\beta-\gamma)\left(1-S_{0}\right) \mathrm{e}^{(\beta-\gamma) t}}{\beta\left(1-S_{0}\right) \mathrm{e}^{(\beta-\gamma) t}+S_{0} \beta-\gamma}=0 \quad \text { if } \beta<\gamma, \\
& \lim _{t \rightarrow+\infty} S(t)=\lim _{t \rightarrow+\infty} \frac{\gamma\left(1-S_{0}\right)+\left(S_{0} \beta-\gamma\right) \mathrm{e}^{(\gamma-\beta) t}}{\beta\left(1-S_{0}\right)+\left(S_{0} \beta-\gamma\right) \mathrm{e}^{(\gamma-\beta) t}}=1 \quad \text { if } \quad \beta<\gamma .
\end{aligned}
$$


Notice that this result is in agreement of its intuitive interpretation, the epidemic will die out when the rate of decline in the percentage of susceptibles be less than the rate of infected that recover from the diseases, in other words, when condition $\beta<\gamma$ holds.

In our context, both $\beta$ and $\gamma$ are assumed to be RVs, so that the requirement for epidemic extinction in the deterministic framework $\beta<\gamma$ means the computation of the following probability in the stochastic scenario

$$
\mathbb{P}[\mathcal{S}], \quad \mathcal{S}=\{\omega \in \Omega: \beta(\omega)<\gamma(\omega)\}=\left\{\omega \in \Omega: R_{0}(\omega)<1\right\}
$$

This key probability can be computed by taking advantage of Proposition 2.7. In fact, using the following identification between the notation for SIS model and the one used in Proposition 2.7

$$
\mathbf{U}=\left(U_{1}, U_{2}\right)=(\gamma, \beta), \quad V=\frac{U_{2}}{U_{1}}=\frac{\beta}{\gamma}=R_{0},
$$

one gets

$$
f_{R_{0}}\left(r_{0}\right)=\int_{\mathcal{D}(\gamma)} f_{\gamma, \beta}\left(\xi, r_{0} \xi\right)|\xi| \mathrm{d} \xi
$$

where $f_{\gamma, \beta}(\cdot, \cdot)$ denotes the $S_{0}$-marginal distribution of the joint PDF of random inputs $S_{0}, \gamma$ and $\beta$. As a consequence, taking into account that $R_{0}(\omega)>0$ for all $\omega \in \Omega$, the target probability (4.21) can be computed as follows

$$
\mathbb{P}[\mathcal{S}]=\int_{0}^{1} \int_{\mathcal{D}(\gamma)} f_{\gamma, \beta}\left(\xi, r_{0} \xi\right)|\xi| \mathrm{d} \xi \mathrm{d} r_{0}
$$

\subsection{An illustrative example}

In this section it has be shown how to take advantage of the theoretical probabilistic results previously obtained for random SIS model (4.1)-(4.3) (or equivalently, (4.4)), in order to study the spread of smoking in Spain.

Example 4.1 SIS-type epidemiological models are useful to describe the spread of diseases whose infection does not confer immunity among the individuals of 
a population which is divided into two groups, susceptible and infected. Many social habits can also be treated by SIS model like smoking. Notice that once a person gives up smoking, he/she could become a smoker again in the future. In this example, SIS model (4.1)-(4.3) is applied to study the evolution of smoking among the Spanish men aged over 16 years old. In this context this population has been divided into two groups, non-smokers (susceptible) and smokers (infected). Table 4.1 collects the percentage of non-smokers, denoted by $S_{j}$, for the available data during the period 1987-2006, that correspond to time values $t_{j}, j \in \mathcal{J}=\{0,6,8,10,14,16,19\}$, respectively. In order to apply model (4.4), it must be chosen specific probability distributions for random input parameters $S_{0}, \beta$ and $\gamma$, which hereinafter will be assumed to be independent RVs. As the initial condition $S_{0}$ represents a percentage, it is assumed that $S_{0}$ has a Beta distribution of parameters $a>0, b>0$, i.e., $S_{0} \sim B e(a ; b)$. This is supported by the domain of a Beta $R V$, which is the interval $(0,1)$. In addition, Beta distribution is more flexible than other candidates like $(0,1)$-Uniform distribution since it is a two-parametric distribution. It permits to adapt it better to model complex situations. For the rate of decline $\beta$, an Exponential distribution of parameter $\lambda_{\beta}>0$, truncated on the interval $(0,1000)$ will be taken, because this rate must be modelled by a positive $R V$. Finally, for the rate of recovery, $\gamma$, a truncated Gaussian distribution of parameters $\mu_{\gamma}>0$ and $\sigma_{\gamma}>0$ on the interval $(0,1)$ has been chosen. This choice has been made because the positiveness of $R V \gamma$ and the great flexibility of Gaussian distribution. At this point, it is important to emphasize that the generality of the results provided in the previous section permits to choice other probability distributions for input parameters different to the aforementioned ones, whenever they match their epidemiological interpretation (positiveness, boundedness, etc). Moreover, notice that truncation for $R V s \beta$ and $\gamma$ allows to guarantee that hypotheses related to uniqueness are fulfilled.

\begin{tabular}{cccccccc}
\hline $\begin{array}{c}\text { year } \\
\left(t_{j}\right)\end{array}$ & $\begin{array}{c}1987 \\
(j=0)\end{array}$ & $\begin{array}{c}1993 \\
(j=6)\end{array}$ & $\begin{array}{c}1995 \\
(j=8)\end{array}$ & $\begin{array}{c}1997 \\
(j=10)\end{array}$ & $\begin{array}{c}2001 \\
(j=14)\end{array}$ & $\begin{array}{c}2003 \\
(j=16)\end{array}$ & $\begin{array}{c}2006 \\
(j=19)\end{array}$ \\
\hline \hline$S_{j}$ & 0.4488 & 0.5144 & 0.5278 & 0.5514 & 0.5783 & 0.6244 & 0.6467 \\
\hline
\end{tabular}

Table 4.1: Percentage of non-smokers Spanish men aged over 16 years old during the period 1987-2006. Source [68].

In order to determine positive parameters $a, b, \lambda_{\beta}, \mu_{\gamma}$ and $\sigma_{\gamma}$ associated to the probability distributions of model parameters, the mean square error, $\mathrm{E}(a, b$, 
$\left.\lambda_{\beta}, \mu_{\gamma}, \sigma_{\gamma}\right)$, between data $S_{j}$ and the expectation of $S(t)=S\left(t ; a, b, \lambda_{\beta}, \mu_{\gamma}, \sigma_{\gamma}\right)$ evaluated at the time instants $t_{j}, j \in \mathcal{J}$, will be minimized. It leads to the following optimization programme

$$
\min _{a, b, \lambda_{\beta}, \mu_{\gamma}, \sigma_{\gamma}>0} \mathrm{E}\left(a, b, \lambda_{\beta}, \mu_{\gamma}, \sigma_{\gamma}\right)=\sum_{j \in \mathcal{J}}\left(S_{j}-\mathbb{E}\left[S\left(t_{j} ; a, b, \lambda_{\beta}, \mu_{\gamma}, \sigma_{\gamma}\right)\right]\right)^{2},
$$

where, according to (4.14) and, taking into account that $S(t)=S\left(t ; a, b, \lambda_{\beta}, \mu_{\gamma}\right.$, $\left.\sigma_{\gamma}\right) \in(0,1)$, the above expectation can be computed as

$$
\mathbb{E}\left[S\left(t_{j} ; a, b, \lambda_{\beta}, \mu_{\gamma}, \sigma_{\gamma}\right)\right]=\int_{0}^{1} s f_{1}\left(s, t_{j}\right) \mathrm{d} s, \quad j \in \mathcal{J}
$$

Now, the form of the 1-PDF $f_{1}(s, t)$, that appears in the above integral and is defined by (4.11), will be explicitly, taking into account all the hypotheses that have been assumed up to now. For that, first notice that $S_{0} \sim \operatorname{Be}(a ; b)$, $\beta \sim \operatorname{Exp}\left(\lambda_{\beta}\right)$ truncated on the interval $(0,1000)$ and $\gamma \sim \mathrm{N}\left(\mu_{\gamma} ; \sigma_{\gamma}\right)$ truncated on the interval $(0,1)$, hence according to $(4.5)$, their domains $\mathcal{D}\left(S_{0}\right), \mathcal{D}(\beta)$ and $\mathcal{D}(\gamma)$ are defined by $s_{0,1}=0, s_{0,2}=1 ; \beta_{1}=0, \beta_{2}=1000$, and $\gamma_{1}=0, \gamma_{2}=1$, respectively. Therefore,

$$
\begin{aligned}
f_{1}(s, t)= & \int_{0}^{1} \int_{0}^{1000} f_{S_{0}}\left(\frac{\xi+\mathrm{e}^{(\xi-\eta) t}(-1+s) \xi-s \eta}{\xi+\mathrm{e}^{(\xi-\eta) t}(-1+s) \eta-s \eta}\right) f_{\gamma}(\xi) f_{\beta}(\eta) \\
& \times \frac{\mathrm{e}^{(\xi-\eta) t}(\xi-\eta)^{2}}{\left(\xi+\mathrm{e}^{(\xi-\eta) t}(-1+s) \eta-s \eta\right)^{2}} \mathrm{~d} \eta \mathrm{d} \xi
\end{aligned}
$$

where

$$
\begin{aligned}
f_{S_{0}}\left(\frac{\xi+\mathrm{e}^{(\xi-\eta) t}(-1+s) \xi-s \eta}{\xi+\mathrm{e}^{(\xi-\eta) t}(-1+s) \eta-s \eta}\right)= & \frac{\Gamma(a+b)}{\Gamma(a) \Gamma(b)}\left(\frac{\xi+\mathrm{e}^{(\xi-\eta) t}(-1+s) \xi-s \eta}{\xi+\mathrm{e}^{(\xi-\eta) t}(-1+s) \eta-s \eta}\right)^{a-1} \\
& \times\left(\frac{e^{(\xi-\eta) t}(-1+s)(\eta-\xi)}{\xi+\mathrm{e}^{(\xi-\eta) t}(-1+s) \eta-s \eta}\right)^{b-1}
\end{aligned}
$$


and

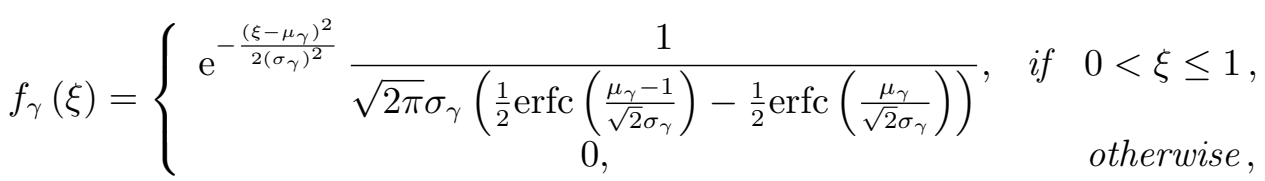

being $\operatorname{erfc}(z)=1-\frac{2}{\sqrt{\pi}} \int_{0}^{z} \mathrm{e}^{-t^{2}} \mathrm{~d} t$ the complementary error function.

In order to compute the minimum of the error function, $\mathrm{E}\left(a, b, \lambda_{\beta}, \mu_{\gamma}, \sigma_{\gamma}\right)$, given by (4.23)-(4.26), Nelder-Mead algorithm [69] carrying out computations by Mathematica ${ }^{\circledR}$ [65] has been used. The obtained results are

$$
\begin{aligned}
& a^{*}=708.755, \quad b^{*}=893.394, \quad \lambda_{\beta}^{*}=1362.230, \\
& \mu_{\gamma}^{*}=0.0231162, \quad \sigma_{\gamma}^{*}=0.0000526 .
\end{aligned}
$$

Figure 4.2 shows the 1-PDF (4.24)-(4.26) for the optimal values $a^{*}, b^{*}, \lambda_{\beta}^{*}, \mu_{\gamma}^{*}$ and $\sigma_{\gamma}^{*}$ given by (4.27). One observes that the center of the mass of probability of $R V$ percentage of susceptibles at time $t, S(t)$, rises while its dispersion reduces as time increases. This behaviour is better observed in Figure 4.3 where the mean, $\mu_{S}(t)$, and the standard deviation, $\sigma_{S}(t)$, of $S(t)$ have been depicted. The obtained results agree with the historical data from which one observes that the percentage of Spanish non-smokers men increases as time goes on.

In order to validate the model, confidence intervals have been built. As the PDF of $S(t)$ for every $t$ is known exactly, these intervals can be determined using (4.16)-(4.17). In this case, notice that their approximation using Gaussian confidence intervals of the form $\mu_{S}(t) \pm 2 \sigma_{S}(t)$ provides very similar numerical results for every year of period 1987-2006. In Figure 4.4 these intervals together with the real data are plotted.

In Table 4.2, confidence levels (CLs) for the approximate intervals $\mu_{S}(t) \pm$ $2 \sigma_{S}(t)$ have been presented using the exact PDF (4.11) for the years where data is available, although figures for the rest of the period are similar. Notice that these figures are very close to $95.45 \%$, that corresponds to the case that the PDF is Gaussian. From Figure 4.4, one observes that all data lies within the confidence intervals, hence the model is capable of capturing the variability of the real data, and, as a consequence, the model can be validated at $95 \%$ CL. 


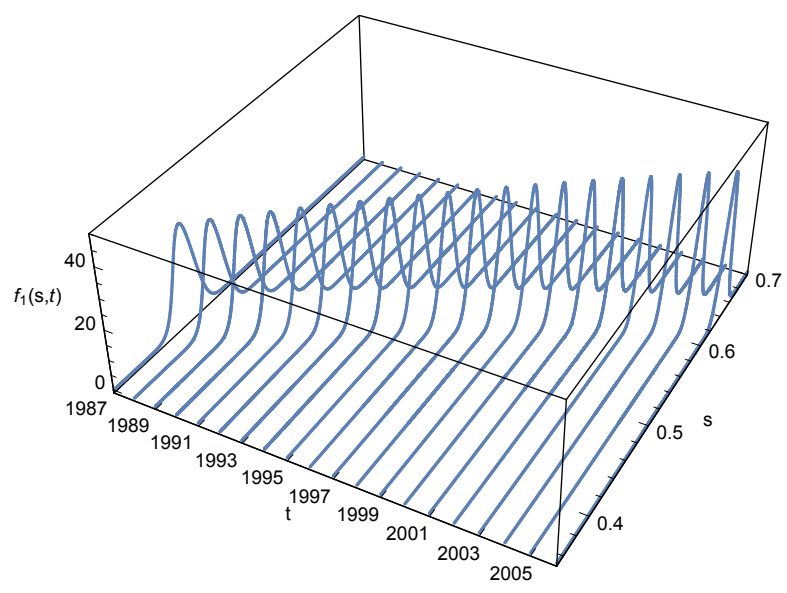

Figure 4.2: Plot of $f_{1}(s, t)$ in Example 4.1 during the period 1987-2006 (corresponding to the solid lines).

\begin{tabular}{cccccccc}
\hline $\begin{array}{c}\text { year } \\
\left(t_{j}\right)\end{array}$ & $\begin{array}{c}1987 \\
(j=0)\end{array}$ & $\begin{array}{c}1993 \\
(j=6)\end{array}$ & $\begin{array}{c}1995 \\
(j=8)\end{array}$ & $\begin{array}{c}1997 \\
(j=10)\end{array}$ & $\begin{array}{c}2001 \\
(j=14)\end{array}$ & $\begin{array}{c}2003 \\
(j=16)\end{array}$ & $\begin{array}{c}2006 \\
(j=19)\end{array}$ \\
\hline \hline CL & 0.9550 & 0.9544 & 0.9545 & 0.9546 & 0.9549 & 0.9550 & 0.9552 \\
\hline
\end{tabular}

Table 4.2: Probabilities associated to the confidence intervals built according to SIS model.

Figure 4.5 shows the 1-PDF of time $T_{S}$ until a proportion $\rho_{S}$ of population remains non-smokers (susceptible) for different values of $\rho_{S} \in\{0.45,0.50,0.55$, $0.60,0.65,0.70,0.75\}$. The plot has been performed using the following expression

$$
\begin{aligned}
f_{1}\left(t, \rho_{S}\right)= & \int_{0}^{1} \int_{0}^{1000} f_{S_{0}}\left(\frac{\xi\left(1+\mathrm{e}^{t(\xi-\eta)}\left(-1+\rho_{S}\right)\right)-\eta \rho_{S}}{\xi+\eta\left(\mathrm{e}^{t(\xi-\eta)}\left(-1+\rho_{S}\right)-\rho_{S}\right)}\right) f_{\gamma}(\xi) f_{\beta}(\eta) \\
& \times \frac{\mathrm{e}^{t(\xi-\eta)}(\xi-\eta)^{2}\left(1-\rho_{S}\right)\left|\xi-\eta \rho_{S}\right|}{\left(\xi+\eta\left(\mathrm{e}^{t(\xi-\eta)}\left(-1+\rho_{S}\right)-\rho_{S}\right)\right)^{2}} \mathrm{~d} \eta \mathrm{d} \xi
\end{aligned}
$$

derived from (4.20), taking into account that $S_{0}, \beta$ and $\gamma$ are independent $R V s$ and, that 

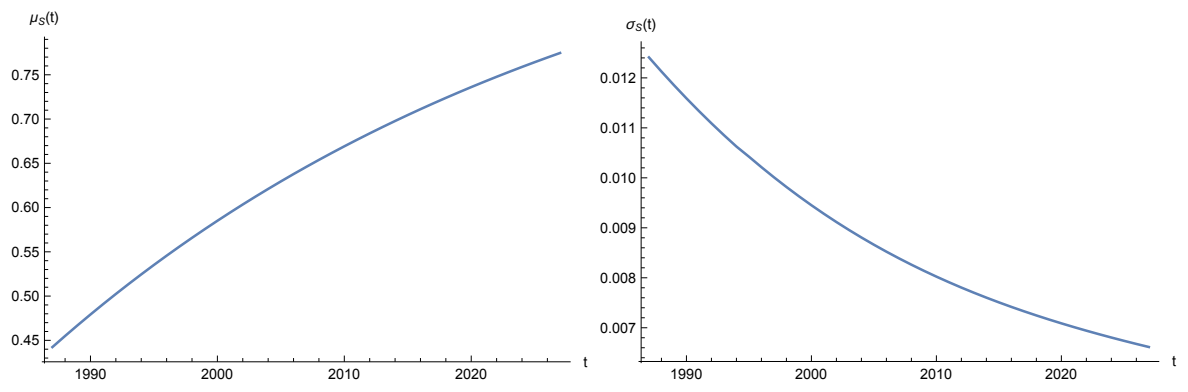

Figure 4.3: Plot of expectation function (left) and standard deviation function (right) in Example 4.1.

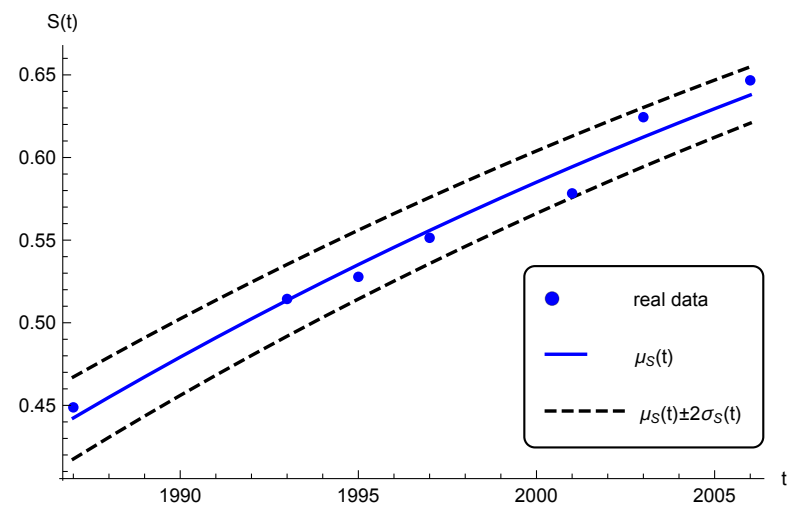

Figure 4.4: Expectation (solid line) and confidence intervals (dotted lines) in Example 4.1.

$$
\begin{aligned}
f_{S_{0}}\left(\frac{\xi\left(1+e^{t(\xi-\eta)}\left(-1+\rho_{S}\right)\right)-\eta \rho_{S}}{\xi+\eta\left(e^{t(\xi-\eta)}\left(-1+\rho_{S}\right)-\rho_{S}\right)}\right)= & \frac{\Gamma(a+b)}{\Gamma(a) \Gamma(b)}\left(\frac{\xi\left(1+e^{t(\xi-\eta)}\left(-1+\rho_{S}\right)\right)-\eta \rho_{S}}{\xi+\eta\left(e^{t(\xi-\eta)}\left(-1+\rho_{S}\right)-\rho_{S}\right)}\right)^{a-1} \\
& \times\left(\frac{(\eta-\xi) e^{t(\xi-\eta)}\left(-1+\rho_{S}\right)}{\xi+\eta\left(e^{t(\xi-\eta)}\left(-1+\rho_{S}\right)-\rho_{S}\right)}\right)^{b-1}
\end{aligned}
$$

and $f_{\beta}(\eta)$ and $f_{\gamma}(\xi)$ are given by (4.25) and (4.26), respectively. For instance, according to the 1-PDF of $T_{S}$, one gets

$$
\mathbb{E}\left[T_{S}\right]=\int_{0}^{\infty} t f_{T_{S}}(t ; 0.75) \mathrm{d} t=35.4013
$$


This means that the middle of the year 2023 approximately represents the average time until $75 \%$ of the Spanish men aged over 16 years old population will be non-smokers. This can be graphically seen in Figure 4.5. In Table 4.3, $\mathbb{E}\left[T_{S}\right]$ has been computed for different values of $\rho_{S}$. This information is crucial for health authorities in order to know the evolution of smoking and, hence adopting preventive and treatment campaigns.

\begin{tabular}{cccccccccc}
\hline$\rho_{S}$ & 0.45 & 0.50 & 0.55 & 0.60 & 0.65 & 0.70 & 0.75 & 0.80 & 0.85 \\
\hline \hline $\mathbb{E}\left[T_{S}\right]$ & 0.59 & 4.78 & 9.42 & 14.61 & 20.51 & 27.32 & 35.40 & 45.30 & 58.10 \\
\hline
\end{tabular}

Table 4.3: Expectation of time $T_{S}$ until a proportion, $\rho_{S}$, of population remains non-smoker for different values $\rho_{S}$.

Finally, using (4.22) probability of the event $\mathcal{S}$ introduced in (4.21) can be computed as

$$
\mathbb{P}[\mathcal{S}]=\int_{0}^{1} \int_{0}^{1} f_{\gamma}(\xi) f_{\beta}\left(r_{0} \xi\right)|\xi| \mathrm{d} \xi \mathrm{d} r_{0}=0.999453
$$

where $f_{\beta}(\eta)$ and $f_{\gamma}(\xi)$ are defined by (4.25) and (4.26), respectively. Notice that this result agrees with the interpretation of the basic reproductive number $R_{0}$ and, it informs that very likely the percentage of Spanish smoker men older than 16 years old will disappear as $t$ tends to $+\infty$.

\subsection{Conclusions}

In this chapter a full probabilistic description of random SIS model has been provided. The obtained results are very general in the sense that all involved input parameters have been assumed to be random variables having any probability density function. Therefore, the study includes the possibility that they are statistically either dependent or independent. In this context, significant probabilistic information has been determined. In a first step, the first probability density function of solution stochastic process of the governing non-linear differential equation has been obtained. From this crucial function, solution is completely characterized in each time instant since every statistical moment of solution can be computed. In particular, mean and variance functions are easily determined. A key point regarding the explicit determination of the first 


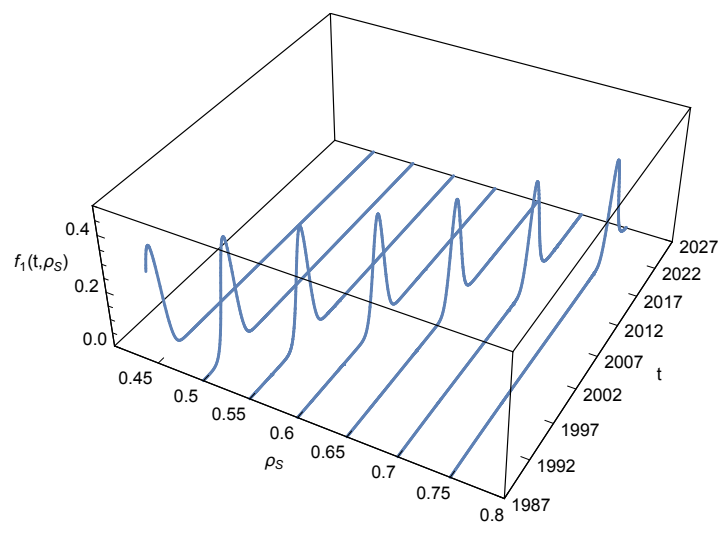

Figure 4.5: Plot of the 1-PDF of time $T_{S}$ until a proportion $\rho_{S} \in\{0.45,0.50,0.55,0.60,0.65$, $0.70,0.75\}$ (corresponding to the solid lines) of population remains susceptible in Example 4.1 .

probability density function is the computation of confidence intervals, whose confidence level is set accurately. This prevents inadvertent use of asymptotic approximations based on the Central Limit Theorem which often are difficult to justify. All the above information permits to provide a probabilistic description of SIS model that generalizes its deterministic counterpart. An important contribution of the chapter is the determination of the first probability density function of the time until a given proportion of population remains susceptible or infected. This is very useful from a practical standpoint since it permits forecasting the earliest time instant at which the susceptible subpopulation will reach a given threshold. The theoretical study has been completed by providing a stochastic interpretation of a very important parameter in Epidemiology, namely, the basic reproductive number. To put forward all the theoretical results in practice, an illustrative example using real data has been shown. The obtained results seem to be quite reliable, although it must be noticed that the choice of the probability distributions associated to random inputs is a delicate issue that constitutes itself a challenge in dealing with real problems. To improve both the theoretical study and its applications, it would be very interesting to consider a demographic model into SIS model where births and deaths rates should be included. The analysis of this more detailed model may benefit of the results developed in this chapter. Analogous comments could be applied to the extensions SIR, SEIR, etc., of SIS model. Finally it must be pointed out that alternative/complementary approaches to study these latter models can be found in $[2,27]$. 


\section{Chapter 5}

\section{Random Bernoulli differential equation}

Random Variable Transformation technique is a powerful method to determine the probabilistic solution for random differential equations represented by the first probability density function of solution stochastic process. In this chapter, that technique is applied to construct a closed form expression of solution for Bernoulli random differential equation. In order to account for the general scenario, all input parameters (coefficients and initial condition) are assumed to be absolutely continuous random variables with an arbitrary joint probability density function. The analysis is split into two cases for which an illustrative example is provided. Finally, a fish weight growth model is considered to illustrate the usefulness of the theoretical results previously established using real data. 


\subsection{Introduction}

The aim of this chapter is to continue extending the previous analysis to another important classical differential equation where probabilistic dependence among input RVs will be assumed. In the following, it will be considered the Bernoulli random IVP

$$
\left.\begin{array}{rl}
\dot{X}(t) & =C X(t)+D(X(t))^{A}, \quad t \geq t_{0}, \\
X\left(t_{0}\right) & =X_{0},
\end{array}\right\}
$$

where $t_{0}$ denotes the initial time and all input parameters, $X_{0}, D, C$ and $A$, are assumed to be absolutely continuous RVs defined in a common probability space $(\Omega, \mathcal{A}, \mathbb{P})$. Hereinafter, $\mathcal{D}\left(X_{0}\right), \mathcal{D}(D), \mathcal{D}(C)$ and $\mathcal{D}(A)$ will denote their respectively domains. In order to provide as much generality as possible throughout our analysis, hereinafter $X_{0}, C, D$ and $A$ will be assumed statistically dependent. In the following, $f_{X_{0}, D, C, A}\left(x_{0}, d, c, a\right)$ will denote their joint PDF.

The chapter is organized as follows. Section 5.2 is addressed to determine the 1-PDF of solution SP to the Bernoulli random IVP (5.1) in the general scenario where all input parameters $\left(X_{0}, D, C, A\right)$ are assumed to be RVs. As it will be shown later, this approach requires splitting the analysis in two cases. For every case, an illustrative example is also provided. In Section 5.3, ideas exhibited in Section 5.2 will be used to illustrate the usefulness of computing the 1-PDF to deal with a fish weight growth model. Conclusions are drawn in the last section.

\subsection{Probabilistic solution}

In this section, the 1-PDF, $f_{1}(x, t)$, of solution SP $X(t)$ to Bernoulli random IVP (5.1) will be determined. First, it is important to point out that the following cases have been treated in earlier contributions or in other chapters of this thesis:

- The case where coefficient $D$ is zero w.p. 1, i.e., $\mathbb{P}[\{\omega \in \Omega: D(\omega)=0\}]=$ 1 , or $A$ is one w.p. 1, i.e., $\mathbb{P}[\{\omega \in \Omega: A(\omega)=1\}]=1$, which correspond to random first-order homogeneous linear differential equation, has been studied in [16]. 
- The case where coefficient $A$ is zero w.p. 1, i.e., $\mathbb{P}[\{\omega \in \Omega: A(\omega)=$ $0\}]=1$, which corresponds to random first-order non-homogeneous linear differential equation, has been studied in [16].

- The case where coefficient $A$ is two w.p. 1, i.e., $\mathbb{P}[\{\omega \in \Omega: A(\omega)=$ $2\}]=1$, which corresponds to the Riccati random homogeneous differential equation, has been studied in Chapter 3 and [19].

Notice that a comprehensive analysis, similar to one exhibited in [16] and in Chapter 3, of all possible cases depending on deterministic and random nature of the four input parameters, $X_{0}, D, C$ and $A$, will involve 15 cases (obviously, excluding the full deterministic case where all inputs are constants). This chapter is focussed on the case where all inputs parameters are RVs with a joint PDF $f_{X_{0}, D, C, A}\left(x_{0}, d, c, a\right)$. In order to conduct the analysis of Bernoulli random IVP (5.1), it is convenient to distinguish Cases I-II listed in Table 5.1. The distinction between these two cases helps to apply RVT technique as it will apparent later (see Equation (5.4)). Hereinafter, it is assumed that

$$
\mathbb{P}[\{\omega \in \Omega: A(\omega) \neq 1\}]=1, \quad \mathbb{P}\left[\left\{\omega \in \Omega: X_{0}(\omega) \neq 0\right\}\right]=1 .
$$

Case I $\mathbb{P}[\{\omega \in \Omega: C(\omega) \neq 0\}]=1$

$$
\text { Case II } \mathbb{P}[\{\omega \in \Omega: C(\omega)=0\}]=1
$$

Table 5.1: List of the two cases considered to compute the 1-PDF of solution SP to Bernoulli random IVP (5.1) under assumptions (5.2).

Notice that in case $X_{0}=0$ w.p. $1, X(t) \equiv 0$ is clearly the unique solution of IVP (5.1).

Before presenting the study, it is important to emphasize that RVT method constitutes the unifying technique to conduct this analysis in the two cases listed in Table 5.1. With the aim of facilitating the exposition, in the subsequent subsections the results are presented following a common structure.

In each one of Cases I-II, a numerical example to illustrate the theoretical results established will be presented. In these examples statistical independence 
among inputs will be assumed to facilitate computations. In Section 5.3 a full example involving statistical dependence will be exhibited.

\subsubsection{Solving the Case I}

Let us assume that linear coefficient $C$ is different from zero w.p. 1. In the following, $f_{X_{0}, D, C, A}\left(x_{0}, d, c, a\right)$ will denote the joint PDF of random vector $\left(X_{0}, D, C, A\right)$.

In order to determine the 1-PDF of solution SP, $X(t)$, of IVP (5.1), it is convenient to consider the following change of variable

$$
X(t)=(Z(t))^{\frac{1}{1-A}} .
$$

This permits the transformation of (5.1) into the following linear IVP

$$
\left.\begin{array}{rl}
\dot{Z}(t) & =(1-A) C Z(t)+(1-A) D, \quad t \geq t_{0}, \\
Z\left(t_{0}\right) & =\left(X_{0}\right)^{1-A}
\end{array}\right\}
$$

whose exact closed form solution SP is given by

$$
Z(t)=\left(X_{0}\right)^{1-A} \mathrm{e}^{(1-A) C\left(t-t_{0}\right)}+\frac{D}{C} \mathrm{e}^{(1-A) C\left(t-t_{0}\right)}-\frac{D}{C} .
$$

Let us fix $t \geq t_{0}$ and denote $Z=Z(t)$. Let us consider the mapping $\mathbf{r}: \mathbb{R}^{4} \longrightarrow$ $\mathbb{R}^{4}$ such that

$$
\begin{aligned}
& z_{1}=r_{1}\left(x_{0}, d, c, a\right)=\left(x_{0}\right)^{1-a} \mathrm{e}^{(1-a) c\left(t-t_{0}\right)}+\frac{d}{c} \mathrm{e}^{(1-a) c\left(t-t_{0}\right)-\frac{d}{c}} \\
& z_{2}=r_{2}\left(x_{0}, d, c, a\right)=d \\
& z_{3}=r_{3}\left(x_{0}, d, c, a\right)=c \\
& z_{4}=r_{4}\left(x_{0}, d, c, a\right)=a .
\end{aligned}
$$

It is straightforward to check that the inverse mapping of $\mathbf{r}$ is defined by $\mathbf{s}: \mathbb{R}^{4} \longrightarrow \mathbb{R}^{4}$, where 


$$
\begin{aligned}
& x_{0}=s_{1}\left(z_{1}, z_{2}, z_{3}, z_{4}\right)=\left(\frac{-z_{2}+\mathrm{e}^{-z_{3}\left(t-t_{0}\right)\left(1-z_{4}\right)}\left(z_{1} z_{3}+z_{2}\right)}{z_{3}}\right)^{\frac{1}{1-z_{4}}}, \\
& d=s_{2}\left(z_{1}, z_{2}, z_{3}, z_{4}\right)=z_{2}, \\
& c=s_{3}\left(z_{1}, z_{2}, z_{3}, z_{4}\right)=z_{3}, \\
& a=s_{4}\left(z_{1}, z_{2}, z_{3}, z_{4}\right)=z_{4} .
\end{aligned}
$$

Moreover, the Jacobian of mapping $\mathbf{s}$ is given by

$$
J_{4}=\frac{\mathrm{e}^{-z_{3}\left(t-t_{0}\right)\left(1-z_{4}\right)}}{1-z_{4}}\left(\frac{-z_{2}+\mathrm{e}^{-z_{3}\left(t-t_{0}\right)\left(1-z_{4}\right)}\left(z_{1} z_{3}+z_{2}\right)}{z_{3}}\right)^{\frac{z_{4}}{1-z_{4}}} \neq 0 .
$$

Therefore, by Theorem 2.1, the joint $\mathrm{PDF}$ of random vector $\mathbf{Z}=\left(Z_{1}, Z_{2}, Z_{3}, Z_{4}\right)$ can be written as

$$
\begin{aligned}
f_{\mathbf{Z}}\left(z_{1}, z_{2}, z_{3}, z_{4}\right)= & f_{X_{0}, D, C, A}\left(\left(\frac{-z_{2}+\mathrm{e}^{-z_{3}\left(t-t_{0}\right)\left(1-z_{4}\right)}\left(z_{1} z_{3}+z_{2}\right)}{z_{3}}\right)^{\frac{1}{1-z_{4}}}, z_{2}, z_{3}, z_{4}\right) \\
& \times\left|\frac{\mathrm{e}^{-z_{3}\left(t-t_{0}\right)\left(1-z_{4}\right)}}{1-z_{4}}\left(\frac{-z_{2}+\mathrm{e}^{-z_{3}\left(t-t_{0}\right)\left(1-z_{4}\right)}\left(z_{1} z_{3}+z_{2}\right)}{z_{3}}\right)^{\frac{z_{4}}{1-z_{4}}}\right|
\end{aligned}
$$

Taking into account (5.3), $Z=Z_{1}, A=Z_{4}$, hence the PDF of random vector $(Z, A)$ is obtained as the marginal PDF of $f_{Z_{1}, Z_{2}, Z_{3}, Z_{4}}\left(z_{1}, z_{2}, z_{3}, z_{4}\right)$ with respect to $Z_{2}=D$ and $Z_{3}=C$, that is,

$$
\begin{aligned}
f_{Z, A}(z, a)= & \iint_{\mathcal{D}(D, C)} f_{X_{0}, D, C, A}\left(\left(\frac{-d+\mathrm{e}^{-c\left(t-t_{0}\right)(1-a)}(z c+d)}{c}\right)^{\frac{1}{1-a}}, d, c, a\right) \\
& \times\left|\frac{\mathrm{e}^{-c\left(t-t_{0}\right)(1-a)}}{1-a}\left(\frac{-d+\mathrm{e}^{-c\left(t-t_{0}\right)(1-a)}(z c+d)}{c}\right)^{\frac{a}{1-a}}\right| \mathrm{d} c \mathrm{~d} d .
\end{aligned}
$$


Now, using the change of variable (5.3), applying Proposition 2.8 with the following identification: $U_{1}=Z, U_{2}=A$ and $W=X$, and considering $t \geq t_{0}$ arbitrary, the 1-PDF of solution SP $X(t)=(Z(t))^{\frac{1}{1-A}}$ is given by

$$
\begin{aligned}
f_{1}(x, t)= & \iiint_{\mathcal{D}(A, D, C)} f_{X_{0}, D, C, A}\left(\left(\frac{-d+\mathrm{e}^{-c\left(t-t_{0}\right)(1-a)}\left(x^{1-a} c+d\right)}{c}\right)^{\frac{1}{1-a}}, d, c, a\right) \\
& \times\left|\frac{\mathrm{e}^{-c\left(t-t_{0}\right)(1-a)}}{x^{a}}\left(\frac{-d+\mathrm{e}^{-c\left(t-t_{0}\right)(1-a)}\left(x^{1-a} c+d\right)}{c}\right)^{\frac{a}{1-a}}\right| \mathrm{d} c \mathrm{~d} d \mathrm{~d} a .
\end{aligned}
$$

Example 5.1 Let us consider random IVP (5.1) with $t_{0}=0$ and assume the following probability distributions for its inputs parameters: nonlinear coeffcient, $D$, is a Beta $R V$ with parameters $(2,3), D \sim \operatorname{Be}(2 ; 3)$; linear coeffcient, $C$, is an Exponential $R V$ of mean $1 / 35, C \sim \operatorname{Exp}(35)$; exponent $A$ is an Uniform $R V$ on the interval $[0,1], A \sim \mathrm{U}([0,1])$, and initial condition is an Exponential $R V$ with mean $2.5, X_{0} \sim \operatorname{Exp}(1 / 2.5)$.

In Figure 5.1, the 1-PDF $f_{1}(x, t)$ of solution SP to random IVP (5.1) has been plotted. Computations to perform this graphical representation have been carried out using expression (5.6) on time interval [0,5]. Notice that the joint $P D F$ of input data $\left(X_{0}, D, C, A\right)$ is given by

$$
\begin{aligned}
f_{X_{0}, D, C, A}\left(x_{0}, d, c, a\right) & =f_{X_{0}}\left(x_{0}\right) f_{D}(d) f_{C}(c) f_{A}(a) \\
& =\left(\frac{1}{2.5} \mathrm{e}^{-\frac{1}{2.5} x_{0}}\right) \times(1) \times\left(35 \mathrm{e}^{-35 c}\right) \times\left(12 d(1-d)^{2}\right) \\
& =168 \mathrm{e}^{-\left(0.4 x_{0}+35 c\right)} d(1-d)^{2},
\end{aligned}
$$

if $\left(x_{0}, d, c, a\right) \in \mathcal{D}\left(X_{0}\right) \times \mathcal{D}(D) \times \mathcal{D}(C) \times \mathcal{D}(A)$, being $\left.\mathcal{D}\left(X_{0}\right)=\mathcal{D}(C)=\right] 0,+\infty[$, $\mathcal{D}(D)=\mathcal{D}(A)=] 0,1\left[\right.$, and $f_{X_{0}, D, C, A}\left(x_{0}, d, c, a\right)=0$ otherwise. Notice that hypothesis of pairwise independence of $R V s X_{0}, A, C$ and $D$ has been used in the above expression for $f_{X_{0}, D, C, A}\left(x_{0}, d, c, a\right)$. From a computational point of view it is worth pointing out that graphical representation shown in Figure 5.1 has been built fixing a value of time $t=\hat{t} \in\{0,0.5,1,1.5,2, \ldots, 5\}$ and then, first checking that $\int_{-\infty}^{\infty} f_{1}(x, \hat{t}) \mathrm{d} x \approx 1$, and secondly, using the Mathematica ${ }^{\circledR}$ software, [65] to calculate the three-dimensional integral given by (5.6). 
From this graph one observes that the variability of solution $X=X(t)$ increases rapidly over the time.

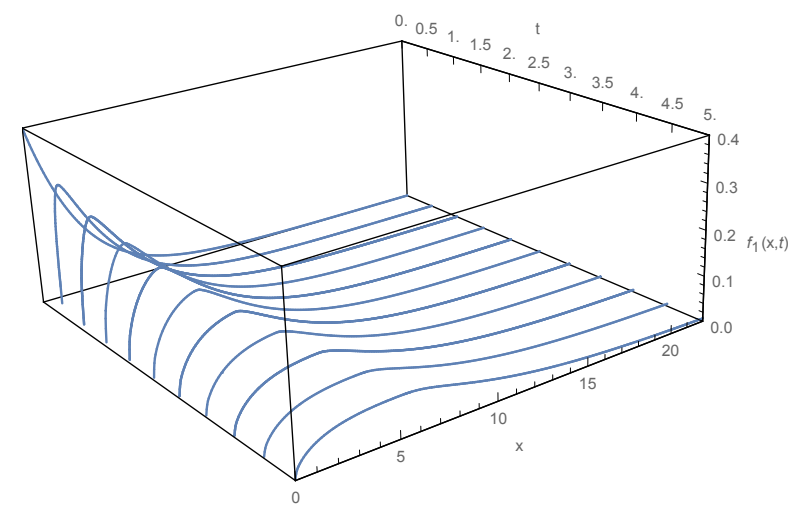

Figure 5.1: Plot of the 1-PDF $f_{1}(x, t)$ given by (5.6) in Example 5.1 at values of $t \in\{0,0.5$, $1,1.5, \ldots, 5\}$ (corresponding to the solid lines).

\subsubsection{Solving the Case II}

Now, the case where $C$ is 0 w.p. 1 will be considered. This corresponds to the following particular case of IVP (5.1)

$$
\left.\begin{array}{rl}
\dot{X}(t) & =D(X(t))^{A}, \quad t \geq t_{0} \\
X\left(t_{0}\right) & =X_{0}
\end{array}\right\}
$$

whose solution is given by

$$
X(t)=\left(D\left(t-t_{0}\right)(1-A)+\left(X_{0}\right)^{1-A}\right)^{\frac{1}{1-A}} .
$$

Let us fix $t \geq t_{0}$. Unlike Case I, now transformation (5.3), involving $\operatorname{RV~} Z$, is not needed to compute solution of IVP (5.7). Hence, the 1-PDF of $X(t)$ will be computed directly without computing previously the PDF of RV $Z$. With this end, let us define the mapping $\mathbf{r}: \mathbb{R}^{3} \longrightarrow \mathbb{R}^{3}$ such that 


$$
\begin{aligned}
& x_{1}=r_{1}\left(x_{0}, d, a\right)=\left(d\left(t-t_{0}\right)(1-a)+\left(x_{0}\right)^{1-a}\right)^{\frac{1}{1-a}}, \\
& x_{2}=r_{2}\left(x_{0}, d, a\right)=d \\
& x_{3}=r_{3}\left(x_{0}, d, a\right)=a .
\end{aligned}
$$

The inverse mapping $\mathbf{s}$ of $\mathbf{r}$ is given by

$$
\begin{aligned}
& x_{0}=s_{1}\left(x_{1}, x_{2}, x_{3}\right)=\left(\left(x_{1}\right)^{1-x_{3}}-x_{2}\left(t-t_{0}\right)+x_{2} x_{3}\left(t-t_{0}\right)\right)^{\frac{1}{1-x_{3}}} \\
& d=s_{2}\left(x_{1}, x_{2}, x_{3}\right)=x_{2} \\
& a=s_{3}\left(x_{1}, x_{2}, x_{3}\right)=x_{3} .
\end{aligned}
$$

In accordance with RVT method, Theorem 2.1, the joint PDF of random vector $\mathbf{X}=\left(X_{1}, X_{2}, X_{3}\right)$ is given by

$$
\begin{aligned}
f_{\mathbf{X}}\left(x_{1}, x_{2}, x_{3}\right)= & f_{X_{0}, D, A}\left(\left(\left(x_{1}\right)^{1-x_{3}}-x_{2}\left(t-t_{0}\right)+x_{2} x_{3}\left(t-t_{0}\right)\right)^{\frac{1}{1-x_{3}}}, x_{2}, x_{3}\right) \\
& \times\left|\left(x_{1}\right)^{-x_{3}}\left(\left(x_{1}\right)^{1-x_{3}}-x_{2}\left(t-t_{0}\right)+x_{2} x_{3}\left(t-t_{0}\right)\right)^{\frac{x_{3}}{1-x_{3}}}\right|,
\end{aligned}
$$

where the factor in absolute value corresponds to the Jacobian of mapping $\mathbf{s}$.

Finally, considering $t \geq t_{0}$ arbitrary, the 1-PDF of solution $\mathrm{SP} X(t)$ to $\operatorname{IVP}(5.7)$ is obtained as the $\left(X_{2}, X_{3}\right)$-marginal of the PDF (5.8)

$$
\begin{aligned}
f_{1}(x, t)= & \iint_{\mathcal{D}(D, A)} f_{X_{0}, D, A}\left(\left(x^{1-a}-d\left(t-t_{0}\right)+a d\left(t-t_{0}\right)\right)^{\frac{1}{1-a}}, d, a\right) \\
& \times\left|x^{-a}\left(x^{1-a}-d\left(t-t_{0}\right)+a d\left(t-t_{0}\right)\right)^{\frac{a}{1-a}}\right| \mathrm{d} a \mathrm{~d} d .
\end{aligned}
$$

Remark 5.1 Notice that expression (5.9) is not a particular case of expression (5.6) since in the context of the Case II it is assumed that $C=0$ w.p. 1. Revising carefully the application of Theorem 2.16 within our discussion of Case I, the hypothesis $C=0$ w.p. 1 is required to guarantee $J_{3} \neq 0$ (see expression (5.5) where $z_{3}=c$ ). Moreover, expression (5.9) cannot be obtained as a limit of expression (5.6) as $c \rightarrow 0$. These facts have motivated the distinction of the two cases listed in Table 5.1. 
Example 5.2 In order to illustrate the theoretical results previously established, let us consider random IVP (5.7) being initial condition $X_{0}$ an Exponential $R V$ with mean $1, X_{0} \sim \operatorname{Exp}(1)$; non-linear coefficient $D$ a Beta $R V$ $D \sim \operatorname{Be}(2 ; 3)$ and exponent $A$ a standard Gaussian $R V, A \sim \mathrm{N}(0 ; 1)$, truncated at domain $]-\infty, 1]$. Figure 5.2 shows the 1-PDF of solution SP to IVP (5.7) on the time interval $[0,6]$. It has been computed according to expression (5.9) and taking into account that

$$
\begin{aligned}
f_{X_{0}, D, A}\left(x_{0}, d, a\right) & =f_{X_{0}}\left(x_{0}\right) f_{D}(d) f_{A}(a) \\
& =\left(\mathrm{e}^{-x_{0}}\right) \times\left(12 d(1-d)^{2}\right) \times \frac{\mathrm{e}^{-\frac{a^{2}}{2}}}{\int_{-\infty}^{1} \mathrm{e}^{-\frac{z^{2}}{2}} \mathrm{~d} z} \\
& =\sqrt{\frac{2}{\pi}} \frac{12}{1+\operatorname{erfc}\left(\frac{\sqrt{2}}{2}\right)} \mathrm{e}^{-\left(x_{0}+\frac{a^{2}}{2}\right)} d(1-d)^{2},
\end{aligned}
$$

if $\left(x_{0}, d, a\right) \in \mathcal{D}\left(X_{0}\right) \times \mathcal{D}(D) \times \mathcal{D}(A)$, being $\left.\mathcal{D}\left(X_{0}\right)=\right] 0,+\infty[, \mathcal{D}(D)=] 0,1[$, $\mathcal{D}(A)=]-\infty, 1\left[\right.$, and $f_{X_{0}, D, A}\left(x_{0}, d, a\right)=0$ otherwise. Here, expression $\operatorname{erfc}(t)=$ $\frac{2}{\sqrt{\pi}} \int_{0}^{t} \mathrm{e}^{-z^{2}} \mathrm{~d} z$ stands for the error function. Similar comments to the ones exhibited in Example 5.1 regarding computations carried out using Mathematica ${ }^{\circledR}$ software to plot the 1-PDF $f_{1}(x, t)$ can be made. From this representation, one observes that the variance increases as time goes on.

\subsection{An application of Bernoulli random differential equation to modelling}

On the one hand, so far closed expressions for the 1-PDF of solution SP to Bernoulli random IVP (5.1) have been provided for the two cases listed in Table 5.1. On the other hand, as it has been highlighted in the Introduction (Chapter 1), random differential equations are very useful in modelling. Next, this claim will be illustrated by considering a deterministic model, formulated by a Bernoulli differential equation, that describes the fish weight growth over the time. In a first step, to assume that input parameters (coefficients and initial condition) are RVs rather than deterministic constants. Secondly, using real data to assign a reliable probabilistic distribution to random inputs using an inverse frequentist technique. Then, to take advantage of RVT technique to determine the 1-PDF of solution SP to the model. Finally, from this important 


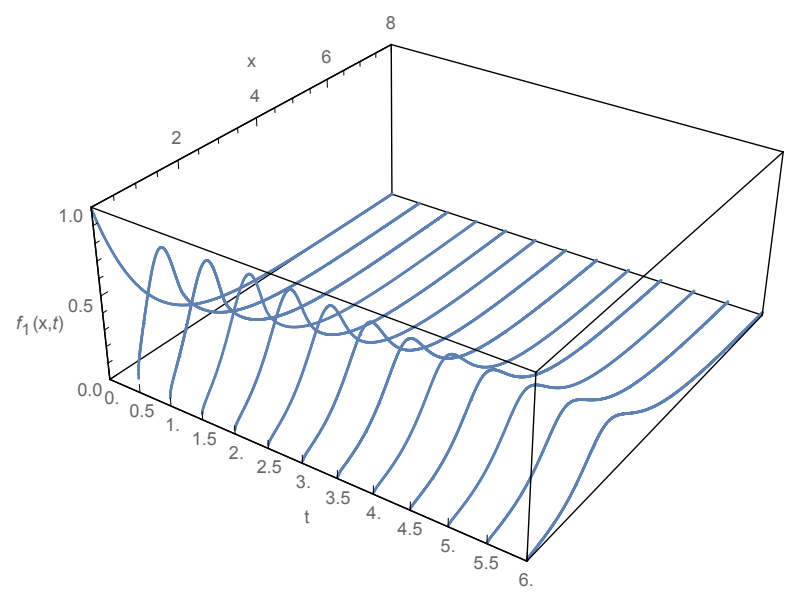

Figure 5.2: Plot of the 1-PDF $f_{1}(x, t)$ given by (5.9) in Example 5.2 at the values of $t \in\{0,0.5 .1,1.5, \ldots, 6\}$ (corresponding to the solid lines).

deterministic function, both punctual and probabilistic predictions based on confidence intervals will be constructed.

The following IVP, usually referred to as Bertalanffy model $[8,9]$, has been applied extensively to describe the fish weight growth, $W(t)$, at time instant $t$, (see for example, $[10,67]$ )

$$
\left.\begin{array}{rl}
\dot{W}(t) & =-\lambda W(t)+\eta(W(t))^{2 / 3}, \quad t \geq t_{0}, \\
W\left(t_{0}\right) & =W_{0} .
\end{array}\right\}
$$

It is worth to point out that, recently, it have been considered stochastic versions of this model based on Itô-type stochastic differential equations, [75, 81].

Under the approach of the chapter, let us assume that initial condition, $W_{0}$, and coefficients, $\eta$ and $\lambda$ of IVP (5.10) are absolutely continuous RVs defined on a common probability space $(\Omega, \mathcal{A}, \mathbb{P})$, being $f_{W_{0}, \eta, \lambda}\left(w_{0}, \eta, \lambda\right)$ their joint PDF. Moreover, let us suppose that

$$
\mathbb{P}\left[\left\{\omega \in \Omega: W_{0}(\omega) \neq 0\right\}\right]=1, \quad \mathbb{P}[\{\omega \in \Omega: \lambda(\omega) \neq 0\}]=1 .
$$

Considering identification $X(t)=W(t), C=-\lambda, D=\eta, A=2 / 3$ and $X_{0}=W_{0}$, IVP (5.10) can be treated as a particular case of (5.1). Hence, one 
can obtain the 1-PDF $f_{1}(w, t)$ of solution SP $W(t)$ of (5.10) introducing Dirac delta function, $\delta(a-2 / 3)$, in expression (5.6). An alternative way to do that is to treat problem (5.10) directly depending on the three $\operatorname{RVs} W_{0}, \eta, \lambda$, in a similar way as in the Case I. With this aim, consider the change of variable

$$
W(t)=(Z(t))^{3}
$$

This permits the transformation of (5.10) into the following random linear IVP

$$
\left.\begin{array}{l}
\dot{Z}(t)=-(1 / 3) \lambda Z(t)+(1 / 3) \eta, \quad t \geq t_{0}, \\
Z\left(t_{0}\right)=\left(W_{0}\right)^{1 / 3},
\end{array}\right\}
$$

whose solution SP is given by

$$
Z(t)=\left(W_{0}\right)^{1 / 3} \mathrm{e}^{-(1 / 3) \lambda\left(t-t_{0}\right)}-\frac{\eta}{\lambda} \mathrm{e}^{-(1 / 3) \lambda\left(t-t_{0}\right)}+\frac{\eta}{\lambda}
$$

Following an analogous reasoning exhibited in the Case I, based on to apply RVT technique, the PDF of $\operatorname{RV} Z=Z(t)$ is obtained with $t \geq t_{0}$ fixed,

$$
\begin{aligned}
f_{Z}(z)= & 3 \iint_{\mathcal{D}(\eta, \lambda)} f_{W_{0}, \eta, \lambda}\left(\left(\frac{\mathrm{e}^{(1 / 3) \lambda\left(t-t_{0}\right)} \lambda z+\eta-\mathrm{e}^{(1 / 3) \lambda\left(t-t_{0}\right)} \eta}{\lambda}\right)^{3}, \eta, \lambda\right) \\
& \times\left(\frac{\mathrm{e}^{(1 / 3) \lambda\left(t-t_{0}\right)} \lambda z+\eta-\mathrm{e}^{(1 / 3) \lambda\left(t-t_{0}\right)} \eta}{\lambda}\right)^{2} \mathrm{e}^{(1 / 3) \lambda\left(t-t_{0}\right)} \mathrm{d} \lambda \mathrm{d} \eta
\end{aligned}
$$

Now, using the change of variable (5.11), for every $t \geq t_{0}$, the 1-PDF of solution SP $W=W(t)$ that represents the fish weight growth is obtained applying Proposition 2.9

$$
\begin{aligned}
f_{1}(w, t)= & \frac{1}{3} f_{Z}\left(w^{1 / 3}\right)|w|^{-2 / 3} \\
= & \iint_{\mathcal{D}(\eta, \lambda)} f_{W_{0}, \eta, \lambda}\left(\left(\frac{\mathrm{e}^{(1 / 3) \lambda\left(t-t_{0}\right)} \lambda w^{1 / 3}+\eta-\mathrm{e}^{(1 / 3) \lambda\left(t-t_{0}\right)} \eta}{\lambda}\right)^{3}, \eta, \lambda\right) \\
& \times\left(\frac{\mathrm{e}^{(1 / 3) \lambda\left(t-t_{0}\right)} \lambda w^{1 / 3}+\eta-\mathrm{e}^{(1 / 3) \lambda\left(t-t_{0}\right)} \eta}{\lambda}\right)^{2} \mathrm{e}^{(1 / 3) \lambda\left(t-t_{0}\right)}|w|^{-2 / 3} \mathrm{~d} \lambda \mathrm{d} \eta .
\end{aligned}
$$


Table 5.2 shows data about fish weights in lbs for walleye species over the period 1-33 in years [94]. Notice that the sample size is $N=33$.

\begin{tabular}{cccccccccccc}
\hline$t_{i}$ (years) & 1 & 2 & 3 & 4 & 5 & 6 & 7 & 8 & 9 & 10 & 11 \\
\hline$w_{i}$ (lbs) & 0.2 & 0.4 & 0.6 & 0.9 & 1 & 1.3 & 1.6 & 1.8 & 2.3 & 2.6 & 2.9 \\
\hline \hline$t_{i}$ (years) & 12 & 13 & 14 & 15 & 16 & 17 & 18 & 19 & 20 & 21 & 22 \\
\hline$w_{i}(\mathrm{lbs})$ & 3.1 & 3.4 & 3.7 & 4.5 & 5.2 & 5.7 & 6.2 & 6.5 & 6.7 & 6.8 & 7.2 \\
\hline \hline$t_{i}$ (years) & 23 & 24 & 25 & 26 & 27 & 28 & 29 & 30 & 31 & 32 & 33 \\
\hline$w_{i}(\mathrm{lbs})$ & 8.2 & 9 & 9.5 & 10 & 10.5 & 11 & 11.5 & 12 & 12.5 & 13 & 14 \\
\hline
\end{tabular}

Table 5.2: Fish weights $w_{i}$ for walleye species in lbs every year $t_{i}, 1 \leq i \leq 33=N$, [94].

Next, random Bertalanffy model (5.10) with $t_{0}=1$ will be applied to describe the evolution of fish weight over the time. For that purpose, first it shall be assigned a reliable probabilistic distribution to input random vector $\mathbf{Q}=\left(W_{0}, \eta, \lambda\right)$. To this end, several methods and techniques are available such as frequentist and bayessian techniques [5, 83, 87]. Moreover, the analysis can be complemented using parameter identifications techniques that may improve results ([83, Chapter 6],[56]). In this chapter, this will be done applying an inverse frequentist technique for parameter estimation, which is a particular Inverse Uncertainty Quantification technique. As the results established in Section 5.2 are valid for dependent RVs, and accounting for statistical dependence among input parameters $\left(W_{0}, \eta, \lambda\right)$ is an important issue, an inverse frequentist approach has been chosen because it allows to consider statistical dependence in a very flexible manner. Under inverse frequentist technique, it is assumed that the measured quantity of interest, i.e., fish weights $w_{i}$ of observations $\left(t_{i}, w_{i}\right), 1 \leq i \leq N=33$ are corrupted by measurement errors $\epsilon_{i}$, i.e.,

$$
w_{i}=W\left(t_{i} ; \mathbf{q}\right)=W\left(t_{i} ; w_{0}, \eta, \lambda\right)+\epsilon_{i}, \quad 1 \leq i \leq 33=N
$$

As usual, notice that lower-case letters is used to emphasize that the model is being evaluated at specific numerical values $\left(t_{i}, w_{i}\right)$. 
The mathematical inverse problem associated with parameter estimation can then be stated as follows: to quantify uncertainty associated with $\mathbf{q}=\left(w_{0}, \eta, \lambda\right)$ from the measurement errors $\epsilon_{i}$, and then assigning a probabilistic distribution to random vector $\mathbf{Q}=\left(W_{0}, \eta, \lambda\right)$. A basic tenet of inverse frequentist approach, that will be checked later, is that errors are i.i.d. and $\epsilon_{i} \sim \mathrm{N}\left(0 ; \sigma^{2}\right)$, being $\sigma>0$ fixed but unknown. As a consequence of this assignment of uncertainty, to model parameters through measurement errors according to the formulation (5.14), the probabilistic distribution for random vector $\mathbf{Q}=\left(W_{0}, \eta, \lambda\right)$ is assumed to be a multivariate Gaussian distribution

$$
\mathbf{Q}=\left(W_{0}, \eta, \lambda\right) \sim \mathrm{N}_{3}\left(\mu_{\mathbf{Q}} ; \Sigma_{\mathbf{Q}}\right),
$$

where the mean vector $\mu_{\mathbf{Q}}=\left(\hat{w}_{0}, \hat{\eta}, \hat{\lambda}\right)$ is defined from appropriate estimates of $\left(w_{0}, \eta, \lambda\right)$ and $\Sigma_{\mathbf{Q}}$ represents the variance-covariance matrix that will be determined below.

In order to achieve this goal, first notice that from (5.11) and (5.12), it is clear that dependence of weight $W(t ; \mathbf{q})$ when $\mathbf{q}$ is nonlinear. A least squares fit to data yields the following parameter estimates

$$
\mu_{\mathbf{Q}}=\left(\hat{w}_{0}, \hat{\eta}, \hat{\lambda}\right), \quad \hat{w}_{0}=0.365934, \quad \hat{\eta}=0.305461, \quad \hat{\lambda}=0.0880184 .
$$

The residuals of the fitting are,

$$
\epsilon_{i}=W\left(t_{i} ; \hat{w}_{0}, \hat{\eta}, \hat{\lambda}\right)-w_{i}, \quad 1 \leq i \leq 33=N
$$

where, $W\left(t_{i} ; \hat{w}_{0}, \hat{\eta}, \hat{\lambda}\right)$ is solution of IVP (5.10) with $t_{0}=1$ evaluated at every year $t_{i}=i \in\{1,2, \ldots, 33\}$ with model parameters given by (5.15) and, $w_{i}$ the fish weight data collected in Table 5.2. Notice that $W\left(t ; \hat{w}_{0}, \hat{\eta}, \hat{\lambda}\right)=W(t)$ is obtained from (5.11) and (5.12). Model fitting and residuals are shown in Figure 5.3 (left) and Figure 5.3 (right), respectively. From this latter graphical representation, one observes that residuals do not exhibit discernible pattern, thus motivating the assumption that errors are i.i.d. To check that errors are normally distributed (null hypothesis), a Shapiro-Walk test has been applied. Fixed a confidence level, say $\alpha$, null hypothesis is rejected when the $p$-value is smaller than $\alpha$; otherwise the normality of the residuals is accepted (i.e., cannot be rejected) [82]. Here, Shapiro-Walk test has been applied taking $\alpha=0.05$, showing the normality of residuals (see Table 5.3). This conclusion has been reinforced by means a Q-Q plot (see Figure 5.4). 


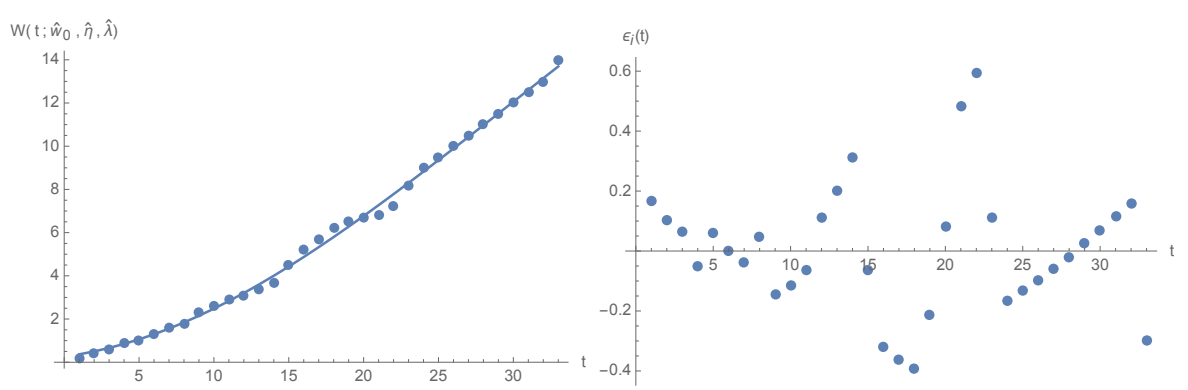

Figure 5.3: Left: Model fitting to fish weights using least mean squares method. Right: Residuals at the $N=33$ fish weights data.

\begin{tabular}{ccc}
\hline Normality Test & Statistic & $p$-value \\
\hline \hline Shapiro-Walk Test & 0.958995 & 0.242077 \\
\hline
\end{tabular}

Table 5.3: Shapiro-Walk test to check normality of residuals.

From the least mean square fitting, the error standard deviation estimate is given by

$$
\sigma=\sqrt{\frac{1}{N-p} \sum_{i=1}^{N}\left(\epsilon_{i}\right)^{2}}=0.214435, \quad \text { with } N=33, p=3,
$$

where the residuals $\epsilon_{i}$, are defined by (5.16). 


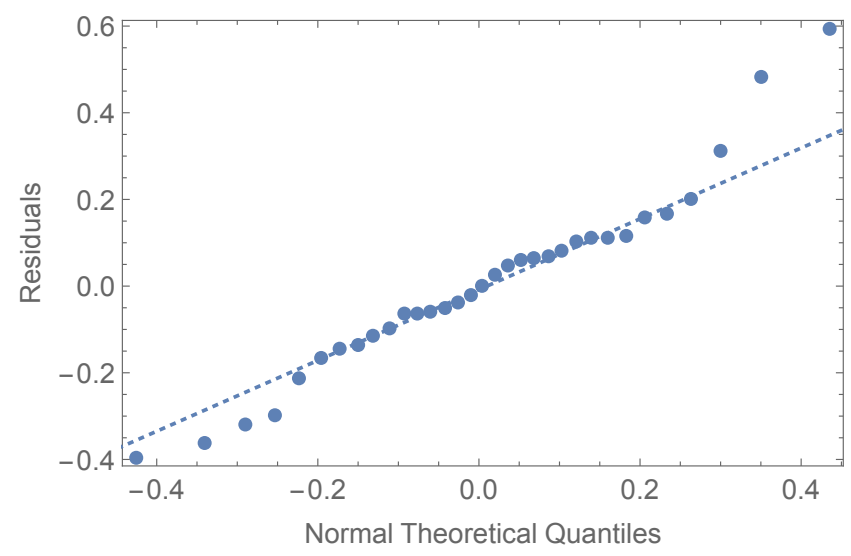

Figure 5.4: Q-Q plot for the least mean squares residuals.

According to frequentist parameter estimation method described in [83], to account for variability of the $p=3$ model parameters, $\mathbf{Q}=\left(W_{0}, \eta, \lambda\right)$, the $N \times p=33 \times 3$ sensitivity matrix

$$
\chi(\mathbf{Q})=\left[\left.\begin{array}{ccc}
\frac{\partial W\left(t_{1} ; \mathbf{Q}\right)}{\partial W_{0}} & \cdots & \frac{\partial W\left(t_{33} ; \mathbf{Q}\right)}{\partial W_{0}} \\
\frac{\partial W\left(t_{1} ; \mathbf{Q}\right)}{\partial \eta} & \cdots & \frac{\partial W\left(t_{33} ; \mathbf{Q}\right)}{\partial \eta} \\
\frac{\partial W\left(t_{1} ; \mathbf{Q}\right)}{\partial \lambda} & \cdots & \frac{\partial W\left(t_{33} ; \mathbf{Q}\right)}{\partial \lambda}
\end{array}\right|_{\mathbf{Q}=\left(\hat{w}_{0}, \hat{\eta}, \hat{\lambda}\right)}\right.
$$

has been computed. A graphical representation of entries of this matrix by columns has been plotted in Figure 5.5. From these representations, and taking into account their vertical scales, one deduces that the critical model input parameter with respect to the sensitivity analysis is $\lambda$. 


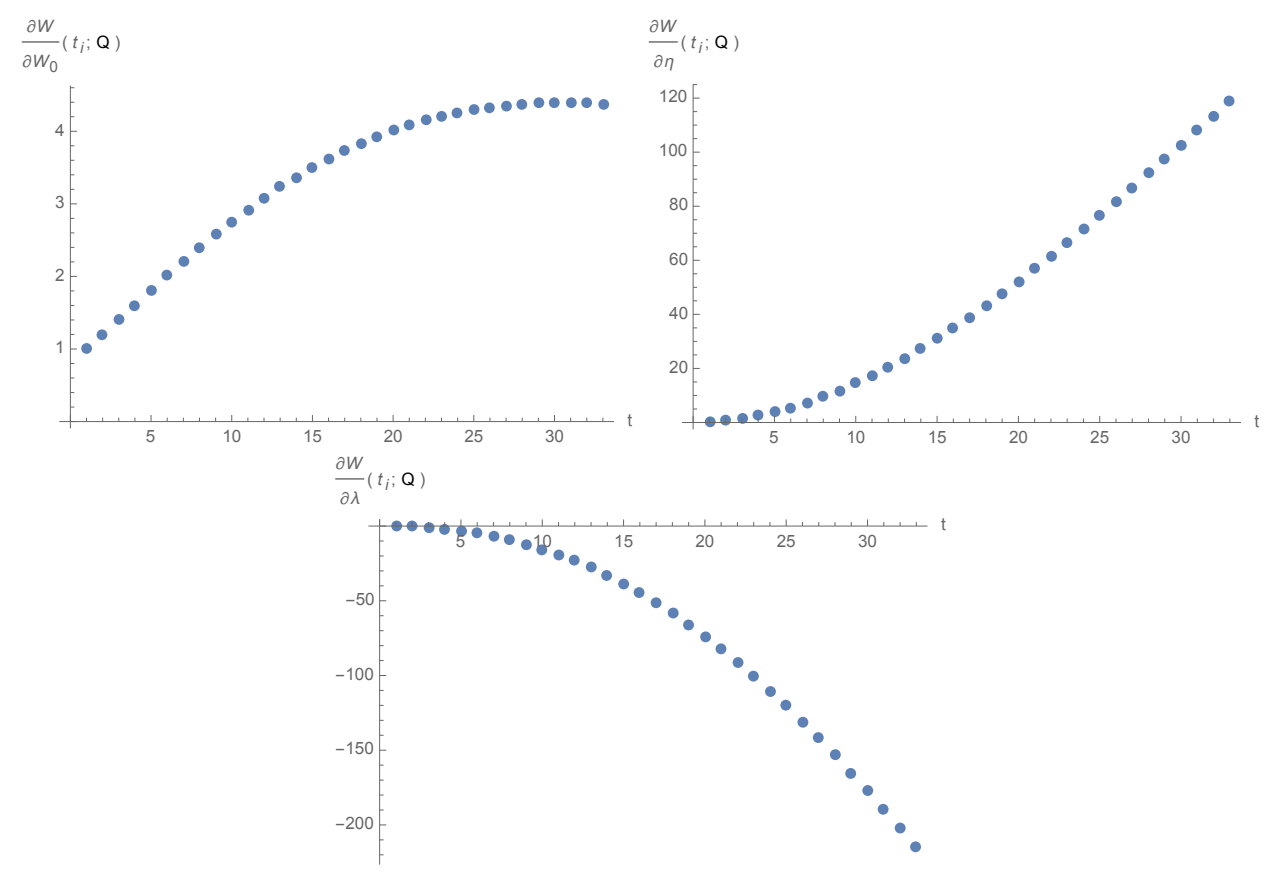

Figure 5.5: Analytic sensibility values of matrix (5.18). Left: $\frac{\partial W}{\partial W_{0}}\left(t_{i} ; \mathbf{Q}\right) . \quad$ Right: $\frac{\partial W}{\partial \eta}\left(t_{i} ; \mathbf{Q}\right)$. Center: $\frac{\partial W}{\partial \lambda}\left(t_{i} ; \mathbf{Q}\right) \cdot t_{i}=i, 1 \leq i \leq 33=N$.

Then, from (5.17) and (5.18), one obtains the covariance matrix of model parameters

$$
\begin{aligned}
\Sigma_{\mathbf{Q}}= & \sigma^{2}\left((\chi(\mathbf{Q}))^{\top} \chi(\mathbf{Q})\right)^{-1} \\
= & {\left[\begin{array}{ccc}
0.0029288 & -0.0008123 & -0.0004003 \\
-0.0008123 & 0.0002681 & 0.0001369 \\
-0.0004003 & 0.0001369 & 0.0000705
\end{array}\right] . }
\end{aligned}
$$

It is worth nothing that $\lambda$ parameter has the smallest variability $(0.0000705)$. This is in agreement with the fact that it is the most critical parameter regarding the sensibility of the model. 
Summarizing, based on inverse frequentist technique for parameter estimation approach the following probabilistic distribution has been assigned to model parameters

$$
\mathbf{Q}=\left(W_{0}, \eta, \lambda\right) \sim \mathrm{N}_{3}\left(\mu_{\mathbf{Q}} ; \Sigma_{\mathbf{Q}}\right)
$$

where $\mu_{\mathbf{Q}}$ and $\Sigma_{\mathbf{Q}}$ are given by (5.15) and (5.19), respectively.

At this point, the theoretical results previously established about the 1-PDF of solution SP to random Bertalanffy model will be considered. In Figure 5.6 (left), the 1-PDF is plotted for every time of the whole sample. As the 1-PDF is leptokurtic and it has little variance, it has been plotted only for values of $w$ where it is greater than $10^{-12}$. In Figure 5.6 (right), a more detailed plot for times values $t \in\{1.1,1.2, \ldots, 2\}$ is shown.
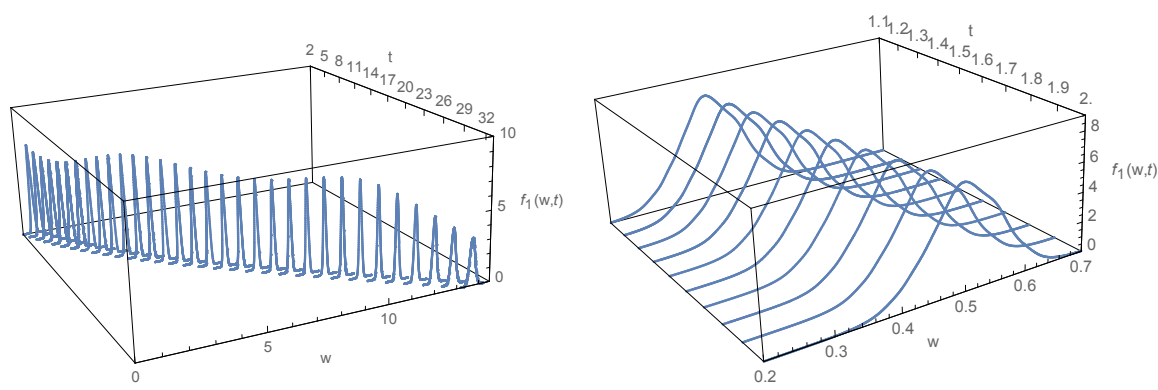

Figure 5.6: Left: 1-PDF of solution SP to random Bertalanffy model (5.10) given by (5.13) for all time of sample, $t \in\{2, \ldots, 33\}$. Model parameters are assumed to have multivariate Gaussian distribution defined by (5.20), (5.15) and (5.19). Right: Detailed representation of the 1-PDF for times $t \in\{1.1,1.2, \ldots, 2\}$.

The mean and the variance functions of fish weight over the time can be determined as follows

$$
\begin{aligned}
& \mu_{W}(t)=\mathbb{E}[W(t)]=\int_{-\infty}^{\infty} w f_{1}(w, t) \mathrm{d} w, \\
& \left(\sigma_{W}(t)\right)^{2}=\mathbb{V}[W(t)]=\int_{-\infty}^{\infty} w^{2} f_{1}(w, t) \mathrm{d} w-\left(\mu_{W}(t)\right)^{2},
\end{aligned}
$$


where $f_{1}(w, t)$ is defined by (5.13). In order to construct confidence intervals, first let us fix a time value $\hat{t} \geq 1$ and $\alpha \in(0,1)$, and secondly determine $\hat{w}_{1}=w_{1}(\hat{t})$ and $\hat{w}_{2}=w_{2}(\hat{t})$ such that

$$
\int_{0}^{\hat{w}_{1}} f_{1}(w, \hat{t}) \mathrm{d} w=\frac{\alpha}{2}=\int_{\hat{w}_{2}}^{1} f_{1}(w, \hat{t}) \mathrm{d} w .
$$

Then, $(1-\alpha) \times 100 \%$-confidence interval is specified by

$$
\left.1-\alpha=\mathbb{P}\left[\left\{\omega \in \Omega: W(\hat{t} ; \omega) \in\left[\hat{w}_{1}, \hat{w}_{2}\right)\right]\right\}\right]=\int_{\hat{w}_{1}}^{\hat{w}_{2}} f_{1}(w, \hat{t}) \mathrm{d} w .
$$

In Figure 5.7, mean function (solid line), 99\%-confidence intervals (dashed lines) and real data (points) are shown. From this graphical representation one observes that the proposed random Bertalanffy model captures satisfactorily data uncertainty.

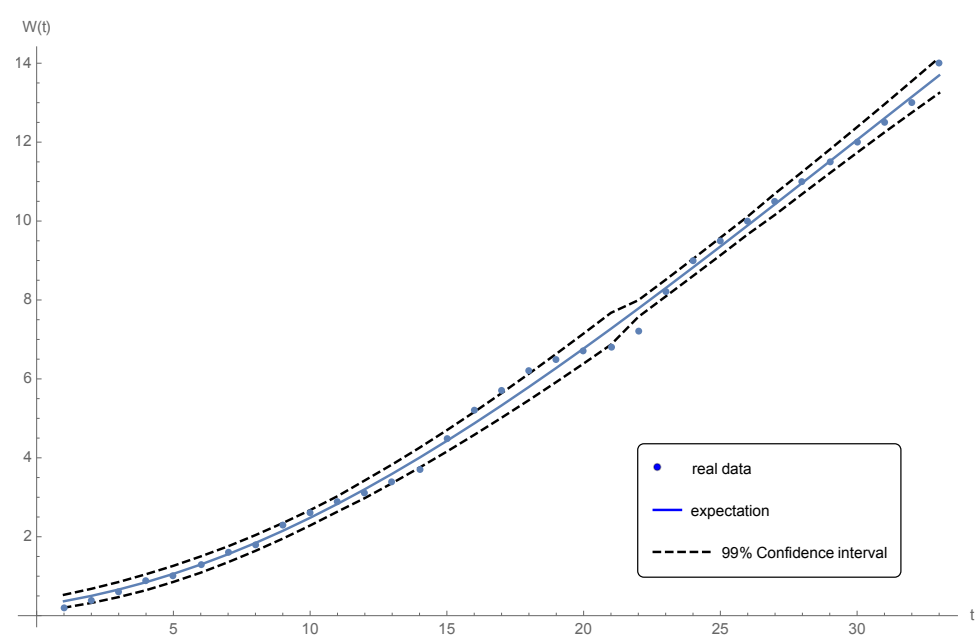

Figure 5.7: Expectation (solid line) and 99\%-confidence intervals (dotted lines). Points represent fish weigh. 


\subsection{Conclusions}

In this chapter Random Variable Transformation method has been successfully used to have a full probabilistic description of Bernoulli random differential equation. This description has been made through the computation of the first probability density function of solution stochastic process of that important equation. The study has been conducted in the general case that all input parameters of the equation as well as initial condition are absolutely continuous random variables. Two important features are that neither probabilistic independence among random variables nor specific probabilistic distributions have been assumed throughout the analysis. These facts provide a generality to the study. Therefore, any joint probability density function can be considered for model input parameters. Furthermore, it has been shown the usefulness of theoretical results obtained to model satisfactorily a real problem. Finally, notice that the proposed technique can be applied to compute the first probability density function of solution stochastic process for other random (ordinary or partial) differential equations. 



\section{Chapter 6}

\section{Random first-order linear systems of ordinary differential and difference equations}

In this chapter, the first probability density functions of solution stochastic processes to random autonomous first-order linear systems of ordinary differential and difference equations are determined. It is done under the general hypothesis that all coefficients and initial conditions are absolutely continuous random variables with an arbitrary joint probability density function. To differential equation theoretical results are illustrated for planar systems and a probabilistic interpretation of phase portrait is given. With respect to difference equation the finding of the first probability density function is applied to extend classical stability classification of zeroequilibrium point, based on the phase portrait, to random scenario. An example illustrates the potentiality of theoretical results established and their connection with their deterministic counterpart. 


\subsection{Introduction}

The chapter is organized as follows. In Section 6.2 a probabilistic solution of random autonomous first-order linear systems of ordinary differential equations will be provided. Subsection 6.2.2 construct an interpret the phase portrait of a planar autonomous first-order linear system of ordinary differential equation. The outline of Section 6.3 is the following. In Subsection 6.3.1 the 1-PDF of general random autonomous first-order linear homogeneous systems of difference equations is determined explicitly. In Subsection 6.3.2 a comprehensive probabilistic classification of equilibrium point for planar systems is given. In Subsection 6.3.3 an example illustrating the main theoretical results established in Section 6.3.2 is exhibited. Conclusions of both sections are drawn in Section 6.4 .

\subsection{Random autonomous first-order linear systems of ordinary differential equations}

In this section, RVT method, Theorem 2.1, will be used to determine, under very general hypotheses, the $1-\mathrm{PDF}$ of solution of random autonomous firstorder linear systems of ordinary differential equations of the form

$$
\begin{aligned}
\left.\begin{array}{rl}
\dot{\mathbf{X}}(t)= & \mathbf{A X}(t), t>t_{0},
\end{array}\right\} \quad \mathbf{X}_{0}=\left(X_{10}, \ldots, X_{n 0}\right), \\
\mathbf{X}\left(t_{0}\right)=\mathbf{X}_{0}, \\
\\
\mathbf{A}=\left(\begin{array}{ccc}
A_{11} & \cdots & A_{1 n} \\
\vdots & \ddots & \vdots \\
A_{n 1} & \cdots & A_{n n}
\end{array}\right),
\end{aligned}
$$

where the $n+n^{2}$ elements of $\mathbf{X}_{0}$ and $\mathbf{A}$ are assumed to be RVs with joint PDF

$$
f_{0}\left(x_{10}, \ldots, x_{n 0}, a_{11}, \ldots, a_{1 n}, \ldots, a_{n 1}, \ldots, a_{n n}\right),
$$

which, for convenience, in advance will be denoted by $f_{0}\left(\mathbf{x}_{0}, \mathbf{a}\right)$.

Notice that solution of random IVP (6.1) is given by $\exp \left(\mathbf{A}\left(t-t_{0}\right)\right) \mathbf{X}_{0}$. 


\subsubsection{Probabilistic solution}

In the following, it will be applied an extended version of Theorem 2.1 where component mappings $r_{i}$ (and hence $s_{i}$ ), $1 \leq i \leq m$, are vector transformations. With this aim, let us fix $t \geq t_{0}$ and denote by $\mathbf{e}_{i}=(0, \ldots, 0,1,0, \ldots, 0)^{\top}$ the $i$-th canonical vector of size $n, 1 \leq i \leq n$. Besides, let us define $h=$ $n+n^{2}=n(n+1)$ and the mapping $\mathbf{r}: \mathbb{R}^{h} \longrightarrow \mathbb{R}^{h}$ whose components $\left(\mathbf{r}_{1}\left(\mathbf{x}_{0}, \mathbf{a}\right), \ldots, \mathbf{r}_{n+1}\left(\mathbf{x}_{0}, \mathbf{a}\right)\right)$ are defined as follows

$$
\begin{aligned}
& \mathbf{y}_{1}=\mathbf{r}_{1}\left(\mathbf{x}_{0}, \mathbf{a}\right)=\exp \left(\mathbf{a}\left(t-t_{0}\right)\right) \mathbf{x}_{0}, \\
& \mathbf{y}_{2}=\mathbf{r}_{2}\left(\mathbf{x}_{0}, \mathbf{a}\right)=\mathbf{a e}_{1} \quad=\mathbf{a}_{1}, \\
& \begin{array}{lllll}
\vdots & \vdots & \vdots & \vdots & \vdots
\end{array} \\
& \mathbf{y}_{n}=\mathbf{r}_{n}\left(\mathbf{x}_{0}, \mathbf{a}\right)=\mathbf{a e}_{n-1} \quad=\mathbf{a}_{n-1}, \\
& \mathbf{y}_{n+1}=\mathbf{r}_{n+1}\left(\mathbf{x}_{0}, \mathbf{a}\right)=\mathbf{a} \mathbf{e}_{n} \quad=\mathbf{a}_{n} \text {. }
\end{aligned}
$$

Notice that $\mathbf{r}_{1}, \ldots, \mathbf{r}_{n+1}$ mappings are written in bold letters since they are vector transformations. In (6.2), $\mathbf{a}_{i}$ represents the $i$-th column of matrix $\mathbf{a}=$ $\mathbf{A}(\omega), 1 \leq i \leq n$, with $\omega \in \Omega$ arbitrary. Therefore, the inverse mapping, $\mathbf{s}$, of $\mathbf{r}$, is given by

$$
\begin{aligned}
& \mathbf{x}_{0}=\mathbf{s}_{1}\left(\mathbf{y}_{1}, \ldots, \mathbf{y}_{n+1}\right)=\exp \left(-\mathbf{a}\left(t-t_{0}\right)\right) \mathbf{y}_{1}, \\
& \mathbf{a}_{1}=\mathbf{s}_{2}\left(\mathbf{y}_{1}, \ldots, \mathbf{y}_{n+1}\right)=\mathbf{y}_{2}, \\
& \vdots \quad \vdots \quad \vdots \quad \vdots \quad \vdots \\
& \mathbf{a}_{n-1}=\mathbf{s}_{n}\left(\mathbf{y}_{1}, \ldots, \mathbf{y}_{n+1}\right)=\mathbf{y}_{n}, \\
& \mathbf{a}_{n}=\mathbf{s}_{n+1}\left(\mathbf{y}_{1}, \ldots, \mathbf{y}_{n+1}\right)=\mathbf{y}_{n+1},
\end{aligned}
$$

where, in the first equation, matrix a really depends on $\mathbf{y}_{1}, \ldots, \mathbf{y}_{n+1}$ since it can be equivalently represented by columns as $\mathbf{a}=\left(\mathbf{a}_{1}, \ldots, \mathbf{a}_{n}\right)=\left(\mathbf{y}_{2}, \ldots, \mathbf{y}_{n+1}\right)$. Now, the Jacobian of mapping $\mathbf{s}$ is computed 


$$
\begin{aligned}
& J_{h}=\operatorname{det}\left(\begin{array}{ccc}
\frac{\partial \mathbf{s}_{1}\left(\mathbf{y}_{1}, \ldots, \mathbf{y}_{n+1}\right)}{\partial \mathbf{y}_{1}} & \cdots & \frac{\partial \mathbf{s}_{n+1}\left(\mathbf{y}_{1}, \ldots, \mathbf{y}_{n+1}\right)}{\partial \mathbf{y}_{1}} \\
\vdots & \ddots & \vdots \\
\frac{\partial \mathbf{s}_{1}\left(\mathbf{y}_{1}, \ldots, \mathbf{y}_{n+1}\right)}{\partial \mathbf{y}_{n+1}} & \cdots & \frac{\partial \mathbf{s}_{n+1}\left(\mathbf{y}_{1}, \ldots, \mathbf{y}_{n+1}\right)}{\partial \mathbf{y}_{n+1}}
\end{array}\right)_{h \times h} \\
& =\operatorname{det}\left(\begin{array}{ccccc}
\exp \left(-\mathbf{a}\left(t-t_{0}\right)\right) & \mathbf{0}_{n} & \cdots & \cdots & \mathbf{0}_{n} \\
\mathbf{0}_{n} & \mathbf{I}_{n} & \ddots & & \vdots \\
\vdots & \ddots & \ddots & \ddots & \vdots \\
\vdots & & \ddots & \ddots & \mathbf{0}_{n} \\
\mathbf{0}_{n} & \cdots & \cdots & \mathbf{0}_{n} & \mathbf{I}_{n}
\end{array}\right)_{h \times h} \\
& =\exp \left(-\operatorname{tr}(\mathbf{a})\left(t-t_{0}\right)\right)>0,
\end{aligned}
$$

where $\operatorname{tr}(\mathbf{a})$ denotes the trace of matrix $\mathbf{a}$ and, as usually, $\mathbf{0}_{n}$ and $\mathbf{I}_{n}$ stand for null and identity matrices of size $n$, respectively. Therefore, applying Theorem 2.1 , the PDF of $n+1$ random vectors $\mathbf{Y}_{1}, \ldots, \mathbf{Y}_{n+1}$ defined by $(6.2)$ is given by

$$
\begin{aligned}
f_{\mathbf{Y}_{1}, \ldots, \mathbf{Y}_{n+1}}\left(\mathbf{y}_{1}, \ldots, \mathbf{y}_{n+1}\right)= & f_{0}\left(\exp \left(-\left(\mathbf{y}_{2}, \ldots, \mathbf{y}_{n+1}\right)\left(t-t_{0}\right)\right) \mathbf{y}_{1}, \mathbf{y}_{2}, \ldots, \mathbf{y}_{n+1}\right) \\
& \times \exp \left(-\operatorname{tr}\left(\left(\mathbf{y}_{2}, \ldots, \mathbf{y}_{n+1}\right)\right)\left(t-t_{0}\right)\right) .
\end{aligned}
$$

Since solution SP of random IVP (6.1) is given by the first component $\mathbf{y}_{1}$ of mapping $\mathbf{r}$ defined by $(6.2)$, its $1-\mathrm{PDF} f_{1}(\mathbf{x}, t)$ is obtained by marginalizing the expression (6.3) with respect to $\mathbf{Y}_{2}, \ldots, \mathbf{Y}_{n+1}$. Therefore, taking into account that $t \geq t_{0}$ is arbitrary, the following result has been established.

Theorem 6.1 Let us consider random IVP (6.1) and let $f_{0}\left(\mathbf{x}_{0}, \mathbf{a}\right)$ be the joint probability density function of $\mathbf{X}_{0}$ and $\mathbf{A}$, which are on a complete probability space $(\Omega, \mathcal{A}, \mathbb{P})$. Then, the first probability density function of solution stochastic process of IVP (6.1) is given by

$$
\begin{aligned}
f_{1}(\mathbf{x}, t)= & \int_{\mathbb{R}^{n^{2}}} f_{0}\left(\exp \left(-\mathbf{a}\left(t-t_{0}\right)\right) \mathbf{x}, \mathbf{a}_{1}, \ldots, \mathbf{a}_{n}\right) \\
& \times \exp \left(-\operatorname{tr}(\mathbf{a})\left(t-t_{0}\right)\right) \mathrm{d} a_{11} \cdots \mathrm{d} a_{n 1} \cdots \mathrm{d} a_{1 n} \cdots \mathrm{d} a_{n n},
\end{aligned}
$$


where $\mathbf{a}_{1}=\left(a_{11}, \ldots, a_{n 1}\right)^{\top}, \ldots, \mathbf{a}_{n}=\left(a_{1 n}, \ldots, a_{n n}\right)^{\top}$ denote the columns of matrix $\mathbf{a}=\mathbf{A}(\omega), \omega \in \Omega$.

Remark 6.1 Notice that this formula generalizes expression [16, Equation (47)] to matrix framework.

\subsubsection{An illustrative example}

As it has been pointed out in the Introduction (Chapter 1), the determination of the 1-PDF of a SP is advantageous. From it, many important statistical functions associated to the corresponding $\mathrm{SP}$ can be easily computed. For instance, mean, standard deviation functions as well as confidence intervals and the probability of certain sets of interest where SP lies can be obtained by the 1-PDF. In [16-18] one can found detailed examples where these quantities of interest are computed. However, in dealing with deterministic autonomous first-order linear systems of ordinary differential equations a distinctive feature is the study of their phase portrait. In the following, it will be shown how to take advantage of the theoretical result established in Theorem 6.1 to construct and interpret the phase portrait to random IVP (6.1). In order to show better results graphically, it will be done on case $n=2$, usually referred to as planar autonomous first-order linear systems of ordinary differential equations.

Example 6.1 Let us consider random IVP (6.1) with $t_{0}=0, n=2$ and

$$
\mathbf{X}(t)=\left(X_{1}(t), X_{2}(t)\right), \quad \mathbf{X}_{0}=\left(X_{10}, X_{20}\right), \quad \mathbf{A}=\left(\begin{array}{cc}
A_{11} & A_{12} \\
A_{21} & A_{22}
\end{array}\right)
$$

where $R V s X_{10}, X_{20}, A_{11}, A_{21}, A_{12}, A_{22}$ are assumed to be jointly Gaussian distributed with mean $(\mu)$ and variance-covariance matrix $(\Sigma)$ given by

$$
\mu=\left(\begin{array}{c}
1 \\
2 \\
-1 \\
-6 \\
1 \\
1 / 2
\end{array}\right), \quad \Sigma=\frac{1}{500}\left(\begin{array}{cccccc}
55 & 5 & 20 & 1 & 1 & 4 \\
5 & 20 & 5 & 10 & 2 & 4 \\
20 & 5 & 10 & 7 & 1 & 4 \\
1 & 10 & 7 & 30 & 2 & 4 \\
1 & 2 & 1 & 2 & 25 & 5 \\
4 & 4 & 4 & 4 & 5 & 10
\end{array}\right)
$$

In Figure 6.1, the probabilistic phase portrait of IVP (6.1) with input data (6.5)-(6.6) is shown. Spiral line represents the expectation of solution, i.e., 
$\mathbb{E}[\mathbf{X}(t)]=\left(\mathbb{E}\left[X_{1}(t)\right], \mathbb{E}\left[X_{2}(t)\right]\right)$. For each $t=\hat{t}>0$ arbitrary but fixed, these expectations have been computed by

$$
\mathbb{E}\left[X_{1}(\hat{t})\right]=\int_{\mathbb{R}^{2}} x_{1} f_{1}\left(x_{1}, x_{2}, \hat{t}\right) \mathrm{d} x_{1} \mathrm{~d} x_{2}, \quad \mathbb{E}\left[X_{2}(\hat{t})\right]=\int_{\mathbb{R}^{2}} x_{2} f_{1}\left(x_{1}, x_{2}, \hat{t}\right) \mathrm{d} x_{2} \mathrm{~d} x_{1},
$$

where $f_{1}\left(x_{1}, x_{2}, \hat{t}\right)$ is given by expression (6.4) and $f_{0}\left(\mathbf{x}_{0}, \mathbf{a}\right)$ is the multivariate $P D F$ of random vector $\mathbf{Z}$ with mean $\mu$ and variance-covariance matrix $\Sigma$ defined in (6.6), i.e.,

$$
\begin{aligned}
& f_{\mathbf{Z}}(\mathbf{z})=\frac{1}{\sqrt{(2 \pi)^{6} \operatorname{det}(\Sigma)}} \exp \left(-\frac{1}{2}(\mathbf{z}-\mu)^{\top} \Sigma^{-1}(\mathbf{z}-\mu)\right), \\
& \mathbf{z}=\left(x_{10}, x_{20}, a_{11}, a_{21}, a_{12}, a_{22}\right) .
\end{aligned}
$$

Notice that trajectory shown in the phase portrait starts at point $\left(\mu_{1}, \mu_{2}\right)=$ $\left(\mathbb{E}\left[X_{10}\right], \mathbb{E}\left[X_{20}\right]\right)=(1,2)$. In Figure $6.1,50 \%$ and $90 \%$ confidence regions at several fixed time instants, $\hat{t} \in\{0,0.5,1\}$, have been also plotted. The greater the region, the higher the confidence level. For $t=\hat{t}>0$ and $\alpha \in(0,1)$ fixed, each one of these $(1-\alpha) \times 100 \%$ confidence regions, $\mathcal{D}_{\mathbf{X}(\hat{t})}(1-\alpha) \subset \mathbb{R}^{2}$, has been determined as the $\left(x_{1}, x_{2}\right)$-curve such that

$$
\begin{aligned}
& 1-\alpha=\iint_{\mathcal{D}_{\mathbf{X}(\hat{t})}(1-\alpha)} f_{1}\left(x_{1}, x_{2} ; \hat{t}\right) \mathrm{d} x_{1} \mathrm{~d} x_{2}, \\
& \mathcal{D}_{\mathbf{X}(\hat{t})}(1-\alpha)=\left\{\left(x_{1}, x_{2}\right): f_{1}\left(x_{1}, x_{2} ; \hat{t}\right)=k\right\} .
\end{aligned}
$$

Observe that $50 \%$ (blue curve) and $90 \%$ confidence (red curve) regions plotted in Figure 6.1 correspond to $\alpha=0.5$ and $\alpha=0.1$, respectively. Null random vector $(0,0)$ is a stable spiral point. This fact can be connected with the wellknown classical theory of dynamic systems. To this end, let us consider the deterministic IVP

$$
\begin{aligned}
& \left.\begin{array}{l}
\dot{\mathbf{h}}(t)=\mathcal{M} \mathbf{h}(t), t>0, \\
\mathbf{h}(0)=\mathbf{h}_{0},
\end{array}\right\} \quad \mathbf{h}_{0}=\left(\begin{array}{l}
\mathbb{E}\left[X_{10}\right] \\
\mathbb{E}\left[X_{20}\right]
\end{array}\right)=\left(\begin{array}{l}
1 \\
2
\end{array}\right), \\
& \mathcal{M}=\left(\begin{array}{ll}
\mathbb{E}\left[A_{11}\right] & \mathbb{E}\left[A_{12}\right] \\
\mathbb{E}\left[A_{21}\right] & \mathbb{E}\left[A_{22}\right]
\end{array}\right)=\left(\begin{array}{cc}
-1 & 1 \\
-6 & 1 / 2
\end{array}\right),
\end{aligned}
$$



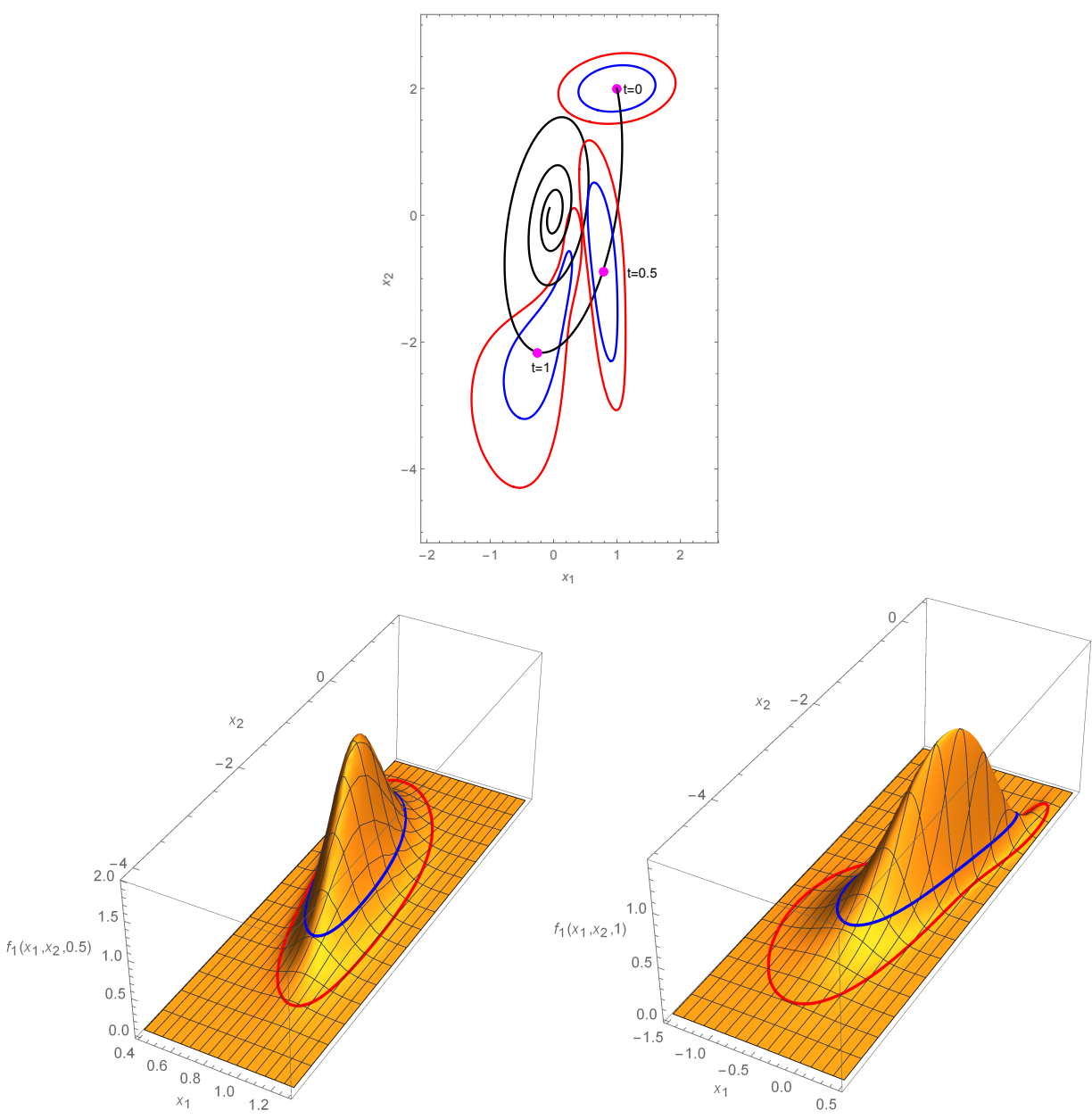

Figure 6.1: Top: Phase portrait. Continuous (black) spiral line represents the expectation of solution. $50 \%$ (blue curve) and $90 \%$ (red curve) confidence regions are plotted at time instants $\hat{t} \in\{0,0.5,1\}$. Bottom left: PDF of solution at time instant $\hat{t}=0.5$. Bottom right: PDF of solution at time instant $\hat{t}=1$. On the PDFs plots $50 \%$ and $90 \%$ confidence regions have been highlighted. All these graphical representations correspond to Example 6.1. 
whose coefficients and initial conditions are the expectation of the corresponding RVs defining random IVP (6.1) and (6.5)-(6.6). Thus, these mean values are defined by the vector $\mu$ defined in (6.6). Since eigenvalues of the deterministic matrix $\mathcal{M}$ are $-1 / 4 \pm i \sqrt{87} / 4$, hence having negative real part, equilibrium point $(0,0)$ is expected to be a stable spiral node in random scenario. However, it really occurs with certain probability, say $p$, that needs to be determined. As a nice application of determining the 1-PDF of solution SP to random IVP (6.1) and (6.5)-(6.6), in the following the value of probability $p$ is computed.

Since $A_{i j}, 1 \leq i, j \leq 2$ are continuous $R V s$, $\operatorname{det}(\mathbf{A})(\omega)=A_{11}(\omega) A_{22}(\omega)-$ $A_{12}(\omega) A_{21}(\omega) \neq 0$ for all $\omega \in \Omega$ w.p. 1. Hence, null vector $(0,0)$ is the only equilibrium point to IVP (6.5) w.p. 1. Notice that random characteristic roots to this random IVP are given by

$$
\lambda^{2}-\operatorname{tr}(\mathbf{A}) \lambda+\operatorname{det}(\mathbf{A})=0, \quad \lambda=\frac{\operatorname{tr}(\mathbf{A}) \pm \sqrt{(\operatorname{tr}(\mathbf{A}))^{2}-4 \operatorname{det}(\mathbf{A})}}{2} .
$$

Thus, the probability $p$ that random point $(0,0)$ be stable spiral point is given by

$$
\begin{aligned}
p & =\mathbb{P}\left[\left\{\omega \in \Omega:\left(V_{1}(\omega)\right)^{2}-4 V_{2}(\omega)<0, V_{1}(\omega)<0\right\}\right] \\
& =\int_{-\infty}^{0} \int_{\left(v_{1}\right)^{2} / 4}^{+\infty} f_{V_{1}, V_{2}}\left(v_{1}, v_{2}\right) \mathrm{d} v_{2} \mathrm{~d} v_{1},\left\{\begin{array}{l}
V_{1}(\omega)=\operatorname{tr}(\mathbf{A}(\omega)), \\
V_{2}(\omega)=\operatorname{det}(\mathbf{A}(\omega)) .
\end{array}\right.
\end{aligned}
$$

In order to compute this probability, the joint PDF of RVs $V_{1}$ and $V_{2}$ defined in (6.8) needs to be previously computed. To that end, let us define the following transformation $\mathbf{r}: \mathbb{R}^{4} \longrightarrow \mathbb{R}^{4}$,

$$
\begin{aligned}
& V_{1}=r_{1}\left(A_{11}, A_{21}, A_{12}, A_{22}\right)=A_{11}+A_{22}, \\
& V_{2}=r_{2}\left(A_{11}, A_{21}, A_{12}, A_{22}\right)=A_{11} A_{22}-A_{12} A_{21}, \\
& V_{3}=r_{3}\left(A_{11}, A_{21}, A_{12}, A_{22}\right)=A_{12}, \\
& V_{4}=r_{4}\left(A_{11}, A_{21}, A_{12}, A_{22}\right)=A_{22},
\end{aligned}
$$

being the inverse transformation $\mathbf{s}: \mathbb{R}^{4} \longrightarrow \mathbb{R}^{4}$ of mapping $\mathbf{r}$ 


$$
\begin{aligned}
& A_{11}=s_{1}\left(V_{1}, V_{2}, V_{3}, V_{4}\right)=V_{1}-V_{4}, \\
& A_{21}=s_{2}\left(V_{1}, V_{2}, V_{3}, V_{4}\right)=-\frac{V_{2}-\left(V_{1}-V_{4}\right) V_{4}}{V_{3}}, \\
& A_{12}=s_{3}\left(V_{1}, V_{2}, V_{3}, V_{4}\right)=V_{3}, \\
& A_{22}=s_{4}\left(V_{1}, V_{2}, V_{3}, V_{4}\right)=V_{4} .
\end{aligned}
$$

The Jacobian of $\mathbf{s}$ is given by

$$
J_{4}=\operatorname{det}\left(\begin{array}{cccc}
1 & \frac{1}{v_{3}} & 0 & 0 \\
0 & -\frac{1}{v_{3}} & 0 & 0 \\
0 & \frac{v_{2}-\left(v_{1}-v_{4}\right) v_{4}}{\left(v_{3}\right)^{2}} & 1 & 0 \\
-1 & -\frac{2 v_{4}}{v_{3}} & 0 & 1
\end{array}\right)=-\frac{1}{v_{3}} \neq 0
$$

Therefore, the joint PDF of random vector $\left(V_{1}, V_{2}\right)=(\operatorname{tr}(\mathbf{A})$, $\operatorname{det}(\mathbf{A}))$ is given by

$$
\begin{aligned}
& f_{V_{1}, V_{2}}\left(v_{1}, v_{2}\right)= \\
& \quad \int_{\mathbb{R}^{2}} f_{A_{11}, A_{21}, A_{12}, A_{22}}\left(v_{1}-a_{22},-\frac{v_{2}-\left(v_{1}-a_{22}\right) a_{22}}{a_{12}}, a_{12}, a_{22}\right) \frac{1}{a_{12}} \mathrm{~d} a_{12} \mathrm{~d} a_{22},
\end{aligned}
$$

where

$$
f_{A_{11}, A_{21}, A_{12}, A_{22}}\left(a_{11}, a_{12}, a_{21}, a_{22}\right)=\int_{\mathbb{R}^{2}} f_{\mathbf{Z}}\left(x_{10}, x_{20}, a_{11}, a_{21}, a_{12}, a_{22}\right) \mathrm{d} x_{10} \mathrm{~d} x_{20},
$$

being $f_{\mathbf{Z}}\left(x_{10}, x_{20}, a_{11}, a_{21}, a_{12}, a_{22}\right)=f_{\mathbf{z}}(\mathbf{z})$ defined by (6.7). After carrying out computations, the value of $p$ defined in (6.8) is 0.983. It accounts for the probability that equilibrium point $(0,0)$ be a stable spiral point.

\subsection{Random autonomous first-order linear systems of difference equations}

The aim of this section is twofold. Firstly, to determine the 1-PDF of solution SP of random autonomous first-order linear systems of difference equations of arbitrary size, say $m$. Secondly, to extend the main deterministic results on stability for planar systems $(m=2)$ to random scenario. This section is 
heavily inspired in the previous contribution in this chapter, but having two main differences. Firstly, it is considered random difference equations instead of random differential equations. Secondly, it will be provided a comprehensive probabilistic stability classification of zero-equilibrium point of random planar homogeneous systems rather than just to illustrate the classification with one example. The interest of this analysis is expected to reach a large audience for two main reasons. On the one hand, the study provides a generalization to random framework of deterministic autonomous difference equations, which have mathematical interest by themselves. Indeed, for instance, these class of equations become after discretizating autonomous differential equations. On the other hand, it is well-known that autonomous difference equations are adequate choice when modelling many real phenomena. Therefore, it is expected that consideration of randomness into autonomous difference equations will provide more realistic models in applications.

\subsubsection{Probabilistic solution}

The goal of this subsection is to compute an explicit formula for the 1-PDF of random autonomous first-order homogeneous linear systems of difference equations

$$
\mathbf{X}_{n+1}=\mathbf{A X}_{n}, n \geq 0, \quad \mathbf{A}=\left(A_{i j}\right), 1 \leq i, j \leq m
$$

where $A_{i j}, 1 \leq i, j \leq m$, and $X_{i 0}, 1 \leq i \leq m$, that define the starting seed $\mathbf{X}_{0}=\left(X_{10}, \ldots, X_{m 0}\right)$, are $h=m+m^{2}$ absolutely continuous RVs defined in a complete probabilistic space $(\Omega, \mathcal{A}, \mathbb{P})$. It is assumed that these RVs have the following joint PDF

$$
f_{0}\left(\mathbf{x}_{0}, \mathbf{a}\right)=f_{0}\left(x_{10}, \ldots, x_{m 0}, a_{11}, \ldots, a_{m 1}, \ldots, a_{1 m}, \ldots, a_{m m}\right) .
$$

Hereinafter, it will be assumed that random matrix $\mathbf{A}$ is invertible in the probabilistic sense, i.e., $\mathbb{P}[\{\omega \in \Omega: \operatorname{det}(\mathbf{A}(\omega)) \neq 0\}]=1$. Notice that this hypothesis is not restrictive since $A_{i j}$ are assumed to be absolutely continuous RVs.

Let us observe that solution of (6.10) is given by $\mathbf{X}_{n}=\mathbf{A}^{n} \mathbf{X}_{0}$. Now, it will be applied RVT technique as stated in Theorem 2.1. With this aim, let us fix $n>0$ and denote by $\mathbf{e}_{i}=(0, \ldots, 0,1,0, \ldots, 0)^{\top}$ the $i$-th canonical vector of size $m, 1 \leq i \leq m$. Additionally, let us also define the transformation 
$\mathbf{r}: \mathbb{R}^{h} \rightarrow \mathbb{R}^{h}$, and the inverse mapping of $\mathbf{r}, \mathbf{s}=\mathbf{r}^{-1}$, whose components are given by

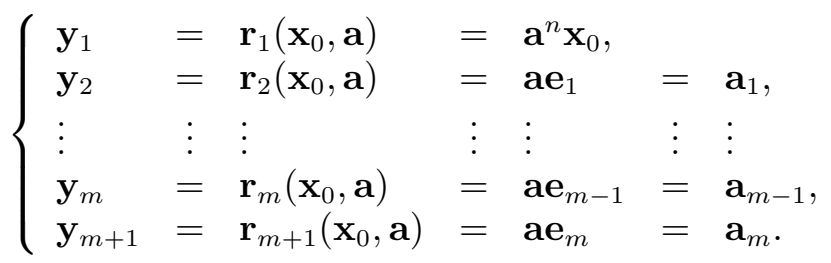

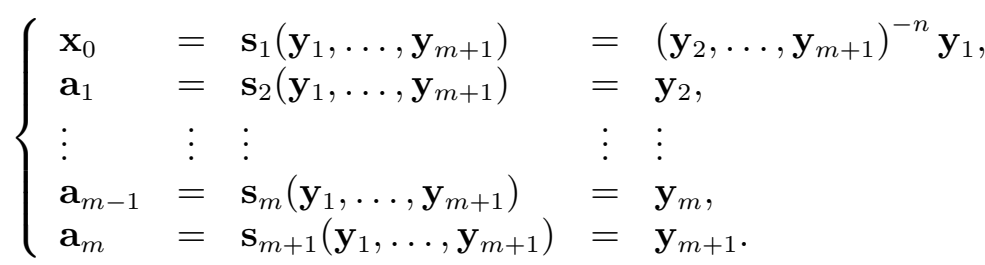

Notice that in (6.11) $\mathbf{a}_{i}$ represents the $i$-th column of matrix $\mathbf{a}=\mathbf{A}(\omega), \omega \in \Omega$. Now, the Jacobian, which is defined by the following determinant, is computed

$$
\begin{aligned}
J_{h}=\operatorname{det}\left(\begin{array}{ccccc}
\left(\mathbf{y}_{2}, \ldots, \mathbf{y}_{m+1}\right)^{-n} & \mathbf{0}_{m} & \cdots & \cdots & \mathbf{0}_{m} \\
\mathbf{0}_{m} & \mathbf{I}_{m} & \ddots & & \vdots \\
\vdots & \ddots & \ddots & \ddots & \vdots \\
\vdots & & \ddots & \ddots & \mathbf{0}_{m} \\
\mathbf{0}_{m} & \cdots & \cdots & \mathbf{0}_{m} & \mathbf{I}_{m}
\end{array}\right)_{h \times h} \\
=\operatorname{det}\left(\left(\mathbf{y}_{2}, \ldots, \mathbf{y}_{m+1}\right)^{-n}\right)=\left(\operatorname{det}\left(\left(\mathbf{y}_{2}, \ldots, \mathbf{y}_{m+1}\right)\right)\right)^{-n}=(\operatorname{det}(\mathbf{a}))^{-n} \neq 0,
\end{aligned}
$$

where $\mathbf{0}_{m}$ and $\mathbf{I}_{m}$ are the null and the identity matrix of size $m$, respectively. Notice that $\operatorname{det}(\mathbf{a}) \neq 0$ a.s. Therefore, applying Therorem 2.1, the joint PDF of random vector $\left(\mathbf{Y}_{1}, \ldots, \mathbf{Y}_{m+1}\right)$ is obtained

$$
\begin{aligned}
f_{\mathbf{Y}_{1}, \ldots, \mathbf{Y}_{m+1}}\left(\mathbf{y}_{1}, \ldots, \mathbf{y}_{m+1}\right)= & f_{0}\left(\left(\mathbf{y}_{2}, \ldots, \mathbf{y}_{m+1}\right)^{-n} \mathbf{y}_{1}, \mathbf{y}_{2}, \ldots, \mathbf{y}_{m+1}\right) \\
& \times\left|\operatorname{det}\left(\left(\mathbf{y}_{2}, \ldots, \mathbf{y}_{m+1}\right)\right)\right|^{-n} .
\end{aligned}
$$

As solution of IVP (6.10) is given by the first component of random vector $\left(\mathbf{Y}_{1}, \ldots, \mathbf{Y}_{m+1}\right)$, in order to compute the PDF of solution, $\mathbf{X}_{n}$, first expression 
(6.12) must be marginalized with respect to $\mathbf{Y}_{2}, \ldots, \mathbf{Y}_{m+1}$, and secondly to express the result in terms of data. This yields

$$
f_{1}(\mathbf{x}, n)=\int_{\mathbb{R}^{m^{2}}} f_{0}\left(\mathbf{a}^{-n} \mathbf{x}, \mathbf{a}_{1}, \ldots, \mathbf{a}_{m}\right)|\operatorname{det}(\mathbf{a})|^{-n} \mathrm{~d} a_{11} \cdots \mathrm{d} a_{m 1} \cdots \mathrm{d} a_{1 m} \cdots \mathrm{d} a_{m m} .
$$

\subsubsection{Random planar autonomous first-order linear systems: A probabilistic classification of equilibrium point}

This subsection is devoted to classify, from a probabilistic standpoint, equilibrium point of random system of difference equations (6.10), when $m=2$. Observe that the analysis is restricted to the homogeneous case where the only equilibrium point is random null vector, $\mathbf{X}_{\mathrm{e}}=\mathbf{0}$. Nevertheless, nonhomogeneous case, $\mathbf{X}_{n+1}=\mathbf{A} \mathbf{X}_{n}+\mathbf{B}$, can be reduced to homogeneous one, just taking into account Equation (6.10) can be centered about $\mathbf{X}_{\mathrm{e}}=\left(\mathbf{I}_{m}-\mathbf{A}\right)^{-1} \mathbf{B}$. Indeed, this can be done because the probability that RV $\lambda=1$ be an eigenvalue of $\mathbf{A}$ is zero. The case where $m=2$ in random matrix difference equation (6.10) corresponds to random planar systems. In the deterministic context, it is well-known that important results related to stability of zero-equilibrium point have been established for planar systems. This subsection is addressed to extend classical stability classification of zero-equilibrium $\mathbf{X}_{\mathrm{e}}=(0,0)$ to random scenario. As it shall see later, this approach leads to a nice generalization, in a probabilistic sense, that retains the well-known deterministic results when probabilistic events associated to that classification happens w.p. 1. Naturally, as it also occurs in the deterministic case, classification depends on characteristic roots, $\lambda_{1}$ and $\lambda_{2}$, associated to random matrix equation (6.10) with $m=2$. These roots can be expressed in terms of trace and the determinant of random matrix $\mathbf{A}$, as in previous section

$$
\lambda^{2}-\operatorname{tr}(\mathbf{A}) \lambda+\operatorname{det}(\mathbf{A})=0, \quad \lambda_{i}=\frac{\operatorname{tr}(\mathbf{A}) \pm \sqrt{(\operatorname{tr}(\mathbf{A}))^{2}-4 \operatorname{det}(\mathbf{A})}}{2}, \quad i=1,2
$$

Denoting by $V_{1}(\omega)=\operatorname{tr}(\mathbf{A}(\omega))$ and $V_{2}(\omega)=\operatorname{det}(\mathbf{A}(\omega))$, for each $\omega \in \Omega$, the classification can be represented by means of Figure 6.2. Then taking advantage of the 1-PDF of solution SP of (6.10), given by (6.13), one can compute the probability that zero-equilibrium or critical point belongs to one of the following states: stable (node or sink/spiral), unstable (node or source/spiral) or saddle point. The case that zero-equilibrium point be a center, an improper 
stable node or an improper unstable node have been neglected because they are associated to events $A_{1}, A_{2}$ and $A_{3}$ defined by

$$
\begin{aligned}
& A_{1}=\{\omega \in \Omega: \operatorname{det}(\mathbf{A}(\omega))=1,|\operatorname{tr}(\mathbf{A}(\omega))| \leq 2\}, \\
& A_{2}=\left\{\omega \in \Omega:(\operatorname{tr}(\mathbf{A}(\omega)))^{2}=4 \operatorname{det}(\mathbf{A}(\omega)),|\operatorname{tr}(\mathbf{A}(\omega))|<2\right\}, \\
& A_{3}=\left\{\omega \in \Omega:(\operatorname{tr}(\mathbf{A}(\omega)))^{2}=4 \operatorname{det}(\mathbf{A}(\omega)),|\operatorname{tr}(\mathbf{A}(\omega))|>2\right\},
\end{aligned}
$$

respectively, which can happens with probability zero since $A_{i j}, 1 \leq i, j \leq 2$, are assumed to be absolutely continuous RVs. Below, these probabilities are completely specified. It is important to point out that, in contrasts to what happens in the deterministic scenario, where equilibrium point can only belong to one state of the previous list, in random context the situation is different since zero-equilibrium point can have different states but each one with different probabilities.

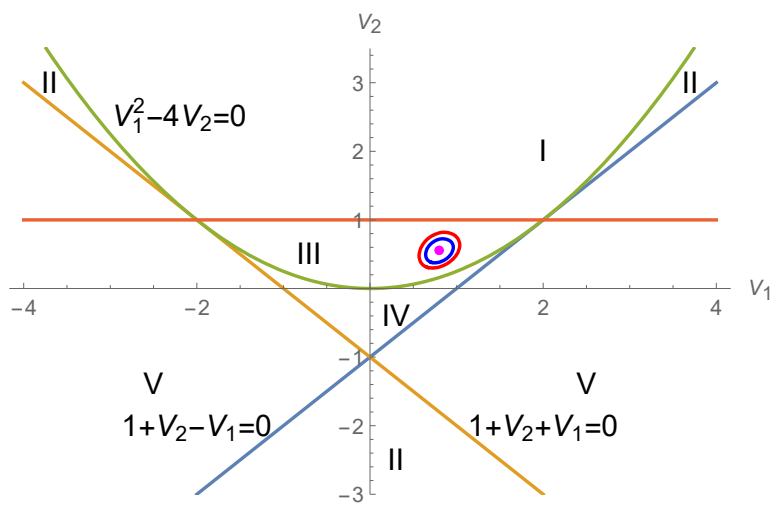

Figure 6.2: Graphical representation of the stability classification of zero-equilibrium point to random matrix difference equation (6.10) with $m=2$ (random planar system). Each relevant region has a label corresponding to: I (unstable spiral); II (unstable node or source); III (stable spiral); IV (stable node or sink) and V (saddle point). Magenta point has been obtained from matrix $\overline{\mathbf{a}}$ of averaged system (6.16) associated to random system (6.10) with $m=2$ and random inputs given by (6.14)-(6.15). Blue-red confidence regions have been determined from (6.9).

- Stable node:

$$
\mathrm{P}_{\mathrm{sn}}=\int_{-2}^{0} \int_{-1-v_{1}}^{\frac{v_{1}^{2}}{4}} f_{V_{1}, V_{2}}\left(v_{1}, v_{2}\right) \mathrm{d} v_{2} \mathrm{~d} v_{1}+\int_{0}^{2} \int_{-1+v_{1}}^{\frac{v_{1}^{2}}{4}} f_{V_{1}, V_{2}}\left(v_{1}, v_{2}\right) \mathrm{d} v_{2} \mathrm{~d} v_{1} .
$$


- Stable spiral:

$$
\mathrm{P}_{\mathrm{ss}}=\int_{-2}^{2} \int_{\frac{v_{1}^{2}}{4}}^{1} f_{V_{1}, V_{2}}\left(v_{1}, v_{2}\right) \mathrm{d} v_{2} \mathrm{~d} v_{1} .
$$

- Unstable node:

$$
\begin{aligned}
\mathrm{P}_{\text {un }}= & \int_{-\infty}^{-2} \int_{-1-v_{1}}^{\frac{v_{1}^{2}}{4}} f_{V_{1}, V_{2}}\left(v_{1}, v_{2}\right) \mathrm{d} v_{2} \mathrm{~d} v_{1}+\int_{2}^{\infty} \int_{-1+v_{1}}^{\frac{v_{1}^{2}}{4}} f_{V_{1}, V_{2}}\left(v_{1}, v_{2}\right) \mathrm{d} v_{2} \mathrm{~d} v_{1} \\
& +\int_{-\infty}^{-1} \int_{1+v_{2}}^{-1-v_{2}} f_{V_{1}, V_{2}}\left(v_{1}, v_{2}\right) \mathrm{d} v_{1} \mathrm{~d} v_{2} .
\end{aligned}
$$

- Unstable spiral:

$$
\mathrm{P}_{\text {us }}=\int_{1}^{\infty} \int_{-2 \sqrt{v_{2}}}^{2 \sqrt{v_{2}}} f_{V_{1}, V_{2}}\left(v_{1}, v_{2}\right) \mathrm{d} v_{1} \mathrm{~d} v_{2}
$$

- Saddle point:

$$
\mathrm{P}_{\mathrm{s}}=\int_{-\infty}^{0} \int_{-1+v_{1}}^{-1-v_{1}} f_{V_{1}, V_{2}}\left(v_{1}, v_{2}\right) \mathrm{d} v_{2} \mathrm{~d} v_{1}+\int_{0}^{\infty} \int_{-1-v_{1}}^{-1+v_{1}} f_{V_{1}, V_{2}}\left(v_{1}, v_{2}\right) \mathrm{d} v_{2} \mathrm{~d} v_{1} .
$$

All these probabilities depend on the PDF, $f_{V_{1}, V_{2}}\left(v_{1}, v_{2}\right)$, of random vector $\left(V_{1}, V_{2}\right)=(\operatorname{tr}(\mathbf{A}), \operatorname{det}(\mathbf{A}))$. Applying Theorem 2.1, that is to say, RVT method, one can determine $f_{V_{1}, V_{2}}\left(v_{1}, v_{2}\right)$. To this end, let us define the following mapping, $\mathbf{r}: \mathbb{R}^{4} \longrightarrow \mathbb{R}^{4}$, whose inverse mapping $\mathbf{s}: \mathbb{R}^{4} \longrightarrow \mathbb{R}^{4}$ is given by

$$
\begin{aligned}
& \left\{\begin{array}{l}
v_{1}=r_{1}\left(a_{11}, a_{21}, a_{12}, a_{22}\right)=a_{11}+a_{22}, \\
v_{2}=r_{2}\left(a_{11}, a_{21}, a_{12}, a_{22}\right)=a_{11} a_{22}-a_{12} a_{21}, \\
v_{3}=r_{3}\left(a_{11}, a_{21}, a_{12}, a_{22}\right)=a_{12}, \\
v_{4}=r_{4}\left(a_{11}, a_{21}, a_{12}, a_{22}\right)=a_{22},
\end{array}\right. \\
& \left\{\begin{array}{l}
a_{11}=s_{1}\left(v_{1}, v_{2}, v_{3}, v_{4}\right)=v_{1}-v_{4}, \\
a_{21}=s_{2}\left(v_{1}, v_{2}, v_{3}, v_{4}\right)=-\frac{v_{2}-\left(v_{1}-v_{4}\right) v_{4}}{v_{3}}, \\
a_{12}=s_{3}\left(v_{1}, v_{2}, v_{3}, v_{4}\right)=v_{3}, \\
a_{22}=s_{4}\left(v_{1}, v_{2}, v_{3}, v_{4}\right)=v_{4} .
\end{array}\right.
\end{aligned}
$$


It is easy to check that the Jacobian of $\mathbf{s}$ is $J_{4}=-1 / v_{3} \neq 0$. Therefore, the joint PDF of random vector $\left(V_{1}, V_{2}\right)=(\operatorname{tr}(\mathbf{A}), \operatorname{det}(\mathbf{A}))$ is given by

$$
\begin{aligned}
& f_{V_{1}, V_{2}}\left(v_{1}, v_{2}\right) \\
& =\int_{\mathbb{R}^{2}} f_{A_{11}, A_{21}, A_{12}, A_{22}}\left(v_{1}-a_{22},-\frac{v_{2}-\left(v_{1}-a_{22}\right) a_{22}}{a_{12}}, a_{12}, a_{22}\right) \frac{1}{\left|a_{12}\right|} \mathrm{d} a_{12} \mathrm{~d} a_{22},
\end{aligned}
$$

being

$$
f_{A_{11}, A_{21}, A_{12}, A_{22}}\left(a_{11}, a_{21}, a_{12}, a_{22}\right)=\int_{\mathbb{R}^{2}} f_{0}\left(x_{10}, x_{20}, a_{11}, a_{21}, a_{12}, a_{22}\right) \mathrm{d} x_{10} \mathrm{~d} x_{20} .
$$

\subsubsection{An illustrative example}

The aim of this subsection is to illustrate the theoretical results previously established by means an example. This includes the interpretation of the phase portrait in the context of random planar systems of the form (6.10) with $m=2$ and the previous probabilistic stability classification of zero-equilibrium point. It will be considered that

$$
\mathbf{Z}=\left(X_{10}, X_{20}, A_{11}, A_{21}, A_{12}, A_{22}\right), \quad \mathbf{Z} \sim \mathrm{N}(\mu, \Sigma)
$$

where the mean, $\mu$, and the variance-covariance matrix, $\Sigma$, are,

$$
\mu=\left(\begin{array}{c}
2 \\
2 \\
-0.125 \\
-0.962 \\
0.692 \\
0.925
\end{array}\right), \Sigma=\frac{1}{4000}\left(\begin{array}{cccccc}
55 & 5 & 20 & 1 & 1 & 4 \\
5 & 20 & 5 & 10 & 2 & 4 \\
20 & 5 & 10 & 7 & 1 & 4 \\
1 & 10 & 7 & 30 & 2 & 4 \\
1 & 2 & 1 & 2 & 25 & 5 \\
4 & 4 & 4 & 4 & 5 & 10
\end{array}\right)
$$

In Figure 6.3 it is shown the phase portrait for different fixed time instants, $n \in\{0,1,2,3\}$, together with the PDF of solution SP in two of these time instants $(n \in\{2,3\})$. In the planar phase portrait, mean $\mathbb{E}\left[X_{i n}\right], i=1,2$, and confidence regions $\mathcal{D}_{\mathbf{X}_{n}}(1-\alpha) \subset \mathbb{R}^{2}$ at different fixed levels of confidence, $\alpha \in\{0.50,0.90\} \in(0,1)$, for $n=\{2,3\}$, have been plotted. These statistical quantities have been computed by means of the following expressions 


$$
\begin{gathered}
\mathbb{E}\left[X_{1 n}\right]=\int_{\mathbb{R}^{2}} x_{1} f_{1}\left(x_{1}, x_{2}, n\right) \mathrm{d} x_{1} \mathrm{~d} x_{2}, \\
\mathbb{E}\left[X_{2 n}\right]=\int_{\mathbb{R}^{2}} x_{2} f_{1}\left(x_{1}, x_{2}, n\right) \mathrm{d} x_{2} \mathrm{~d} x_{1}, \\
1-\alpha=\iint_{\mathcal{D}_{\mathbf{x}_{n}}(1-\alpha)} f_{1}\left(x_{1}, x_{2}, n\right) \mathrm{d} x_{1} \mathrm{~d} x_{2}, \\
\mathcal{D}_{\mathbf{X}_{n}}(1-\alpha)=\left\{\left(x_{1}, x_{2}\right): f_{1}\left(x_{1}, x_{2}, n\right)=k\right\},
\end{gathered}
$$

where, in agreement with (6.13),

$$
\begin{aligned}
f_{1}\left(x_{1}, x_{2} ; n\right)= & \int_{\mathbb{R}^{4}} f_{0}\left(\left(\begin{array}{ll}
a_{11} & a_{12} \\
a_{21} & a_{22}
\end{array}\right)^{-n}\left(\begin{array}{l}
x_{1} \\
x_{2}
\end{array}\right), a_{11}, a_{21}, a_{12}, a_{22}\right) \\
& \times\left|a_{11} a_{22}-a_{12} a_{21}\right|^{-n} \mathrm{~d} a_{11} \mathrm{~d} a_{12} \mathrm{~d}_{21} \mathrm{~d}_{22} .
\end{aligned}
$$

As it has been indicated previously, zero-equilibrium point can behaves different classification in random context with different probabilities. Based upon analysis, these probabilities can be quantified. In this example, the probability that the null random point $(0,0)$ be either a saddle point, a stable node/sink or an unstable node is 0 . While the probability of being a stable spiral is 0.999995 and the probability of being a unstable spiral is 0.000005 . The fact that equilibrium point is more likely a stable spiral than an unstable spiral is heavily connected with the deterministic theory. Indeed, let us consider the averaged problem associated to (6.10)

$$
\overline{\mathbf{x}}_{n+1}=\overline{\mathbf{a}} \overline{\mathbf{x}}_{n}, \quad n \geq 0, \quad \overline{\mathbf{a}}=\left(\begin{array}{ll}
-0.125 & 0.692 \\
-0.962 & 0.925
\end{array}\right)
$$

and starting value $\overline{\mathbf{x}}_{0}=(2,2)$. Observe that $\overline{\mathbf{a}}$ and $\overline{\mathbf{x}}_{0}$ correspond to the expectation or average defined in (6.15). For the deterministic problem (6.16) one gets

$$
\begin{gathered}
\operatorname{tr}(\overline{\mathbf{a}})^{2}-4 \operatorname{det}(\overline{\mathbf{a}})=0.8^{2}-40.55=-1.56<0, \\
-1+\operatorname{det}(\overline{\mathbf{a}})=-1+0.55=-0.45<0 .
\end{gathered}
$$


Therefore, equilibrium point $(0,0)$ is a stable spiral. In Figure 6.2 it have been plotted the magenta point $(\operatorname{det}(\overline{\mathbf{a}}), \operatorname{tr}(\overline{\mathbf{a}}))=(0.55,0.8)$ and confidence regions (blue-red rings) at 50\% and 90\% confidence levels, respectively. One observes that this plot is in agreement with the probabilistic results previously shown.
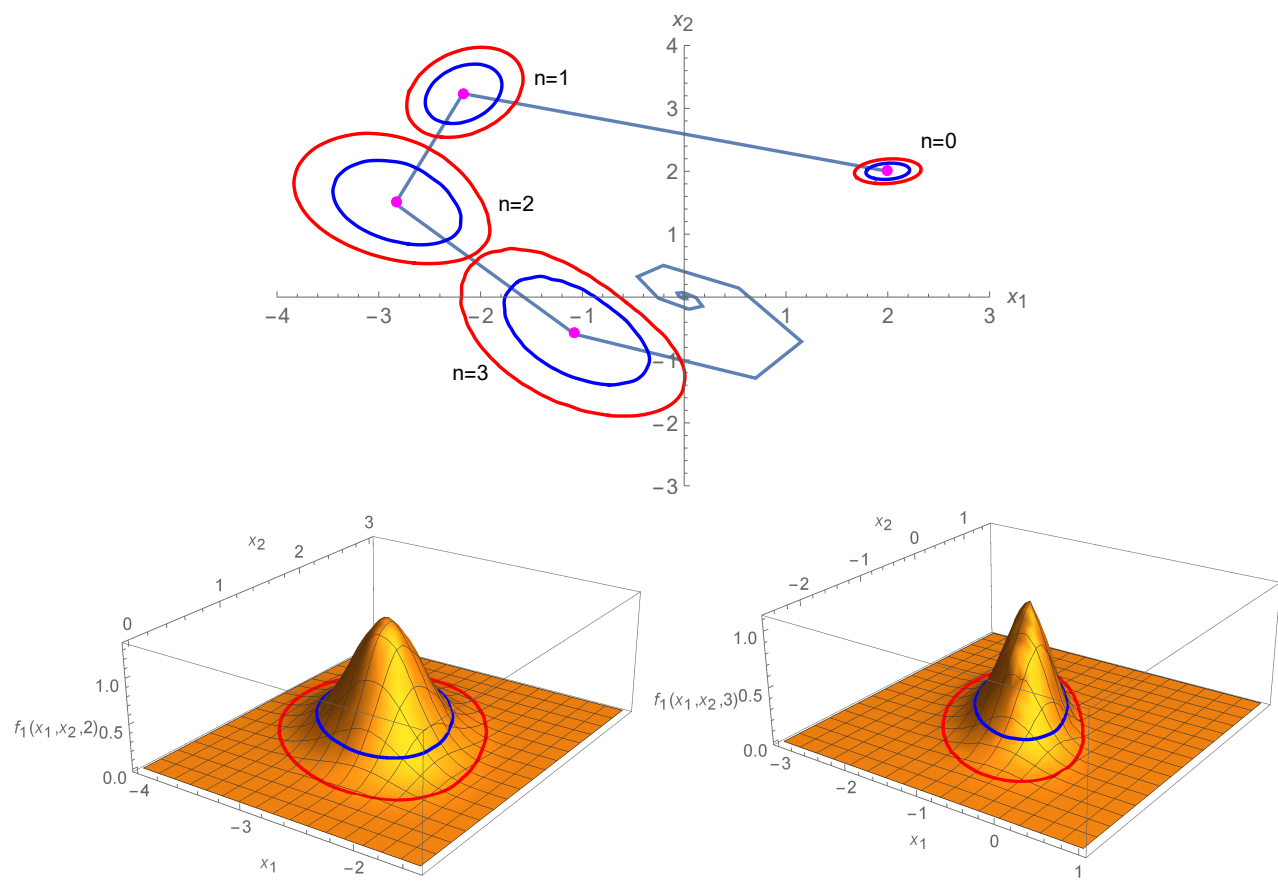

Figure 6.3: Top: Phase portrait of random system of difference equations (6.10) with $m=2$ where random input vector $\mathbf{Z}$ has the Gaussian multivariate distribution given in (6.14)-(6.15). Solid line connects the mean and rings represent confidence regions at $50 \%$ and $90 \%$ confidence levels at every value of $n \in\{0,1,2,3\}$. Bottom left and right: PDFs of solution SP at $n=2$ and $n=3$, respectively, together with the corresponding confidence regions.

\subsection{Conclusions}

Taking advantage of the so-called Random Variable Transformation technique, in this chapter it has been determined the first probability density functions of solution stochastic processes of random autonomous first-order linear systems of differential and difference equations under very general hypotheses (statisti- 
cal dependence among random input data and a wide class of randomness are allowed).

With respect to systems of random differential equations, obtained results are useful to approximate the first probability density function of solution of random nonlinear systems which have been locally approximated using linearization techniques. In the important case of planar systems, that corresponds to $n=2$ in (6.1), it is well-known that there exist several casuistries to classify the $(0,0)$ equilibrium point using phase portrait as stable critical point (sink and spiral sink), unstable critical point (source ans spiral source), saddle point and center. As a significant difference between deterministic and random cases, in the latter scenario each one of those cases corresponds to an event which happens with a specific probability. An important feature of the approach is the quantification of such probability. Although this has been done through an illustrative example, where $(0,0)$ is a stable spiral point, the analysis can be applied for the rest of cases.

From the key information obtained with respect to random difference equations, a nice probabilistic generalization of classical results for the important particular case of planar systems has been provided. This includes the exact quantification of probabilities associated to each possible states to zeroequilibrium point in the case of difference equations. The study comprises the important case of random autonomous linear difference equations of order $m$ as a particular case just taking random coefficient matrix as the so-called companion matrix.

Besides, the results established in this chapter have a great potential regarding applications since many physical models can be properly described by random linear systems of difference equations. In addition, the study of many random autonomous nonlinear models require the application of linearisation to conduct their mathematical analysis. 


\title{
Chapter 7
}

\section{Randomized binary Markov chains}

\begin{abstract}
This chapter is addressed to give a generalization of the classical Markov methodology allowing the treatment of entries of transition matrix and initial condition as random variables instead of deterministic values lying in the interval $[0,1]$. This permits the computation of the first probability density function of solution stochastic process taking advantage of the so-called Random Variable Transformation technique. It will be also computed the distribution of some important quantities related to randomized Markov chains (steady state, hitting times, etc.). All the theoretical results are established under general assumptions and they are illustrated by modelling the diffusion of a technology using real data.
\end{abstract}

\subsection{Introduction}

A SP is a mathematical representation that permits to describe how evolves a phenomenon over the time in a probabilistic manner. Discrete Markov models, also referred to as Markov chains, are a fundamental class of SP where the outcome of an experiment depends only on the outcome of the previous experiment $[7,80]$; this is known as Markov property. This property allows for a considerable reduction of parameters necessary to represent the evolution of a system modelled by such a process. Markov chains are very important and 
widely used to solve problems in a large number of domains such as Operational Research, Computer Science and Distributed Systems, Communication Networks, Biology, Physics, Chemistry, Economics, Finance and Social Sciences, and Medical Decision Making, for instance. In Chapter 8 an example of the applicability of Markov chain in order to model stroke disease will be shown. They are often chosen as suitable tools for modelling very different phenomena because Markov chains are fairly general and adaptable to many contexts $[64,80]$. Moreover, excellent numerical techniques exist for computing statistics associated with them.

This is addressed to give a generalization of classical Markov chains by randomizing entries of transition matrix and initial condition.

Let $\left\{\mathbf{x}_{n}=\left(x_{n}^{1}, x_{n}^{2}\right)^{\top}, n=0,1, \ldots\right\}$ be a Markov chain, where $n$, denotes the cycle or period. Components $x_{n}^{1}$ and $x_{n}^{2}$ lie in the interval $] 0,1[$ and are usually interpreted as percentages or probabilities. Moreover, they satisfy $x_{n}^{1}+x_{n}^{2}=1$ for every $n$. In a Markov chain, the state $\mathbf{x}_{n}$ is determined by the initial condition $\left\{\mathbf{x}_{0}=\left(x_{0}^{1}, x_{0}^{2}\right)^{\top}\right.$ and transition matrix while its asymptotic behaviour only depends on transition matrix. This matrix is a constant matrix whose entries represent the probabilities to change either from one state to another or to remain in the same state between consecutive cycles. Although in practice these entries are usually assumed deterministic, in this contribution this feature is generalized by considering that entries of transition matrix are RVs instead of deterministic constants. Obviously, these RVs are assumed to lie in the interval $[0,1]$ because they must represent probabilities. In Figure 7.1, flow diagram with transitions between states is showed.

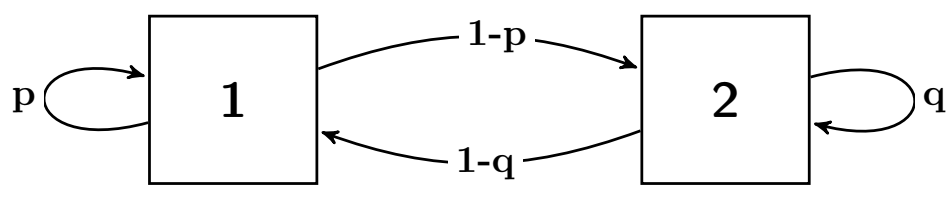

Figure 7.1: Flow diagram to a binary Markov chain.

In the classical context, a Markov binary chain is described as follows

$$
\mathbf{x}_{n+1}=a \mathbf{x}_{n}, \quad n=0,1,2, \ldots, \quad a=\left(\begin{array}{cc}
p & 1-q \\
1-p & q
\end{array}\right)
$$

where $a$ is the transition matrix and $\mathbf{x}_{0}=\left(x_{0}^{1}, x_{0}^{2}\right)^{\top}=\left(x_{0}^{1}, 1-x_{0}^{1}\right)^{\top}$ is the initial condition, i.e., the initial percentage of individuals in each group. 
As indicated above, entries of transition matrix, $p$ and $q$, as well as initial condition, $\mathbf{x}_{0}$, will be considered as RVs. To distinguish RVs from deterministic variables, hereinafter RVs will be written using capital letters. So, randomized binary Markov chain is written as

$$
\begin{aligned}
& \mathbf{X}_{n+1}=A \mathbf{X}_{n}, \quad n=0,1,2, \ldots, \quad A=\left(\begin{array}{cc}
P & 1-Q \\
1-P & Q
\end{array}\right), \\
& \mathbf{X}_{0}=\left(X_{0}^{1}, 1-X_{0}^{1}\right)^{\top},
\end{aligned}
$$

where $X_{0}^{1}, P$ and $Q$ are assumed to be absolutely continuous RVs defined on a common complete probability space $(\Omega, \mathcal{A}, \mathbb{P})$.

The aim of this chapter is to determine the 1-PDF of solution SP to randomized binary Markov chains under general conditions. It will be also computed the distribution of some important quantities related to Markov chains that are very useful in practice. To reach this objective, RVT method will be applied.

The chapter is organized as follows. Section 7.2 is devoted to obtain the 1PDF of solution of a binary Markov chain and its stationary state. Some distributions of interesting quantities of Markov chains will be calculated in Section 7.3. In the last section, Section 7.5, the findings will be applied to model the diffusion of a technology using real data by a binary Markov chain.

\subsection{Probabilistic solution}

This section is divided in two parts. In first subsection the 1-PDF of solution to randomized binary Markov chain (7.1) will be computed under very general assumptions. Second subsection is addressed to determine the PDF of its steady state. These goals will be achieved applying RVT technique.

In order to obtain the PDF of solution to randomized binary Markov chain, solution of problem $(7.1)$ to $n=0,1, \ldots$ is needed and it is given by

$$
\begin{aligned}
\mathbf{X}_{n} & =A^{n} \mathbf{X}_{0} \\
& =\left(\begin{array}{l}
\frac{-1+Q+(-1+P+Q)^{n}\left(1-Q+(-2+P+Q) X_{0}^{1}\right)}{-2+P+Q} \\
\frac{-1+P+(-1+P+Q)^{n}\left(-1+Q-(-2+P+Q) X_{0}^{1}\right)}{-2+P+Q}
\end{array}\right)
\end{aligned}
$$


As $P$ and $Q$ are absolutely continuous RVs, then

$$
\mathbb{P}[\{\omega \in \Omega: P(\omega)+Q(\omega)-2=0\}]=0, \quad \forall \omega \in \Omega .
$$

As a consequence, the denominator of both components of (7.2) is well-defined.

\subsubsection{First probability density function of $\mathrm{X}_{n}$}

As previously indicated, in this subsection the 1-PDF of discrete SP, $\mathbf{X}_{n}$, given by (7.2), will be obtained using RVT method. Since $\mathbf{X}_{n}$ is an SP and RVT method applies to RVs, first fixing the cycle $n$ and defining the following mapping $\mathbf{r}$

$$
\begin{aligned}
& y_{1}=r_{1}\left(x_{0}^{1}, p, q\right)=\frac{-1+q+(-1+p+q)^{n}\left(1-q+(-2+p+q) x_{0}^{1}\right)}{-2+p+q}, \\
& y_{2}=r_{2}\left(x_{0}^{1}, p, q\right)=p \\
& y_{3}=r_{3}\left(x_{0}^{1}, p, q\right)=q .
\end{aligned}
$$

The inverse mapping $\mathbf{s}$ of $\mathbf{r}$ is given by

$$
\begin{aligned}
x_{0}^{1}=s_{1}\left(y_{1}, y_{2}, y_{3}\right) & =\frac{y_{1}\left(-2+y_{2}+y_{3}\right)+\left(-1+y_{3}\right)\left(-1+\left(-1+y_{2}+y_{3}\right)^{n}\right)}{\left(-1+y_{2}+y_{3}\right)^{n}\left(-2+y_{2}+y_{3}\right)}, \\
p=s_{2}\left(y_{1}, y_{2}, y_{3}\right) & =y_{2}, \\
q=s_{3}\left(y_{1}, y_{2}, y_{3}\right) & =y_{3},
\end{aligned}
$$

and the absolute value of the Jacobian

$$
\left|J_{2}\right|=\left|\frac{\partial s_{1}}{\partial y_{1}}\right|=\left|\frac{1}{\left(-1+y_{2}+y_{3}\right)^{n}}\right| \neq 0 .
$$

Therefore, according to Theorem 2.1 the PDF of random vector $\left(Y_{1}, Y_{2}, Y_{3}\right)$ is 


$$
\begin{aligned}
& f_{y_{1}, y_{2}, y_{3}}\left(y_{1}, y_{2}, y_{3}\right) \\
& =f_{X_{0}^{1}, P, Q}\left(\frac{y_{1}\left(-2+y_{2}+y_{3}\right)+\left(-1+y_{3}\right)\left(-1+\left(-1+y_{2}+y_{3}\right)^{n}\right)}{\left(-1+y_{2}+y_{3}\right)^{n}\left(-2+y_{2}+y_{3}\right)}, y_{2}, y_{3}\right) \\
& \quad \times\left|\frac{1}{\left(-1+y_{2}+y_{3}\right)^{n}}\right| .
\end{aligned}
$$

Finally, marginalizing this expression with respect to $P$ and $Q$ and letting $n$ arbitrary, the 1 -PDF of $X_{n}^{1}$ is obtained

$$
\begin{aligned}
& f_{1}^{X^{1}}(x ; n) \\
& =\iint_{\mathcal{D}(P, Q)} f_{X_{0}^{1}, P, Q}\left(\frac{x(-2+p+q)+(-1+q)\left(-1+(-1+p+q)^{n}\right)}{(-1+p+q)^{n}(-2+p+q)}, p, q\right) \\
& \quad \times\left|\frac{1}{(-1+p+q)^{n}}\right| \mathrm{d} q \mathrm{~d} p,
\end{aligned}
$$

where $\mathcal{D}(P, Q)$ stands for the domain of random vector $(P, Q)$.

Now, taking into account that $X_{n}^{2}=1-X_{n}^{1}$ for every $n$, and applying Proposition 2.6 with $a=-1$ and $b=1$, the $1-\mathrm{PDF}$ of $X_{n}^{2}$ is given by

$$
\begin{aligned}
& f_{1}^{X^{2}}(x ; n)=f_{1}^{X^{1}}(1-x ; n) \\
& =\iint_{\mathcal{D}(P, Q)} f_{x_{0}^{1}, P, Q}\left(\frac{(1-x)(-2+p+q)+(-1+q)\left(-1+(-1+p+q)^{n}\right)}{(-1+p+q)^{n}(-2+p+q)}, p, q\right) \\
& \quad \times\left|\frac{1}{(-1+p+q)^{n}}\right| \mathrm{d} q \mathrm{~d} p .
\end{aligned}
$$

One of the most useful applications of these explicit expressions obtained to 1-PDFs, $f_{1}^{X^{i}}(x ; n), i=1,2$, is the direct computation of all one-dimensional statistical moments of $X_{n}^{i}$,

$$
\mathbb{E}\left[\left(X_{n}^{i}\right)^{k}\right]=\int_{\mathbb{R}} x^{k} f_{1}^{X^{i}}(x ; n) \mathrm{d} x, \quad k=1,2, \ldots
$$


Observe that if $k=1$ one obtains the mean of $X_{n}^{i}$ while the variance can be computed using the above moments for $k=1$ and $k=2$, since

$$
\mathbb{V}\left[X_{n}^{i}\right]=\mathbb{E}\left[\left(X_{n}^{i}\right)^{2}\right]-\left(\mathbb{E}\left[X_{n}^{i}\right]\right)^{2} .
$$

\subsubsection{First probability density function of the steady state}

An important issue in dealing with Markov chains is to determine the steady state. From deterministic theory one infers the steady state to randomized Markov chain (7.1)

$$
\mathbf{X}_{\infty}=\left(\begin{array}{c}
\frac{1-Q}{2-P-Q} \\
\frac{1-P}{2-P-Q}
\end{array}\right)
$$

Notice that $\mathbf{X}_{\infty}$ is well-defined because $P$ and $Q$ are absolutely continuous RVs, then $\mathbb{P}[\{\omega \in \Omega: P(\omega)+Q(\omega)-2=0\}]=0$, for all event $\omega \in \Omega$.

Now, the PDF of $\mathbf{X}_{\infty}$ will be obtained. Theorem 2.1 is applied defining the following mapping, $\mathbf{r}$, based on expression (7.5)

$$
\begin{aligned}
& y_{1}=r_{1}(p, q)=\frac{1-q}{2-p-q} \\
& y_{2}=r_{2}(p, q)=q
\end{aligned}
$$

The inverse mapping, $\mathbf{s}$, of $\mathbf{r}$ is given by

$$
\begin{aligned}
& p=s_{1}\left(y_{1}, y_{2}\right)=\frac{-1-y_{1}\left(-2+y_{2}\right)+y_{2}}{y_{1}}, \\
& q=s_{2}\left(y_{1}, y_{2}\right)=y_{2},
\end{aligned}
$$

and the absolute value of the Jacobian of $\mathbf{s}$ is

$$
\left|J_{2}\right|=\left|\frac{\partial s_{1}}{\partial y_{1}}\right|=\left|\frac{1-y_{2}}{y_{1}^{2}}\right| \neq 0
$$


Then, applying Theorem 2.1, the PDF corresponding to the first component of the steady state, $X_{\infty}^{1}$, is

$$
f_{X_{\infty}^{1}}(x)=\int_{\mathcal{D}(Q)} f_{P, Q}\left(\frac{-1-x(2+q)+q}{x}, q\right)\left|\frac{1-q}{x^{2}}\right| \mathrm{d} q .
$$

To compute the PDF corresponding to the second component of the steady state, $X_{\infty}^{2}$, Proposition 2.6 with $a=-1$ and $b=1$ will be applied, taking into account that $X_{\infty}^{2}=1-X_{\infty}^{1}$, obtaining

$$
\begin{aligned}
f_{X_{\infty}^{2}}(x) & =f_{X_{\infty}^{1}}(1-x)= \\
& =\int_{\mathcal{D}(Q)} f_{P, Q}\left(\frac{-1-(1-x)(2+q)+q}{1-x}, q\right)\left|\frac{1-q}{(1-x)^{2}}\right| \mathrm{d} q .
\end{aligned}
$$

\subsection{Relevant probability distributions associated to randomized Markov chains}

In this section the PDF of some useful quantities dealing with randomized discrete Markov chains will be obtained. These quantities are the time until a given proportion of the subpopulation is reached, the probability of first passage and the mean first passage time. In this analysis these quantities extent their deterministic counterpart to random scenario.

\subsubsection{Distribution of time until a given proportion of a subpopulation is reached}

It is useful to know when the percentage of a group in the population will attain a certain level. This motivates the computation of the distribution of the time, $N_{i}, i=1,2$, until a given proportion, $\rho_{i}$, of population of state $i$ is reached. Now, it will be computed $N_{1}$ corresponding to the first subpopulation. Then, let us consider the following relation obtained from the first component of equation (7.2)

$$
\rho_{1}=\frac{-1+q+(-1+p+q)^{n_{1}}\left(1-q+(-2+p+q) x_{0}^{1}\right)}{-2+p+q} .
$$


In order to obtain the 1-PDF, $f_{N_{1}}(n)$, first $n_{1}$ is isolated from equation (7.8) and then capital letter notation is used to random inputs $X_{0}^{1}, P$ and $Q$. This yields

$$
N_{1}=\frac{\log \left(\frac{1-Q+(-2+P+Q) \rho_{1}}{1-Q+(-2+P+Q) X_{0}^{1}}\right)}{\log (-1+P+Q)} .
$$

This RV represents the time until a percentage $\rho_{1}$ of the subpopulation 1 has been reached, so $N_{1}$ must be positive. As

$$
\mathbb{P}[\{\omega \in \Omega: 0<P(\omega)+Q(\omega)-1<1\}]=1,
$$

then

$$
\mathbb{P}\left[\left\{\omega \in \Omega: 0<\frac{1-Q+(-2+P+Q) \rho_{1}}{1-Q+(-2+P+Q) X_{0}^{1}}<1\right\}\right]=1
$$

must hold in order to guarantee positiveness of $N_{1}$. From this condition one can deduce the conditions under which $N_{1}$ can be calculated as

$$
\mathbb{P}\left[\left\{\omega \in \Omega: X_{0}^{1}(\omega)<\rho_{1}<\frac{1-Q(\omega)}{2-P(\omega)-Q(\omega)}\right\}\right]=1,
$$

or

$$
\mathbb{P}\left[\left\{\omega \in \Omega: \frac{1-Q(\omega)}{2-P(\omega)-Q(\omega)}<\rho_{1}<X_{0}^{1}(\omega)\right\}\right]=1 .
$$

These conditions are very intuitive. Indeed, it is easy to check that $X_{n}^{1}$ given by (7.2) is monotone respect to $n$. If it is a monotonically increasing (respect. decreasing) sequence, then condition (7.10) (respect. (7.11)) applies because the proportion $\rho_{1}$ will vary in the interval $\left[X_{0}^{1}(\omega), X_{\infty}^{1}\right]$ (respect. $\left[X_{\infty}^{1}, X_{0}^{1}(\omega)\right]$ ) determined by initial condition and the steady state (7.5).

Using RVT technique with an appropriate mapping $\mathbf{r}$ inspired in (7.9), 


$$
\begin{aligned}
& y_{1}=r_{1}\left(x_{0}^{1}, p, q\right)=\frac{\log \left(\frac{1-q+(-2+p+q) \rho_{1}}{1-q+(-2+p+q) x_{0}^{1}}\right)}{\log (-1+p+q)} \\
& y_{2}=r_{2}\left(x_{0}^{1}, p, q\right)=p \\
& y_{3}=r_{3}\left(x_{0}^{1}, p, q\right)=q
\end{aligned}
$$

it can be proved that the $1-\mathrm{PDF}$ of the time until a percentage, $\rho_{1}$, of the subpopulation 1 has been reached is given by

$$
\begin{aligned}
& f_{N_{1}}(n) \\
& =\iint_{\mathbb{R}^{2}} f_{X_{0}^{1}, P, Q}\left(\frac{\left(1-q+(-1+q)(-1+p+q)^{n}+\rho_{1}(-2+p+q)\right)}{(-1+p+q)^{n}(-2+p+q)}, p, q\right) \\
& \quad \times\left|\frac{\left(-1+q-\rho_{1}(-2+p+q)\right) \log (-1+p+q)}{(-1+p+q)^{n}(-2+p+q)}\right| \mathrm{d} p \mathrm{~d} q .
\end{aligned}
$$

Observe that, for the sake of simplicity the domain of integral (7.12) has not been specified but in practice this domain must be determined taking into account conditions (7.10) or (7.11) depending upon $X_{n}^{1}$ is an increasing or decreasing sequence, respectively.

In an analogous way, one can compute the 1-PDF of the time, $N_{2}$, until a given proportion, $\rho_{2}$, of the subpopulation 2 is reached. This 1-PDF is given by

$$
\begin{aligned}
& f_{N_{2}}(n) \\
& =\iint_{\mathbb{R}^{2}} f_{X_{0}^{1}, P, Q}\left(\frac{\left(-1+p+(-1+q)(-1+p+q)^{n}-\rho_{2}(-2+p+q)\right)}{(-1+p+q)^{n}(-2+p+q)}, p, q\right) \\
& \quad \times\left|\frac{\left(1-p+\rho_{2}(-2+p+q)\right) \log (-1+p+q)}{(-1+p+q)^{n}(-2+p+q)}\right| \mathrm{d} p \mathrm{~d} q .
\end{aligned}
$$




\subsubsection{Distribution of probability of first passage}

In this subsection the 1-PDF of the probability of first passage, $f_{i, j}^{(n)}$, is obtained. $f_{i, j}^{(n)}$ is the probability starting from $i$, that the first visit to state $j$ occurs at time $n$, [80]. If $i=j, f_{i, i}^{(n)}$ is called probability of first return. In addition, from $f_{i, j}^{(n)}, f_{i, j}$ can be calculated, that is, the probability, starting from $i$, that the first visit to state $j$ occurs in a finite time. Probabilities $f_{i, j}^{(n)}$ and $f_{i, j}$ are defined by [80]

$$
f_{i, j}^{(n)}= \begin{cases}P_{i, j}, & \text { if } n=1, \\ \sum_{l \in S \backslash\{j\}} P_{i, l} f_{l, j}^{(n-1)}, & \text { if } n \geq 2,\end{cases}
$$

and

$$
f_{i, j}=P_{i, j}+\sum_{l \in S \backslash\{j\}} P_{i, l} f_{l, j}=\sum_{n=1}^{\infty} f_{i, j}^{(n)},
$$

where $S$ is the state space and $P_{i, j}$ is the probability of moving from state $i$ to state $j$ at the next step.

Here, discrete Markov chains with two states are studied, and then expression (7.13) for each pair $(i, j) \in S \times S$ is given by

$$
\begin{aligned}
& f_{1,1}^{(n)}=\left\{\begin{array}{ll}
P, & \text { if } n=1, \\
(1-P) Q^{n-2}(1-Q), & \text { if } n \geq 2,
\end{array} \quad f_{1,2}^{(n)}=P^{n-1}(1-P), n \geq 1,\right. \\
& f_{2,2}^{(n)}=\left\{\begin{array}{ll}
Q, & \text { if } n=1, \\
(1-Q) P^{n-2}(1-P), & \text { if } n \geq 2,
\end{array} \quad f_{2,1}^{(n)}=Q^{n-1}(1-Q), n \geq 1 .\right.
\end{aligned}
$$

With regard to expression (7.14), for all pair $(i, j) \in S \times S, f_{i, j}=1$. Therefore all states are recurrent and then Markov chain is also recurrent.

Now, it will be obtained the 1-PDF of each expression in (7.15) to each cycle $n$. The PDF of $f_{1,1}^{(n)}$ with $n=1$ is the PDF of RV $P$, it is

$$
f_{1}^{f_{1,1}}(x ; 1)=f_{P}(p) .
$$


In order to obtain the 1-PDF of $f_{1,1}^{(n)}, \forall n \geq 2$, RVT technique is applied. Fixed $n \geq 2$, the following transformation, $\mathbf{r}$, is considered

$$
\begin{aligned}
& x=r_{1}(p, q)=(1-p) q^{n-2}(1-q) \\
& y=r_{2}(p, q)=q .
\end{aligned}
$$

Then, its inverse transformation, s, is given by

$$
\begin{aligned}
& p=s_{1}(x, y)=1+\frac{x y^{2-n}}{-1+y} \\
& q=s_{2}(x, y)=y
\end{aligned}
$$

and the absolute value of the Jacobian is $\left|J_{2}\right|=\left|\frac{y^{2-n}}{-1+y}\right|$ which is nonzero since $Q(\omega) \neq 0$ w.p. 1. Therefore, applying Theorem 2.1, the PDF of random vector $(X, Y)$ is

$$
f_{X, Y}(x, y)=f_{P, Q}\left(1+\frac{x y^{2-n}}{-1+y}, y\right)\left|\frac{y^{2-n}}{-1+y}\right|
$$

Finally, taking $n \geq 2$ arbitrary, the $1-\mathrm{PDF}$ of $f_{1,1}^{(n)}$ is given by

$$
f_{1}^{f_{1,1}}(x ; n)=\int_{\mathcal{D}(Q)} f_{P, Q}\left(1+\frac{x y^{2-n}}{-1+y}, y\right)\left|\frac{y^{2-n}}{-1+y}\right| \mathrm{d} y
$$

Following the same argument, it is easy to check that the $1-\mathrm{PDF}$ of $f_{2,2}^{(n)}$ is given by

$$
f_{1}^{f_{2,2}}(x ; n)= \begin{cases}f_{Q}(q), & \text { if } n=1 \\ \int_{\mathcal{D}(Q)} f_{P, Q}\left(y, 1+\frac{x y^{2-n}}{-1+y}\right)\left|\frac{y^{2-n}}{-1+y}\right| \mathrm{d} y, & \text { if } n \geq 2 .\end{cases}
$$

In the case of the 1 -PDF of $f_{2,1}^{(n)}$ the inverse mapping, for each $n \geq 1$, of function $q^{n-1}(1-q)$ can not be obtained. So, the inverse shall be calculated numerically, using for example the Lagrange-Bürman theorem $[1,16]$. The process to determine the $1-\mathrm{PDF}$ of $f_{1,2}^{(n)}$ is analogous. 


\subsubsection{Distribution of mean first passage time}

For all $i, j \in S$, it can be defined the expected hitting time of state $j$, starting from state $i, m_{i, j}$, using the probability of first passage. That is, as the study is based in a discrete Markov chain, the expectation of the probabilities $f_{i, j}^{(n)}$ is given by

$$
m_{i, j}=\sum_{n=1}^{\infty} n f_{i, j}^{(n)} .
$$

As it is well-known in the literature [80], $m_{i, j}$ can be obtained from the following linear system of equations

$$
m_{i, j}=1+\sum_{k \in S \backslash\{j\}} P_{i, k} m_{k, j}
$$

Then, as there are two possibles states, the different expected times are the following

$$
\begin{array}{ll}
m_{1,1}=\frac{2-P-Q}{1-Q}, & m_{1,2}=\frac{1}{1-P} \\
m_{2,2}=\frac{2-P-Q}{1-P}, & m_{2,1}=\frac{1}{1-Q} .
\end{array}
$$

RVT technique can be applied, using appropriate mappings, in order to obtain the PDF of RVs given in (7.17). Below, obtained results are summarized

- PDF of $m_{1,1}$ :

$$
f_{m_{1,1}}(x)=\int_{\mathcal{D}(Q)} f_{P, Q}(2+x(-1+q)-q, q)|-1+q| \mathrm{d} q .
$$

- PDF of $m_{1,2}$ :

$$
f_{m_{1,2}}(x)=f_{P}\left(\frac{x-1}{x}\right) \frac{1}{x^{2}}
$$


- PDF of $m_{2,1}$ :

$$
f_{m_{2,1}}(x)=f_{Q}\left(\frac{x-1}{x}\right) \frac{1}{x^{2}}
$$

- PDF of $m_{2,2}$ :

$$
f_{m_{2,2}}(x)=\int_{\mathcal{D}(P)} f_{P, Q}(p, 2+p(-1+x)-x)|-1+p| \mathrm{d} p .
$$

\subsection{An application to model the spread of a technology}

In this section an application of the previous theoretical results using real data will be shown. In this example it is considered the number of mobile lines per type of contract in Spain, it is, postpaid or prepaid. Assuming that this situation can be modelled by the binary Markov chain (7.1). For this proposal the data provided by 'Comisión Nacional de los Mercados y la Competencia' [26] will be considered. In Table 7.1, the number of mobile lines per type of contract (postpaid and prepaid) and total number of mobile lines during period 2001-2015 in Spain are collected.

As there are two types of contract, postpaid and prepaid, the state space is $S=\{1,2\}$, and the value 1 to postpaid lines and 2 to prepaid lines will be assigned. As it is assumed that $0<X_{n}^{1}, X_{n}^{2}<1$, the first step is to transform data given in Table 7.1 in proportions. Data in percentages is given in Table 7.2. Denoting these quantities by $Y_{j}^{k}$, where $k \in S$ denotes the corresponding state space and $j \in J=\{0,1, \ldots, 14\}$, corresponds to years $2001,2002, \ldots, 2015$, respectively.

To obtain solution, first it is needed to know the distributions of inputs, so, it is necessary to choose specific probability distributions to random model parameters $X_{0}^{1}, P$ and $Q$. With this aim, in a second step, data collected in Table 7.2 are used in order to assign a reliable probabilistic distributions to random inputs, $P, Q$ and $X_{0}^{1}$, which hereinafter will be assumed independent RVs.

On the one hand, as $X_{0}^{1}$ represents initial proportion of people that has a postpaid mobile line, it will be assumed that $X_{0}^{1}$ has a Uniform distribution with parameters $0 \leq a, b \leq 1$. On the other hand, $P$ and $Q$ are probabilities 


\begin{tabular}{cccccc}
\hline Year & 2001 & 2002 & 2003 & 2004 & 2005 \\
\hline \hline Postpaid & 10384261 & 12657346 & 15592659 & 18555948 & 21980367 \\
\hline Prepaid & 19271468 & 20872651 & 21627180 & 20066634 & 20713465 \\
\hline Total & 29655729 & 33530997 & 37219839 & 38622582 & 42693832 \\
\hline \hline Year & 2006 & 2007 & 2008 & 2009 & 2010 \\
\hline \hline Postpaid & 24794696 & 27657855 & 29310320 & 30187230 & 31420525 \\
\hline Prepaid & 20880959 & 20764615 & 20313019 & 20865463 & 19968892 \\
\hline Total & 45675855 & 48422470 & 49623339 & 51052693 & 51389417 \\
\hline \hline Year & 2011 & 2012 & 2013 & 2014 & 2015 \\
\hline \hline Postpaid & 32220636 & 32850295 & 34409470 & 36199911 & 37618054 \\
\hline Prepaid & 20369871 & 17814804 & 15749219 & 14606340 & 13449515 \\
\hline Total & 52590507 & 50665099 & 50158689 & 50806251 & 51067569 \\
\hline
\end{tabular}

Table 7.1: Number of mobile lines per type of contract (postpaid and prepaid) and total of mobile lines during period 2001-2015 in Spain. Source CNMC [26].

and then they lie between 0 and 1 . Therefore it is considered that both have Beta distributions with parameters $a_{1}, a_{2}>0$ and $b_{1}, b_{2}>0$, respectively.

As $X_{n}^{2}=1-X_{n}^{1}$, the first component of solution SP, $X_{n}^{1}$, will be studied. In order to determine positive parameters $a, b, a_{1}, a_{2}, b_{1}$ and $b_{2}$, it will be minimized the mean square error between data $\left\{Y_{j}^{1}\right\}_{j \in J}$ and the expectation of solution SP, $\left\{X_{j}^{1}\right\}_{j \in J}$, which can be obtained from the 1-PDF given in (7.3). Introducing the probability distributions to the optimization programme

$$
\min _{\substack{0<a, b<1 \\ a_{1}, a_{2}, b_{1}, b_{2}>0}} \sum_{j \in J}\left(Y_{j}^{1}-\mathbb{E}\left[X_{j}^{1}\left(a, b, a_{1}, a_{2}, b_{1}, b_{2}\right)\right]\right)^{2},
$$

the following adjusted parameters are obtained

$$
\begin{array}{ll}
a=0.334388, & b=0.361633 \\
a_{1}=199.218, & a_{2}=2.01382 \\
b_{1}=444.913, & b_{2}=34.0011
\end{array}
$$




\begin{tabular}{cccccc}
\hline Year & 2001 & 2002 & 2003 & 2004 & 2005 \\
\hline \hline Postpaid & 0.350160 & 0.377482 & 0.418934 & 0.480443 & 0.514837 \\
\hline Prepaid & 0.649840 & 0.622518 & 0.581066 & 0.519557 & 0.485163 \\
\hline \hline Year & 2006 & 2007 & 2008 & 2009 & 2010 \\
\hline \hline Postpaid & 0.542843 & 0.571178 & 0.590656 & 0.591296 & 0.61142 \\
\hline Prepaid & 0.457157 & 0.428822 & 0.409344 & 0.408704 & 0.38858 \\
\hline \hline Year & 2011 & 2012 & 2013 & 2014 & 2015 \\
\hline \hline Postpaid & 0.612670 & 0.648381 & 0.686012 & 0.712509 & 0.736633 \\
\hline Prepaid & 0.387330 & 0.351619 & 0.313988 & 0.287491 & 0.263367 \\
\hline
\end{tabular}

Table 7.2: Number of mobile lines in percentage per type of contract (postpaid and prepaid) during period 2001-2015 in Spain. Source CNMC [26].

Once random inputs are determined, one can calculate the 1-PDF of solution of each state and other useful quantities as it has been described in Sections 7.2 and 7.3 .

At this point, it is important to highlight that this approach permits to predict diffusion of mobile lines per type of contract using both punctual predictions (mean) and probabilistic predictions (confidence intervals). This is a main difference against classical approach where only punctual predictions are provided. This distinctive feature is possible because randomization of probabilities of transition matrix and initial conditions. Naturally, this turns out to be a more realistic prediction since sampled data usually contain uncertainty as has been pointed out earlier.

In Figure 7.2, proportion of postpaid mobile lines in Spain in period 2001-2015, $\left\{Y_{j}^{1}\right\}_{j \in J}$, obtained from Table 7.2 is represented in blue points. Expectation of $\left\{X_{j}^{1}\right\}_{j \in J}$ is represented in a solid line. One can observe a good fit between $\left\{Y_{j}^{1}\right\}_{j \in J}$ and expected values $\left\{\mathbb{E}\left[X_{j}^{1}\right]\right\}_{j \in J}$. Also, $75 \%$ and $95 \%$ confidence intervals are plotted. These confidence intervals have been computed as follows. Let us fix a cycle value $\hat{n} \geq 1$ and $\alpha \in(0,1)$, and secondly determine $z_{1}=z_{1}(\hat{n})$ and $z_{2}=z_{2}(\hat{n})$ such that 


$$
\int_{0}^{z_{1}} f_{1}^{X^{1}}(x ; \hat{n}) \mathrm{d} x=\frac{\alpha}{2}=\int_{z_{2}}^{1} f_{1}^{X^{1}}(x ; \hat{n}) \mathrm{d} x .
$$

Then, $(1-\alpha) \times 100 \%$-confidence interval is specified by

$$
\left.1-\alpha=\mathbb{P}\left[\left\{\omega \in \Omega: X_{\hat{n}}^{1}(\omega) \in\left[z_{1}, z_{2}\right)\right]\right\}\right]=\int_{z_{1}}^{z_{2}} f_{1}^{X^{1}}(x ; \hat{n}) \mathrm{d} x .
$$

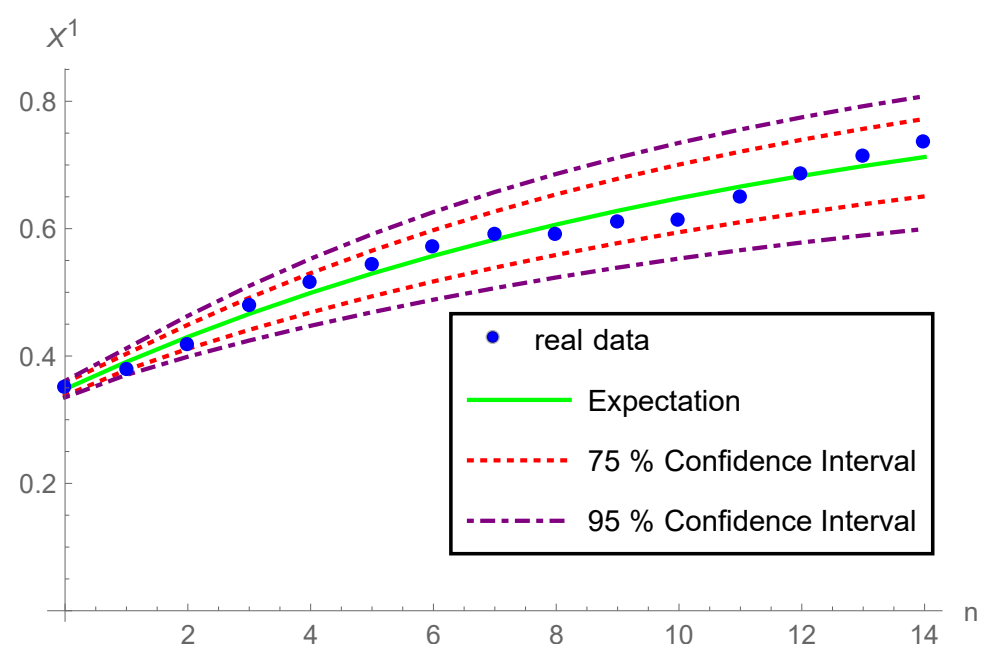

Figure 7.2: Expectation of postpaid mobile lines (solid line) and 75-95\% confidence intervals (dotted lines). Points represent real data.

For each $n \in \mathbb{N}$ fixed, the 1-PDF of $X_{n}^{1}, f_{1}^{X^{1}}(x ; n)$, is determined by (7.3) using the distributions of input RVs, say, $f_{X_{0}^{1}}\left(x_{0}^{1}\right), f_{P}(p)$ and $f_{Q}(q)$. As $X_{0}^{1}, P$ and $Q$ are assumed independent RVs, then $f_{X_{0}^{1}, P, Q}\left(x_{0}^{1}, p, q\right)=f_{X_{0}^{1}}\left(x_{0}^{1}\right) f_{P}(p) f_{Q}(q)$. In Figure 7.3-left, the 1-PDF of $X_{n}^{1}$ is plotted at different fixed cycles: $n=1$, in blue, $n=6$ in red, $n=11$ in gray, etc. One can observe that these 1PDFs, as $n \rightarrow \infty$, tend to the PDF of the steady state, $f_{X_{\infty}^{1}}(x)$, calculated by expression (7.6) and represented in black colour. Figure 7.3-left shows that $f_{1}^{X^{1}}(x ; 70)$ practically match with $f_{X_{\infty}^{1}}(x)$. The 1 -PDF of $X_{n}^{2}$ is calculated using transformation from $f_{1}^{X^{1}}(x ; n)$ given by (7.4). The analogous transformation (7.7) applied to $f_{X_{\infty}^{1}}(x)$ is used to determine de PDF for the steady state of $X_{n}^{2}, f_{X_{\infty}^{2}}(x)$. Results related to prepaid lines are shown in Figure 7.3-right. As it is expected, one can observe the symmetry of results in both subfigures. 

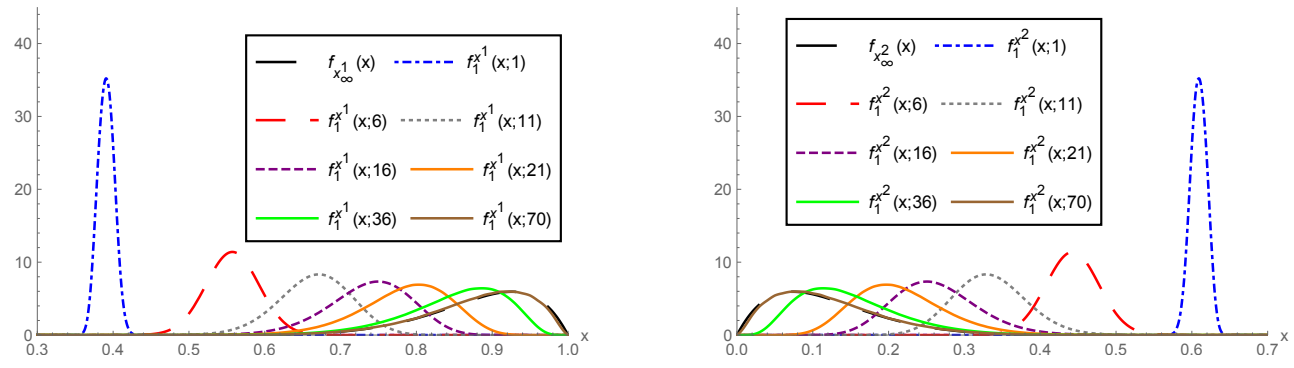

Figure 7.3: Left: Plot of $f_{1}^{X^{1}}(x ; n)$ given by $(7.3)$ for several cycles and $f_{X_{\infty}^{1}}(x)$ given by (7.6). Right: Plot of $f_{1}^{X^{2}}(x ; n)$ given by (7.4) for several cycles and $f_{X_{\infty}^{2}}(x)$ given by (7.7).

Figure 7.4 shows the distribution of time until a given proportion of population, $\rho_{1}$, possesses postpaid line mobile. This PDF is determined by the equation (7.12) and it is represented for different values of these proportions, $\rho_{1}$. As is it expected, when the value of $\rho_{1}$ increases, the maximum of the corresponding PDFs moves to the right. In Table 7.3, the mean and the standard deviation of $f_{N_{1}}(n)$ at several values of $\rho_{1}$ are given. For example, from Figure 7.4, the distribution of time until a $40 \%$ of population has a postpaid mobile line reaches its maximum near 1 and it is narrow. Notice that the expectation and the standard deviation in Table 7.3 for $\rho_{1}=0.4$ is in agreement with the representation of the corresponding $\mathrm{PDF}$ in Figure 7.4. One can observe, for each $\rho_{1}$ fixed, that PDF representations in Figure 7.4 are also in agreement with corresponding values of Table 7.3.

\begin{tabular}{cccccc}
\hline$\rho_{1}$ & 0.4 & 0.45 & 0.5 & 0.55 & 0.6 \\
\hline \hline $\mathbb{E}\left[f_{N_{1}}\right]$ & 1.275 & 2.648 & 4.207 & 6.0127 & 8.149 \\
\hline$\sigma\left[f_{N_{1}}\right]$ & 0.332 & 0.629 & 1.044 & 1.638 & 2.506 \\
\hline
\end{tabular}

Table 7.3: Expectation and standard deviation of $f_{N_{1}}(n)$ (given by (7.12)) for several values of $\rho_{1}$.

The 1-PDF of the probability of first return $f_{1,1}^{(n)}$ given by (7.16) at cycle $n=1$, it is, the probability of remaining at state 1 , at cycle 1 , starting from state 1 is represented in Figure 7.5. From representation of $1-\mathrm{PDF} f_{1}^{f_{1,1}}(x ; 1)$, one can conclude that if you have a postpaid line now, you will probably have a 


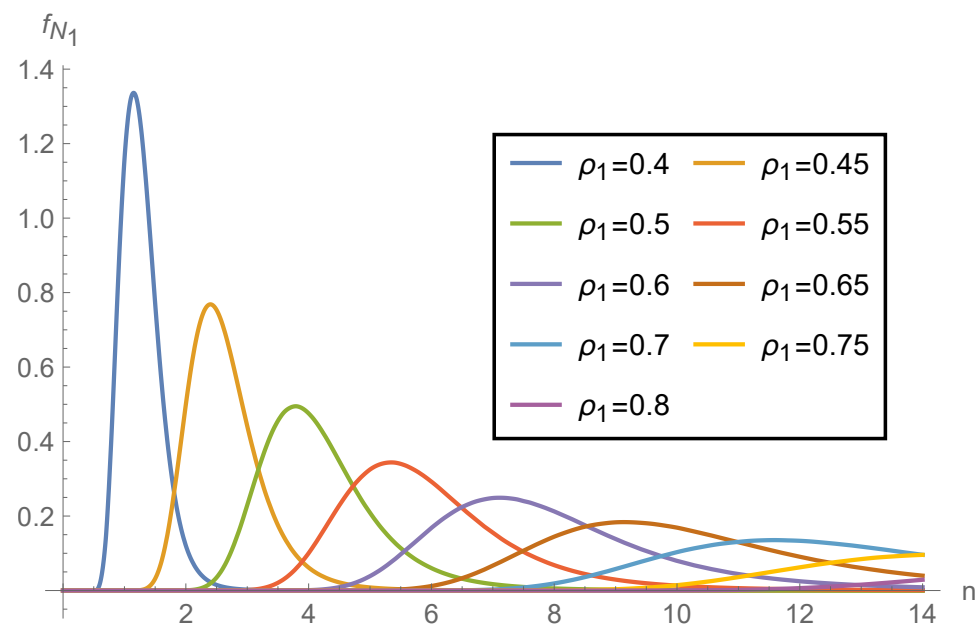

Figure 7.4: Plot of $f_{N_{1}}(n)$ given by (7.12) for several values of $\rho_{1}$.

postpaid line next year. This is in agreement with the expected value of $P$, $\mathbb{E}[P]=0.98$.

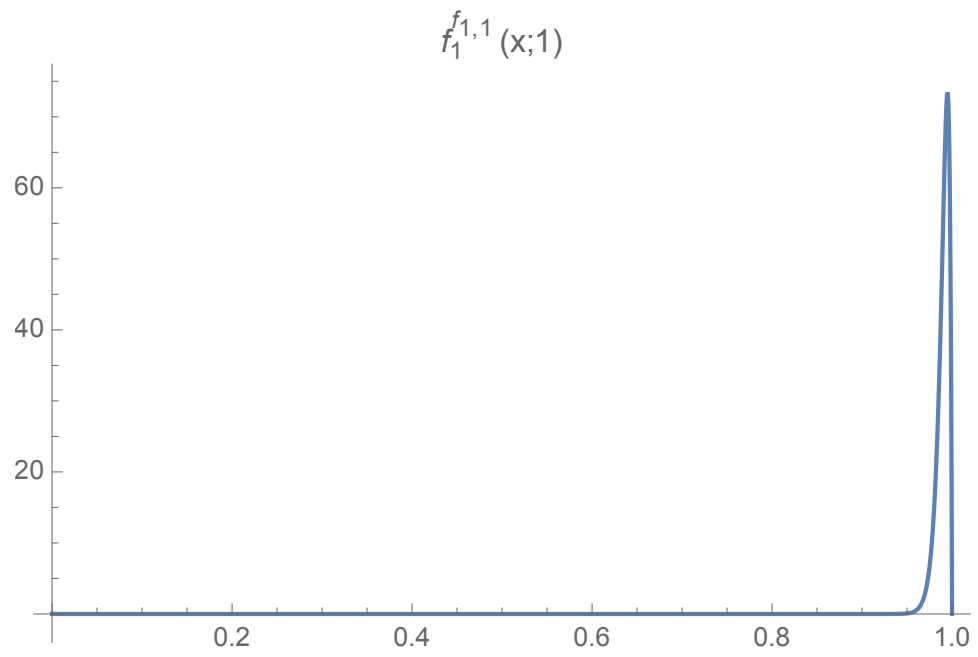

Figure 7.5: $1-\mathrm{PDF}$ of the probability of first return $f_{1,1}^{(n)}$ given by $(7.16)$ at $n=1$. 
In Figure 7.6-left, the 1-PDF of the probability of first passage $f_{1,1}^{(n)}$ at the rest of cycles, $n \geq 2$, is plotted. Results are in agreement with Figure 7.5 because a little proportion of population change from postpaid line to prepaid line. In Figure 7.6-right expectation plus/minus standard deviation for $n \geq 2$ are plotted.
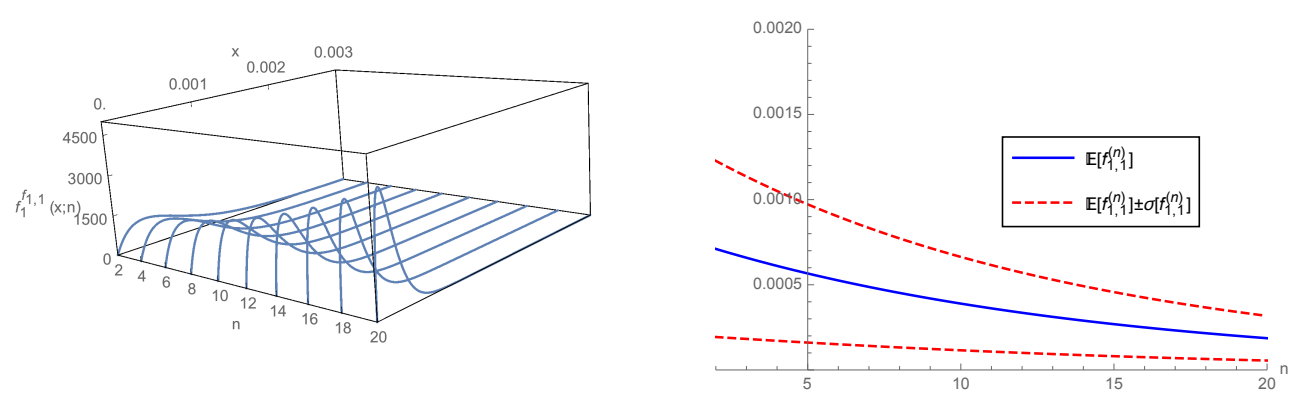

Figure 7.6: Left: $1-\mathrm{PDF}$ of the probability of first return $f_{1,1}^{(n)}$ given by (7.16) at cycles $n \in\{2,4, \ldots, 20\}$ (Corresponding to the solid lines). Right: Expectation of $f_{1,1}^{(n)}$ plus/minus standard deviation for $n \geq 2$.

As it is indicated in the Subsection 7.3.2, the 1-PDF of first passage $f_{2,1}^{(n)}$ is calculated numerically using Lagrange-Bürman Theorem. Results for the 1PDF of the probability of first passage $f_{2,1}^{(n)}$ are showed in the upper part of Figure 7.7 (top). Due to scale in vertical axis, for the sake of clarity in the presentation, it has been split in two plots. In Figure 7.7 (bottom), expectation plus/minus standard deviation for $n \geq 2$ are plotted.

Finally, in Figure 7.8 the results for the PDF of the mean first passage time between two states, $m_{i, j}$, given by (7.18)-(7.21) are shown. In Table 7.8 expectation and standard deviation of each $m_{i, j}$ are provided. In both, figure and table, one observes that expected mean time passage $m_{2,1}$ is approximately 14 . The expected mean time passage $m_{1,1}$ is approximately 1 , in accordance with previous comments. 

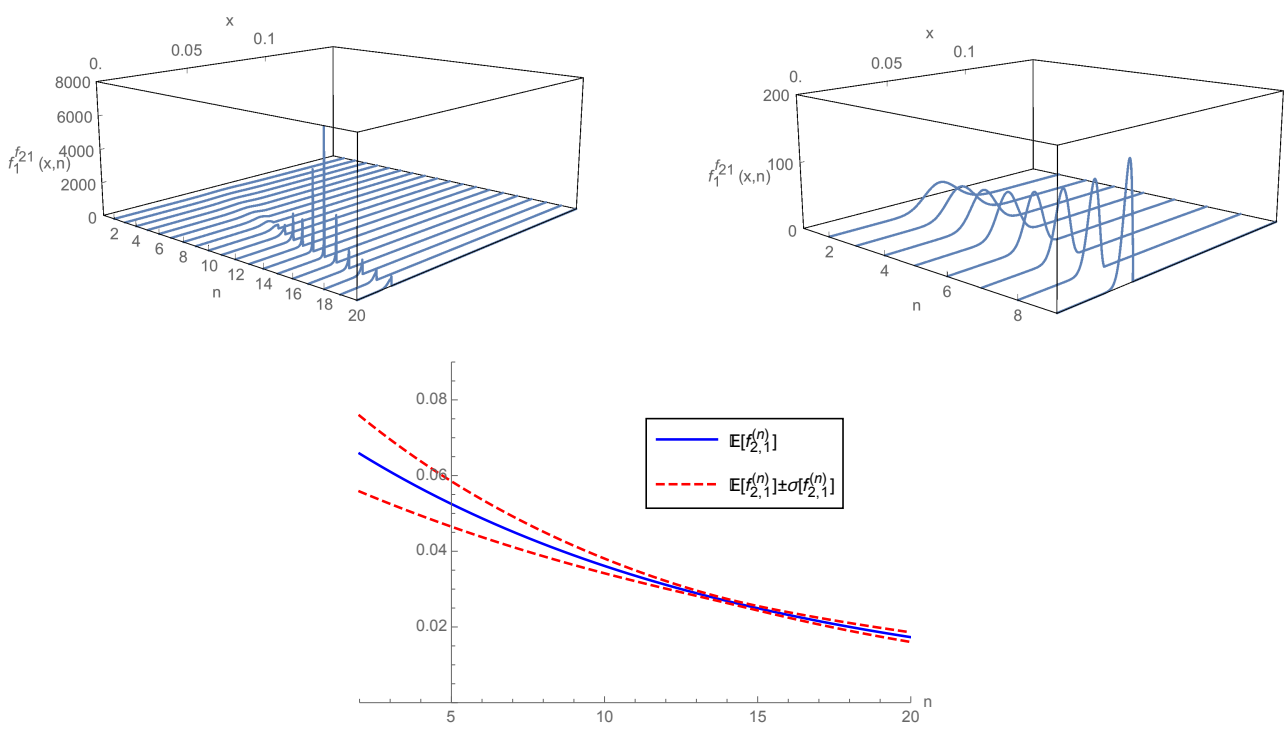

Figure 7.7: Top-left: 1 -PDF of the probability of first return $f_{2,1}^{(n)}$ at cycles $n \in\{2,3, \ldots$, $20\}$. Top-right: Zoom of 1-PDF of the probability of first return $f_{2,1}^{(n)}$ at cycles $n \in\{2,3$, $\ldots, 9\}$. Bottom: Expectation of $f_{2,1}^{(n)}$ plus/minus standard deviation for $n \geq 2$.

\begin{tabular}{ccccc}
\hline & $m_{1,1}$ & $m_{1,2}$ & $m_{2,1}$ & $m_{2,2}$ \\
\hline \hline $\mathbb{E}[\cdot]$ & 1.14493 & 197.325 & 14.4818 & 14.9909 \\
\hline$\sigma[\cdot]$ & 0.105755 & 447.492 & 2.47 & 29.5575 \\
\hline
\end{tabular}

Table 7.4: Expectation and standard deviation of the expected hitting time of state $j$ starting from state $i, m_{i, j}$.

\subsection{Conclusions}

In this chapter it has been provided a full probabilistic description of solution of a random binary Markov chain under very general assumptions on random inputs. These random inputs are the probabilities of transition matrix and initial condition. By means of randomization of these probabilities, the approach provides a generalization of relevant results to classical binary Markov chains. The aforementioned full probabilistic description has been made through the first probability density function of the discrete solution stochastic process and 

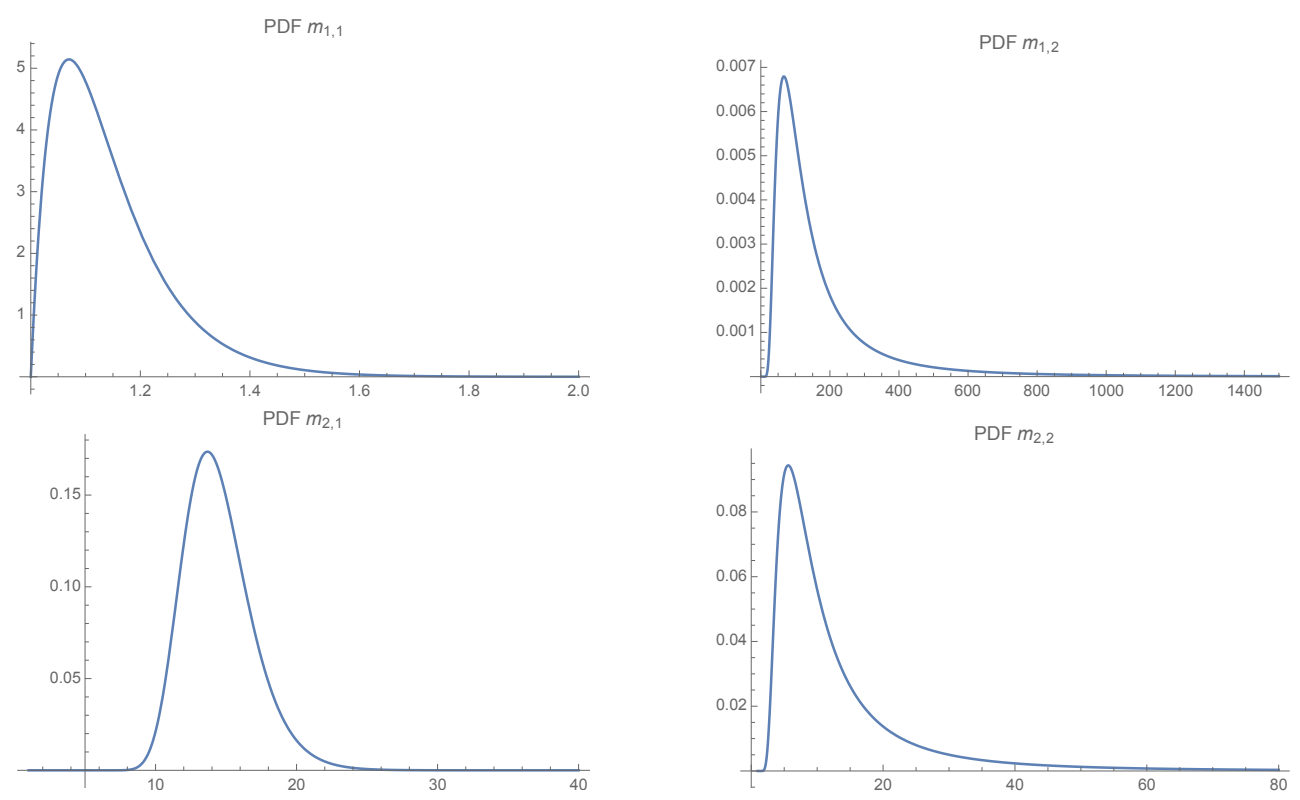

Figure 7.8: PDF of the expected hitting time of state $j$ starting from state $i, m_{i, j}$, given by $(7.18)-(7.21)$.

the probability density function associated to the steady state. Furthermore, the probability density function of a key time having specific interpretation in practice has been determined. Other quantities of great interest in the deterministic context of Markov chains, like the probability of first passage time and the mean first passage time have been randomized using this approach. Then, a full probabilistic description of these quantities have been established. A key mathematical tool to conduct the analysis has been the application of Random Variable Transformation technique. Finally the findings have been illustrated to model the percentage of mobile lines per contract type (postpaid and prepaid) in Spain using real data. This data are well-modelled through a random binary Markov chain. 



\section{Chapter 8}

\section{Probabilistic solution of a Markov chain to model stroke disease}

Classical Markov models are defined through a matrix whose columns (or rows) are deterministic values representing transition probabilities. However, in practice these quantities could often not be known in a deterministic manner, therefore, it is more realistic to consider them as random variables. Following this approach, this chapter is aimed to give a technical generalization of classical Markov methodology in order to improve modelling of stroke disease when dealing with real data. With this goal, entries of transition matrix of a Markov chain with three states (susceptible, reliant and deceased) that has been previously proposed to model stroke disease will be randomized. This permits the computation of the first probability density function of solution stochastic process taking advantage of the so-called Random Variable Transformation technique. Afterwards, punctual and probabilistic predictions are computed from the first probability density function. In addition, probability density functions of time instants until a certain proportion of total population remains susceptible, reliant and deceased are also computed. The study is completed showing the usefulness of the computational approach to determine, from a probabilistic point of view, key quantities in medical decision making, such as cost-effectiveness ratio. 


\subsection{Introduction}

Discrete Markov SPs or discrete Markov chains are often applied to model dynamics of medical events over evenly spaced times, $n=0,1,2, \ldots$, usually referred to as periods or cycles. In particular, these kind of SPs have been considered for different purposes. For example, to built and simulate models for chronic illnesses $[12,84]$, to analyse data of hospital infection [28], to provide predictions based on random-effects Markov models applied to multiple sclerosis progression [62], to calculate the prevalence of certain diseases and to perform budget impact analysis [63], to model human papilloma virus [58], etc. In these models individuals are classified in several disjoint classes or states. The evolution of percentage (or number of individuals) in each cycle $n$ is determined by the initial distribution of individuals and a stochastic matrix, usually termed transition matrix. An important assumption of standard Markov models is that all states make up a closed system. This means that any individuals can neither leave nor join the system, hence having a constant population size over time. This hypothesis holds in clinical context where doctors and public health authorities are often interested in the evolution of patient groups in controlled studies over time. In the case of discrete homogeneous Markov SPs, transition matrix is a constant matrix whose entries represent probabilities to change either from one state to another or to remain in the same state between two consecutive cycles. When these probabilities depend upon time, the SPs are termed non-homogeneous time discrete Markov chains. In both cases, entries of transition matrix are assumed to be deterministic quantities (numbers or functions, respectively). In this chapter, this feature is generalized for discrete homogeneous Markov chains by considering that entries in transition matrix could be RVs rather than deterministic constants. Naturally, RVs are assumed to take values in the interval $[0,1]$, thus representing probabilities for every realization of such RVs. In this manner, more flexibility is allowed when probabilities are assigned. Throughout this chapter, this approach will be considered to generalize stroke disease model proposed in [64]. It is important to point out that the application of this approach is not limited to stroke disease model presented later but is also valid for modelling any disease via time discrete homogeneous Markov chains.

According to [64], stroke disease can be modelled via a Markov chain considering the three following states, Susceptible $(S)$, Reliant $(R)$ and Deceased $(D)$. In Figure 8.1 influence or flow diagram associated to Markov model is shown. In this graphical representation, transitions among states have been included. One observes that, apart from remaining in each state, the possible transitions between states are $S \rightarrow R, S \rightarrow D$ and $R \rightarrow D$. Thus, reliant population 


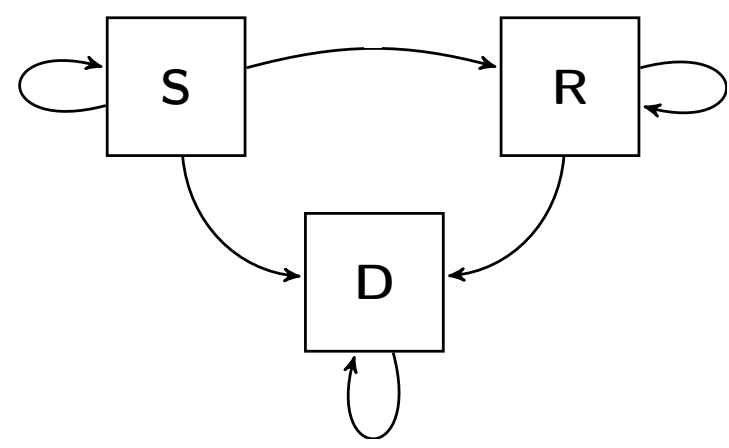

Figure 8.1: Flow diagram for Markov model (8.1)-(8.2). $S, R$ and $D$ stand for Susceptible $(S)$, Reliant $(R)$ and Deceased $(D)$.

cannot recover from the disease. Obviously, state $D$ is an absorbing state. In this study susceptible individuals make up a population at risk, i.e., they have certain pathologies (hypertension, cholesterol, etc.) that may conduct to suffer a stroke. Therefore, as it shall been sawn below, the model involves a relative risk.

In [64], Markov model is formulated as follows

$$
\left(\begin{array}{c}
S_{n+1} \\
R_{n+1} \\
D_{n+1}
\end{array}\right)=T\left(\begin{array}{c}
S_{n} \\
R_{n} \\
D_{n}
\end{array}\right), \quad\left(S_{0}, R_{0}, D_{0}\right)^{\top}=\left(s_{0}, r_{0}, d_{0}\right)^{\top}, \quad n=0,1,2, \ldots,
$$

where $S_{n}, R_{n}$ and $D_{n}$ are the proportion of susceptible, reliant and deceased subpopulations in cycle $n$, respectively. As a matter of fact in dealing with markovian models, it will be assumed that $S_{n}+R_{n}+D_{n}=1$ for each $n$. As it is plausible from a practical standpoint, it is assumed that initially there are no deaths, hence the initial cohort corresponds with the deterministic vector $\left(s_{0}, r_{0}, 0\right)^{\top}, s_{0}+r_{0}=1$. Otherwise, subsequent analysis follows analogously. Moreover, according to results given in [64], it shall been assumed that transition matrix $T$ is given by

$$
T=\left(\begin{array}{ccc}
\mathrm{e}^{-t_{1} r r}+\mathrm{e}^{-\left(t_{2}+t_{3}(r r-1)\right)}-1 & 0 & 0 \\
1-\mathrm{e}^{-t_{1} r r} & 1-p & 0 \\
1-\mathrm{e}^{-\left(t_{2}+t_{3}(r r-1)\right)} & p & 1
\end{array}\right)
$$


where

- $r r$ is the relative risk of suffering a stroke,

- $t_{1}$ is the non-mortal stroke rate,

- $t_{2}$ is the deceased rate due to any cause,

- $t_{3}$ is the stroke death rate and,

- $p$ is the probability of transition $R \rightarrow D$,

rates $t_{1}, t_{2}$ and $t_{3}$ are given for a general population. For the sake of clarity, the construction of transition matrix, $T$, in connection with [64, Table 3] and the meaning of parameters previously introduced is explained. Element $(2,1)$ of matrix $T, T_{21}$, represents the probability of suffering a non-mortal stroke in cycle $n+1$ given that individual was susceptible in cycle $n(S \rightarrow R)$. The probability of having a stroke is given by 1 minus the probability of does not have it, being these kind of probabilities usually modelled by an exponential decay. In [64, Table 3] this probability is given by $1-\mathrm{e}^{-(\text {'non-mortal stroke rate') }}$. The 'non-mortal stroke rate' is given by $t_{1}$ and taking into account that it is population under risk, this leads to term $t_{1} r r$. $T_{31}$ denotes the probability of transition $S \rightarrow D$. In [64, Table 3], this probability is given by $1-\mathrm{e}^{- \text {('death rate') }}$. Observe that the 'death rate' involved in $T_{31}$ is given by $\left(t_{2}+t_{3}(r r-1)\right)=$ $t_{2}-t_{3}+t_{3} r r$, that is, the non-stroke death rate for a general population, $t_{2}-t_{3}$, adding the term corresponding to stroke death rate for population under risk given by $t_{3} r r$.

At this point it is important to remark that parameter $r r$ that is termed relative risk, in the context of medicine is a positive number [66, 97].

Remark 8.1 From a mathematical standpoint parameters $t_{1}, t_{2}, t_{3}$ and $r r$ must satisfy condition $0<T_{21}+T_{31}<1$. This guarantees that $\left.T_{11} \in\right] 0,1[$. As in practice, the rates $t_{1}, t_{2}$ and $t_{3}$ are small, former condition holds.

As it has been pointed out previously, a major difference with respect to contribution [64] is that some model parameters, namely, $t_{2}, r r$ and $p$, involved in transition matrix $T$ are assumed to be absolutely continuous RVs rather than deterministic constants. Hereinafter, as usual in Probability Theory, capital letters will be used to highlight this difference. Hence, the following identifications, $t_{2} \Rightarrow T_{2}, r r \Rightarrow R R$ and $p \Rightarrow P$, will be used (see expression (8.2)). This decision is motivated inasmuch as, in practice, death rate due to any cause, 
$T_{2}$, is not known in a deterministic way and relative risk of suffering a stroke, $R R$, varies among physical characteristic of individuals being this variation non-deterministic. Regarding parameter $P$, which represents a probability, it can be described by a RV whose domain is contained in interval ]0,1[, allowing for more flexibility throughout the study. In the following, triplet $(\Omega, \mathcal{A}, \mathbb{P})$ will denote the common complete probability space where RVs $T_{2}, R R$ and $P$ are defined.

Summarizing, the model under study is

$$
\left(\begin{array}{c}
S_{n+1} \\
R_{n+1} \\
D_{n+1}
\end{array}\right)=T\left(\begin{array}{c}
S_{n} \\
R_{n} \\
D_{n}
\end{array}\right), \quad\left(S_{0}, R_{0}, D_{0}\right)^{\top}=\left(s_{0}, r_{0}, 0\right)^{\top}, \quad n=0,1,2, \ldots,
$$

where transition matrix is given by

$$
T=\left(\begin{array}{ccc}
\mathrm{e}^{-t_{1} R R}+\mathrm{e}^{-\left(T_{2}+t_{3}(R R-1)\right)}-1 & 0 & 0 \\
1-\mathrm{e}^{-t_{1} R R} & 1-P & 0 \\
1-\mathrm{e}^{-\left(T_{2}+t_{3}(R R-1)\right)} & P & 1
\end{array}\right)
$$

In connection with Remark 8.1 and, in random context, to guarantee positiveness of entry $T_{11}(\omega)$ of random matrix (8.2), it must be imposed that RVs $R R$ and $T_{2}$ satisfy the following condition

$$
\mathbb{P}\left[0<T_{21}(\omega)+T_{31}(\omega)<1\right]=1, \forall \omega \in \Omega
$$

where

$$
T_{21}(\omega)+T_{31}(\omega)=2-\mathrm{e}^{-t_{1} R R(\omega)}-\mathrm{e}^{-\left(T_{2}(\omega)+t_{3}(R R(\omega)-1)\right)}
$$

To conduct the study, the so-called RVT method will be used. This technique has been successfully applied in previous contributions related to epidemiological models, some examples include $[15,18,34,35,54]$. RVT method allows to obtain the 1-PDF of solution SPs, $S_{n}, R_{n}, D_{n}$, to model (8.1)-(8.2). Additionally, PDFs of times until a given proportion of population remains susceptible, reliant and deceased, respectively will be computed. Finally, the PDF of costeffectiveness ratio will be also computed taking advantage of RVT technique. This is a key quantity in medical decision making. 
The chapter is organized as follows. Section 8.2 is devoted to provide a probabilistic solution of randomized Markov model (8.1)-(8.2) by means of the first probability density function for each subpopulation, susceptible, reliant and deceased. For the sake of clarity this section has been divided into two subsections. First, in Subsection 8.2.1 the main statistical properties, such as, mean, variance and confidence intervals, are computed. Secondly, Subsection 8.2.2 is addressed to obtain the probabilistic distribution until a given proportion of population remains susceptible. In Section 8.3 a cost-effectiveness analysis is performed. In Section 8.4, all theoretical results developed throughout Sections 8.2 and 8.3 are applied to simulate stroke disease taking particular distributions for random input data that are in agreement with the extant literature. In Section 5.4, it will be discussed the main findings.

\subsection{Probabilistic solution}

As it has been said previously, the goal of this section is to obtain the 1PDF of the number of susceptibles, reliants and deceaseds, which are the components of solution SP of random IVP (8.1)-(8.2). This will be done in terms of random input data. For the sake of generality as it has been indicated previously, throughout this subsection $R R, T_{2}$ and $P$ are assumed to be absolutely continuous dependent RVs, defined on a common probability space $(\Omega, \mathcal{A}, \mathbb{P})$, with joint $\mathrm{PDF} f_{R R, T_{2}, P}\left(r r, t_{2}, p\right)$ defined on a domain, say $\mathcal{D}\left(R R, T_{2}, P\right)$. It generalizes the case where $R R, T_{2}$ and $P$ are assumed to be independent RVs with PDFs $f_{R R}(r r), f_{T_{2}}\left(t_{2}\right)$ and $f_{P}(p)$, since in that case $f_{R R, T_{2}, P}\left(r r, t_{2}, p\right)=f_{R R}(r r) f_{T_{2}}\left(t_{2}\right) f_{P}(p)$. Although less general, independence is a hypothesis usually embraced in probabilistic applications.

As it is well known, the solution of IVP (8.1)-(8.2) is

$$
\left(\begin{array}{c}
S_{n} \\
R_{n} \\
D_{n}
\end{array}\right)=T^{n}\left(\begin{array}{c}
s_{0} \\
r_{0} \\
0
\end{array}\right) .
$$

To conduct the study it is convenient to recast entries of transition matrix $T$ as follows

$$
T=\left(\begin{array}{ccc}
1-K-Q & 0 & 0 \\
K & 1-P & 0 \\
Q & P & 1
\end{array}\right)
$$


where $K=1-\mathrm{e}^{-t_{1} R R}$ and $Q=1-\mathrm{e}^{-\left(T_{2}+t_{3}(R R-1)\right)}$. Then, developing the right-hand side of (8.4) one gets

$$
\begin{aligned}
& S_{n}=(1-K-Q)^{n} s_{0} \\
& R_{n}=\frac{(1-K-Q)^{n} K s_{0}-(1-P)^{n}\left(r_{0}(-P+Q+K)+K s_{0}\right)}{P-Q-K} \\
& D_{n}=r_{0}\left(1-(1-P)^{n}\right)+\frac{\left((P-Q)\left(1-(1-Q-K)^{n}\right)+\left(-1+(1-P)^{n}\right) K\right) s_{0}}{P-Q-K}
\end{aligned}
$$

Notice that, as $P, Q$ and $K$ are absolutely continuous RVs, the denominator of second and third components of expression (8.5) are non-zero w.p. 1. Taking into account $S_{n}+R_{n}+D_{n}=1$ for each $n$, it is enough to determine the 1-PDF of susceptible and reliant subpopulations, since from them, it is straightforward to obtain the 1-PDF of deceased subpopulation.

This goal will be achieved by applying RVT method twice. First, the joint PDF, $f_{S_{n}, R_{n}, P}(s, r, p)$, of random vector $\left(S_{n}, R_{n}, P\right)$ will be computed from joint PDF, $f_{K, Q, P}(k, q, p)$, of $(K, Q, P)$, and secondly, the joint PDF, $f_{K, Q, P}(k, q, p)$, of $(K, Q, P)$ will be computed from joint $\mathrm{PDF}, f_{R R, T_{2}, P}\left(r r, t_{2}, p\right)$, of random input data $\left(R R, T_{2}, P\right)$.

Now, fixing the cycle $n$, RVT method, Theorem 2.1, is applied with the following identification

$$
\mathbf{X}=(K, Q, P), \quad \mathbf{Y}=\left(Y_{1}, Y_{2}, Y_{3}\right), \quad \mathbf{Y}=\mathbf{r}(\mathbf{X})
$$

being the mapping $\mathbf{r}: \mathbb{R}^{3} \rightarrow \mathbb{R}^{3}$

$$
\begin{aligned}
& y_{1}=r_{1}(k, q, p)=(1-k-q)^{n} s_{0}, \\
& y_{2}=r_{2}(k, q, p)=\frac{(1-k-q)^{n} k s_{0}-(1-p)^{n}\left(r_{0}(-p+q+k)+k s_{0}\right)}{p-q-k} \\
& y_{3}=r_{3}(k, q, p)=p .
\end{aligned}
$$


Isolating $k, q$ and $p$ one gets

$$
\begin{aligned}
k= & \frac{\left(-1+y_{3}+\left(\frac{y_{1}}{s_{0}}\right)^{\frac{1}{n}}\right)\left(r_{0}\left(1-y_{3}\right)^{n}-y_{2}\right)}{\left(1-y_{3}\right)^{n} s_{0}-y_{1}}, \\
q= & \frac{y_{1}-y_{1}\left(\frac{y_{1}}{s_{0}}\right)^{\frac{1}{n}}+\left(-s_{0}+\left(r_{0}+s_{0}\right)\left(\frac{y_{1}}{s_{0}}\right)^{\frac{1}{n}}+r_{0}\left(-1+y_{3}\right)\right)\left(1-y_{3}\right)^{n}}{y_{1}-s_{0}\left(1-y_{3}\right)^{n}} \\
p= & -\frac{y_{2}\left(-1+\left(\frac{y_{1}}{s_{0}}\right)^{\frac{1}{n}}+y_{3}\right)}{y_{1}-s_{0}\left(1-y_{3}\right)^{n}},
\end{aligned}
$$

For the sake of clarity, hereinafter $m_{1}:=k$ and $m_{2}:=q$ will be considered.

Notice that the Jacobian of the inverse mapping of $\mathbf{r}$ is

$$
J_{3}=\frac{\left(\frac{y_{1}}{s_{0}}\right)^{1 / n}\left(-1+\left(\frac{y_{1}}{s_{0}}\right)^{1 / n}+y_{3}\right)}{n y_{1}\left(y_{1}-s_{0}\left(1-y_{3}\right)^{n}\right)} \neq 0, \quad \text { a.s. }
$$

Then, taking into account (2.16)-(2.17), the joint PDF of random vector $\left(Y_{1}, Y_{2}, Y_{3}\right)=\left(S_{n}, R_{n}, P\right)$ is given by

$$
f_{S_{n}, R_{n}, P}\left(y_{1}, y_{2}, y_{3}\right)=f_{K, Q, P}\left(m_{1}, m_{2}, y_{3}\right)\left|\frac{\left(\frac{y_{1}}{s_{0}}\right)^{1 / n}\left(-1+\left(\frac{y_{1}}{s_{0}}\right)^{1 / n}+y_{3}\right)}{n y_{1}\left(y_{1}-s_{0}\left(1-y_{3}\right)^{n}\right)}\right| .
$$

Now, Theorem 2.1 is used again with the following identification

$$
\mathbf{X}=\hat{\mathbf{X}}=\left(R R, T_{2}, P\right), \quad \mathbf{Y}=\hat{\mathbf{Y}}=\left(\hat{Y}_{1}, \hat{Y}_{2}, \hat{Y}_{3}\right), \quad \hat{\mathbf{Y}}=\mathbf{r}(\hat{\mathbf{X}}),
$$

being the mapping $\mathbf{r}: \mathbb{R}^{3} \rightarrow \mathbb{R}^{3}$ 


$$
\hat{y}_{1}=1-\mathrm{e}^{-t_{1} r r}, \quad \hat{y}_{2}=1-\mathrm{e}^{-\left(t_{2}+t_{3}(r r-1)\right)}, \quad \hat{y}_{3}=p
$$

and isolating $r r, t_{2}$ and $p$, one gets

$$
r r=\frac{-\log \left(1-\hat{y}_{1}\right)}{t_{1}}, \quad t_{2}=t_{3}+\frac{t_{3} \log \left(1-\hat{y}_{1}\right)}{t_{1}}-\log \left(1-\hat{y}_{2}\right), \quad p=\hat{y}_{3} .
$$

Moreover, the Jacobian of the inverse mapping of $\mathbf{r}$ is

$$
J_{3}=\frac{1}{t_{1}\left(1-\hat{y}_{1}\right)\left(1-\hat{y}_{2}\right)} \neq 0
$$

Then, taking into account (2.16)-(2.17), the joint PDF of random vector $\left(\hat{Y}_{1}, \hat{Y}_{2}, \hat{Y}_{3}\right)=(K, Q, P)$ is given by

$f_{K, Q, P}\left(\hat{y}_{1}, \hat{y}_{2}, \hat{y}_{3}\right)$

$$
\begin{aligned}
= & f_{R R, T_{2}, P}\left(\frac{-\log \left(1-\hat{y}_{1}\right)}{t_{1}}, t_{3}+\frac{t_{3} \log \left(1-\hat{y}_{1}\right)}{t_{1}}-\log \left(1-\hat{y}_{2}\right), \hat{y}_{3}\right) \\
& \times\left|\frac{1}{t_{1}\left(1-\hat{y}_{1}\right)\left(1-\hat{y}_{2}\right)}\right| .
\end{aligned}
$$

Compounding both (8.7) and (8.8), the joint PDF of $\left(S_{n}, R_{n}, P\right)$ is determined using the PDF of random vector $\left(R R, T_{2}, P\right)$

$$
\begin{aligned}
& f_{S_{n}, R_{n}, P}(s, r, p) \\
& =f_{R R, T_{2}, P}\left(\frac{-\log \left(1-m_{1}\right)}{t_{1}}, t_{3}+\frac{t_{3} \log \left(1-m_{1}\right)}{t_{1}}-\log \left(1-m_{2}\right), p\right) \\
& \quad \times\left|\frac{1}{t_{1}\left(1-m_{1}\right)\left(1-m_{2}\right)}\right|\left|\frac{\left(\frac{s}{s_{0}}\right)^{1 / n}\left(-1+\left(\frac{s}{s_{0}}\right)^{1 / n}+p\right)}{n s\left(s-s_{0}(1-p)^{n}\right)}\right|,
\end{aligned}
$$


where $m_{1}=m_{1}\left(y_{1}, y_{2}, y_{3}\right)$ and $m_{2}=m_{2}\left(y_{1}, y_{2}, y_{3}\right)$ are the expressions introduced in (8.6) and below using the identifications $y_{1} \Rightarrow s, y_{2} \Rightarrow r$ and $y_{3} \Rightarrow p$.

Finally, considering $n$ arbitrary and marginalizing (8.9), the 1-PDFs of the subpopulation of susceptibles, $f_{1}(s, n)$, and reliants, $f_{1}(r, n)$, are obtained

$$
\begin{aligned}
& f_{1}(s, n) \\
& =\int_{\mathcal{D}\left(R_{n}, P\right)} f_{R R, T_{2}, P}\left(-\frac{\log \left(1-m_{1}\right)}{t_{1}}, t_{3}+\frac{t_{3} \log \left(1-m_{1}\right)}{t_{1}}-\log \left(1-m_{2}\right), p\right) \\
& \quad \times\left|\frac{1}{t_{1}\left(1-m_{1}\right)\left(1-m_{2}\right)}\right|\left|\frac{\left(\frac{s}{s_{0}}\right)^{1 / n}\left(-1+\left(\frac{s}{s_{0}}\right)^{1 / n}+p\right)}{n s\left(s-s_{0}(1-p)^{n}\right)}\right| \mathrm{d} p \mathrm{~d} r
\end{aligned}
$$

Now, the 1-PDF of the deceased subpopulation will be provided using that $D_{n}=1-S_{n}-R_{n}$, for each cycle $n$. To this end, Theorem 2.1 is also applied considering the following identifications

$$
\mathbf{X}=\left(S_{n}, R_{n}\right), \quad \mathbf{Y}=\left(Y_{1}, Y_{2}\right), \quad \mathbf{Y}=\mathbf{r}(\mathbf{X}),
$$

being the mapping $\mathbf{r}: \mathbb{R}^{3} \rightarrow \mathbb{R}^{3}$

$$
y_{1}=1-s-r, \quad y_{2}=r .
$$

Isolating $s$ and $r$ one gets 


$$
s=1-y_{1}-y_{2}, \quad r=y_{2}
$$

Then, taking into account (2.16)-(2.17) and that the Jacobian takes the value -1 , the joint PDF of random vector $\left(Y_{1}, Y_{2}\right)=\left(D_{n}, R_{n}\right)$ is obtained. Finally, marginalizing this latter joint PDF and considering $n$ arbitrary, it can be checked that the 1-PDF of deceased subpopulation is given by

$$
\begin{aligned}
& f_{1}(d, n) \\
& =\int_{\mathcal{D}\left(R_{n}, P\right)} f_{R R, T_{2}, P}\left(-\frac{\log \left(1-m_{1}\right)}{t_{1}}, t_{3}+\frac{t_{3} \log \left(1-m_{1}\right)}{t_{1}}-\log \left(1-m_{2}\right), p\right) \\
& \quad \times\left|\frac{1}{t_{1}\left(1-m_{1}\right)\left(1-m_{2}\right)}\right|\left|\frac{\left(\frac{1-d-r}{s_{0}}\right)^{1 / n}\left(-1+\left(\frac{1-d-r}{s_{0}}\right)^{1 / n}+p\right)}{n(1-d-r)\left((1-d-r)-s_{0}(1-p)^{n}\right)}\right| \mathrm{d} p \mathrm{~d} r .
\end{aligned}
$$

\subsubsection{Mean and variance functions. Confidence intervals}

Hereinafter, the study will be focus on susceptible subpopulation, $S_{n}$, whose $1-\mathrm{PDF}$ is given by (8.10), although the following development can be extrapolated to reliant and deceased subpopulations, using (8.11) and (8.12), respectively. The expressions for mean and variance functions are

$$
\begin{aligned}
\mu_{S_{n}} & =\mathbb{E}\left[S_{n}\right]=\int_{\mathcal{D}\left(S_{n}\right)} s f_{1}(s, n) \mathrm{d} s \\
\sigma_{S_{n}}^{2} & =\mathbb{V}\left[S_{n}\right]=\int_{\mathcal{D}\left(S_{n}\right)} s^{2} f_{1}(s, n) \mathrm{d} s-\left(\mu_{S_{n}}\right)^{2},
\end{aligned}
$$

respectively.

Furthermore, the 1-PDF is useful to construct confidence intervals. Let $\alpha \in$ $(0,1)$ and $\hat{n}$ fixed, one can determine $s_{1}=s_{1}(\hat{n})$ and $s_{2}=s_{2}(\hat{n})$ such that

$$
\int_{0}^{s_{1}} f_{1}(s, \hat{n}) \mathrm{d} s=\frac{\alpha}{2}=\int_{s_{2}}^{1} f_{1}(s, \hat{n}) \mathrm{d} s .
$$


Then, $(1-\alpha) \times 100 \%$-confidence interval is specified by

$$
1-\alpha=\mathbb{P}\left[\left\{\omega \in \Omega: S(\hat{n} ; \omega) \in\left[s_{1}, s_{2}\right]\right\}\right]=\int_{s_{1}}^{s_{2}} f_{1}(s, \hat{n}) \mathrm{d} s .
$$

In addition, it is of interest for doctors to know the probability, for example, that the proportion of susceptible subpopulation lies between $a$ and $b$ at a specific time period, say $\hat{n}$,

$$
\mathbb{P}\left[a \leq S_{\hat{n}} \leq b\right]=\int_{a}^{b} f_{1}(s, \hat{n}) \mathrm{d} s .
$$

\subsubsection{Distribution of time until a given proportion of population remains susceptible, reliant or deceased}

In practice, it is useful to know when the percentage of susceptibles, reliants and deceaseds in population will attain a certain level. This motivates the computation, in a first step, of the distribution, $N_{S}$, of time until a given proportion of population, $\rho_{S}$, remains susceptible. The same can be said for reliant and deceased subpopulations.

In order to compute the PDF of $N_{S}$ for a fixed proportion of susceptibles, $\rho_{S}$, first $n=N_{S}$ is isolated from the first component of the exact solution, given by (8.5), of IVP (8.1)-(8.2)

$$
N_{S}=\frac{\log \left(\frac{\rho_{S}}{s_{0}}\right)}{\log \left(\mathrm{e}^{-t_{1} R R}+\mathrm{e}^{-\left(T_{2}+t_{3}(R R-1)\right)}-1\right)} .
$$

Notice that the expression (8.14) depends only on RVs $R R$ and $T_{2}$. Hence, RVT technique, i.e., Theorem 2.1 is applied to

$$
\mathbf{X}=\left(R R, T_{2}\right), \quad \mathbf{Y}=\left(Y_{1}, Y_{2}\right), \quad \mathbf{Y}=\mathbf{r}(\mathbf{X}),
$$

being

$$
y_{1}=r r, \quad y_{2}=\frac{\log \left(\frac{\rho_{S}}{s_{0}}\right)}{\log \left(\mathrm{e}^{-t_{1} r r}+\mathrm{e}^{-\left(t_{2}+t_{3}(r r-1)\right)}-1\right)} .
$$


Isolating $r r$ and $t_{2}$, one gets

$$
r r=y_{1}, \quad t_{2}=t_{3}\left(1-y_{1}\right)-\log \left[\left(\frac{\rho_{S}}{s_{0}}\right)^{1 / y_{2}}+1-\mathrm{e}^{-t_{1} y_{1}}\right]
$$

The Jacobian of the inverse of mapping $\mathbf{r}$ is given by

$$
J_{3}=\frac{\left(\frac{\rho_{S}}{s_{0}}\right)^{1 / y_{2}} \log \left(\frac{\rho_{S}}{s_{0}}\right)}{y_{2}^{2}\left(1-\mathrm{e}^{-t_{1} y_{1}}+\left(\frac{\rho_{S}}{s_{0}}\right)^{1 / y_{2}}\right)} .
$$

Then, taking into account (2.16)-(2.17) the joint PDF of random vector $\left(Y_{1}, Y_{2}\right)$ $=\left(R R, N_{s}\right)$ is obtained. Finally, marginalizing with respect to RV $R R$, the expression of the PDF of $N_{S}$, for each $\rho_{S}$ fixed, is

$$
\begin{aligned}
f_{1}\left(n, \rho_{S}\right)= & \int_{\mathcal{D}(R R)} f_{R R, T_{2}}\left(r r, t_{3}(1-r r)-\log \left[\left(\frac{\rho_{S}}{s_{0}}\right)^{1 / n}+1-\mathrm{e}^{-t_{1} r r}\right]\right) \\
& \times\left|\frac{\left(\frac{\rho_{S}}{s_{0}}\right)^{1 / n} \log \left(\frac{\rho_{S}}{s_{0}}\right)}{n^{2}\left(1-\mathrm{e}^{-t_{1} r r}+\left(\frac{\rho_{S}}{s_{0}}\right)^{1 / n}\right)}\right| \mathrm{d} r r .
\end{aligned}
$$

\subsection{Probabilistic cost-effectiveness analysis}

Cost-effectiveness analysis is useful to perform an economic evaluation of sanitary interventions. Incremental cost-effectiveness ratio, $C E$, can be used in order to prioritise sanitary interventions and then maximizing benefits taking into account available budgets $[53,74] . C E$ is a ratio defined from costs and effectivenesses of two alternatives. $C E$ is defined as

$$
C E=\frac{C_{2}-C_{1}}{E_{2}-E_{1}}
$$


where $C_{i}$ and $E_{i}, i=1,2$, are the cost and the effectiveness of alternative $i$, respectively. Hereinafter, it will be assumed that $C_{i}$ and $E_{i}, i=1,2$ are RVs. In the following, the 1-PDF of $C E$ will be computed to compare two treatments for stroke disease, being the second more effective than the first, but the first cheaper. Then, differences between both are transition matrix $T$ (particularly the relative risk) and the cost. To obtain the 1-PDF of $C E$, first it shall been determined the expression of total effectiveness given by the QALY (Quality Adjusted Life Year), for each treatment. In the context of medical Markov models, QALY has already been used, see for instance [45]. QALY is the sum of effectiveness of susceptibles, reliants and deceaseds. In addition, these three effectivenesses are the sum of the effectiveness in each cycle until the value $n$ of total years considered for the study. This effectiveness is the product of number of susceptibles, reliants or deceaseds in each cycle $1 \leq j \leq n$, the utility appropriate for each state and a certain constant, which depends on a discount rate $r$. These magnitudes will be detailed later. With this aim, utility must be know, or the value of life's quality, where 0 value corresponds to death and 1 value represents that stroke disease has not been suffered by individuals, $[64,73,84]$. Then, $U_{S}=1$ and $U_{D}=0$ will be considered the utilities of susceptibles and deceaseds, respectively. For reliants, the utility, say $U_{R}$, will be modeled through a RV. Taking into account the extant literature, $r=0.03$ $(3 \%)$ is considered the discount rate $[3,6,64]$. Then, the QALY is given by

$$
E_{i}=\sum_{j=1}^{n} \frac{\mathbb{E}\left[S_{j, i}\right]}{(1+r)^{j-1}}+U_{R} \sum_{j=1}^{n} \frac{\mathbb{E}\left[R_{j, i}\right]}{(1+r)^{j-1}}, \quad i=1,2,
$$

where $\mathbb{E}\left[S_{j, i}\right]$ and $\mathbb{E}\left[R_{j, i}\right]$ are the average number of susceptibles and reliants of alternative $i \in\{1,2\}$, for each cycle $j, 1 \leq j \leq n$, respectively. The second step is to determine the expression of total cost of each treatment. The same structure that in case of QALY will be followed. On the one hand, it will be considered that cost of each treatment for susceptible subpopulation is $C S_{i}=a_{i} W$, where $a_{i}$ is the cost, in euros, of medicine per kilogram and $W$ is a RV that represents the weight of the individual to be studied. On the other hand, dependence cost will be considered a RV, denoted by $C R$. This RV is assumed to be the same in both treatments. Then, the cost in each treatment is

$$
C_{i}=C S_{i} \sum_{j=1}^{n} \frac{\mathbb{E}\left[S_{j, i}\right]}{(1+r)^{j-1}}+C R \sum_{j=1}^{n} \frac{\mathbb{E}\left[R_{j, i}\right]}{(1+r)^{j-1}}, \quad i \in\{1,2\}
$$


Substituting expressions (8.17)-(8.18) into (8.16), one gets

$$
C E=\frac{W d_{1}+C R d_{2}}{d_{3}+U_{R} d_{2}}
$$

where

$$
\begin{aligned}
d_{1} & =\sum_{j=1}^{n} \frac{a_{2} \mathbb{E}\left[S_{j, 2}\right]-a_{1} \mathbb{E}\left[S_{j, 1}\right]}{(1+r)^{j-1}}, \\
d_{2} & =\sum_{j=1}^{n} \frac{\mathbb{E}\left[R_{j, 2}\right]-\mathbb{E}\left[R_{j, 1}\right]}{(1+r)^{j-1}}, \\
d_{3} & =\sum_{j=1}^{n} \frac{\mathbb{E}\left[S_{j, 2}\right]-\mathbb{E}\left[S_{j, 1}\right]}{(1+r)^{j-1}} .
\end{aligned}
$$

Now, applying RVT technique, i.e. Theorem 2.1, the 1-PDF of $C E$ from the PDF of random vector $\left(W, C R, U_{R}\right)$, which is assumed to be known, it obtained

$$
f_{1}(c e, n)=\int_{\mathcal{D}\left(C R, U_{R}\right)} f_{W, C R, U_{R}}\left(\frac{c e\left(d_{3}+u_{r} d_{2}\right)-c r d_{2}}{d_{1}}, c r, u_{r}\right)\left|\frac{d_{3}+u_{r} d_{2}}{d_{1}}\right| \mathrm{d} u_{r} \mathrm{~d} c r .
$$

\subsection{Simulating stroke disease using real data}

In this section, it will be shown the results (simulations) for Markov model (8.1)-(8.2) in order to study stroke disease. These simulations are built using the results established in Sections 8.2 and 8.3 and considering medical information from [64].

As it is plausible from a practical standpoint, hereinafter it will be assumed that, at the beginning, the whole population is susceptible, then, initial condition is $\left(s_{0}, r_{0}, 0\right)=(1,0,0)$. Based upon [64], the following probability distributions for model inputs parameters are considered:

- Relative risk, $R R$, is a Lognormal RV with parameters (1.793; 0.143), i.e., $\log (R R) \sim \mathrm{N}(1.793 ; 0.143)$.

- Transition $R \rightarrow D$ is modelled by RV $P$, which is assumed to be a Beta distribution with parameters $(80 ; 120)$, i.e., $P \sim \operatorname{Be}(80 ; 120)$. 
- Deceased rate due to any cause, $T_{2}$, is assumed to be an Uniform distribution on interval ]0.02127, 0.02227[, $T_{2} \sim \mathrm{U}(] 0.02127,0.02227[)$.

With regard to non-mortal stroke rate, $t_{1}$, and stroke deceased rate, $t_{3}$, it is assumed that $t_{1}=0.00111$ and $t_{3}=0.00176$, respectively. These values have been taken from reference [64], taking into account that these rates correspond to a group of individuals with 65 years old. Notice that the previous theoretical results can be applied because RVs $R R$ and $T_{2}$, with the distributions specified above, satisfy condition (8.3).

In Figure 8.2, the 1-PDFs of susceptible, reliant and deceased subpopulations, given by expressions (8.10)-(8.12), have been plotted. These graphical representations have been made in periods $\{1,2, \ldots, 25\}$, assuming that RVs $R R$, $P$ and $T_{2}$ are independent. From Figure 8.2, one observes that percentage of susceptibles decreases as time increases. Besides, percentage of reliant increases at the beginning, specifically from $n=1$ to $n=6$, and afterwards this percentage tends to zero.

On the other hand, the deceased subpopulation is an absorbent state, therefore in the long-term all population will reach this state. This behaviour is in agreement with the results shown in Figure 8.2. From this graphical representation it can be observed that both percentage of dead and its variability increase over time. This same behaviour is observed to susceptible subpopulation for the periods plotted in Figure 8.2, although it will decrease as time goes on. Finally, the shape of the 1-PDF, $f_{1}(r, n)$, depicted in Figure 8.2 becomes sharp as standard deviation decreases.

In Figure 8.3, mean plus/minus standard deviation functions of the three subpopulations are shown. Notice that graphical representations exhibited in Figure 8.2 and Figure 8.3 are in agreement.

The computation of the 1-PDF is very useful in applications since from it, as seen previously in Subsection 8.2.1, one can compute exact confidence intervals in order to construct probabilistic predictions. In addition, it permits the computation of the probability associated to sets of interest. For instance, from expression (8.13) applied to reliant subpopulation, one can obtain the probability that proportion of reliants that lies between $a=0.010(1 \%)$ and $b=0.015(1.5 \%)$ in the time $\hat{n}=5$ is, approximately 0.7 ,

$$
\mathbb{P}\left[0.010 \leq R_{5} \leq 0.015\right]=\int_{0.010}^{0.015} f_{1}(r, 5) \mathrm{d} r=0.7006 .
$$



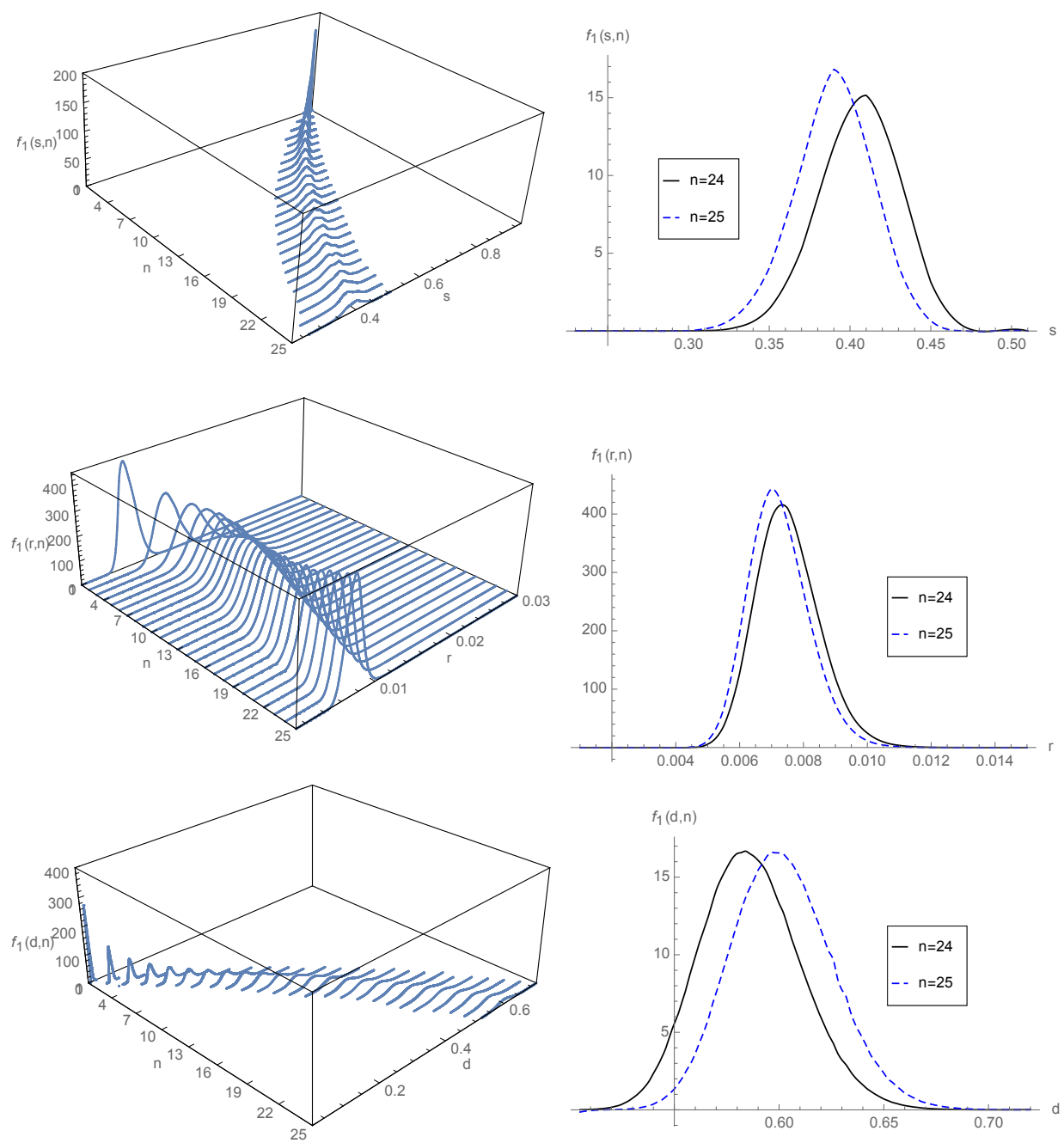

Figure 8.2: Plot of the 1-PDFs, $f_{1}(s, n), f_{1}(r, n)$ and $f_{1}(d, n)$, given by $(8.10),(8.11)$ and (8.12) respectively, at values $n \in\{1,2, \ldots, 25\}$ (left: top, center and bottom respectively). For the sake of clarity last times, $n=24$ and $n=25$, have been magnified (right). 


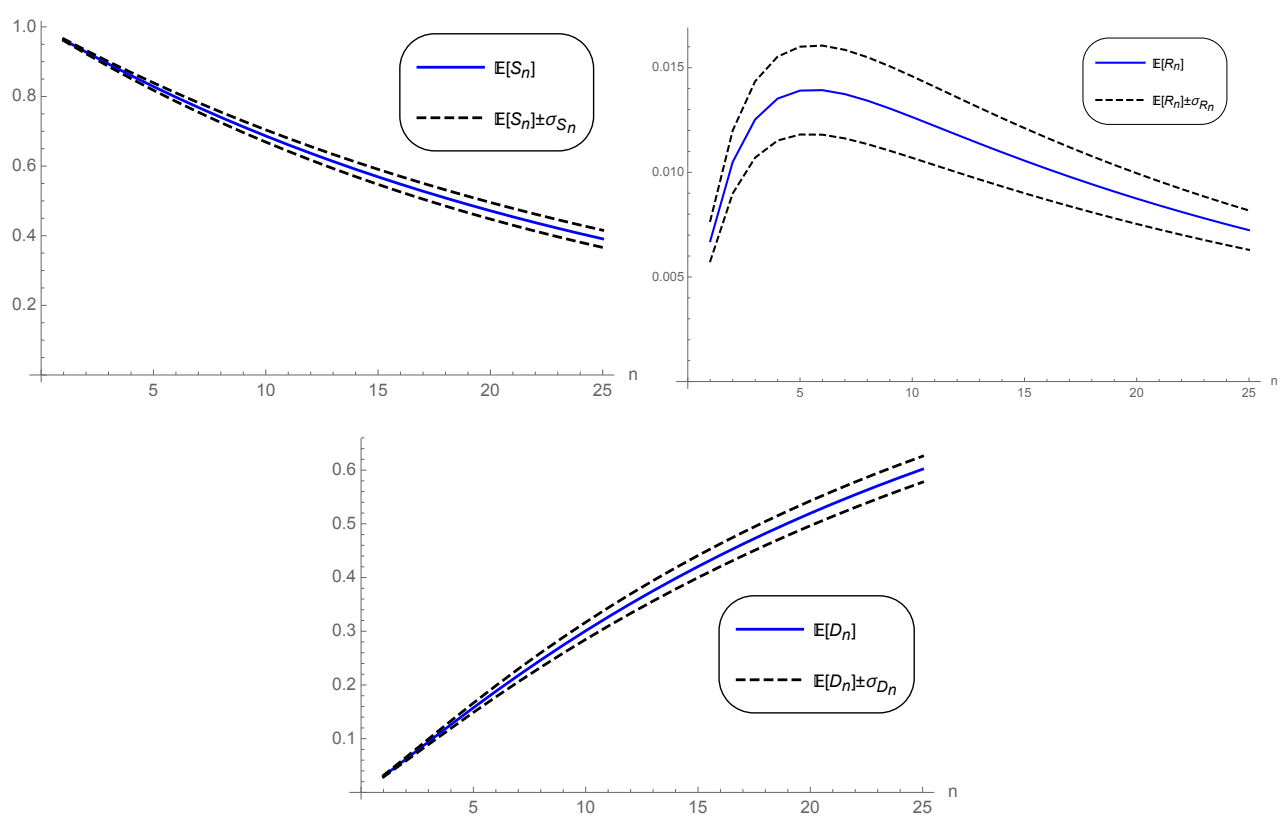

Figure 8.3: Plot of expectation plus/minus standard deviation functions of susceptible, reliant and deceased subpopulations (top left, top right and bottom, respectively).

Now, the PDFs of time until a given proportion of population remains susceptible, reliant or deceased are determined. For susceptible subpopulation this has been done using expression (8.15). Figure 8.4 shows this PDF for the following values of $\rho_{S} \in\{0.2,0.3,0.4,0.5,0.6,0.7,0.8,0.9\}$.

From the PDF of $N_{S}$, one can compute the expectation of RV $N_{S}$ for a fixed value of $\rho_{S}$, like 0.70 ,

$$
\mathbb{E}\left[N_{S}\right]=\int_{0}^{\infty} n f_{1}(n, 0.70) \mathrm{d} n=9.5190 .
$$

This means that, approximately, the middle of 10-th cycle (since the study starts at $n=0$ ) represents the average time until $70 \%$ of the population will be susceptible. This can also be seen graphically in Figure 8.4. Table 8.1 collects the expectation, $\mathbb{E}\left[N_{S}\right]$, for different values of $\rho_{S}$. This is a key information for doctors when they want to study the evolution of susceptibles of stroke disease in a group of patients. 


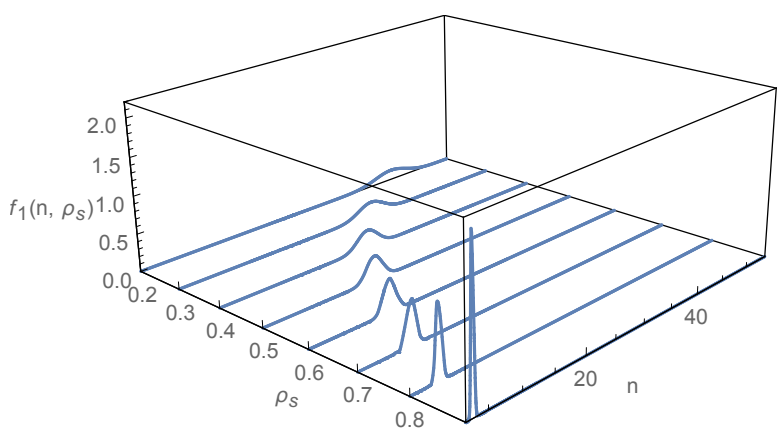

Figure 8.4: Plot of the PDF of time $N_{S}$ until a proportion $\rho_{S} \in\{0.2,0.3,0.4,0.5,0.6$, $0.7,0.8,0.9\}$ of population remains susceptible.

\begin{tabular}{ccccc}
\hline$\rho_{S}$ & 0.2 & 0.3 & 0.4 & 0.5 \\
\hline $\mathbb{E}\left[N_{S}\right]$ & 42.9520 & 32.1312 & 24.4538 & 18.4980 \\
\hline \hline$\rho_{S}$ & 0.6 & 0.7 & 0.8 & 0.9 \\
\hline $\mathbb{E}\left[N_{S}\right]$ & 13.6327 & 9.5189 & 5.9553 & 2.8118 \\
\hline
\end{tabular}

Table 8.1: Expectation of time $N_{S}$ until a proportion, $\rho_{S}$, of population remains susceptible for different values $\rho_{S}$.

In order to obtain the PDFs of RVs $N_{R}$ and $N_{D}$, that denote the time until a proportion of population, $\rho_{R}$ and $\rho_{D}$, remains reliant or deceased, respectively, numerical methods have been applied. This decision has been made because $n$ cannot be isolated from the second and third components of solution given by (8.5). To illustrate the process, below the steps for reliant subpopulation where $\rho_{R}$ is assumed to be fixed are specified:

- Step 1: To sample 500000 values, say $\left(r r, t_{2}, p\right)$, according to the specific distributions assumed for RVs $R R, T_{2}$ and $P$.

- Step 2: For each sampled value $\left(r r, t_{2}, p\right)$, to apply Newton method to calculate the value $n$ of $N_{R}$ solving the nonlinear equation defined by the second component of (8.5), that corresponds to reliant subpopulation, substituting $R R \Rightarrow r r, T_{2} \Rightarrow t_{2}$ and $P \Rightarrow p$. This process provides 500000 values for $n$ of $N_{R}$. 
- Step 3: To plot the histogram of 500000 values of $n$. A normalization of this histogram is an approximation of the PDF of $N_{R}$.

Since numerical convergence of Newton method heavily depends on the seed or starting point, say $s p$, for example, in the case that $\rho_{R}=0.006$, two graphical representations for the PDF of $N_{R}$ are shown in Figure 8.5. Specifically, taking values $s p=1$ and $s p=25$, the PDF of the time $N_{R}$ has been obtained for cycles $n=1$ and $n=30$, respectively. This is due because proportion of reliants reaches value $\rho_{R}=0.006$ in those two cycles. Whereas for the case $\rho_{R}=0.0183$, Newton method always converges for cycle $n=5$, thus defining a single PDF for $N_{R}$. This PDF has been plotted in Figure 8.6 taking as seed value $s p=2$.
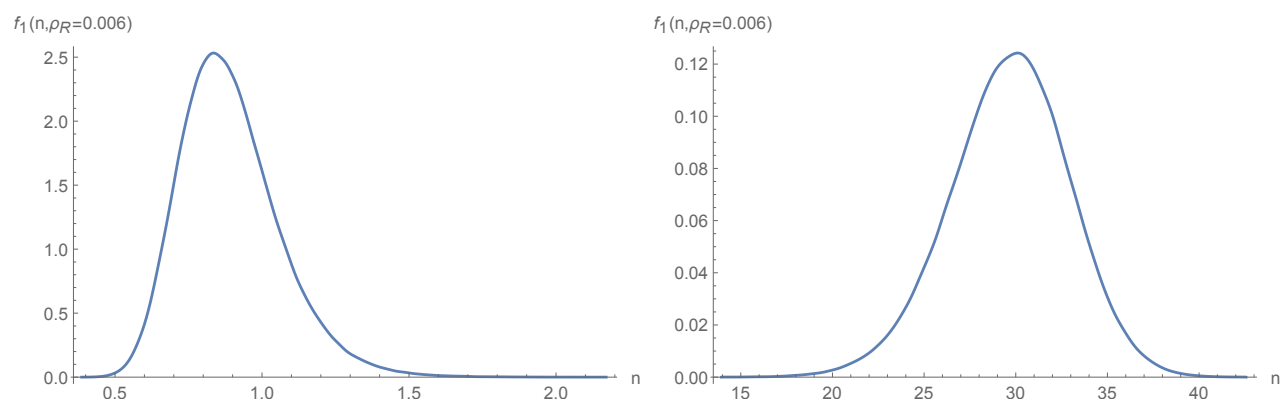

Figure 8.5: Plot of the PDF of the time $N_{R}$ until a proportion $\rho_{R}=0.006$ of the population remains reliant, using as seed points $s p=1$ (left) and $s p=25$ (right).

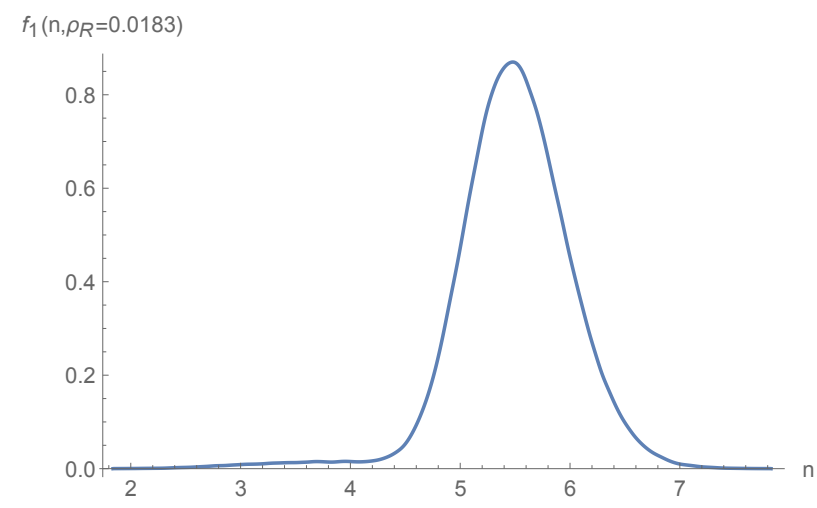

Figure 8.6: Plot of the PDF of time $N_{R}$ until a proportion $\rho_{R}=0.0183$ of population remains reliant, using as seed point $s p=2$. 
Regarding deceased subpopulation, the same steps described previously have been followed. In Figure 8.7, the PDF of $N_{D}$ has been plotted for the following values of percentage $\rho_{D} \in\{0.1,0.2,0.3,0.4,0.5,0.6,0.7,0.8,0.9\}$, where $s p=3$ has been taken as seed point, for each value of $\rho_{D}$. In this case, to every value of $\rho_{D}$ corresponds a unique value of $n$.
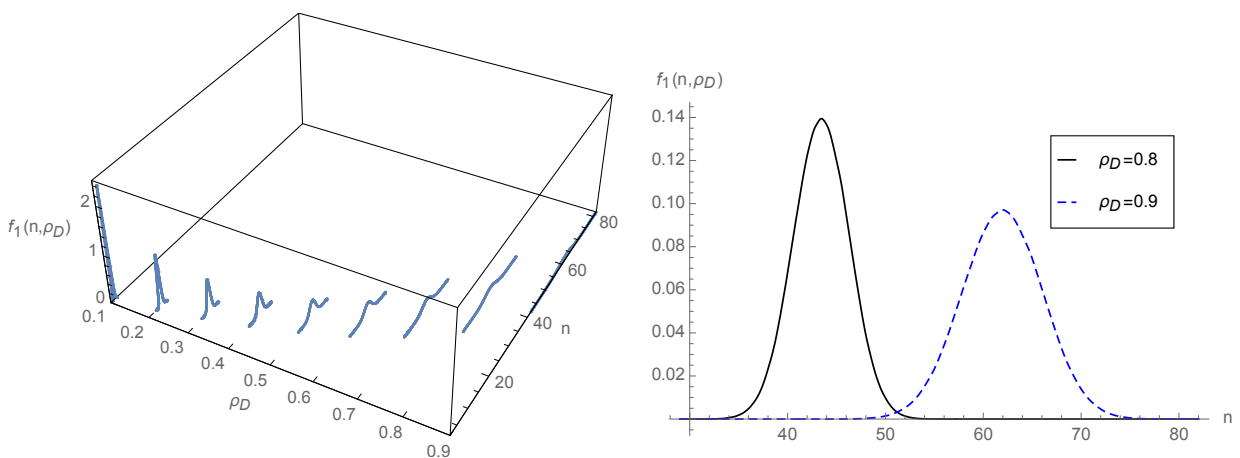

Figure 8.7: Plot of the PDF of time $N_{D}$ until a proportion $\rho_{D} \in\{0.1,0.2,0.3,0.4,0.5,0.6$, $0.7,0.8,0.9\}$ of population remains dead (left). For the sake of clarity last proportions, $\rho_{D}=0.8$ and $\rho_{D}=0.9$, have been magnified (right).

Finally, the 1-PDF of $C E$ given by (8.19) has been computed. From it, mean and confidence intervals will also be computed. This will be done for different ages, 30, 65 and 71. This decision has been made because involved rates for each age are different. Computations have been carried out taking the following probability distributions for random inputs:

- Relative risk for the first alternative (the less efficient), $R R_{1}$, is a Lognormal RV with parameters $(1.793 ; 0.143)$, i.e., $\log \left(R R_{1}\right) \sim \mathrm{N}(1.793 ; 0.143)$.

- Relative risk for the second alternative (the most efficient), $R R_{2}=R R_{1} B$, where $B$ is the benefit.

- $B$ is a Lognormal RV with parameters $(-0.964 ; 0.163)$, i.e., $\log (B) \sim$ $\mathrm{N}(-0.964 ; 0.163)$.

- Transition $R \rightarrow D$ is modelled by RV $P$, which is assumed to be a Beta distribution with parameters $(80 ; 120)$, i.e., $P \sim \operatorname{Be}(80 ; 120)$.

- Deceased rate due to any cause, $T_{2}$, is assumed to be a RV with a uniform distribution on the interval ] $t_{2}-0.0001, t_{2}+0.0001\left[\right.$, i.e., $T_{2} \sim \mathrm{U}(] t_{2}-$ $0.0001, t_{2}+0.0001[)$, where $t_{2}$ is a fixed value, which depends on age, and that will be specified below. 
- Dependence cost, $C R$, is a Lognormal RV with parameters $(6.936 ; 0.643)$, i.e., $\log (C R) \sim \mathrm{N}(6.936 ; 0.643)$.

- Weight, $W$, is a Gaussian RV with parameters $(75.900 ; 12.290)$, i.e., $W \sim$ $\mathrm{N}(75.900 ; 12.290)$. The prices of both treatments are $a_{1}=6.5 € / \mathrm{kg}$ and $a_{2}=65 € / \mathrm{kg}$, [64].

- Utility, $U_{R}$, is a Gaussian RV with parameters $(0.701 ; 0.347)$ i.e., $U_{R} \sim$ $\mathrm{N}(0.701 ; 0.347)$.

Above, $t_{1}, t_{2}$ and $t_{3}$ are rates, which depend on age of population under study. In Figure 8.8, the expectation of susceptibles, reliants and deceaseds for the two alternatives in the three ages are shown. For each one of them, it have been considered the following rates, $t_{1}, t_{2}$ and $t_{3}$, which are based on [64], and end cycles values, $n_{\max }$,

- For age of 30 years: $t_{1}=0.0000298, t_{2}=0.00169, t_{3}=0.00004$ and $n_{\max }=69$.

- For age of 65 years: $t_{1}=0.0011135, t_{2}=0.02177, t_{3}=0.00176$ and $n_{\max }=34$.

- For age of 71 years: $t_{1}=0.0031780, t_{2}=0.03616$ and $t_{3}=0.00373$ and $n_{\max }=28$.

Notice that this study is until 99 years, but another age limit can be chosen. From Figure 8.8, one observes, in all the ages, that mean of susceptibles with the second treatment is greater than considering the first, and the reverse for expectation of reliants and deceaseds. This is consistent with the fact that second alternative is better than the first.

In Figure 8.9 the 1-PDFs of $C E$, given by (8.19), have been plotted for each age from cycle 1 to 34. Notice that graphical representations shown in Figure 8.9 are in agreement with Figure 8.10, where the expectation plus/minus standard deviation functions of $C E$ for each age have been plotted. To facilitate comparison between both alternatives, the value $30000 € /$ QALY (red straight line) has also been plotted as a threshold. This benchmark value has been chosen because, according to [78], is a standard value in the literature. From Figure 8.10, one can observe that for people aged 71 years old second alternative (the most expensive), is more effective than first alternative. Naturally, for people aged 30 years old, the best alternative is the first one because they have 

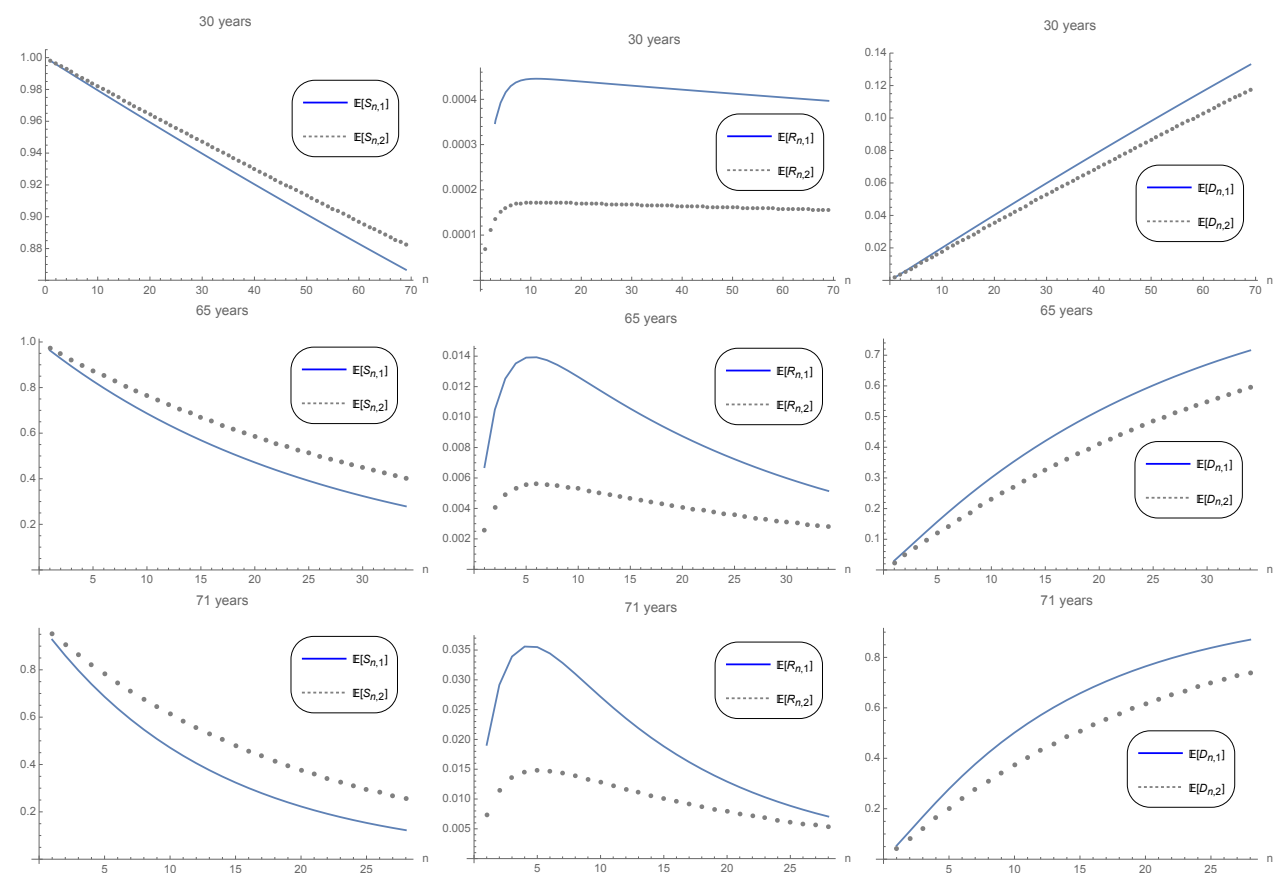

Figure 8.8: Plots of expectation of susceptibles, reliants and deceaseds for the two alternatives 1 (the cheapest) and 2 (the most expensive) in three ages: 30 years (first row), 65 years (second row) and 71 years (third row).

longer lifetime. For people aged 65 years old, it might be controversy because their $C E$ is very close to the threshold.

\subsection{Conclusions}

Although Markov models have been used extensively for modelling the dynamic of numerous diseases, few attempts have been made regarding stroke disease. Markovian approach is useful to perform clinical control of patients that suffer this disease. Indeed, Markov models allow to forecast not only the number of patients belonging to each subpopulation (susceptibles, reliants and deceaseds) at every cycle but also to account for significant medical information. In this regard, time until a given proportion of patients remain susceptible, reliant or deceased are, for example, important information in medical treatment of stroke. This is a key information to answer crucial questions like 'what is 

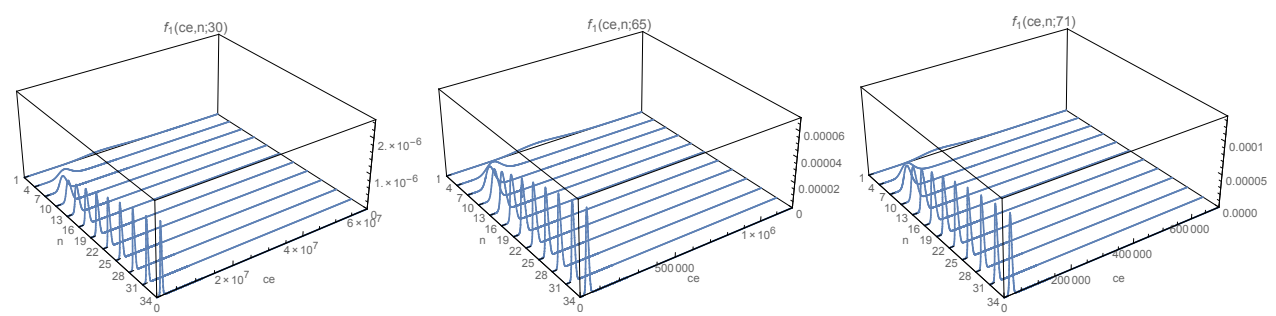

Figure 8.9: PDFs $C E$ given by (8.19) considering both alternatives in the three ages: 30 years (left), 65 years (center) and 71 years (right).
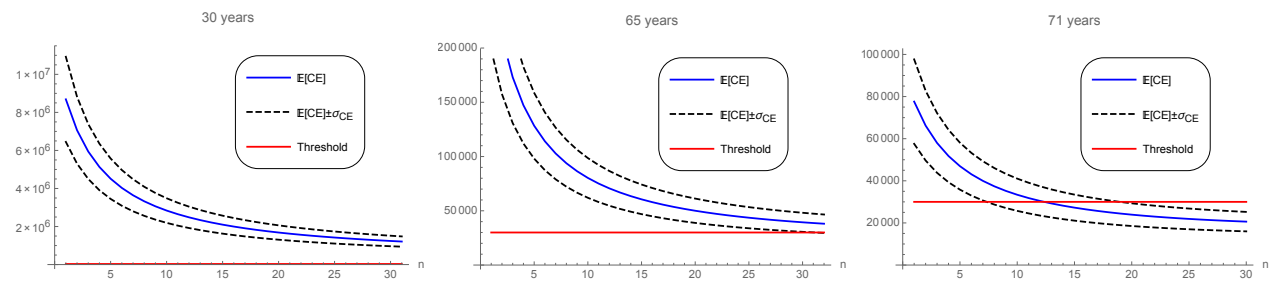

Figure 8.10: Plots of expectation plus/minus standard deviation functions of $C E$ for each age considering both alternatives in the three ages: 30 years (left), 65 years (center) and 71 years (right). Red straight line represents the threshold value $30000 € /$ QALY usually taking as reference [78].

the expected time before twenty percent, for instance, of population remains susceptible?'.

In this chapter, it has been given a technical generalization of classical Markov methodology that enables the exact determination of crucial medical information previously indicated. This generalization is aimed to improve the modelling of stroke disease when dealing with real data, although an important issue is that this technique can easily be adapted to another diseases using the markovian paradigm. The approach resorts in the so-called Random Variable Transformation method. Randomization has been done through some of entries of transition matrix of a classical Markov chain which has been previously proposed to model stroke disease. This approach allows the computation of the first probability density function of solution stochastic process, and then obtaining punctual and probabilistic predictions as well as important probabilistic information that has been underlined previously.

Moreover, a probabilistic cost-effectiveness analysis, based on the application of Random Variable Transformation technique, has been conducted. The main 
advantage of this computational approach is that results can be obtained in an exact manner rather than using simulations. 



\section{Chapter 9}

\section{Random second-order linear differential equations}

This chapter deals with the approximate computation of the first probability density function of solution stochastic process to second-order linear differential equations with random analytic coefficients about regular points under very general hypotheses. The approach is based on considering approximations of solution stochastic process via truncated power series solution obtained from random Fröbenius method together with the so-called Random Variable Transformation technique. Validity of proposed method is shown through several illustrative examples.

\subsection{Introduction}

The aim of this chapter is to provide a full probabilistic description, through the approximation of the 1-PDF, $f_{1}(x, t)$, of solution SP, $X(t)$, to the secondorder random linear differential equation

$$
\ddot{X}(t)+p(t ; A) \dot{X}(t)+q(t ; A) X(t)=0, \quad t>t_{0} \in \mathbb{R},
$$


with initial conditions

$$
X\left(t_{0}\right)=Y_{0}, \quad \dot{X}\left(t_{0}\right)=Y_{1} .
$$

In IVP (9.1)-(9.2), $A, Y_{0}$ and $Y_{1}$ are assumed to be absolutely continuous real RVs defined on a common complete probability space $(\Omega, \mathcal{A}, \mathbb{P})$. For the sake of clarity, realizations for any $\mathrm{RV}$, say $Z: \Omega \longrightarrow \mathcal{D}(Z) \subset \mathbb{R}$, will be denoted by $z(\omega) \in \mathcal{D}(Z), \omega \in \Omega$. As usual, from now $\mathcal{D}(Z)$ will be referred to as the range or codomain of $Z$. To provide more generality in the study, it will be assumed that these RVs are statistically dependent, being $f_{A, Y_{0}, Y_{1}}\left(a, y_{0}, y_{1}\right)$ the joint PDF of random vector $\left(A, Y_{0}, Y_{1}\right)$. The domain of this function will be denoted by $\mathcal{D}\left(A, Y_{0}, Y_{1}\right) \subset \mathbb{R}^{3}$. For convenience, the following notation will be introduced, that will be used later, $\mathcal{D}(A) \subset \mathbb{R}, \mathcal{D}\left(Y_{1}\right) \subset \mathbb{R}$ and $\mathcal{D}\left(A, Y_{1}\right) \subset$ $\mathbb{R}^{2}$, that stand for codomains of RVs $A$ and $Y_{1}$, and random vector $\left(A, Y_{1}\right)$, respectively. Throughout this chapter, it will be assumed that

H1 :

$$
f_{A, Y_{0}, Y_{1}}\left(a, y_{0}, y_{1}\right) \text { is continuous in } y_{0} \text { and uniformly bounded, i.e., }
$$

$$
\exists M_{f}>0:\left|f_{A, Y_{0}, Y_{1}}\left(a, y_{0}, y_{1}\right)\right|<M_{f}, \forall\left(a_{0}, y_{0}, y_{1}\right) \in \mathcal{D}\left(A, Y_{0}, Y_{1}\right) .
$$

It is remarkable that a wide range of probabilistic distributions satisfy hypothesis H1, for example, Gaussian, Uniform, Beta, Gamma, Lognormal, t-Student, etc. In particular, this condition is met for any multimodal distribution.

Henceforth, coefficients $p(t ; A)$ and $q(t ; A)$ will be assumed SPs, which depend on RV $A$, such that they are analytic about $\left(t_{0} ; a_{0}(\omega)\right)$ for every $a_{0}(\omega) \in \mathcal{D}(A)$, $\omega \in \Omega$, i.e.,

H2 :

there exists a neighbourhood $\mathcal{N}_{p, q}\left(t_{0} ; a_{0}(\omega)\right) \subset\left[t_{0},+\infty[\times \mathcal{D}(A)\right.$ where

$$
p(t ; A), q(t ; A) \text { are analytic } \forall a_{0}(\omega) \in \mathcal{D}(A), \omega \in \Omega .
$$

With the standard identification $p(t ; A) \equiv p(t ; a(\omega)), a(\omega) \in \mathcal{D}(A), \forall \omega \in \Omega$, the SP $p(t ; A)$ is analytic about $\left(t_{0}, a_{0}(\omega)\right), a_{0}(\omega) \in \mathcal{D}(A), \forall \omega \in \Omega$, if the deterministic function $p(t ; a(\omega))$ is analytic about $\left(t_{0}, a_{0}(\omega)\right)$. The same can be said for the SP $q(t ; A)$. To facilitate the subsequent analysis, hereinafter it will be assumed that $p(t ; A)$ and $q(t ; A)$ satisfy sufficient conditions in order to guarantee that IVP (9.1)-(9.2) has a unique solution. 
Notice that in order to simplify notation, $p(t ; A)$ and $q(t ; A)$ are assumed to be analytic in a common neighbourhood, $\mathcal{N}_{p, q}\left(t_{0} ; a_{0}(\omega)\right)$, which in practice will be defined by the intersection of corresponding domains of analyticity of $p(t ; A)$ and $q(t ; A)$.

Problems as (9.1)-(9.2) are important since many differential equations appearing in Mathematical Physics fall into this class. In this regard, it is worth pointing out that some recent contributions dealing with particular random differential equations of the form (9.1)-(9.2) can be found in [13, 31, 43], for example. In $[13,31]$ authors give solutions of Hermite and Airy random differential equations, respectively, using the so-called mean square and mean fourth calculus [85, 92]. In both contributions, approximations for mean and variance of solution SP are given. In [43], these two statistical moments are computed for a wide range of second-order random linear differential equations taking advantage of homotopy analysis method. Recently, in [55] an analogous study has been conducted by applying random differential transformation method developed in [91].

In this chapter, RVT technique will be used to compute approximations of $f_{1}(x, t)$. The aim of this chapter is computing approximations of the 1-PDF, $f_{1}(x, t)$, of solution SP to IVP (9.1)-(9.2), when solution is constructed by power series using the so-called Fröbenius method [88, ch.4].

The chapter is organized as follows. Some auxiliary results that will be required later will be established in Section 9.2, In Section 9.3, an approximation of the first probability density function of solution SP to IVP (9.1)-(9.2) is constructed. This approximation is based on truncated random power series of solution SP obtained via Fröbenius method and the application of RVT technique. In Section 9.3 it will also be studied the convergence of the 1-PDF of the truncated solution to the 1-PDF of the exact solution. Section 9.4 is addressed to show several illustrative examples where proposed technique is successfully applied. Finally, in Section 9.5 the main conclusions are drawn.

\subsection{Auxiliary results}

An important result that will be required later is Poincaré's expansion theorem. For the sake of completeness, its statement [90, Theorem 9.2, p. 119], [38, Theorem 5.5, p.296] is reminded. This result permits to represent the solution of a differential equation whose formulation depends on a parameter, say $\lambda$, by means of a power series of $\lambda$ parameter itself. 
Theorem 9.1 Let us consider the initial value problem

$$
\left.\begin{array}{rl}
\dot{\mathbf{W}}(t) & =\mathbf{f}(t, \mathbf{W}(t) ; \lambda), \quad t>t_{0}, \\
\mathbf{W}\left(t_{0}\right) & =\mathbf{W}_{0},
\end{array}\right\}
$$

where $\mathbf{f}:] t_{0},+\infty\left[\times \mathbb{R}^{n} \times \mathbb{R} \longrightarrow \mathbb{R}^{n}, \mathbf{f}=\left(f_{1}, \ldots, f_{n}\right)^{\top}, \mathbf{W}(t) \equiv \mathbf{W}=\left(W_{1}, \ldots, W_{n}\right)^{\top}\right.$ and $\mathbf{W}_{0}=\left(W_{0,1}, \ldots, W_{0, n}\right)^{\top}$. If $\mathbf{f}(t, \mathbf{W} ; \lambda)$ admits a convergent power series expansion about $\left(t_{0}, \mathbf{W}_{0} ; \lambda_{0}\right)$, i.e.,

$$
\begin{aligned}
& f_{i}(t, \mathbf{W} ; \lambda) \\
& =\sum_{j \geq 0} \sum_{k_{1}, \ldots, k_{n} \geq 0} \sum_{l \geq 0} c_{j, k_{1}, \ldots, k_{n}, l}^{i}\left(t-t_{0}\right)^{j}\left(W_{1}-W_{0,1}\right)^{k_{1}} \cdots\left(W_{n}-W_{0, n}\right)^{k_{n}}\left(\lambda-\lambda_{0}\right)^{l},
\end{aligned}
$$

$\lambda_{0} \in \mathbb{R}, 1 \leq i \leq n$. Then, for every $\left(t_{0}, \mathbf{W}_{0}\right)$, solution of IVP (9.5) can also be represented as the following convergent power series

$$
\mathbf{W}(t)=\sum_{l \geq 0} \mathbf{L}_{l}(t)\left(\lambda-\lambda_{0}\right)^{l} .
$$

Coefficients $\mathbf{L}_{l}(t)$ are solutions of certain coupled unhomogeneous linear system of linear differential equations.

Remark 9.1 Notice that Theorem 9.1 can be applied to IVP (9.1)-(9.2) by considering the standard change of variable

$$
\mathbf{W}(t)=\left(W_{1}(t), W_{2}(t)\right)^{\top}=(X(t), \dot{X}(t))^{\top}, \quad \mathbf{W}_{0}=\left(Y_{0} Y_{1}\right)^{\top}
$$

$$
\begin{aligned}
& \lambda=a(\omega), \omega \in \Omega \text { and } \mathbf{f}=\left(f_{1}(t, \mathbf{W}(t) ; A(\omega)), f_{2}(t, \mathbf{W}(t) ; a(\omega))\right)^{\top} \text {, being } \\
& \left.\begin{array}{l}
f_{1}(t, \mathbf{W}(t) ; a(\omega))=W_{2}(t), \\
f_{2}(t, \mathbf{W}(t) ; a(\omega))=-p(t ; a(\omega)) W_{2}(t)-q(t ; a(\omega)) W_{1}(t)
\end{array}\right\} \text { for each } \omega \in \Omega .
\end{aligned}
$$

This section will be finished stating standard notation and some classical results related to uniform convergence that will be required later. Let $E \subset \mathbb{R}^{n}$ 
be a Lebesgue measurable set with finite measure. In the following, the pair $\left(\mathcal{L}^{1}(E),\|\cdot\|_{1}\right)$ will denote the normed space of mappings $f: E \subset \mathbb{R}^{n} \longrightarrow \mathbb{R}$ such that they are absolutely Lebesgue integrable, i.e.,

$$
\|f\|_{1}=\int_{E}|f| \text { exists and is finite. }
$$

Proposition 9.1 Let $E \subset \mathbb{R}^{n}$ be a Lebesgue measurable set with finite measure and let $\left\{f_{N}(e): N \geq 0\right\} \subset \mathcal{L}^{1}(E)$ such that $f_{N}(e)$ converges uniformly in $E$, i.e., $f_{N}(e) \underset{N \rightarrow+\infty}{\stackrel{\text { uniformly } E}{\longrightarrow}} f(e)$. Then, $f(e) \in \mathcal{L}^{1}(E)$ and

$$
\lim _{N \rightarrow+\infty} \int_{E} f_{N}(e) \mathrm{d} e=\int_{E} f(e) \mathrm{d} e .
$$

Proposition 9.2 Let $\left\{g_{N}\left(z_{1}, z_{2}\right): N \geq 0\right\}$ and $\left\{h_{N}\left(z_{1}, z_{2}\right): N \geq 0\right\}$ be two sequences of functions such that $h_{N}\left(z_{1}, z_{2}\right) \neq 0, \forall N \geq 0, \forall\left(z_{1}, z_{2}\right) \in D \subset \mathbb{R}^{2}$. Let us assume that both converge uniformly in $D$, i.e.,

$$
g_{N}\left(z_{1}, z_{2}\right) \underset{N \rightarrow+\infty}{\stackrel{\text { uniformly } D}{\longrightarrow}} g\left(z_{1}, z_{2}\right), \quad h_{N}\left(z_{1}, z_{2}\right) \underset{N \rightarrow+\infty}{\stackrel{\text { uniformly } D}{\longrightarrow}} h\left(z_{1}, z_{2}\right)
$$

being $h\left(z_{1}, z_{2}\right) \neq 0, \forall\left(z_{1}, z_{2}\right) \in D$, and there exist positive constants $M_{g}, m_{h}$ and $M_{h}$ such that

$$
\left|g_{N}\left(z_{1}, z_{2}\right)\right|<M_{g}, \quad 0<m_{h}<\left|h_{N}\left(z_{1}, z_{2}\right)\right|<M_{h}, \quad \forall N \geq 0, \forall\left(z_{1}, z_{2}\right) \in D
$$

Then

$$
\underset{h_{N}\left(z_{1}, z_{2}\right)}{\stackrel{\text { uniformly } D}{\longrightarrow}} \underset{N \rightarrow+\infty}{\frac{g\left(z_{1}, z_{2}\right)}{h\left(z_{1}, z_{2}\right)}} .
$$

Proposition 9.3 Let $D_{u, v}$ be a subset of $\mathbb{R}^{2}$ such that

$$
\sup \left\{|v|:(u, v) \in D_{u, v}, \forall u\right\}<+\infty
$$

and let $\left\{l_{N}(u): N \geq 0\right\}$ be a sequence of functions uniformly convergent in $D_{u}=\left\{u:(u, v) \in D_{u, v}\right\}$, i.e., 


$$
l_{N}(u) \underset{N \rightarrow+\infty}{\stackrel{\text { uniformly } D_{u}}{\longrightarrow}} l(u) .
$$

Then, the sequence of functions $b_{N}(u, v)=c+v l_{N}(u)$, where $c$ is a constant, converges uniformly in $D_{u, v}$, i.e.,

$$
b_{N}(u, v) \underset{\text { uniformly } D_{u, v}}{\stackrel{N \rightarrow+\infty}{\longrightarrow}} b(u, v)
$$

being $b(u, v)=c+v l(u)$.

Proposition 9.4 Let $\left\{\gamma_{N}\left(z_{1}, z_{2}\right): N \geq 0\right\}$ be a sequence of functions such that converges uniformly in $D \subset \mathbb{R}^{2}$, i.e.,

$$
\gamma_{N}\left(z_{1}, z_{2}\right) \underset{N \rightarrow+\infty}{\stackrel{\text { uniformly } D}{\longrightarrow}} \gamma\left(z_{1}, z_{2}\right)
$$

Let $\phi: D_{\phi} \subset \mathbb{R}^{3} \longrightarrow \mathbb{R}$ be a mapping such that is continuous with respect to its second variable. Then, the sequence of functions defined by $\psi_{N}\left(z_{1}, z_{2}\right)=$ $\phi\left(z_{1}, \gamma_{N}\left(z_{1}, z_{2}\right), z_{2}\right)$ converges uniformly in $D$, i.e.,

$$
\psi_{N}\left(z_{1}, z_{2}\right) \underset{N \rightarrow+\infty}{\stackrel{\text { uniformly } D}{\longrightarrow}} \psi\left(z_{1}, z_{2}\right)
$$

where $\psi\left(z_{1}, z_{2}\right)=\phi\left(z_{1}, \gamma\left(z_{1}, z_{2}\right), z_{2}\right)$.

\subsection{Computing the approximation of the first probability density function}

In agreement with Fröbenius method [88, Chapter 4], it is well-known that solution of IVP (9.1)-(9.2) can be represented in the form

$$
X(t)=Y_{0} S_{1}(t ; A)+Y_{1} S_{2}(t ; A)
$$

being

$$
S_{1}(t ; A)=\sum_{n \geq 0} C_{n}(A)\left(t-t_{0}\right)^{n}, \quad S_{2}(t ; A)=\sum_{n \geq 0} D_{n}(A)\left(t-t_{0}\right)^{n} .
$$


Coefficients $C_{n}(A)$ and $D_{n}(A)$ are recursively determined from initial conditions $Y_{0}$ and $Y_{1}$ and a second-order linear difference equation. It is worth pointing out that $S_{1}(t ; A)$ and $S_{2}(t ; A)$ have no common powers $\left(t-t_{0}\right)^{n}$, hence indexes $n \geq 0$ that appear in the two series defining $S_{1}(t ; A)$ and $S_{2}(t ; A)$ in (9.9) really lie in disjoint subsets of non-negative integers $\mathbb{N}=\{0,1,2, \ldots\}$. This issue has been omitted in (9.9) just to simplify notation. Also notice that, from the initial condition $X\left(t_{0}\right)=Y_{0}$ (see (9.2)) and (9.8)-(9.9), one can assure that $C_{0}(A)=1$ and $D_{0}(A)=0$.

Remark 9.2 On the one hand, interpreting $R V A$ as a parameter indexed by $\omega \in \Omega, A=\{a(\omega): \omega \in \Omega\}$, and under hypothesis H2 given in (9.4), Theorem 9.1 guarantees that solution $X(t)$ can also be represented as a power series of parameter $a(\omega), \omega \in \Omega$. Therefore, $S_{1}(t ; A)$ and $S_{2}(t ; A)$ do. On the other hand, taking into account uniqueness of solution of IVP (9.1)-(9.2), both series expansions (as powers of $t-t_{0}$ and as powers of $a(\omega)-a_{0}(\omega)$ ) match. Thus, the two series $S_{1}(t ; A)$ and $S_{2}(t ; A)$, given by $(9.9)$, converge in certain domains $\mathcal{N}_{S_{1}}\left(t_{0} ; a_{0}(\omega)\right)$ and $\mathcal{N}_{S_{2}}\left(t_{0} ; a_{0}(\omega)\right)$, respectively, for all $a_{0}(\omega) \in$ $\mathcal{D}(A), \omega \in \Omega$. In addition, uniform convergence takes place in every closed set contained in $\mathcal{N}_{S_{1}}\left(t_{0} ; a_{0}(\omega)\right)$ and $\mathcal{N}_{S_{2}}\left(t_{0} ; a_{0}(\omega)\right)$. Notice that the domain of convergence, $\mathcal{N}_{X}\left(t_{0} ; a_{0}(\omega)\right)$, of power series solution $X(t)$ given in (9.8), satisfies $\mathcal{N}_{p, q}\left(t_{0} ; a_{0}(\omega)\right) \subseteq \mathcal{N}_{X}\left(t_{0} ; a_{0}(\omega)\right)=\mathcal{N}_{S_{1}}\left(t_{0} ; a_{0}(\omega)\right) \cap \mathcal{N}_{S_{2}}\left(t_{0} ; a_{0}(\omega)\right)$ for all $a_{0}(\omega) \in \mathcal{D}(A), \omega \in \Omega$, where $\mathcal{N}_{p, q}\left(t_{0} ; a_{0}(\omega)\right)$ is defined in hypothesis H2 (see (9.4)).

Approximation of the 1-PDF, $f_{1}(x, t)$, to the SP $X(t)$ given by $(9.8)$ will be computed from truncation, say $X_{N}(t)$, of $X(t)$,

$$
X_{N}(t)=Y_{0} S_{1}^{N}(t ; A)+Y_{1} S_{2}^{N}(t ; A)
$$

where

$$
S_{1}^{N}(t ; A)=\sum_{n=0}^{N} C_{n}(A)\left(t-t_{0}\right)^{n}, \quad S_{2}^{N}(t ; A)=\sum_{n=0}^{N} D_{n}(A)\left(t-t_{0}\right)^{n}
$$

being $N$ a non-negative integer previously fixed. Truncation is required to keep burden computation feasible in dealing with approximation of the 1-PDF. 
Remark 9.3 On the one hand, it has been previously shown that $C_{0}(A)=1$, hence from (9.11) one gets

$$
S_{1}^{N}\left(t_{0} ; A\right)=C_{0}(A)=1 \neq 0, \quad \forall N \geq 0 .
$$

From Remark 9.2, it is know that $S_{1}(t ; A)$ can be represented through a power series in $t-t_{0}$ and $a(\omega)-a_{0}(\omega)$ for every $a_{0}(\omega), \omega \in \Omega$. Hence, $S_{1}^{N}(t ; A)$ is continuous at $\left(t_{0}, a_{0}(\omega)\right)$ for each $a_{0}(\omega), \omega \in \Omega$, since it is a polynomial in both variables $(t, a(\omega))$. This guarantees the existence of a neighbourhood, that without loss of generality can be taken as one introduced in Remark 9.2, $\mathcal{N}_{X}\left(t_{0} ; a_{0}(\omega)\right)$, and a positive constant, $m_{S_{1}}$, such that

$$
\begin{aligned}
& 0<m_{S_{1}}<\left|S_{1}^{N}(t ; a(\omega))\right|, \\
& \forall(t, a(\omega)) \in \mathcal{N}_{X}\left(t_{0} ; a_{0}(\omega)\right), \omega \in \Omega, \forall a_{0}(\omega) \in \mathcal{D}(A), \forall N \geq 0 .
\end{aligned}
$$

On the other hand, in agreement with Remark 9.2 it is know that $S_{i}(t ; a)$, $i=1,2$, are analytic in a common neighbourhood $\mathcal{N}_{X}\left(t_{0} ; a_{0}(\omega)\right)$ and that both series $S_{i}(t ; a), i=1,2$, converge uniformly in every closed set $\mathcal{N}_{X}^{*}\left(t_{0} ; a_{0}(\omega)\right)$ contained in $\mathcal{N}_{X}\left(t_{0} ; a_{0}(\omega)\right)$ for all $a_{0}(\omega) \in \mathcal{D}(A), \omega \in \Omega$. This guarantees the existence of a positive constant $M_{S}$, such that

$$
\begin{aligned}
& \left|S_{i}^{N}(t ; a(\omega))\right|<M_{S}, \\
& \forall(t, a(\omega)) \in \mathcal{N}_{X}^{*}\left(t_{0} ; a_{0}(\omega)\right) \subset \mathcal{N}_{X}\left(t_{0} ; a_{0}(\omega)\right), \omega \in \Omega, \\
& \forall a_{0}(\omega) \in \mathcal{D}(A), \forall N \geq 0, i=1,2 .
\end{aligned}
$$

Summarizing, from (9.13) and (9.14) the following bound for $S_{1}(t ; A)$ is obtained

$$
\begin{aligned}
& 0<m_{S_{1}}<\left|S_{1}^{N}(t ; a(\omega))\right|<M_{S}, \\
& \forall(t, a(\omega)) \in \mathcal{N}_{X}^{*}\left(t_{0} ; a_{0}(\omega)\right), \omega \in \Omega, \forall a_{0}(\omega) \in \mathcal{D}(A), \forall N \geq 0 .
\end{aligned}
$$

Let $t \geq t_{0}$ be fixed and so that it belongs to the neighbourhood $\mathcal{N}_{X}\left(t_{0} ; a_{0}(\omega)\right)$ of $t_{0}$ defined in Remark 9.3. Notice that this set does not depend on $a_{0}(\omega)$ since (9.13)-(9.15) hold for every $a_{0}(\omega) \in \mathcal{D}(A), \omega \in \Omega$. In the context of Theorem 2.1 with $n=3$ and $\mathbf{X}=\left(A, Y_{0}, Y_{1}\right)$, let us consider mapping $\mathbf{r}: \mathbb{R}^{3} \longrightarrow \mathbb{R}^{3}$ 
defined on a subset of $\mathcal{D}\left(A, Y_{0}, Y_{1}\right)$ such that for $t \geq t_{0}$ fixed, $(t, a(\omega)), \omega \in \Omega$, belongs to the set $\mathcal{N}_{X}^{*}\left(t_{0} ; a_{0}(\omega)\right)$ introduced in Remark 9.3, whose components are given by

$$
\begin{aligned}
& z_{1}=r_{1}\left(a, y_{0}, y_{1}\right)=a \\
& z_{2}=r_{2}\left(a, y_{0}, y_{1}\right)=y_{0} S_{1}^{N}(t ; a)+y_{1} S_{2}^{N}(t ; a), \\
& z_{3}=r_{3}\left(a, y_{0}, y_{1}\right)=y_{1} .
\end{aligned}
$$

Thus, RV $Z_{2}=X_{N}$, i.e., $Z_{2}$ corresponds to the truncated series solution defined by (9.10). Notice that for convenience, dependence on variable $t$ has been omitted in the notation since it has previously been fixed. The inverse of mapping $\mathbf{r}$, denoted by $\mathbf{s}: \mathbb{R}^{3} \longrightarrow \mathbb{R}^{3}$, is given by

$$
\begin{aligned}
& a=s_{1}\left(z_{1}, z_{2}, z_{3}\right)=z_{1}, \\
& y_{0}=s_{2}\left(z_{1}, z_{2}, z_{3}\right)=\frac{z_{2}-z_{3} S_{2}^{N}\left(t ; z_{1}\right)}{S_{1}^{N}\left(t ; z_{1}\right)}, \\
& y_{1}=s_{3}\left(z_{1}, z_{2}, z_{3}\right)=z_{3} .
\end{aligned}
$$

Taking into account (9.12), the absolute value of the Jacobian of this transformation, given by $\left|J_{3}\right|=\frac{1}{\left|S_{1}^{N}\left(t ; z_{1}\right)\right|}=\frac{1}{\left|S_{1}^{N}(t ; a)\right|} \neq 0$, is well-defined. Therefore, applying Theorem 2.1 , the joint PDF of random vector $\left(Z_{1}, Z_{2}, Z_{3}\right)$ is

$$
f_{Z_{1}, Z_{2}, Z_{3}}\left(z_{1}, z_{2}, z_{3}\right)=f_{A, Y_{0}, Y_{1}}\left(z_{1}, \frac{z_{2}-z_{3} S_{2}^{N}\left(t ; z_{1}\right)}{S_{1}^{N}\left(t ; z_{1}\right)}, z_{3}\right)\left|\frac{1}{S_{1}^{N}\left(t ; z_{1}\right)}\right|
$$

Then, marginalizing with respect to $Z_{1}=A$ and $Z_{3}=Y_{1}$, one obtains the $\mathrm{PDF}$ of $\mathrm{RV} Z_{2}=X_{N}$

$$
f_{Z_{2}}\left(z_{2}\right)=\iint_{\mathcal{D}\left(A, Y_{1}\right)} f_{A, Y_{0}, Y_{1}}\left(a, \frac{z_{2}-y_{1} S_{2}^{N}(t ; a)}{S_{1}^{N}(t ; a)}, y_{1}\right)\left|\frac{1}{S_{1}^{N}(t ; a)}\right| \mathrm{d} y_{1} \mathrm{~d} a
$$


As $t \geq t_{0}$ is arbitrary, this latter expression really gives the 1 -PDF of the truncated solution $\mathrm{SP} X_{N}(t)$ to $\operatorname{IVP}(9.1)-(9.2)$

$$
f_{1}^{N}(x, t)=\iint_{\mathcal{D}\left(A, Y_{1}\right)} f_{A, Y_{0}, Y_{1}}\left(a, \frac{x-y_{1} S_{2}^{N}(t ; a)}{S_{1}^{N}(t ; a)}, y_{1}\right)\left|\frac{1}{S_{1}^{N}(t ; a)}\right| \mathrm{d} y_{1} \mathrm{~d} a .
$$

Below, it will be provided conditions under which approximation $f_{1}^{N}(x, t)$ converges to the exact $1-\mathrm{PDF}, f_{1}(x, t)$, i.e.,

$$
\lim _{N \rightarrow+\infty} f_{1}^{N}(x, t)=f_{1}(x, t), \quad \text { for each }(x, t) \in \mathbb{R} \times\left[t_{0},+\infty[\text { fixed, }\right.
$$

being

$$
f_{1}(x, t)=\iint_{\mathcal{D}\left(A, Y_{1}\right)} f_{A, Y_{0}, Y_{1}}\left(a, \frac{x-y_{1} S_{2}(t ; a)}{S_{1}(t ; a)}, y_{1}\right)\left|\frac{1}{S_{1}(t ; a)}\right| \mathrm{d} y_{1} \mathrm{~d} a,
$$

where $S_{1}(t ; a)$ and $S_{2}(t ; a)$ are defined in (9.9).

Notice that, one can formally write

$$
\begin{aligned}
& \lim _{N \rightarrow+\infty} f_{1}^{N}(x, t) \\
& =\lim _{N \rightarrow+\infty} \iint_{\mathcal{D}\left(A, Y_{1}\right)} f_{A, Y_{0}, Y_{1}}\left(a, \frac{x-y_{1} S_{2}^{N}(t ; a)}{S_{1}^{N}(t ; a)}, y_{1}\right)\left|\frac{1}{S_{1}^{N}(t ; a)}\right| \mathrm{d} y_{1} \mathrm{~d} a \\
& \quad \stackrel{(\mathrm{I})}{=} \iint_{\mathcal{D}\left(A, Y_{1}\right)} \lim _{N \rightarrow+\infty}\left(f_{A, Y_{0}, Y_{1}}\left(a, \frac{x-y_{1} S_{2}^{N}(t ; a)}{S_{1}^{N}(t ; a)}, y_{1}\right)\left|\frac{1}{S_{1}^{N}(t ; a)}\right|\right) \mathrm{d} y_{1} \mathrm{~d} a \\
& \stackrel{(\mathrm{II})}{=} \iint_{\mathcal{D}\left(A, Y_{1}\right)} f_{A, Y_{0}, Y_{1}}\left(a, \lim _{N \rightarrow+\infty} \frac{x-y_{1} S_{2}^{N}(t ; a)}{S_{1}^{N}(t ; a)}, y_{1}\right)\left|\frac{1}{\lim _{N \rightarrow+\infty} S_{1}^{N}(t ; a)}\right| \mathrm{d} y_{1} \mathrm{~d} a \\
& =\iint_{\mathcal{D}\left(A, Y_{1}\right)} f_{A, Y_{0}, Y_{1}}\left(a, \frac{x-y_{1} S_{2}(t ; a)}{S_{1}(t ; a)}, y_{1}\right)\left|\frac{1}{S_{1}(t ; a)}\right| \mathrm{d} y_{1} \mathrm{~d} a \\
& =f_{1}(x, t) .
\end{aligned}
$$


As a result, steps (I) and (II) in (9.18) must be justified (9.17). This will be done by assuming that conditions shown in Remark 9.3 are fulfilled for $t \geq t_{0}$ and $x \in \mathbb{R}$. First commutation between the limit as $N \rightarrow+\infty$ and the joint PDF $f_{A, Y_{0}, Y_{1}}$ (step (II) in (9.18)), and secondly, the commutation between the limit as $N \rightarrow+\infty$ and the double integral (step (I) in (9.18)).

Firstly, observe that step (II) in (9.18) is legitimated because of continuity of the joint $\mathrm{PDF} f_{A, Y_{0}, Y_{1}}\left(a, y_{0}, y_{1}\right)$ with respect to its second variable (see hypothesis H1 (see $(9.3)))$.

Let us legitimate step (I) in (9.18). With this goal, Proposition 9.1 will be applied. This leads to assume the following hypothesis hereinafter

H3 :

$$
\mathcal{D}\left(A, Y_{1}\right) \text { is a Lebesgue measurable set of } \mathbb{R}^{2} \text { with finite measure }
$$

such that $\hat{M}=\sup \left\{\left|y_{1}(\omega)\right|:\left(a(\omega), y_{1}(\omega)\right) \in \mathcal{D}\left(A, Y_{1}\right), \omega \in \Omega\right\}<+\infty$.

Let us denote

$$
k_{N}\left(a, y_{1}\right)=f_{A, Y_{0}, Y_{1}}\left(a, \frac{x-y_{1} S_{2}^{N}(t ; a)}{S_{1}^{N}(t ; a)}, y_{1}\right)\left|\frac{1}{S_{1}^{N}(t ; a)}\right|, \quad\left(a, y_{1}\right) \in \mathcal{D}\left(A, Y_{1}\right)
$$

the integrand of (9.16). Then, taking into account Remark 9.3, and particularly the lower bound that appears in $(9.15)$, one gets

$$
k_{N}\left(a, y_{1}\right) \leq \frac{1}{m_{S_{1}}} f_{A, Y_{0}, Y_{1}}\left(a, \frac{x-y_{1} S_{2}^{N}(t ; a)}{S_{1}^{N}(t ; a)}, y_{1}\right), \quad \forall\left(a, y_{1}\right) \in \mathcal{D}\left(A, Y_{1}\right)
$$

Hence, since $f_{A, Y_{0}, Y_{1}}\left(a, y_{0}, y_{1}\right)$ is a PDF, $k_{N}\left(a, y_{1}\right)$ defined by (9.20) is Lebesgue absolutely integrable in $\mathcal{D}\left(A, Y_{1}\right)$, i.e, $k_{N}\left(a, y_{1}\right) \in \mathcal{L}^{1}\left(\mathcal{D}\left(A, Y_{1}\right)\right.$ ) (notice that $k_{N}\left(a, y_{1}\right)$ is non-negative all over its domain). Now, it shall been shown that

$$
k_{N}\left(a, y_{1}\right) \stackrel{\text { uniformly } \mathcal{D}\left(A, Y_{1}\right)}{\underset{N \rightarrow \infty}{\longrightarrow}} k\left(a, y_{1}\right)
$$

being

$$
k\left(a, y_{1}\right)=f_{A, Y_{0}, Y_{1}}\left(a, \frac{x-y_{1} S_{2}(t ; a)}{S_{1}(t ; a)}, y_{1}\right)\left|\frac{1}{S_{1}(t ; a)}\right|
$$


With this goal, let us consider the following inequality, obtained by adding and subtracting the term

$$
\begin{gathered}
f_{A, Y_{0}, Y_{1}}\left(a, \frac{x-y_{1} S_{2}^{N}(t ; a)}{S_{1}^{N}(t ; a)}, y_{1}\right)\left|\frac{1}{S_{1}(t ; a)}\right| \\
\left|k_{N}\left(a, y_{1}\right)-k\left(a, y_{1}\right)\right| \\
=\left|f_{A, Y_{0}, Y_{1}}\left(a, \frac{x-y_{1} S_{2}^{N}(t ; a)}{S_{1}^{N}(t ; a)}, y_{1}\right)\right| \frac{1}{S_{1}^{N}(t ; a)} \mid \\
\quad-f_{A, Y_{0}, Y_{1}}\left(a, \frac{x-y_{1} S_{2}(t ; a)}{S_{1}(t ; a)}, y_{1}\right)\left|\frac{1}{S_{1}(t ; a)}\right| \mid \\
\leq f_{A, Y_{0}, Y_{1}}\left(a, \frac{x-y_{1} S_{2}^{N}(t ; a)}{S_{1}^{N}(t ; a)}, y_{1}\right)\left|\frac{1}{\left|S_{1}^{N}(t ; a)\right|}-\frac{1}{\left|S_{1}(t ; a)\right|}\right| \\
\quad+\frac{1}{\left|S_{1}(t ; a)\right|} \mid f_{A, Y_{0}, Y_{1}}\left(a, \frac{x-y_{1}}{S_{1}^{N}(t ; a)}, y_{1}\right) \\
-f_{A, Y_{0}, Y_{1}}\left(a, \frac{x-y_{1} S_{2}(t ; a)}{S_{1}(t ; a)}, y_{1}\right) \mid .
\end{gathered}
$$

Let $t \geq t_{0}$ and $x \in \mathbb{R}$ be fixed, $N \geq 0$ an arbitrary integer, and consider $(t, a) \equiv(t, a(\omega)) \in \mathcal{N}_{X}^{*}\left(t_{0} ; a_{0}(\omega)\right), \omega \in \Omega$, for all $a_{0}(\omega) \in \mathcal{D}(A)$. Observe that set $\mathcal{N}_{X}^{*}\left(t_{0} ; a_{0}(\omega)\right)$ has been previously introduced in Remark 9.3. Let us make the following identification in Proposition 9.2: $z_{1}=a, z_{2}$ arbitrary, $D=$ $\left\{\left(a, z_{2}\right) \in \mathbb{R}^{2}: z_{2}\right.$ is arbitrary $\}, g_{N}\left(a, z_{2}\right)=g\left(a, z_{2}\right)=1, h_{N}\left(a, z_{2}\right)=S_{1}^{N}(t ; a)$, $h\left(a, z_{2}\right)=S_{1}(t ; a)$ (remember that $t \geq t_{0}$ is fixed and that by Remark 9.3, $h_{N}\left(a, z_{2}\right), h\left(a, z_{2}\right) \neq 0$ for all $\left.\left(a, z_{2}\right) \in D\right) \forall N \geq 0$ and $\forall\left(a, z_{2}\right) \in D, M_{g}=1$, $m_{h}=m_{S_{1}}, M_{h}=M_{S}$ (see (9.15)). Taking into account that $g_{N}\left(a, z_{2}\right)$ converges uniformly to $g\left(a, z_{2}\right)$ on $D, h_{N}\left(a, z_{2}\right)$ converges uniformly to $h\left(a, z_{2}\right)$ on $D$ (see Remark 9.3) and hypothesis H1 (see (9.3)), then for every $\epsilon>0$, there exists $N_{0}$, which depends on $\epsilon$, such that

$$
\left|f_{A, Y_{0}, Y_{1}}\left(a, \frac{x-y_{1} S_{2}^{N}(t ; a)}{S_{1}^{N}(t ; a)}, y_{1}\right)\right| \times\left|\frac{1}{\left|S_{1}^{N}(t ; a)\right|}-\frac{1}{\left|S_{1}(t ; a)\right|}\right| \leq M_{f} \frac{\epsilon}{2 M_{f}}=\frac{\epsilon}{2},
$$

$\forall N \geq N_{0}$, independently of values $\left(a, y_{1}\right)$. 
In order to obtain an analogous result for the second term of the right-hand side of inequality (9.22), let us first apply Proposition 9.3 considering the following identification: $u=a, v=-y_{1}, c=x$ (remember that $x$ is fixed), $l_{N}(a)=$ $S_{2}^{N}(t ; a)$ with $t \geq t_{0}$ fixed and such that $(t, a) \in \mathcal{N}_{X}^{*}\left(t_{0} ; a_{0}(\omega)\right), \omega \in \Omega$, being this set defined in Remark 9.3, $l(a)=S_{2}(t ; a), b_{N}\left(a, y_{1}\right)=x-y_{1} S_{2}^{N}(t ; a)$ and $b\left(a, y_{1}\right)=x-y_{1} S_{2}(t ; a)$. Taking into account that $l_{N}(a)$ converges uniformly to $l(a)$ on $\mathcal{N}_{X}^{*}\left(t_{0} ; a_{0}(\omega)\right)$ (see Remark 9.3) and hypothesis H3 (see (9.19)), one follows

$$
x-y_{1} S_{2}^{N}(t, a) \stackrel{\text { uniformly } \mathcal{D}\left(A, Y_{1}\right)}{N \rightarrow \infty} x-y_{1} S_{2}(t, a), \quad(x, t) \in \mathbb{R} \times\left[t_{0},+\infty[\text { fixed },\right.
$$

for all $\left(a, y_{1}\right) \in \mathcal{D}\left(A, Y_{1}\right)$ such that $(t, a(\omega)) \in \mathcal{N}_{X}^{*}\left(t_{0} ; a_{0}(\omega)\right)$, for every $a_{0}(\omega) \in$ $\mathcal{D}(A), \omega \in \Omega$. Now, applying Proposition 9.2 with $z_{1}=a, z_{2}=y_{1}, g_{N}\left(a, y_{1}\right)=$ $x-y_{1} S_{2}^{N}(t, a), g\left(a, y_{1}\right)=x-y_{1} S_{2}(t ; a)$ (observe that $g_{N}\left(a, y_{1}\right)$ converges uniformly to $g\left(a, y_{1}\right)$ by $\left.(9.24)\right), h_{N}\left(a, y_{1}\right)=S_{1}^{N}(t ; a), h\left(a, y_{1}\right)=S_{1}(t ; a)$ (by Remark 9.3 it is known that $h_{N}\left(a, y_{1}\right)$ converges uniformly to $h\left(a, y_{1}\right)$ and both are nonull), $M_{g}=|x|+\hat{M} \times M_{S}$ (see hypothesis H3 in (9.19)) and $M_{h}=M_{S}$ (see (9.14)), this justifies that

$$
\frac{x-y_{1} S_{2}^{N}(t, a)}{S_{1}^{N}(t, a)} \frac{\text { uniformly } \mathcal{D}\left(A, Y_{1}\right)}{N \rightarrow \infty} \frac{x-y_{1} S_{2}(t, a)}{S_{1}(t, a)},(x, t) \in \mathbb{R} \times\left[t_{0},+\infty[\text { fixed. }\right.
$$

Now, let us apply Proposition 9.4 with the following identification: $z_{1}=a$, $z_{2}=y_{1}, D \subset \mathbb{R}^{2}$ the set of $\left(a, y_{1}\right) \in \mathcal{D}\left(A, Y_{1}\right)$ such that $\left(t, a(\omega) \in \mathcal{N}_{X}^{*}\left(t_{0} ; a(\omega)\right)\right.$ for every $a_{0}(\omega) \in \mathcal{D}(A), \omega \in \Omega$,

$$
\gamma_{N}\left(a, y_{1}\right)=\frac{x-y_{1} S_{2}^{N}(t ; a)}{S_{1}^{N}(t ; a)}, \quad \gamma\left(a, y_{1}\right)=\frac{x-y_{1} S_{2}(t ; a)}{S_{1}(t ; a)}
$$

$\phi \equiv f_{A, Y_{0}, Y_{1}}$ and

$$
\begin{aligned}
& \psi_{N}\left(a, y_{1}\right)=f_{A, Y_{0}, Y_{1}}\left(a, \frac{x-y_{1} S_{2}^{N}(t ; a)}{S_{1}^{N}(t ; a)}, y_{1}\right) \\
& \psi\left(a, y_{1}\right)=f_{A, Y_{0}, Y_{1}}\left(a, \frac{x-y_{1} S_{2}(t ; a)}{S_{1}(t ; a)}, y_{1}\right) .
\end{aligned}
$$


Notice that, by $(9.25), \gamma_{N}\left(a, y_{1}\right)$ converges uniformly to $\gamma\left(a, y_{1}\right)$ in $D$, and by hypothesis $\mathrm{H} 1$ (see $(9.3)), \phi$ is a continuous mapping with respect to $y_{0}$. Then, applying Proposition 9.4 one can assure that for every $\epsilon>0$, there exists $N_{0}$, which depends on $\epsilon$, such that

$$
\begin{aligned}
& \frac{1}{\left|S_{1}(t ; a)\right|}\left|f_{A, Y_{0}, Y_{1}}\left(a, \frac{x-y_{1} S_{2}^{N}(t ; a)}{S_{1}^{N}(t ; a)}, y_{1}\right)-f_{A, Y_{0}, Y_{1}}\left(a, \frac{x-y_{1} S_{2}(t ; a)}{S_{1}(t ; a)}, y_{1}\right)\right| \\
& <\frac{1}{m_{S_{1}}} \frac{m_{S_{1}} \epsilon}{2}=\frac{\epsilon}{2}, \quad \forall N \geq N_{0},
\end{aligned}
$$

independently of values $\left(a, y_{1}\right)$. Observe that in (9.26) it has been used that from (9.15) one gets $0<m_{S_{1}} \leq\left|S_{1}(t ; a)\right|$ in the set considered. Therefore, from $(9.22),(9.23)$ and $(9.26)$, one deduces that for every $\epsilon>0$ there exists $N_{0}$, which depends on $\epsilon$ such that

$$
\left|k_{N}\left(a, y_{1}\right)-k\left(a, y_{1}\right)\right|<\epsilon, \quad \forall N \geq N_{0}
$$

independently of values $\left(a, y_{1}\right)$. Hence, (9.21) is proved.

Summarizing, the following result is established.

Theorem 9.2 Let us consider IVP (9.1)-(9.2) and assume that

i) The joint probability density function $f_{A, Y_{0}, Y_{1}}\left(a, y_{0}, y_{1}\right)$ of random input vector $\left(A, Y_{0}, Y_{1}\right)$ satisfies hypothesis $H 1$ (see (9.3)).

ii) Coefficients $p(t ; A)$ and $q(t ; A)$ are stochastic processes satisfying hypothesis H2 (see (9.4)).

iii) Codomain of random vector $\left(A, Y_{1}\right), \mathcal{D}\left(A, Y_{1}\right)$, verifies hypothesis $H 3$ (see (9.19)).

Let $S_{1}^{N}(t ; a)$ and $S_{2}^{N}(t ; a)$ be truncated random power series solutions of the form (9.9) to IVP (9.1)-(9.2). Then, $f_{1}^{N}(x, t)$ defined by (9.16) is the first probability density function of the approximation (9.10) of solution stochastic process $X(t)$ given by (9.8) to IVP (9.1)-(9.2). Furthermore, for each $(x, t)$, $f_{1}^{N}(x, t)$ converges to the first probability density function $f_{1}(x, t)$ of $X(t)$. 


\subsection{Numerical examples}

This section is addressed to show two examples where previous theoretical results are illustrated. In order to show the capability of the approach to deal with a variety of statistical distributions, in the first example it will be considered that input parameters have a joint PDF, whereas in the second example input parameters are assumed to be independent RVs with different probability distributions.

Example 9.1 Let us consider IVP

$$
\left.\begin{array}{cl}
\ddot{X}(t)+A X(t) & =0, \quad t>0, \\
X(0) & =Y_{0}, \\
\dot{X}(0) & =Y_{1},
\end{array}\right\}
$$

where $\left(A, Y_{0}, Y_{1}\right)$ is a random vector with a multivariate Gaussian distribution, $\left(A, Y_{0}, Y_{1}\right) \sim \mathrm{N}_{\mathrm{T}}(\boldsymbol{\mu} ; \boldsymbol{\Sigma})$, truncated on domain $\mathrm{T}=[-2.4,4.4] \times[-3,9] \times$ $[0.6,7.4] \subset \mathbb{R}^{3}$, i.e., $a(\omega) \in[-2.4,4.4], y_{0}(\omega) \in[-3,9]$ and $y_{1}(\omega) \in[0.6,7.4]$, $\omega \in \Omega$, being $\boldsymbol{\mu}$ the mean vector and $\boldsymbol{\Sigma}$ the variance-covariance matrix given by

$$
\boldsymbol{\mu}=(1,2,4)^{\top}, \quad \boldsymbol{\Sigma}=\left(\begin{array}{ccc}
1 / 2 & -1 / 4 & 1 / 3 \\
-1 / 4 & 2 / 3 & 1 / 5 \\
1 / 3 & 1 / 5 & 1 / 2
\end{array}\right)
$$

In Figures 9.1-9.3, the exact 1-PDF, $f_{1}(x, t)$, and the approximations, $f_{1}^{N}(x, t)$, of solution SP to IVP (9.1) have been plotted for different values of truncation order $N$ at time instants $t \in\{1,1.5,2\}$. From these graphical representations, one observes that $f_{1}^{N}(x, t)$ tends to $f_{1}(x, t)$ as $N$ increases, for every value of t. Moreover, excellent approximations are achieved by considering a few terms. In this regard, for instance, in Figure 9.1 one can observe that $f_{1}^{N}(x, t), N=2$, approximately coincides with the exact function $f_{1}(x, t)$ at $t=1$. The same can be said for the approximations $f_{1}^{N}(x, 1.5)$ and $f_{1}^{N}(x, 2)$, taking $N=3$ and $N=$ 4, (see Figures 9.2 and 9.3, respectively). Naturally, the further away from the origin is $t$, the higher order $N$ is required to enhance the approximation. To facilitate the graphical assessment of approximations, function $f_{1}^{N}(x, t)$, for the corresponding greatest value of $N$ and the corresponding $t$, has been plotted together with the exact 1-PDF, $f_{1}(x, t)$, in the right-hand side of Figures 9.19.3. In all cases one can see that $f_{1}^{N}(x, t)$ matches the exact 1-PDF $f_{1}(x, t)$. 

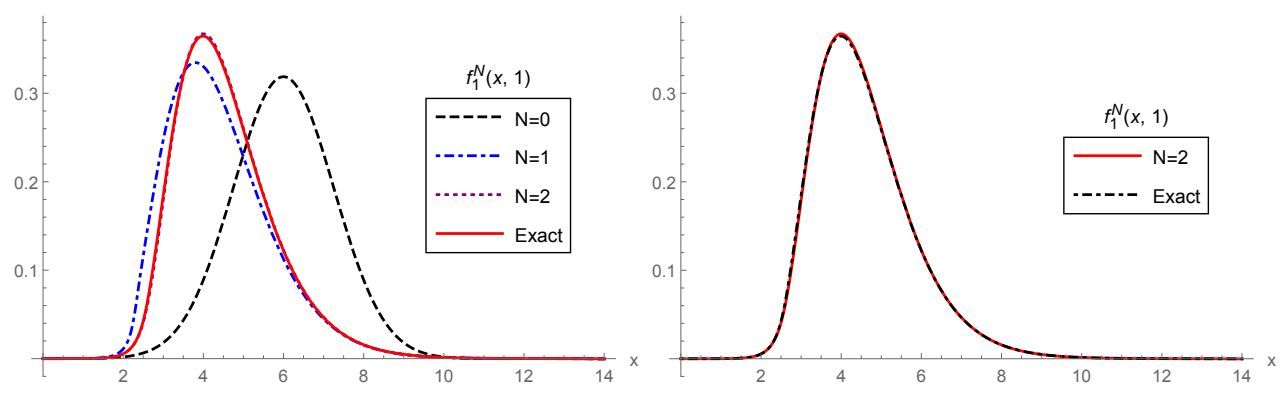

Figure 9.1: Left: Comparison of approximations, $f_{1}^{N}(x, t)$, of the 1-PDF of solution SP to random IVP (9.1) taking as series order truncation $N \in\{0,1,2\}$ and the corresponding exact 1-PDF $f_{1}(x, t)$ at $t=1$. Right: For ease of comparison, function $f_{1}^{N}(x, 1)$, with $N=2$, has been plotted together with the exact 1-PDF $f_{1}(x, 1)$.
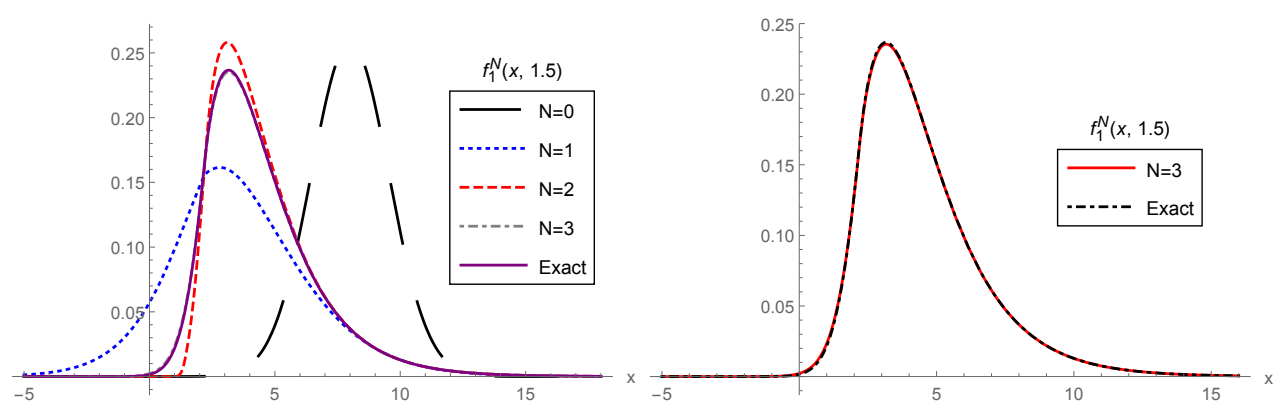

Figure 9.2: Left: Comparison of approximations, $f_{1}^{N}(x, t)$, of the 1-PDF of solution SP to random IVP (9.1) taking as series order truncation $N \in\{0,1,2,3\}$ and the corresponding exact 1-PDF $f_{1}(x, t)$ at $t=1.5$. Right: For ease of comparison, function $f_{1}^{N}(x, 1.5)$, with $N=3$, has been plotted together with the exact 1-PDF $f_{1}(x, 1.5)$. 

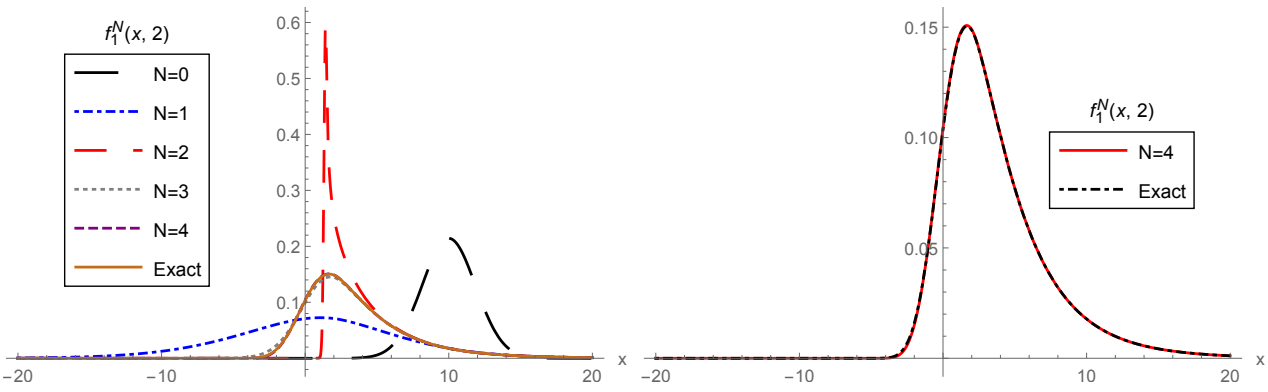

Figure 9.3: Left: Comparison of approximations, $f_{1}^{N}(x, t)$, of the 1-PDF of solution SP to random IVP (9.1) taking as series order truncation $N \in\{0,1,2,3,4\}$ and the corresponding exact 1-PDF $f_{1}(x, t)$ at $t=2$. Right: For ease of comparison, function $f_{1}^{N}(x, 2)$, with $N=4$, has been plotted together with the exact $1-\mathrm{PDF} f_{1}(x, 2)$.

From Figures 9.1-9.3 it is observed that differences between approximations $f_{1}^{N}(x, t)$ and the exact PDF $f_{1}(x, t)$ become smaller as truncation order $N$ increases. To assess the quality of approximations, in Table 9.1 the figures are shown, for the following error measure

$$
e_{N}=\int_{-\infty}^{+\infty}\left|f_{1}^{N}(x, t)-f_{1}(x, t)\right| \mathrm{d} x, \quad N \geq 0, t \geq 0 \text { fixed }
$$

taking as stopping criterion for the tolerance error $10^{-2}$.

\begin{tabular}{cccccc}
\hline$e_{N}$ & $N=0$ & $N=1$ & $N=2$ & $N=3$ & $N=4$ \\
\hline \hline$t=1.0$ & 0.963814 & 0.144837 & 0.008689 & - & - \\
\hline$t=1.5$ & 1.42046 & 0.418365 & 0.083541 & 0.005512 & - \\
\hline$t=2.0$ & 1.6199 & 0.672689 & 0.539773 & 0.035664 & 0.003647 \\
\hline
\end{tabular}

Table 9.1: Error measure $e_{N}$ defined by (9.27) for different time instants, $t \in\{1,1.5,2\}$, and series truncation orders, $N \in\{0,1,2,3,4\}$ in Example 9.1. For every $t$, it has been taken $10^{-2}$ as stopping criterion for the tolerance error to $e_{N}$, then values up to the error is for the first time less than $10^{-2}$ have just collected. Otherwise, error is still less than $10^{-2}$ and it has been denoted by means of an hyphen - . 
Example 9.2 Let us consider the Airy IVP

$$
\left.\begin{array}{cl}
\ddot{X}(t)+A t X(t) & =0, \quad t>0, \\
X(0) & =Y_{0}, \\
\dot{X}(0) & =Y_{1},
\end{array}\right\}
$$

where $A$ is an Uniform $R V, A \sim \mathrm{U}([1,2]), Y_{0}$ is an Exponential $R V$ of parameter $\lambda=1 / 2, Y_{0} \sim \operatorname{Exp}(1 / 2)$ and $Y_{1}$ is a Beta $R V$ of parameters $\alpha=2$ and $\beta=3, Y_{1} \sim \operatorname{Be}(2 ; 3)$. Hereinafter, $A, Y_{0}$ and $Y_{1}$ are assumed to be independent $R V$ s. To illustrate the capability of the approach to approximate the 1-PDF of solution SP to IVP (9.28), approximations, $f_{1}^{N}(x, t)$, for different values of $N$ and time instants $t \in\{0.5,1.5 .2 .5\}$, have been plotted (see Figures 9.4-9.6). In Figure 9.4, one can see that approximations corresponding to $N=3$ and $N=4$ match. To facilitate the graphical assessment of approximations, functions $f_{1}^{N}(x, 1.5)$, with $N \in\{2,3,4\}$ and $f_{1}^{N}(x, 2.5)$, with $N \in\{4,5,6\}$ have been magnified on the right-hand side of Figures 9.5 and 9.6, respectively. From both graphical representations, one can see that the approximations $\left\{f_{1}^{3}(x, 1.5), f_{1}^{4}(x, 1.5)\right\}$ and $\left\{f_{1}^{5}(x, 2.5), f_{1}^{6}(x, 2.5)\right\}$ match, respectively.

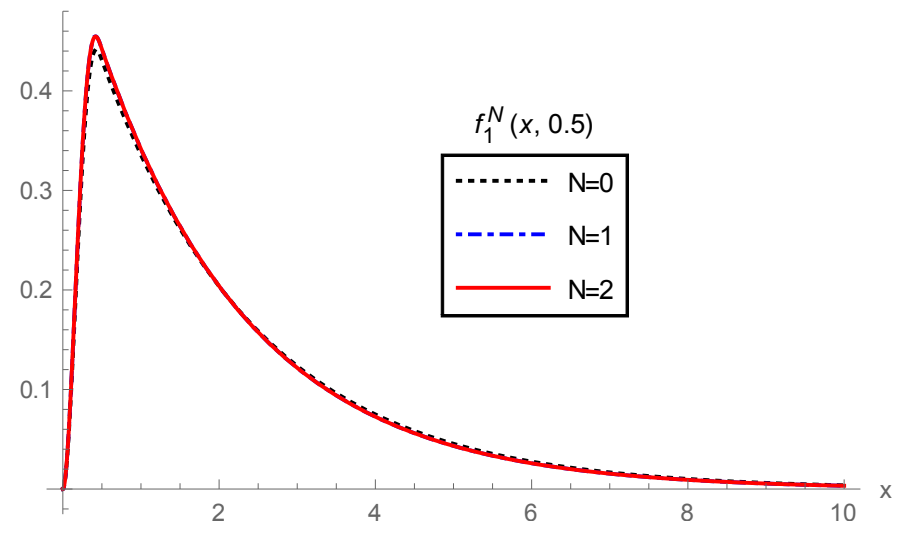

Figure 9.4: Approximations, $f_{1}^{N}(x, 0.5)$, of the 1-PDF of solution SP to random IVP (9.28) taking as series order truncation $N \in\{0,1,2\}$.

From these graphical representations, one observe that differences between approximations $f_{1}^{N+1}(x, t)$ and $f_{1}^{N}(x, t)$ are smaller as $N$ increases. As it also happens in the deterministic scenario, when classical Fröbenius method is applied, one observes that very good approximations are achieved even for small 

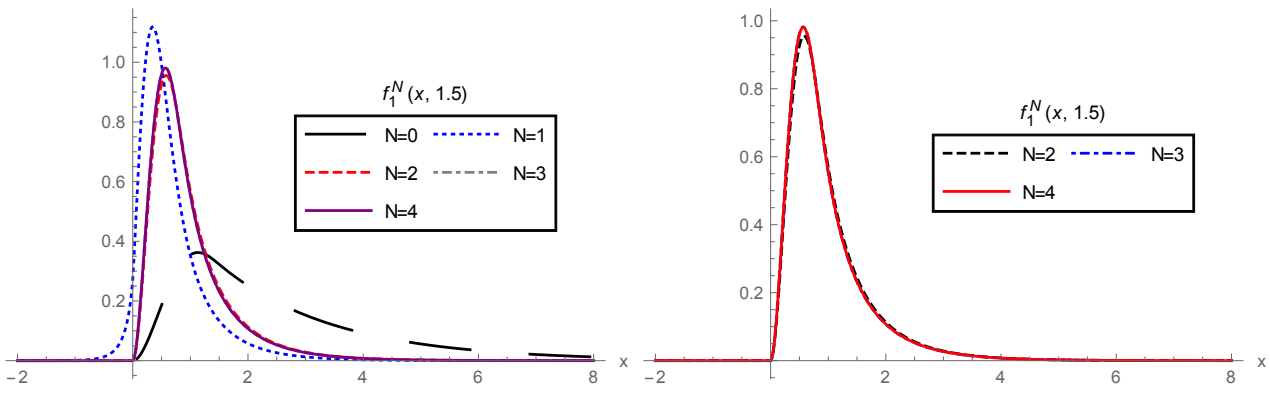

Figure 9.5: Left: Approximations, $f_{1}^{N}(x, 1.5)$, of the 1-PDF of solution SP to random IVP (9.28) taking as series order truncation $N \in\{0,1,2,3,4\}$. Right: To facilitate the graphical assessment of approximations, the functions $f_{1}^{N}(x, 1.5)$, with $N \in\{2,3,4\}$, have been magnified.

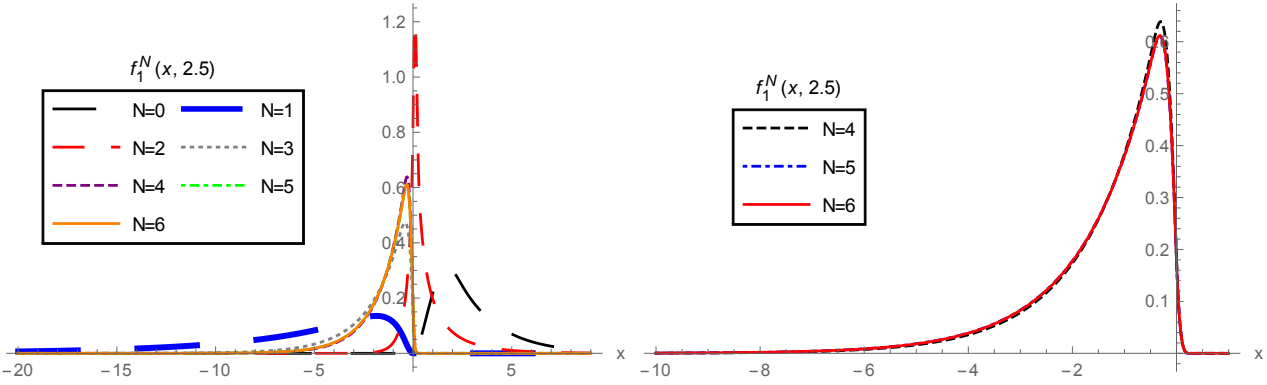

Figure 9.6: Left: Approximations, $f_{1}^{N}(x, 2.5)$, of the 1-PDF of solution SP to random IVP (9.28) taking as series order truncation $N \in\{0,1,2,3,4,5,6\}$. Right: To facilitate the graphical assessment of approximations, functions $f_{1}^{N}(x, 2.5)$, with $N \in\{4,5,6\}$, have been magnified.

values of the truncation order $N$. This assertion is numerically confirmed in Table 9.2 where the following error measure

$$
\hat{e}_{N}=\int_{-\infty}^{+\infty}\left|f_{1}^{N+1}(x, t)-f_{1}^{N}(x, t)\right| \mathrm{d} x, \quad N \geq 0, t \geq 0 \text { fixed }
$$

is reported taking as stopping criterion for the tolerance error $10^{-2}$. 


\begin{tabular}{ccccccc}
\hline$\hat{e}_{N}$ & $N=0$ & $N=1$ & $N=2$ & $N=3$ & $N=4$ & $N=5$ \\
\hline \hline$t=0.5$ & 0.024571 & 0.000159 & - & - & - & - \\
\hline$t=1.5$ & 1.25224 & 0.514115 & 0.036846 & 0.001671 & - & - \\
\hline$t=2.5$ & 1.98864 & 1.86826 & 1.61559 & 0.227534 & 0.033886 & 0.002859 \\
\hline
\end{tabular}

Table 9.2: Error measure $\hat{e}_{N}$ defined by (9.29) for different time instants, $t \in\{0.5,1.5,2.5\}$, and series truncation orders, $N \in\{0,1,2,3,4,5\}$ in Example 9.2. For every $t, 10^{-2}$ has been taken as stopping criterion for the tolerance error to $\hat{e}_{N}$, then values up to error is for the first time less that $10^{-2}$ have just collected. Otherwise, error is still less than $10^{-2}$ and it has been denoted by means of an hyphen - .

\subsection{Conclusions}

Many important differential equations belonging to the Mathematical Physics and other areas related to Applied Mathematics are formulated through secondorder linear differential equations whose coefficients are analytic about regular points. Taking into account the physical meaning of these equations, it is natural to consider randomness in their coefficients and initial conditions. In this chapter approximations to the first probability density function of solution stochastic process of such significant equations under very general hypotheses have been computed. The most important issues of our contribution are, firstly, statistical dependence among input random variables has been considered. This approach enables to deal with the more standard scenario, where input parameters are assumed to be independent random variables, as a particular case. Secondly, uncertainty has been considered in very general point of view since a wide range of probability distributions are allowed as input parameters, including Beta, Exponential, Uniform, Gamma, Gaussian, etc. This is an important difference against the Itô-type approach, where uncertainty into stochastic differential equations is formulated through the differential of brownian motion or Wiener process, usually referred to as, white noise, which is a Gaussian distribution. Thirdly, it has been pointed out that an important advantage of computing approximations of the first probability density function of solution stochastic process allows for computing approximations of mean and variance just as merely particular cases. Therefore, this contribution addresses a quite general problem. 
Chapter 10

\section{On the computation of the first probability density function to random non-autonomous} first-order linear homogeneous differential equations

This chapter is devoted to construct approximations of the probability density function of non-autonomous first-order homogeneous linear random differential equation, where initial condition and diffusion coefficient are assumed to be a random variable and a stochastic process, respectively. Random Variable Transformation technique and Karhunen-Loève expansion will be combined to construct reliable approximations under general conditions. Several numerical examples illustrate theoretical findings. 


\subsection{Introduction}

Throughout this chapter non-autonomous first-order linear random differential equations will be considered. Down below, some illustrative examples that are aimed to motivate the interest in studying this kind of IVPs will be exhibited. Indeed, important results, well-known in the deterministic framework, are satisfied in the random scenario only under restrictive assumptions. For instance, considering the autonomous first-order linear random differential equation with deterministic initial condition

$$
\dot{X}(t, \omega)=A(\omega) X(t, \omega), \quad t \geq 0 ; \quad X(0, \omega)=1, \quad \omega \in \Omega,
$$

where $A \equiv A(\omega)$ is a $2-\mathrm{RV}$, it can be shown that the extension to the random scenario of the classical existence and uniqueness Picard's theorem is satisfied if, and only if, $A$ is bounded almost surely [85, p.119], [86]. As a consequence, this important result is not applicable when $A$ assumes a Gaussian or a Poisson distribution, for instance. As a second illustrative example involving the computation of the expectation of solution SP to non-autonomous linear RDEs, let us consider random IVP

$$
\dot{X}(t, \omega)=A(t, \omega) X(t, \omega)+B(t, \omega), \quad X\left(t_{0}, \omega\right)=X_{0}(\omega), \quad \omega \in \Omega,
$$

where $A(t, \omega)$ and $B(t, \omega)$ are second-order SPs $\left(\mathbb{E}\left[(A(t, \omega))^{2}\right]\right.$ and $\mathbb{E}\left[(B(t, \omega))^{2}\right]$ are finite for all $t)$ and $A_{0}(\omega)$ is a second-order RV. If $\mathbb{E}[\cdot]$ denotes the expectation operator, then it can be shown that the mean of solution, $\mu_{X}(t)=$ $\mathbb{E}[X(t, \omega)]$, does not satisfy the corresponding averaged ordinary differential equation

$$
\frac{\mathrm{d} \mu_{X}(t)}{\mathrm{d} t} \neq \mathbb{E}[A(t, \omega)] \mu_{X}(t)+\mathbb{E}[B(t, \omega)], \quad \mu_{X}\left(t_{0}\right)=\mathbb{E}\left[X_{0}(\omega)\right] .
$$

Instead the computation of the expectation $\mu_{X}(t)$ is more involved (see, [85, Chapter 8$]$ and $[40$, p. 66]). The earlier examples illustrate the challenges when dealing with both theoretical and practical aspects regarding linear random differential equations.

The computation of the 1-PDF of solution SP for linear random differential equation has been recently undertaken in [16], where inputs parameters were considered RVs rather than deterministic constants. In this chapter, it will 
be studied the non-autonomous case for the homogeneous linear random differential equation whose initial condition is assumed to be a RV. Specifically, hereinafter the following random IVP will be considered

$$
\left.\begin{array}{l}
\dot{X}(t, \omega)=A(t, \omega) X(t, \omega), \quad t \in \mathcal{T}=\left[t_{0}, T\right] \subset \mathbb{R}, \\
X\left(t_{0}, \omega\right)=X_{0}(\omega) .
\end{array}\right\}
$$

The main goal of this chapter is to obtain the 1-PDF, $f_{1}(x, t)$, of solution $\mathrm{SP}$, $X(t, \omega)$, to random IVP $(10.1)$. To achieve this objective, it will combined the application of RVT technique (see Theorem 2.1) and Karhunen-Loève expansion (KLE).

The chapter is organized as follows. In Section 10.2 both the notation, that will be used in this chapter, and the definition and some results regarding the KLE will be established. The aim of Section 10.3 is twofold, first to compute the 1-PDF, $f_{1}^{N}(x, t)$, of the truncated solution SP, $X_{N}(t, \omega)$, secondly, to provide sufficient conditions in order to guarantee the convergence of the 1-PDF, $f_{1}^{N}(x, t)$, to exact solution SP, $X(t, \omega)$, as $N \rightarrow+\infty$. In Section 10.4, two numerical examples will be shown to illustrate the theoretical results established in Section 10.3. Conclusions are drawn in Section 10.5.

\subsection{Notation and auxiliary results}

It is important to point out that the rigorous analysis of stochastic differential equations and random differential equations usually takes place in the Hilbert space $\mathrm{L}^{2}\left(\Omega, \mathrm{L}^{2}(\mathcal{T}, \mathrm{H})\right)$ of mean square integrable SPs, that are valued on a Hilbert space $\left(\mathrm{H},\langle\cdot, \cdot\rangle_{\mathrm{H}}\right)$, and these SPs are defined over an underlying complete probability space $(\Omega, \mathcal{A}, \mathbb{P})$. Let us introduce the mathematical ingredients that will be required to develop rigorously the working context:

- The Hilbert space $\left(H,\langle\cdot, \cdot\rangle_{\mathrm{H}}\right)$ previously introduced. For reasons that will be apparent later, elements of $\left(\mathrm{H},\langle\cdot, \cdot\rangle_{\mathrm{H}}\right)$ will be denoted by $X(t, \omega)$. Then,

$$
\mathrm{H}=\left\{X(t, \omega):\|X(t, \omega)\|_{\mathrm{H}}=\sqrt{\langle X(t, \omega), X(t, \omega)\rangle_{\mathrm{H}}}<+\infty,(t, \omega) \in \mathcal{T} \times \Omega\right\}
$$

- The Hilbert space $\left(\mathrm{L}^{2}(\mathcal{T}, \mathrm{H}),\langle\cdot, \cdot\rangle_{\mathrm{L}^{2}(\mathcal{T}, \mathrm{H})}\right), \mathcal{T} \subset \mathbb{R}$, defined as 


$$
\mathrm{L}^{2}(\mathcal{T}, \mathrm{H})=\left\{X(\cdot, \omega): \mathcal{T} \longrightarrow \mathrm{H}:\|X(\cdot, \omega)\|_{\mathrm{L}^{2}(\mathcal{T}, \mathrm{H})}<+\infty\right\}
$$

where

$$
\langle X(\cdot, \omega), Y(\cdot, \omega)\rangle_{\mathrm{L}^{2}(\mathcal{T}, \mathrm{H})}=\int_{\mathcal{T}}\langle X(t, \omega), Y(t, \omega)\rangle_{\mathrm{H}} \mathrm{d} t
$$

and

$$
\begin{aligned}
\|X(\cdot, \omega)\|_{\mathrm{L}^{2}(\mathcal{T}, \mathrm{H})} & =+\sqrt{\langle X(\cdot, \omega), X(\cdot, \omega)\rangle_{\mathrm{L}^{2}(\mathcal{T}, \mathrm{H})}} \\
& =\left(\int_{\mathcal{T}}\langle X(t, \omega), X(t, \omega)\rangle_{\mathrm{H}} \mathrm{d} t\right)^{1 / 2} \\
& =\left(\int_{\mathcal{T}}\left(\|X(t, \omega)\|_{\mathrm{H}}\right)^{2} \mathrm{~d} t\right)^{1 / 2}<+\infty .
\end{aligned}
$$

From these ingredients, one defines the Hilbert space

$$
\mathrm{L}^{2}\left(\Omega, \mathrm{L}^{2}(\mathcal{T}, \mathrm{H})\right)=\left\{X: \mathcal{T} \times \Omega \longrightarrow \mathrm{L}^{2}(\mathcal{T}, \mathrm{H}):\|X\|_{\mathrm{L}^{2}\left(\Omega, \mathrm{L}^{2}(\mathcal{T}, \mathrm{H})\right)}<+\infty\right\}
$$

with the following inner product

$$
\begin{aligned}
\langle X, Y\rangle_{\mathrm{L}^{2}\left(\Omega, \mathrm{L}^{2}(\mathcal{T}, \mathrm{H})\right)} & =\int_{\Omega}\langle X(t, \omega), Y(t, \omega)\rangle_{\mathrm{L}^{2}(\mathcal{T}, \mathrm{H})} \mathrm{d} \mathbb{P}(\omega) \\
& =\int_{\Omega} \int_{\mathcal{T}}\langle X(t, \omega), Y(t, \omega)\rangle_{\mathrm{H}} \mathrm{d} t \mathrm{~d} \mathbb{P}(\omega) \\
& =\int_{\mathcal{T}} \int_{\Omega}\langle X(t, \omega), Y(t, \omega)\rangle_{\mathrm{H}} \mathrm{d} \mathbb{P}(\omega) \mathrm{d} t \\
& =\int_{\mathcal{T}} \mathbb{E}\left[\langle X(t, \omega), Y(t, \omega)\rangle_{\mathrm{H}}\right] \mathrm{d} t,
\end{aligned}
$$

and norm 


$$
\|X\|_{\mathrm{L}^{2}\left(\Omega, \mathrm{L}^{2}(\mathcal{T}, \mathrm{H})\right)}=\left(\int_{\mathcal{T}} \mathbb{E}\left[\left(\|X(t, \omega)\|_{\mathrm{H}}\right)^{2}\right] \mathrm{d} t\right)^{1 / 2}<+\infty
$$

where the Fubbini's theorem has been applied to express both, the inner product and the norm, in terms of expectation operator. So far, elements $x$ and $x(t, \omega)$, for the sake of convenience, will be written indistinctly.

As throughout this chapter real RVs and SPs will be considered, $\mathrm{H}=\mathbb{R}$ will be taken endowed with the standard inner product $\langle x, y\rangle_{\mathbb{R}}=x y$, being $x, y \in \mathbb{R}$. In this case $\mathrm{L}^{2}\left(\Omega, \mathrm{L}^{2}(\mathcal{T}, \mathbb{R})\right)$ is usually denoted as $\mathrm{L}^{2}\left(\Omega, \mathrm{L}^{2}(\mathcal{T})\right)$. Moreover, it is assumed that $\mathcal{A}=\mathcal{B}(\mathbb{R})$ is the Borel $\sigma$-algebra on $\mathbb{R}$. Therefore, this work is based on the Hilbert space $\mathrm{L}^{2}\left(\Omega, \mathrm{L}^{2}(\mathcal{T})\right)$ with the inner product

$$
\langle X, Y\rangle_{\mathrm{L}^{2}\left(\Omega, \mathrm{L}^{2}(\mathcal{T})\right)}=\int_{\mathcal{T}} \mathbb{E}[X(t, \omega) Y(t, \omega)] \mathrm{d} t
$$

and whose elements are SPs such that $\int_{\mathcal{T}} \mathbb{E}\left[(X(t, \omega))^{2}\right] \mathrm{d} t<+\infty$.

In practice, an important case, that will be considered throughout the subsequent development, is when $\mathcal{T}$ is a bounded and closed interval of the real line, $\mathcal{T}=\left[t_{0}, T\right] \subset \mathbb{R}$. In that case, 2-RVs, that is $\mathrm{RVs} X(\omega)$ with finite variance (and hence $\left.\mathbb{E}\left[(X(\omega))^{2}\right]<+\infty\right)$, are obviously elements of the Hilbert space $\mathrm{L}^{2}\left(\Omega, \mathrm{L}^{2}(\mathcal{T})\right)$. These RVs are interpreted as constant SPs. In dealing with sequences of $2-R V s$, the above inner product defines a norm whose associate convergence is usually referred to as m.s. convergence. Apart from this convergence, the study of stochastic differential equations and random differential equations can be developed considering another types of stochastic convergences such as almost surely convergence, convergence in probability and convergence in distribution, and using the relationship among them as well.

Moreover, as it was pointed before, in this chapter KLE will be used. KLE is a type-Fourier series method that allows to represent diffusion SP in (10.1), $A(t, \omega)$, as a function of a denumerable set of 2-RVs $\left\{\xi_{i}(\omega): i \geq 1\right\}$ such that they have zero mean $\left(\mathbb{E}\left[\xi_{i}(\omega)\right]=0\right)$, unit variance $\left(\mathbb{V}\left[\xi_{i}(\omega)\right]=1\right)$ and are pairwise uncorrelated $\left(\mathbb{E}\left[\xi_{i}(\omega) \xi_{j}(\omega)\right]=0\right.$ if $\left.i \neq j\right)$. In other words, $\left\{\xi_{i}(\omega): i \geq\right.$ $1\}$ are such that $\mathbb{E}\left[\xi_{i}(\omega)\right]=0$ and $\mathbb{E}\left[\xi_{i}(\omega) \xi_{j}(\omega)\right]=\delta_{i j}$, where $\delta_{i j}$ denotes the standard Kronecker delta function.

Theorem 10.1 ( $\mathrm{L}^{2}$ convergence of Karhunen-Loève) /61, p.202/ Consider a mean square integrable continuous time stochastic process $X \equiv\{X(t, \omega)$ : 
$t \in \mathcal{T}, \omega \in \Omega\}$, i.e., $X \in \mathrm{L}^{2}\left(\Omega, \mathrm{L}^{2}(\mathcal{T})\right)$ being $\mu_{X}(t)$ and $c_{X}(s, t)$ its mean and covariance functions, respectively. Then,

$$
X(t, \omega)=\mu_{X}(t)+\sum_{j=1}^{\infty} \sqrt{\nu_{j}} \phi_{j}(t) \xi_{j}(\omega), \quad \omega \in \Omega,
$$

where, this sum converges in $\mathrm{L}^{2}\left(\Omega, \mathrm{L}^{2}(\mathcal{T})\right)$,

$$
\xi_{j}(\omega):=\frac{1}{\sqrt{\nu_{j}}}\left\langle X(t, \omega)-\mu_{X}(t), \phi_{j}(t)\right\rangle_{\mathrm{L}^{2}(\mathcal{T})}
$$

$\left\{\left(\nu_{j}, \phi_{j}(t)\right): j \geq 1\right\}$ denote, respectively, the eigenvalues with $\nu_{1} \geq \nu_{2} \geq \cdots>0$ and eigenfunctions of the following integral operator $\mathfrak{C}$

$$
(\mathfrak{C} f)(t):=\int_{\mathcal{T}} c_{X}(s, t) f(s) \mathrm{d} s, \quad f \in \mathrm{L}^{2}(\mathcal{T})
$$

associated to the covariance function $c_{X}(s, t)$. Random variables $\xi_{j}(\omega)$ have zero mean, unit variance and are pairwise uncorrelated. Furthermore, if $X(t, \omega)$ is Gaussian, then $\xi_{j}(\omega) \sim \mathrm{N}(0 ; 1)$ are i.i.d.

To keep the computational burden feasible, later to apply RVT technique to compute approximations of the $1-\mathrm{PDF}, f_{1}(x, t)$, it will be needed to consider the $N$-truncation of the infinite sum (10.2)

$$
X_{N}(t, \omega)=\mu_{X}(t)+\sum_{j=1}^{N} \sqrt{\nu_{j}} \phi_{j}(t) \xi_{j}(\omega), \quad \omega \in \Omega .
$$

\subsection{Computing the 1-PDF of truncated solution stochastic process}

In this section the 1-PDF of truncated solution SP of IVP (10.1) will be obtained. Notice that in IVP (10.1) it will be considered

H1 : $X_{0}(\omega)$ is a second-order RV and $A(t, \omega) \in \mathrm{L}^{2}\left(\Omega, \mathrm{L}^{2}(\mathcal{T})\right)$, 
satisfying certain additional conditions that will be specified later. As it is assumed that $X_{0}(\omega)$ is a $2-\mathrm{RV}\left(\mathbb{E}\left[\left(X_{0}(\omega)\right)^{2}\right]=k_{0}<+\infty\right)$ and $\mathcal{T}=\left[t_{0}, T\right]$ has finite volume, then $X_{0}(\omega) \in \mathrm{L}^{2}\left(\Omega, \mathrm{L}^{2}(\mathcal{T})\right)$

$$
\left\|X_{0}\right\|_{\mathrm{L}^{2}\left(\Omega, \mathrm{L}^{2}(\mathcal{T}, \mathrm{H})\right)}=\left(\int_{\mathcal{T}} \mathbb{E}\left[\left(\left\|X_{0}(\omega)\right\|_{\mathrm{H}}\right)^{2}\right] \mathrm{d} t\right)^{1 / 2}<\sqrt{k_{0}\left(T-t_{0}\right)}<+\infty .
$$

It is known that the exact closed solution SP to random IVP (10.1) is

$$
X(t, \omega)=X_{0}(\omega) \operatorname{Exp}\left[\int_{t_{0}}^{t} A(s, \omega) \mathrm{d} s\right], \quad \omega \in \Omega
$$

It is important to note that given a $\mathrm{SP}$, say $A(t, \omega)$, in general nothing is known about the probabilistic distribution of the SP, $\hat{A}(t, \omega)=\int_{0}^{t} A(s, \omega) \mathrm{d} s$. An exception is when $A(t, \omega)$ is a Gaussian SP. In that case, it can be proved that $\hat{A}(t, \omega)$ is also Gaussian, see [85, Theorem 4.64, p.112]. This chapter is focused in the determination of the 1-PDF of the SP (10.4) in the general case that the SP $A(t, \omega)$ has an arbitrary probabilistic distribution (hence no Gaussian in general), KLE and RVT techniques will be combined to give an answer to this interesting question under mild conditions.

The analysis will be carried out assuming that initial condition $X_{0}(\omega)$ is a RV such as, for every $t \in \mathcal{T}=\left[t_{0}, T\right]$ fixed, $X_{0}(\omega)$ and $A(t, \omega)$ are independent RVs. Observe that this assumption is realistic from a practical standpoint when dealing with physical models since initial conditions and coefficients involved in the differential equations are not often physically related. Anyway, the subsequent analysis can also be carry out if independence between $X_{0}(\omega)$ and $A(t, \omega)$ is not embraced. As a consequence, if denoting by $\boldsymbol{\xi}_{N}(\omega)=\left(\xi_{1}(\omega), \ldots, \xi_{N}(\omega)\right)$ the random vector whose components are the RVs arising in the KLE of $A(t, \omega)$, then it will be assumed that $X_{0}(\omega)$ and $\boldsymbol{\xi}_{N}(\omega)$, are independent. Additionally, it will be supposed that $X_{0}(\omega)$ is an absolutely continuous $R V$ and $\boldsymbol{\xi}_{N}(\omega)$ is an absolutely continuous random vector whose PDFs will be denoted by $f_{0}\left(x_{0}\right)$ and $f_{\boldsymbol{\xi}_{N}}\left(\xi_{1}, \ldots, \xi_{N}\right)$, respectively. Denoting by $\boldsymbol{\xi}_{N+1}=\left(x_{0}(\omega), \xi_{1}(\omega), \ldots, \xi_{N}(\omega)\right)$, observe that due to independence between $X_{0}(\omega)$ and $\boldsymbol{\xi}_{N}(\omega)$, their joint PDF, $f_{\boldsymbol{\xi}_{N+1}}\left(x_{0}, \xi_{1}, \ldots, \xi_{N}\right)$, is the product of their marginal PDFs, i.e.,

$$
f_{\boldsymbol{\xi}_{N+1}}\left(x_{0}, \xi_{1}, \ldots, \xi_{N}\right)=f_{0}\left(x_{0}\right) f_{\boldsymbol{\xi}_{N}}\left(\xi_{1}, \ldots, \xi_{N}\right)
$$

Summarizing, in the following it will be assumed that 


$$
X_{0}(\omega), \xi_{i}(\omega), 1 \leq i \leq N, \text { are absolutely continuous RVs. }
$$

H2 : $\quad X_{0}(\omega), \boldsymbol{\xi}_{N}(\omega)=\left(\xi_{1}(\omega), \ldots, \xi_{N}(\omega)\right)$ are independent with PDFs $f_{0}\left(x_{0}\right)$ and $f_{\boldsymbol{\xi}_{N}}\left(\xi_{1}, \ldots, \xi_{N}\right)$, respectively.

Moreover, $\mathbb{E}\left[\xi_{i}(\omega)\right]=0$ and $\mathbb{E}\left[\xi_{i}(\omega) \xi_{j}(\omega)\right]=\delta_{i j}$.

Let us assume that $A \equiv A(t, \omega)$ is a continuous time SP such that $A \in$ $\mathrm{L}^{2}\left(\Omega, \mathrm{L}^{2}(\mathcal{T})\right)$ and let $\mu_{A}(t)$ and $c_{A}(s, t)$ denote its mean and covariance functions, respectively. According to Theorem 10.1, the SP $A(t, \omega)$ admits a KLE, and let us consider its truncation of order $N$ (see expression (10.3))

$$
A_{N}(t, \omega)=\mu_{A}(t)+\sum_{j=1}^{N} \sqrt{\nu_{j}} \phi_{j}(t) \xi_{j}(\omega), \quad \omega \in \Omega .
$$

Therefore substituting this expression in (10.4), a formal approximate solution SP to random IVP (10.1) is given by

$$
\begin{aligned}
X_{N}(t, \omega) & =X_{0}(\omega) \operatorname{Exp}\left[\int_{t_{0}}^{t} A_{N}(s, \omega) \mathrm{d} s\right] \\
& =X_{0}(\omega) \operatorname{Exp}\left[\int_{t_{0}}^{t}\left(\mu_{A}(s)+\sum_{j=1}^{N} \sqrt{\nu_{j}} \phi_{j}(s) \xi_{j}(\omega)\right) \mathrm{d} s\right] .
\end{aligned}
$$

Now, RVT technique, stated in Theorem 2.1, will be applied to obtain the 1-PDF of the approximate solution SP (10.6) in terms of the PDFs $f_{0}\left(x_{0}\right)$ and $f_{\boldsymbol{\xi}_{N}}\left(\xi_{1}, \ldots, \xi_{N}\right)$, which are assumed known. As the RVT method applies to RVs, first fix $t \in \mathcal{T}=\left[t_{0}, T\right]$ and then the following mapping $\mathbf{r}: \mathbb{R}^{N+1} \rightarrow \mathbb{R}^{N+1}$ is considered

$$
\begin{gathered}
y_{1}=r_{1}\left(x_{0}, \xi_{1}, \ldots, \xi_{N}\right)=x_{0} \operatorname{Exp}\left[\int_{t_{0}}^{t}\left(\mu_{a}(s)+\sum_{j=1}^{N} \sqrt{\nu_{j}} \phi_{j}(s) \xi_{j}\right) \mathrm{d} s\right], \\
y_{2}=r_{2}\left(x_{0}, \xi_{1}, \ldots, \xi_{N}\right)=\xi_{1}, \\
\vdots \\
y_{N+1}=r_{N+1}\left(x_{0}, \xi_{1}, \ldots, \xi_{N}\right)=\xi_{N},
\end{gathered}
$$

whose inverse transformation $\mathbf{s}=\mathbf{r}^{-1}$ is 


$$
\begin{aligned}
& x_{0}=s_{1}\left(y_{1}, y_{2}, \ldots, y_{N+1}\right)=y_{1} \operatorname{Exp}\left[-\int_{t_{0}}^{t}\left(\mu_{a}(s)+\sum_{j=1}^{N} \sqrt{\nu_{j}} \phi_{j}(s) y_{j+1}\right) \mathrm{d} s\right], \\
& \xi_{1}=s_{2}\left(y_{1}, y_{2}, \ldots, y_{N+1}\right)=y_{2}, \\
& \vdots \\
& \xi_{N}=s_{N+1}\left(y_{1}, y_{2}, \ldots, y_{N+1}\right)=y_{N+1} .
\end{aligned}
$$

The absolute value of the Jacobian of this mapping is given by

$$
\left|J_{N+1}\right|=\operatorname{Exp}\left[-\int_{t_{0}}^{t}\left(\mu_{a}(s)+\sum_{j=1}^{N} \sqrt{\nu_{j}} \phi_{j}(s) y_{j+1}\right) \mathrm{d} s\right] \neq 0
$$

that is non-zero because is defined by an exponential. Then, applying Theorem 2.1 and using independence between $\mathrm{RV} X_{0}$ and random vector $\boldsymbol{\xi}_{N}$, one obtains the joint PDF of random vector $\mathbf{Y}_{N+1}(\omega)=\left(Y_{1}(\omega), Y_{2}(\omega), \ldots, Y_{N+1}(\omega)\right)$ in terms of PDFs $f_{0}\left(x_{0}\right)$ and $f_{\boldsymbol{\xi}_{N}}\left(\xi_{1}, \ldots, \xi_{N}\right)$ (see $(10.5)$ )

$$
\begin{aligned}
& f_{\mathbf{Y}_{N+1}}\left(y_{1}, \ldots, y_{N+1}\right) \\
& =f_{\boldsymbol{\xi}_{N+1}}\left(y_{1} \operatorname{Exp}\left[-\int_{t_{0}}^{t}\left(\mu_{a}(s)+\sum_{j=1}^{N} \sqrt{\nu_{j}} \phi_{j}(s) y_{j+1}\right) \mathrm{d} s\right], y_{2}, \ldots, y_{N+1}\right) \\
& \times \operatorname{Exp}\left[-\int_{t_{0}}^{t}\left(\mu_{a}(s)+\sum_{j=1}^{N} \sqrt{\nu_{j}} \phi_{j}(s) y_{j+1}\right) \mathrm{d} s\right] \\
& =f_{0}\left(y_{1} \operatorname{Exp}\left[-\int_{t_{0}}^{t}\left(\mu_{a}(s)+\sum_{j=1}^{N} \sqrt{\nu_{j}} \phi_{j}(s) y_{j+1}\right) \mathrm{d} s\right]\right) f_{\boldsymbol{\xi}_{N}}\left(y_{2}, \ldots, y_{N+1}\right) \\
& \quad \times \operatorname{Exp}\left[-\int_{t_{0}}^{t}\left(\mu_{a}(s)+\sum_{j=1}^{N} \sqrt{\nu_{j}} \phi_{j}(s) y_{j+1}\right) \mathrm{d} s\right] .
\end{aligned}
$$


Finally, taking $t \in \mathcal{T}=\left[t_{0}, T\right]$ arbitrary and marginalizing expression (10.7) with respect to $Y_{2}=\xi_{1}, \ldots, Y_{N+1}=\xi_{N}$, the 1-PDF of the truncated solution $\mathrm{SP}$ is

$$
\begin{aligned}
& f_{1}^{N}(x, t) \\
& =\int_{\mathbb{R}^{N}} f_{0}\left(x \operatorname{Exp}\left[-\int_{t_{0}}^{t}\left(\mu_{a}(s)+\sum_{j=1}^{N} \sqrt{\nu_{j}} \phi_{j}(s) \xi_{j}\right) \mathrm{d} s\right]\right) f_{\boldsymbol{\xi}_{N}}\left(\xi_{1}, \ldots, \xi_{N}\right) \\
& \quad \times \operatorname{Exp}\left[-\int_{t_{0}}^{t}\left(\mu_{a}(s)+\sum_{j=1}^{N} \sqrt{\nu_{j}} \phi_{j}(s) \xi_{j}\right) \mathrm{d} s\right] \mathrm{d} \xi_{N} \cdots \mathrm{d} \xi_{1} .
\end{aligned}
$$

Observe that the domain of the integral must be understood as the corresponding subset of $\mathbb{R}^{N}$ where the random vector $\boldsymbol{\xi}_{N}(\omega)=\left(\xi_{1}(\omega), \ldots, \xi_{N}(\omega)\right)$ takes values for all $\omega \in \Omega$. As usual in the context of PDFs, this convention will be adopted from now on.

Now, it will be established sufficient conditions in order to guarantee the uniform convergence of this sequence $f_{1}^{N}(x, t)$ to the exact 1-PDF, $f_{1}(x, t)$, i.e.,

$$
\lim _{N \rightarrow+\infty} f_{1}^{N}(x, t)=f_{1}(x, t), \quad \forall(x, t) \in \mathbb{R} \times\left[t_{0}, T\right] .
$$

Since the exact 1-PDF, $f_{1}(x, t)$, is not known, this convergence is established applying the classical Cauchy condition to the sequence $f_{1}^{N}(x, t)$ defined by (10.8). Thus, it will be proved that for $\epsilon>0$ fixed, there exists $n_{0}$ (independent of $(x, t))$, such as

$$
\left|f_{1}^{N}(x, t)-f_{1}^{M}(x, t)\right|<\epsilon, \quad \forall(x, t) \in \mathbb{R} \times\left[t_{0}, T\right], \quad \forall N, M \geq n_{0} .
$$

For the sake of clarity, henceforth the following notation will be used

$$
K_{N}\left(t, \boldsymbol{\xi}_{N}(\omega)\right)=\int_{t_{0}}^{t}\left(\mu_{A}(s)+\sum_{j=1}^{N} \sqrt{\nu_{j}} \phi_{j}(s) \xi_{j}(\omega)\right) \mathrm{d} s
$$


thus expression (10.8) writes

$$
f_{1}^{N}(x, t)=\int_{\mathbb{R}^{N}} f_{0}\left(x \mathrm{e}^{-K_{N}\left(t, \boldsymbol{\xi}_{N}\right)}\right) f_{\boldsymbol{\xi}_{N}}\left(\xi_{1}, \ldots, \xi_{N}\right) \mathrm{e}^{-K_{N}\left(t, \boldsymbol{\xi}_{N}\right)} \mathrm{d} \xi_{N} \cdots \mathrm{d} \xi_{1}
$$

Additionally, the following hypotheses will be assumed throughout in the subsequent development.

$$
\begin{gathered}
f_{0}\left(x_{0}\right) \text { is Lipschitz in } \mathbb{R}, \text { i.e., } \\
\text { H3 : } \exists L_{f_{0}}:\left|f_{0}\left(x_{0,1}\right)-f\left(x_{0,2}\right)\right| \leq L_{f_{0}}\left|x_{0,1}-x_{0,2}\right|, \quad \forall x_{0,1}, x_{0,2} \in \mathbb{R},
\end{gathered}
$$

and

SP $A(t, \omega)$ admits a Karhunen-Loève expansion of type (10.2),

H4 : such that there exists a positive constant $C>0$ such that $\mathbb{E}\left[\mathrm{e}^{-4 K_{N}\left(t, \boldsymbol{\xi}_{N}(\omega)\right)}\right] \leq C$, for all positive integer $N$.

Later it will be showed that this condition can be guaranteed in practice (see Remark 10.2).

Let $\epsilon>0, \mathcal{J} \subset \mathbb{R}$ bounded, $(x, t) \in \mathcal{J} \times\left[t_{0}, T\right]$ an arbitrary point and $N>M$ integers. Taking into account (10.10), below it will be shown that $\left\{f_{1}^{N}(x, t)\right.$ : $N \geq 1\}$ is a Cauchy sequence by using several bounds that will be justified later.

$$
\begin{aligned}
& \left|f_{1}^{N}(x, t)-f_{1}^{M}(x, t)\right| \\
& =\mid \int_{\mathbb{R}^{N}} f_{0}\left(x \mathrm{e}^{-K_{N}\left(t, \boldsymbol{\xi}_{N}\right)}\right) f_{\boldsymbol{\xi}_{N}}\left(\xi_{1}, \ldots, \xi_{N}\right) \mathrm{e}^{-K_{N}\left(t, \boldsymbol{\xi}_{N}\right)} \mathrm{d} \xi_{N} \cdots \mathrm{d} \xi_{1} \\
& \quad-\int_{\mathbb{R}^{M}} f_{0}\left(x \mathrm{e}^{-K_{M}\left(t, \boldsymbol{\xi}_{M}\right)}\right) f_{\boldsymbol{\xi}_{M}}\left(\xi_{1}, \ldots, \xi_{M}\right) \mathrm{e}^{-K_{M}\left(t, \boldsymbol{\xi}_{M}\right)} \mathrm{d} \xi_{M} \cdots \mathrm{d} \xi_{1} \mid \\
& \stackrel{(\mathrm{I})}{=} \mid \int_{\mathbb{R}^{N}}\left[f_{0}\left(x \mathrm{e}^{-K_{N}\left(t, \boldsymbol{\xi}_{N}\right)}\right) \mathrm{e}^{-K_{N}\left(t, \boldsymbol{\xi}_{N}\right)}-f_{0}\left(x \mathrm{e}^{-K_{M}\left(t, \boldsymbol{\xi}_{M}\right)}\right) \mathrm{e}^{-K_{M}\left(t, \boldsymbol{\xi}_{M}\right)}\right] \\
& \quad \times f_{\boldsymbol{\xi}_{N}}\left(\xi_{1}, \ldots, \xi_{N}\right) \mathrm{d} \xi_{N} \cdots \mathrm{d} \xi_{1} \mid
\end{aligned}
$$




$$
\begin{aligned}
& \leq \int_{\mathbb{R}^{N}}\left|\left[f_{0}\left(x \mathrm{e}^{-K_{N}\left(t, \boldsymbol{\xi}_{N}\right)}\right) \mathrm{e}^{-K_{N}\left(t, \boldsymbol{\xi}_{N}\right)}-f_{0}\left(x \mathrm{e}^{-K_{M}\left(t, \boldsymbol{\xi}_{M}\right)}\right) \mathrm{e}^{-K_{M}\left(t, \boldsymbol{\xi}_{M}\right)}\right]\right| \\
& \times f_{\boldsymbol{\xi}_{N}}\left(\xi_{1}, \ldots, \xi_{N}\right) \mathrm{d} \xi_{N} \cdots \mathrm{d} \xi_{1} \\
& =\int_{\mathbb{R}^{N}} \mid\left[f_{0}\left(x \mathrm{e}^{-K_{N}\left(t, \boldsymbol{\xi}_{N}\right)}\right) \mathrm{e}^{-K_{N}\left(t, \boldsymbol{\xi}_{N}\right)}-f_{0}\left(x \mathrm{e}^{-K_{N}\left(t, \boldsymbol{\xi}_{N}\right)}\right) \mathrm{e}^{-K_{M}\left(t, \boldsymbol{\xi}_{M}\right)}\right. \\
& \left.+f_{0}\left(x \mathrm{e}^{-K_{N}\left(t, \boldsymbol{\xi}_{N}\right)}\right) \mathrm{e}^{-K_{M}\left(t, \boldsymbol{\xi}_{M}\right)}-f_{0}\left(x \mathrm{e}^{-K_{M}\left(t, \boldsymbol{\xi}_{M}\right)}\right) \mathrm{e}^{-K_{M}\left(t, \boldsymbol{\xi}_{M}\right)}\right] \mid \\
& \times f_{\boldsymbol{\xi}_{N}}\left(\xi_{1}, \ldots, \xi_{N}\right) \mathrm{d} \xi_{N} \cdots \mathrm{d} \xi_{1} \\
& \leq \int_{\mathbb{R}^{N}}\left[f_{0}\left(x \mathrm{e}^{-K_{N}\left(t, \boldsymbol{\xi}_{N}\right)}\right)\left|\mathrm{e}^{-K_{N}\left(t, \boldsymbol{\xi}_{N}\right)}-\mathrm{e}^{-K_{M}\left(t, \boldsymbol{\xi}_{M}\right)}\right|\right. \\
& \left.+\left|f_{0}\left(x \mathrm{e}^{-K_{N}\left(t, \boldsymbol{\xi}_{N}\right)}\right)-f_{0}\left(x \mathrm{e}^{-K_{M}\left(t, \boldsymbol{\xi}_{M}\right)}\right)\right| \mathrm{e}^{-K_{M}\left(t, \boldsymbol{\xi}_{M}\right)}\right] \\
& \times f_{\boldsymbol{\xi}_{N}}\left(\xi_{1}, \ldots, \xi_{N}\right) \mathrm{d} \xi_{N} \cdots \mathrm{d} \xi_{1} \\
& =\int_{\mathbb{R}^{N}} \underbrace{f_{0}\left(x \mathrm{e}^{-K_{N}\left(t, \boldsymbol{\xi}_{N}\right)}\right)}_{(1)} \underbrace{\left|\mathrm{e}^{-K_{N}\left(t, \boldsymbol{\xi}_{N}\right)}-\mathrm{e}^{-K_{M}\left(t, \boldsymbol{\xi}_{M}\right)}\right|}_{(2)} f_{\boldsymbol{\xi}_{N}}\left(\xi_{1}, \ldots, \xi_{N}\right) \mathrm{d} \xi_{N} \cdots \mathrm{d} \xi_{1} \\
& +\int_{\mathbb{R}^{N}} \underbrace{\left|f_{0}\left(x \mathrm{e}^{-K_{N}\left(t, \boldsymbol{\xi}_{N}\right)}\right)-f_{0}\left(x \mathrm{e}^{-K_{M}\left(t, \boldsymbol{\xi}_{M}\right)}\right)\right|}_{(3)} \mathrm{e}^{-K_{M}\left(t, \boldsymbol{\xi}_{M}\right)} \\
& \times f_{\boldsymbol{\xi}_{N}}\left(\xi_{1}, \ldots, \xi_{N}\right) \mathrm{d} \xi_{N} \cdots \mathrm{d} \xi_{1}
\end{aligned}
$$$$
\stackrel{(\mathrm{II})}{<} L_{f_{0}}|x| \int_{\mathbb{R}^{N}}\left(\mathrm{e}^{-2 K_{N}\left(t, \boldsymbol{\xi}_{N}\right)}+\mathrm{e}^{-K_{N}\left(t, \boldsymbol{\xi}_{N}\right)-K_{M}\left(t, \boldsymbol{\xi}_{M}\right)}\right)\left|K_{N}\left(t, \boldsymbol{\xi}_{N}\right)-K_{M}\left(t, \boldsymbol{\xi}_{M}\right)\right|
$$$$
\times f_{\boldsymbol{\xi}_{N}}\left(\xi_{1}, \ldots, \xi_{N}\right) \mathrm{d} \xi_{N} \cdots \mathrm{d} \xi_{1}
$$$$
+F_{0} \int_{\mathbb{R}^{N}}\left(\mathrm{e}^{-K_{N}\left(t, \boldsymbol{\xi}_{N}\right)}+\mathrm{e}^{-K_{M}\left(t, \boldsymbol{\xi}_{M}\right)}\right)\left|K_{N}\left(t, \boldsymbol{\xi}_{N}\right)-K_{M}\left(t, \boldsymbol{\xi}_{M}\right)\right|
$$$$
\times f_{\boldsymbol{\xi}_{N}}\left(\xi_{1}, \ldots, \xi_{N}\right) \mathrm{d} \xi_{N} \cdots \mathrm{d} \xi_{1}
$$$$
+L_{f_{0}}|x| \int_{\mathbb{R}^{N}}\left(\mathrm{e}^{-2 K_{M}\left(t, \boldsymbol{\xi}_{M}\right)}+\mathrm{e}^{-K_{N}\left(t, \boldsymbol{\xi}_{N}\right)-K_{M}\left(t, \boldsymbol{\xi}_{M}\right)}\right)\left|K_{N}\left(t, \boldsymbol{\xi}_{N}\right)-K_{M}\left(t, \boldsymbol{\xi}_{M}\right)\right|
$$ 


$$
\begin{aligned}
& \times f_{\boldsymbol{\xi}_{N}}\left(\xi_{1}, \ldots, \xi_{N}\right) \mathrm{d} \xi_{N} \cdots \mathrm{d} \xi_{1} \\
= & L_{f_{0}}|x| \mathbb{E}\left[\left(\mathrm{e}^{-2 K_{N}\left(t, \boldsymbol{\xi}_{N}(\omega)\right)}+\mathrm{e}^{-K_{N}\left(t, \boldsymbol{\xi}_{N}(\omega)\right)-K_{M}\left(t, \boldsymbol{\xi}_{M}(\omega)\right)}\right)\right. \\
& \left.\times\left|K_{N}\left(t, \boldsymbol{\xi}_{N}(\omega)\right)-K_{M}\left(t, \boldsymbol{\xi}_{M}(\omega)\right)\right|\right] \\
& +F_{0} \mathbb{E}\left[\left(\mathrm{e}^{-K_{N}\left(t, \boldsymbol{\xi}_{N}(\omega)\right)}+\mathrm{e}^{-K_{M}\left(t, \boldsymbol{\xi}_{M}(\omega)\right)}\right)\right. \\
& \left.\times\left|K_{N}\left(t, \boldsymbol{\xi}_{N}(\omega)\right)-K_{M}\left(t, \boldsymbol{\xi}_{M}(\omega)\right)\right|\right] \\
& +L_{f_{0}}|x| \mathbb{E}\left[\left(\mathrm{e}^{-2 K_{M}\left(t, \boldsymbol{\xi}_{M}(\omega)\right)}+\mathrm{e}^{-K_{N}\left(t, \boldsymbol{\xi}_{N}(\omega)\right)-K_{M}\left(t, \boldsymbol{\xi}_{M}(\omega)\right)}\right)\right. \\
& \left.\times\left|K_{N}\left(t, \boldsymbol{\xi}_{N}(\omega)\right)-K_{M}\left(t, \boldsymbol{\xi}_{M}(\omega)\right)\right|\right] .
\end{aligned}
$$

Now, steps (I)-(II) given in the earlier development will be justified, but for the sake of clarity in the presentation, conclusion is summarized

$$
\begin{aligned}
& \left|f_{1}^{N}(x, t)-f_{1}^{M}(x, t)\right| \\
& \leq L_{f_{0}}|x| \mathbb{E}\left[\left(\mathrm{e}^{-2 K_{N}\left(t, \boldsymbol{\xi}_{N}(\omega)\right)}+\mathrm{e}^{-K_{N}\left(t, \boldsymbol{\xi}_{N}(\omega)\right)-K_{M}\left(t, \boldsymbol{\xi}_{M}(\omega)\right)}\right)\right. \\
& \left.\quad \times\left|K_{N}\left(t, \boldsymbol{\xi}_{N}(\omega)\right)-K_{M}\left(t, \boldsymbol{\xi}_{M}(\omega)\right)\right|\right] \\
& \quad+F_{0} \mathbb{E}\left[\left(\mathrm{e}^{-K_{N}\left(t, \boldsymbol{\xi}_{N}(\omega)\right)}+\mathrm{e}^{-K_{M}\left(t, \boldsymbol{\xi}_{M}(\omega)\right)}\right)\right. \\
& \left.\quad \times\left|K_{N}\left(t, \boldsymbol{\xi}_{N}(\omega)\right)-K_{M}\left(t, \boldsymbol{\xi}_{M}(\omega)\right)\right|\right] \\
& \quad+L_{f_{0}}|x| \mathbb{E}\left[\left(\mathrm{e}^{-2 K_{M}\left(t, \boldsymbol{\xi}_{M}(\omega)\right)}+\mathrm{e}^{-K_{N}\left(t, \boldsymbol{\xi}_{N}(\omega)\right)-K_{M}\left(t, \boldsymbol{\xi}_{M}(\omega)\right)}\right)\right. \\
& \left.\quad \times\left|K_{N}\left(t, \boldsymbol{\xi}_{N}(\omega)\right)-K_{M}\left(t, \boldsymbol{\xi}_{M}(\omega)\right)\right|\right] .
\end{aligned}
$$

Step (I): Let $N>M$, if the joint PDF, $g_{\mathbf{X}_{M}}\left(x_{1}, \ldots, x_{M}\right)$, of a random vector, say,

$$
\mathbf{X}_{N}(\omega)=\left(X_{1}(\omega), \ldots, X_{M}(\omega), X_{M+1}(\omega), \ldots, X_{N}(\omega)\right)
$$


is marginalized with respect to RVs $X_{M+1}(\omega), \ldots, X_{N}(\omega)$, joint PDF of random vector $\mathbf{X}_{M}(\omega)=\left(X_{1}(\omega), \ldots, X_{M}(\omega)\right)$ is obtained, i.e.,

$$
g_{\mathbf{X}_{M}}\left(x_{1}, \ldots, x_{M}\right)=\int_{\mathbb{R}^{N-M}} g_{\mathbf{X}_{N}}\left(x_{1}, \ldots, x_{M}, x_{M+1}, \ldots, x_{N}\right) \mathrm{d} x_{N} \cdots \mathrm{d} x_{M+1} .
$$

Using the notation of previous development with $X_{i} \equiv \xi_{i}, 1 \leq i \leq N$ and

$$
g_{\mathbf{X}_{M}}\left(x_{1}, \ldots, x_{M}\right)=f_{\boldsymbol{\xi}_{M}}\left(\xi_{1}, \ldots, \xi_{M}\right),
$$

(observe that this $g_{\mathbf{X}_{M}}\left(x_{1}, \ldots, x_{M}\right)$ is a PDF), one gets

$$
f_{\boldsymbol{\xi}_{M}}\left(\xi_{1}, \ldots, \xi_{M}\right)=\int_{\mathbb{R}^{N-M}} f_{\boldsymbol{\xi}_{N}}\left(\xi_{1}, \ldots, \xi_{N}\right) \mathrm{d} \xi_{N} \cdots \mathrm{d} \xi_{M+1} .
$$

Therefore, substituting this expression in the left-hand side of (I) this term can be expressed as

$$
\begin{aligned}
& \mid \int_{\mathbb{R}^{N}} f_{0}\left(x \mathrm{e}^{-K_{N}\left(t, \boldsymbol{\xi}_{N}\right)}\right) f_{\boldsymbol{\xi}_{N}}\left(\xi_{1}, \ldots, \xi_{N}\right) \mathrm{e}^{-K_{N}\left(t, \boldsymbol{\xi}_{N}\right)} \mathrm{d} \xi_{N} \cdots \mathrm{d} \xi_{1} \\
& \quad-\int_{\mathbb{R}^{M}} f_{0}\left(x \mathrm{e}^{-K_{M}\left(t, \boldsymbol{\xi}_{M}\right)}\right) f_{\boldsymbol{\xi}_{M}}\left(\xi_{1}, \ldots, \xi_{M}\right) \mathrm{e}^{-K_{M}\left(t, \boldsymbol{\xi}_{M}\right)} \mathrm{d} \xi_{M} \cdots \mathrm{d} \xi_{1} \mid \\
& =\mid \int_{\mathbb{R}^{N}} f_{0}\left(x \mathrm{e}^{-K_{N}\left(t, \boldsymbol{\xi}_{N}\right)}\right) f_{\boldsymbol{\xi}_{N}}\left(\xi_{1}, \ldots, \xi_{N}\right) \mathrm{e}^{-K_{N}\left(t, \boldsymbol{\xi}_{N}\right)} \mathrm{d} \xi_{N} \cdots \mathrm{d} \xi_{1} \\
& \quad-\int_{\mathbb{R}^{M}} f_{0}\left(x \mathrm{e}^{-K_{M}\left(t, \boldsymbol{\xi}_{M}\right)}\right)\left(\int_{\mathbb{R}^{N-M}} f_{\boldsymbol{\xi}_{N}}\left(\xi_{1}, \ldots, \xi_{N}\right) \mathrm{d} \xi_{N} \cdots \mathrm{d} \xi_{M+1}\right) \\
& \quad \times \mathrm{e}^{-K_{M}\left(t, \boldsymbol{\xi}_{M}\right)} \mathrm{d} \xi_{M} \cdots \mathrm{d} \xi_{1} \mid \\
& =\mid \int_{\mathbb{R}^{N}} f_{0}\left(x \mathrm{e}^{-K_{N}\left(t, \boldsymbol{\xi}_{N}\right)}\right) f_{\boldsymbol{\xi}_{N}}\left(\xi_{1}, \ldots, \xi_{N}\right) \mathrm{e}^{-K_{N}\left(t, \boldsymbol{\xi}_{N}\right)} \mathrm{d} \xi_{N} \cdots \mathrm{d} \xi_{1} \\
& \quad-\int_{\mathbb{R}^{N}} f_{0}\left(x \mathrm{e}^{-K_{M}\left(t, \boldsymbol{\xi}_{M}\right)}\right) f_{\boldsymbol{\xi}_{N}}\left(\xi_{1}, \ldots, \xi_{N}\right) \mathrm{e}^{-K_{M}\left(t, \boldsymbol{\xi}_{M}\right)} \mathrm{d} \xi_{N} \cdots \mathrm{d} \xi_{1} \mid
\end{aligned}
$$




$$
\begin{aligned}
= & \mid \int_{\mathbb{R}^{N}}\left[f_{0}\left(x \mathrm{e}^{-K_{N}\left(t, \boldsymbol{\xi}_{N}\right)}\right) f_{\boldsymbol{\xi}_{N}}\left(\xi_{1}, \ldots, \xi_{N}\right) \mathrm{e}^{-K_{N}\left(t, \boldsymbol{\xi}_{N}\right)}-f_{0}\left(x \mathrm{e}^{-K_{M}\left(t, \boldsymbol{\xi}_{M}\right)}\right)\right. \\
& \left.\times f_{\boldsymbol{\xi}_{N}}\left(\xi_{1}, \ldots, \xi_{N}\right) \mathrm{e}^{-K_{M}\left(t, \boldsymbol{\xi}_{M}\right)}\right] \mathrm{d} \xi_{N} \cdots \mathrm{d} \xi_{1} \mid \\
= & \mid \int_{\mathbb{R}^{N}}\left[f_{0}\left(x \mathrm{e}^{-K_{N}\left(t, \boldsymbol{\xi}_{N}\right)}\right) \mathrm{e}^{-K_{N}\left(t, \boldsymbol{\xi}_{N}\right)}-f_{0}\left(x \mathrm{e}^{-K_{M}\left(t, \boldsymbol{\xi}_{M}\right)}\right) \mathrm{e}^{-K_{M}\left(t, \boldsymbol{\xi}_{M}\right)}\right] \\
& \times f_{\boldsymbol{\xi}_{N}}\left(\xi_{1}, \ldots, \xi_{N}\right) \mathrm{d} \xi_{N} \cdots \mathrm{d} \xi_{1} \mid,
\end{aligned}
$$

which is just the right-hand side of (I). This justifies Step (I).

Step (II): Now, bounds used in this step will be legitimated. Without loss of generality, let $F_{0}=f_{0}(0)$ and then first bound the term (1) using hypothesis H3:

$$
\begin{aligned}
f_{0}\left(x \mathrm{e}^{-K_{N}\left(t, \boldsymbol{\xi}_{N}(\omega)\right)}\right) & \leq\left|f_{0}\left(x \mathrm{e}^{-K_{N}\left(t, \boldsymbol{\xi}_{N}(\omega)\right)}\right)-f_{0}(0)\right|+\left|f_{0}(0)\right| \\
& \leq L_{f_{0}}|x| \mathrm{e}^{-K_{N}\left(t, \boldsymbol{\xi}_{N}(\omega)\right)}+F_{0}
\end{aligned}
$$

Secondly, bound for the product of terms (1) and (2) will be obtained as follows

$$
\begin{aligned}
f_{0} & \left(x \mathrm{e}^{-K_{N}\left(t, \boldsymbol{\xi}_{N}\right)}\right)\left|\mathrm{e}^{-K_{N}\left(t, \boldsymbol{\xi}_{N}\right)}-\mathrm{e}^{-K_{M}\left(t, \boldsymbol{\xi}_{M}\right)}\right| \\
\leq & \left(L_{f_{0}}|x| \mathrm{e}^{-K_{N}\left(t, \boldsymbol{\xi}_{N}(\omega)\right)}+F_{0}\right)\left|\mathrm{e}^{-K_{N}\left(t, \boldsymbol{\xi}_{N}\right)}-\mathrm{e}^{-K_{M}\left(t, \boldsymbol{\xi}_{M}\right)}\right| \\
= & L_{f_{0}}|x|\left|\mathrm{e}^{-2 K_{N}\left(t, \boldsymbol{\xi}_{N}\right)}-\mathrm{e}^{-K_{N}\left(t, \boldsymbol{\xi}_{N}\right)-K_{M}\left(t, \boldsymbol{\xi}_{M}\right)}\right| \\
& +F_{0}\left|\mathrm{e}^{-K_{N}\left(t, \boldsymbol{\xi}_{N}\right)}-\mathrm{e}^{-K_{M}\left(t, \boldsymbol{\xi}_{M}\right)}\right| .
\end{aligned}
$$

Now, by applying the Mean Value Theorem twice to function $\mathrm{e}^{-z}$, it is guaranteed that

$$
\left.\exists \delta_{t, \boldsymbol{\xi}_{N}}^{(1)} \in\right] \min \{a, b\}, \max \{a, b\}\left[, \quad \text { where } \left\{\begin{array}{rl}
a & =2 K_{N}\left(t, \boldsymbol{\xi}_{N}\right), \\
b & =K_{N}\left(t, \boldsymbol{\xi}_{N}\right)+K_{M}\left(t, \boldsymbol{\xi}_{M}\right),
\end{array}\right.\right.
$$


such that

$$
\left|\mathrm{e}^{-2 K_{N}\left(t, \boldsymbol{\xi}_{N}\right)}-\mathrm{e}^{-K_{N}\left(t, \boldsymbol{\xi}_{M}\right)-K_{M}\left(t, \boldsymbol{\xi}_{M}\right)}\right|=\mathrm{e}^{-\delta_{t, \boldsymbol{\xi}_{N}}^{(1)}}\left|K_{N}\left(t, \boldsymbol{\xi}_{N}\right)-K_{M}\left(t, \boldsymbol{\xi}_{M}\right)\right|,
$$

and

$$
\left.\exists \delta_{t, \boldsymbol{\xi}_{N}}^{(2)} \in\right] \min \left\{K_{N}\left(t, \boldsymbol{\xi}_{N}\right), K_{M}\left(t, \boldsymbol{\xi}_{M}\right)\right\}, \max \left\{K_{N}\left(t, \boldsymbol{\xi}_{N}\right), K_{M}\left(t, \boldsymbol{\xi}_{M}\right)\right\}[
$$

such that

$$
\left|\mathrm{e}^{-K_{N}\left(t, \boldsymbol{\xi}_{N}\right)}-\mathrm{e}^{-K_{M}\left(t, \boldsymbol{\xi}_{M}\right)}\right|=\mathrm{e}^{-\delta_{t, \boldsymbol{\xi}_{N}}^{(2)}}\left|K_{N}\left(t, \boldsymbol{\xi}_{N}\right)-K_{M}\left(t, \boldsymbol{\xi}_{M}\right)\right|,
$$

respectively. As a consequence of (10.13) and (10.14), one gets

$\mathrm{e}^{-\max \left\{2 K_{N}\left(t, \boldsymbol{\xi}_{N}\right), K_{N}\left(t, \boldsymbol{\xi}_{N}\right)+K_{M}\left(t, \boldsymbol{\xi}_{M}\right)\right\}}<\mathrm{e}^{-\delta_{t, \boldsymbol{\xi}_{N}}^{(1)}}<\mathrm{e}^{-\min \left\{2 K_{N}\left(t, \boldsymbol{\xi}_{N}\right), K_{N}\left(t, \boldsymbol{\xi}_{N}\right)+K_{M}\left(t, \boldsymbol{\xi}_{M}\right)\right\}}$ and

$$
\mathrm{e}^{-\max \left\{K_{N}\left(t, \boldsymbol{\xi}_{N}\right), K_{M}\left(t, \boldsymbol{\xi}_{M}\right)\right\}}<\mathrm{e}^{-\delta_{t, \boldsymbol{\xi}_{N}}^{(2)}}<\mathrm{e}^{-\min \left\{K_{N}\left(t, \boldsymbol{\xi}_{N}\right), K_{M}\left(t, \boldsymbol{\xi}_{M}\right)\right\}},
$$

respectively. Therefore,

$$
\mathrm{e}^{-\delta_{t, \boldsymbol{\xi}_{N}}^{(1)}}<\mathrm{e}^{-2 K_{N}\left(t, \boldsymbol{\xi}_{N}\right)}+\mathrm{e}^{-K_{N}\left(t, \boldsymbol{\xi}_{N}\right)-K_{M}\left(t, \boldsymbol{\xi}_{M}\right)}
$$

and

$$
\mathrm{e}^{-\delta_{t, \boldsymbol{\xi}_{N}}^{(2)}}<\mathrm{e}^{-K_{N}\left(t, \boldsymbol{\xi}_{N}\right)}+\mathrm{e}^{-K_{M}\left(t, \boldsymbol{\xi}_{M}\right)}
$$

respectively. Applying (10.13)-(10.16) in (10.12) one deduces 


$$
\begin{aligned}
& f_{0}\left(x \mathrm{e}^{-K_{N}\left(t, \boldsymbol{\xi}_{N}\right)}\right)\left|\mathrm{e}^{-K_{N}\left(t, \boldsymbol{\xi}_{N}\right)}-\mathrm{e}^{-K_{M}\left(t, \boldsymbol{\xi}_{M}\right)}\right| \\
& \leq L_{f_{0}}|x|\left|\mathrm{e}^{-2 K_{N}\left(t, \boldsymbol{\xi}_{N}\right)}-\mathrm{e}^{-K_{N}\left(t, \boldsymbol{\xi}_{N}\right)-K_{M}\left(t, \boldsymbol{\xi}_{M}\right)}\right| \\
&+F_{0}\left|\mathrm{e}^{-K_{N}\left(t, \boldsymbol{\xi}_{N}\right)}-\mathrm{e}^{-K_{M}\left(t, \boldsymbol{\xi}_{M}\right)}\right| \\
&= L_{f_{0}}|x| \mathrm{e}^{-\delta_{t, \boldsymbol{\xi}_{N}}^{(1)}}\left|K_{N}\left(t, \boldsymbol{\xi}_{N}\right)-K_{M}\left(t, \boldsymbol{\xi}_{M}\right)\right| \\
&+F_{0} \mathrm{e}^{-\delta_{t, \boldsymbol{\xi}_{N}}^{(2)}}\left|K_{N}\left(t, \boldsymbol{\xi}_{N}\right)-K_{M}\left(t, \boldsymbol{\xi}_{M}\right)\right| \\
&<\left\{L_{f_{0}}|x|\left(\mathrm{e}^{-2 K_{N}\left(t, \boldsymbol{\xi}_{N}\right)}+\mathrm{e}^{-K_{N}\left(t, \boldsymbol{\xi}_{N}\right)-K_{M}\left(t, \boldsymbol{\xi}_{M}\right)}\right)\right. \\
&\left.+F_{0}\left(\mathrm{e}^{-K_{N}\left(t, \boldsymbol{\xi}_{N}\right)}+\mathrm{e}^{-K_{M}\left(t, \boldsymbol{\xi}_{M}\right)}\right)\right\}\left|K_{N}\left(t, \boldsymbol{\xi}_{N}\right)-K_{M}\left(t, \boldsymbol{\xi}_{M}\right)\right| .
\end{aligned}
$$

Now, a bound for term (3) will be constructed following a similar argument shown above. Indeed, on the one hand, by applying hypothesis H3 one gets

$$
\left|f_{0}\left(x \mathrm{e}^{-K_{N}\left(t, \boldsymbol{\xi}_{N}\right)}\right)-f_{0}\left(x \mathrm{e}^{-K_{M}\left(t, \boldsymbol{\xi}_{M}\right)}\right)\right| \leq L_{f_{0}}|x|\left|\mathrm{e}^{-K_{N}\left(t, \boldsymbol{\xi}_{N}\right)}-\mathrm{e}^{-K_{M}\left(t, \boldsymbol{\xi}_{M}\right)}\right| .
$$

On the other hand, by applying the Mean Value Theorem to function $\mathrm{e}^{-z}$, it is guaranteed that

$$
\left.\exists \delta_{t, \boldsymbol{\xi}_{N}}^{(3)} \in\right] \min \{a, b\}, \max \{a, b\}\left[, \quad \text { where } \left\{\begin{array}{rl}
a & =2 K_{M}\left(t, \boldsymbol{\xi}_{M}\right), \\
b & =K_{N}\left(t, \boldsymbol{\xi}_{N}\right)+K_{M}\left(t, \boldsymbol{\xi}_{M}\right),
\end{array}\right.\right.
$$

such that

$$
\left|\mathrm{e}^{-K_{N}\left(t, \boldsymbol{\xi}_{N}\right)-K_{M}\left(t, \boldsymbol{\xi}_{M}\right)}-\mathrm{e}^{-2 K_{M}\left(t, \boldsymbol{\xi}_{M}\right)}\right|=\mathrm{e}^{-\delta_{t, \boldsymbol{\xi}_{N}}^{(3)}}\left|K_{N}\left(t, \boldsymbol{\xi}_{N}\right)-K_{M}\left(t, \boldsymbol{\xi}_{M}\right)\right| .
$$

Therefore,

$$
\mathrm{e}^{-\max \left\{2 K_{M}\left(t, \boldsymbol{\xi}_{M}\right), K_{N}\left(t, \boldsymbol{\xi}_{N}\right)+K_{M}\left(t, \boldsymbol{\xi}_{M}\right)\right\}}<\mathrm{e}^{-\delta_{t, \boldsymbol{\xi}_{N}}^{(3)}}<\mathrm{e}^{-\min \left\{2 K_{M}\left(t, \boldsymbol{\xi}_{M}\right), K_{N}\left(t, \boldsymbol{\xi}_{N}\right)+K_{M}\left(t, \boldsymbol{\xi}_{M}\right)\right\}}
$$


and hence

$$
\mathrm{e}^{-\delta_{t, \boldsymbol{\xi}_{N}}^{(3)}}<\mathrm{e}^{-2 K_{M}\left(t, \boldsymbol{\xi}_{M}\right)}+\mathrm{e}^{-K_{N}\left(t, \boldsymbol{\xi}_{N}\right)-K_{M}\left(t, \boldsymbol{\xi}_{M}\right)} .
$$

Multiplying (10.17) by $\mathrm{e}^{-K_{M}\left(t, \boldsymbol{\xi}_{M}\right)}$ and applying (10.18) and (10.19), one deduces

$$
\begin{aligned}
& \left|f_{0}\left(x \mathrm{e}^{-K_{N}\left(t, \boldsymbol{\xi}_{N}\right)}\right)-f_{0}\left(x \mathrm{e}^{-K_{M}\left(t, \boldsymbol{\xi}_{M}\right)}\right)\right| \mathrm{e}^{-K_{M}\left(t, \boldsymbol{\xi}_{M}\right)} \\
& \quad \leq L_{f_{0}}|x|\left|\mathrm{e}^{-K_{N}\left(t, \boldsymbol{\xi}_{N}\right)}-\mathrm{e}^{-K_{M}\left(t, \boldsymbol{\xi}_{M}\right)}\right| \mathrm{e}^{-K_{M}\left(t, \boldsymbol{\xi}_{M}\right)} \\
& \quad=L_{f_{0}}|x|\left|\mathrm{e}^{-K_{N}\left(t, \boldsymbol{\xi}_{N}\right)-K_{M}\left(t, \boldsymbol{\xi}_{M}\right)}-\mathrm{e}^{-2 K_{M}\left(t, \boldsymbol{\xi}_{M}\right)}\right| \\
& \quad=L_{f_{0}}|x| \mathrm{e}^{-\delta_{t, \boldsymbol{\xi}_{N}}^{(3)}}\left|K_{N}\left(t, \boldsymbol{\xi}_{N}\right)-K_{M}\left(t, \boldsymbol{\xi}_{M}\right)\right| \\
& \quad<L_{f_{0}}|x|\left(\mathrm{e}^{-2 K_{M}\left(t, \boldsymbol{\xi}_{M}\right)}+\mathrm{e}^{-K_{N}\left(t, \boldsymbol{\xi}_{N}\right)-K_{M}\left(t, \boldsymbol{\xi}_{M}\right)}\right)\left|K_{N}\left(t, \boldsymbol{\xi}_{N}\right)-K_{M}\left(t, \boldsymbol{\xi}_{M}\right)\right| .
\end{aligned}
$$

Now, a bound for every expectation appearing in (10.11) will be obtained. Applying Cauchy-Schwarz inequality for expectations

$$
\begin{aligned}
\mathbb{E} & {\left[\left(\mathrm{e}^{-2 K_{N}\left(t, \boldsymbol{\xi}_{N}(\omega)\right)}+\mathrm{e}^{-K_{N}\left(t, \boldsymbol{\xi}_{N}(\omega)\right)-K_{M}\left(t, \boldsymbol{\xi}_{M}(\omega)\right)}\right)\left|K_{N}\left(t, \boldsymbol{\xi}_{N}(\omega)\right)-K_{M}\left(t, \boldsymbol{\xi}_{M}(\omega)\right)\right|\right] } \\
= & \mathbb{E}\left[\mathrm{e}^{-2 K_{N}\left(t, \boldsymbol{\xi}_{N}(\omega)\right)}\left|K_{N}\left(t, \boldsymbol{\xi}_{N}(\omega)\right)-K_{M}\left(t, \boldsymbol{\xi}_{M}(\omega)\right)\right|\right] \\
& +\mathbb{E}\left[\mathrm{e}^{-K_{N}\left(t, \boldsymbol{\xi}_{N}(\omega)\right)-K_{M}\left(t, \boldsymbol{\xi}_{M}(\omega)\right)}\left|K_{N}\left(t, \boldsymbol{\xi}_{N}(\omega)\right)-K_{M}\left(t, \boldsymbol{\xi}_{M}(\omega)\right)\right|\right] \\
\leq & \left(\mathbb{E}\left[\mathrm{e}^{-4 K_{N}\left(t, \boldsymbol{\xi}_{N}(\omega)\right)}\right]\right)^{1 / 2}\left(\mathbb{E}\left[\left|K_{N}\left(t, \boldsymbol{\xi}_{N}(\omega)\right)-K_{M}\left(t, \boldsymbol{\xi}_{M}(\omega)\right)\right|^{2}\right]\right)^{1 / 2} \\
& +\left(\mathbb{E}\left[\mathrm{e}^{-2 K_{N}\left(t, \boldsymbol{\xi}_{N}(\omega)\right)} \mathrm{e}^{-2 K_{M}\left(t, \boldsymbol{\xi}_{M}(\omega)\right)}\right]\right)^{1 / 2} \\
& \times\left(\mathbb{E}\left[\left|K_{N}\left(t, \boldsymbol{\xi}_{N}(\omega)\right)-K_{M}\left(t, \boldsymbol{\xi}_{M}(\omega)\right)\right|^{2}\right]\right)^{1 / 2}
\end{aligned}
$$




$$
\begin{aligned}
\leq & \left(\mathbb{E}\left[\mathrm{e}^{-4 K_{N}\left(t, \boldsymbol{\xi}_{N}(\omega)\right)}\right]\right)^{1 / 2}\left(\mathbb{E}\left[\left|K_{N}\left(t, \boldsymbol{\xi}_{N}(\omega)\right)-K_{M}\left(t, \boldsymbol{\xi}_{M}(\omega)\right)\right|^{2}\right]\right)^{1 / 2} \\
& +\left(\mathbb{E}\left[\mathrm{e}^{-4 K_{N}\left(t, \boldsymbol{\xi}_{N}(\omega)\right)}\right]\right)^{1 / 4}\left(\mathbb{E}\left[\mathrm{e}^{-4 K_{M}\left(t, \boldsymbol{\xi}_{M}(\omega)\right)}\right]\right)^{1 / 4} \\
& \times\left(\mathbb{E}\left[\left|K_{N}\left(t, \boldsymbol{\xi}_{N}(\omega)\right)-K_{M}\left(t, \boldsymbol{\xi}_{M}(\omega)\right)\right|^{2}\right]\right)^{1 / 2} \\
= & \left\{\left(\mathbb{E}\left[\mathrm{e}^{-4 K_{N}\left(t, \boldsymbol{\xi}_{N}(\omega)\right)}\right]\right)^{1 / 2}+\left(\mathbb{E}\left[\mathrm{e}^{-4 K_{N}\left(t, \boldsymbol{\xi}_{N}(\omega)\right)}\right]\right)^{1 / 4}\left(\mathbb{E}\left[\mathrm{e}^{-4 K_{M}\left(t, \boldsymbol{\xi}_{M}(\omega)\right)}\right]\right)^{1 / 4}\right\} \\
& \times\left(\mathbb{E}\left[\left|K_{N}\left(t, \boldsymbol{\xi}_{N}(\omega)\right)-K_{M}\left(t, \boldsymbol{\xi}_{M}(\omega)\right)\right|^{2}\right]\right)^{1 / 2},
\end{aligned}
$$

$$
\begin{aligned}
\mathbb{E}[ & \left.\left(\mathrm{e}^{-K_{N}\left(t, \boldsymbol{\xi}_{N}(\omega)\right)}+\mathrm{e}^{-K_{M}\left(t, \boldsymbol{\xi}_{M}(\omega)\right)}\right)\left|K_{N}\left(t, \boldsymbol{\xi}_{N}(\omega)\right)-K_{M}\left(t, \boldsymbol{\xi}_{M}(\omega)\right)\right|\right] \\
= & \mathbb{E}\left[\mathrm{e}^{-K_{N}\left(t, \boldsymbol{\xi}_{N}(\omega)\right)}\left|K_{N}\left(t, \boldsymbol{\xi}_{N}(\omega)\right)-K_{M}\left(t, \boldsymbol{\xi}_{M}(\omega)\right)\right|\right] \\
& +\mathbb{E}\left[\mathrm{e}^{-K_{M}\left(t, \boldsymbol{\xi}_{M}(\omega)\right)}\left|K_{N}\left(t, \boldsymbol{\xi}_{N}(\omega)\right)-K_{M}\left(t, \boldsymbol{\xi}_{M}(\omega)\right)\right|\right] \\
\leq & \left(\mathbb{E}\left[\mathrm{e}^{-2 K_{N}\left(t, \boldsymbol{\xi}_{N}(\omega)\right)}\right]\right)^{1 / 2}\left(\mathbb{E}\left[\left|K_{N}\left(t, \boldsymbol{\xi}_{N}(\omega)\right)-K_{M}\left(t, \boldsymbol{\xi}_{M}(\omega)\right)\right|^{2}\right]\right)^{1 / 2} \\
& +\left(\mathbb{E}\left[\mathrm{e}^{-2 K_{M}\left(t, \boldsymbol{\xi}_{M}(\omega)\right)}\right]\right)^{1 / 2}\left(\mathbb{E}\left[\left|K_{N}\left(t, \boldsymbol{\xi}_{N}(\omega)\right)-K_{M}\left(t, \boldsymbol{\xi}_{M}(\omega)\right)\right|^{2}\right]\right)^{1 / 2} \\
\leq & \left(\mathbb{E}\left[\mathrm{e}^{-4 K_{N}\left(t, \boldsymbol{\xi}_{N}(\omega)\right)}\right]\right)^{1 / 4}\left(\mathbb{E}\left[\left|K_{N}\left(t, \boldsymbol{\xi}_{N}(\omega)\right)-K_{M}\left(t, \boldsymbol{\xi}_{M}(\omega)\right)\right|^{2}\right]\right)^{1 / 2} \\
& +\left(\mathbb{E}\left[\mathrm{e}^{-4 K_{M}\left(t, \boldsymbol{\xi}_{M}(\omega)\right)}\right]\right)^{1 / 4}\left(\mathbb{E}\left[\left|K_{N}\left(t, \boldsymbol{\xi}_{N}(\omega)\right)-K_{M}\left(t, \boldsymbol{\xi}_{M}(\omega)\right)\right|^{2}\right]\right)^{1 / 2} \\
= & \left\{\left(\mathbb{E}\left[\mathrm{e}^{-4 K_{N}\left(t, \boldsymbol{\xi}_{N}(\omega)\right)}\right]\right)^{1 / 4}+\left(\mathbb{E}\left[\mathrm{e}^{-4 K_{M}\left(t, \boldsymbol{\xi}_{M}(\omega)\right)}\right]\right)^{1 / 4}\right\} \\
& \times\left(\mathbb{E}\left[\left|K_{N}\left(t, \boldsymbol{\xi}_{N}(\omega)\right)-K_{M}\left(t, \boldsymbol{\xi}_{M}(\omega)\right)\right|^{2}\right]\right)^{1 / 2},
\end{aligned}
$$


and

$$
\begin{aligned}
\mathbb{E}[ & \left.\left(\mathrm{e}^{-2 K_{M}\left(t, \boldsymbol{\xi}_{M}(\omega)\right)}+\mathrm{e}^{-K_{N}\left(t, \boldsymbol{\xi}_{N}(\omega)\right)-K_{M}\left(t, \boldsymbol{\xi}_{M}(\omega)\right)}\right)\left|K_{N}\left(t, \boldsymbol{\xi}_{N}(\omega)\right)-K_{M}\left(t, \boldsymbol{\xi}_{M}(\omega)\right)\right|\right] \\
= & \mathbb{E}\left[\mathrm{e}^{-2 K_{M}\left(t, \boldsymbol{\xi}_{M}(\omega)\right)}\left|K_{N}\left(t, \boldsymbol{\xi}_{N}(\omega)\right)-K_{M}\left(t, \boldsymbol{\xi}_{M}(\omega)\right)\right|\right] \\
& +\mathbb{E}\left[\mathrm{e}^{-K_{N}\left(t, \boldsymbol{\xi}_{N}(\omega)\right)-K_{M}\left(t, \boldsymbol{\xi}_{M}(\omega)\right)}\left|K_{N}\left(t, \boldsymbol{\xi}_{N}(\omega)\right)-K_{M}\left(t, \boldsymbol{\xi}_{M}(\omega)\right)\right|\right] \\
\leq & \left(\mathbb{E}\left[\mathrm{e}^{-4 K_{M}\left(t, \boldsymbol{\xi}_{M}(\omega)\right)}\right]\right)^{1 / 2}\left(\mathbb{E}\left[\left|K_{N}\left(t, \boldsymbol{\xi}_{N}(\omega)\right)-K_{M}\left(t, \boldsymbol{\xi}_{M}(\omega)\right)\right|^{2}\right]\right)^{1 / 2} \\
& +\left(\mathbb{E}\left[\mathrm{e}^{-2 K_{N}\left(t, \boldsymbol{\xi}_{N}(\omega)\right)} \mathrm{e}^{-2 K_{M}\left(t, \boldsymbol{\xi}_{M}\right)(\omega)}\right]\right)^{1 / 2} \\
& \times\left(\mathbb{E}\left[\left|K_{N}\left(t, \boldsymbol{\xi}_{N}(\omega)\right)-K_{M}\left(t, \boldsymbol{\xi}_{M}(\omega)\right)\right|^{2}\right]\right)^{1 / 2} \\
\leq & \left(\mathbb{E}\left[\mathrm{e}^{-4 K_{M}\left(t, \boldsymbol{\xi}_{M}(\omega)\right)}\right]\right)^{1 / 2}\left(\mathbb{E}\left[\left|K_{N}\left(t, \boldsymbol{\xi}_{N}(\omega)\right)-K_{M}\left(t, \boldsymbol{\xi}_{M}(\omega)\right)\right|^{2}\right]\right)^{1 / 2} \\
& +\left(\mathbb{E}\left[\mathrm{e}^{-4 K_{N}\left(t, \boldsymbol{\xi}_{N}(\omega)\right)}\right]\right)^{1 / 4}\left(\mathbb{E}\left[\mathrm{e}^{-4 K_{M}\left(t, \boldsymbol{\xi}_{M}(\omega)\right)}\right]\right)^{1 / 4} \\
& \times\left(\mathbb{E}\left[\left|K_{N}\left(t, \boldsymbol{\xi}_{N}(\omega)\right)-K_{M}\left(t, \boldsymbol{\xi}_{M}(\omega)\right)\right|^{2}\right]\right)^{1 / 2} \\
\leq & \left\{\left(\mathbb{E}\left[\mathrm{e}^{-4 K_{M}\left(t, \boldsymbol{\xi}_{M}(\omega)\right)}\right]\right)^{1 / 2}+\left(\mathbb{E}\left[\mathrm{e}^{-4 K_{N}\left(t, \boldsymbol{\xi}_{N}(\omega)\right)}\right]\right)^{1 / 4}\left(\mathbb{E}\left[\mathrm{e}^{-4 K_{M}\left(t, \boldsymbol{\xi}_{M}(\omega)\right)}\right]\right)^{1 / 4}\right\} \\
& \times\left(\mathbb{E}\left[\left|K_{N}\left(t, \boldsymbol{\xi}_{N}(\omega)\right)-K_{M}\left(t, \boldsymbol{\xi}_{M}(\omega)\right)\right|^{2}\right]\right)^{1 / 2} \cdot \\
& \\
& \\
& \\
& \\
& \\
&
\end{aligned}
$$

By substituting the three bounds given in (10.20)-(10.22) into expression (10.11) 


$$
\begin{aligned}
& \left|f_{1}^{N}(x, t)-f_{1}^{M}(x, t)\right| \\
& \leq \quad\left[L _ { f _ { 0 } } | x | \left\{\left(\mathbb{E}\left[\mathrm{e}^{-4 K_{N}\left(t, \boldsymbol{\xi}_{N}(\omega)\right)}\right]\right)^{1 / 2}+\left(\mathbb{E}\left[\mathrm{e}^{-4 K_{N}\left(t, \boldsymbol{\xi}_{N}(\omega)\right)}\right]\right)^{1 / 4}\right.\right. \\
& \left.\quad \times\left(\mathbb{E}\left[\mathrm{e}^{-4 K_{M}\left(t, \boldsymbol{\xi}_{M}(\omega)\right)}\right]\right)^{1 / 4}\right\} \\
& \quad+F_{0}\left\{\left(\mathbb{E}\left[\mathrm{e}^{-4 K_{N}\left(t, \boldsymbol{\xi}_{N}(\omega)\right)}\right]\right)^{1 / 4}+\left(\mathbb{E}\left[\mathrm{e}^{-4 K_{M}\left(t, \boldsymbol{\xi}_{M}(\omega)\right)}\right]\right)^{1 / 4}\right\} \\
& \quad+L_{f_{0}}|x|\left\{\left(\mathbb{E}\left[\mathrm{e}^{-4 K_{M}\left(t, \boldsymbol{\xi}_{M}(\omega)\right)}\right]\right)^{1 / 2}+\left(\mathbb{E}\left[\mathrm{e}^{-4 K_{N}\left(t, \boldsymbol{\xi}_{N}(\omega)\right)}\right]\right)^{1 / 4}\right. \\
& \left.\left.\quad \times\left(\mathbb{E}\left[\mathrm{e}^{-4 K_{M}\left(t, \boldsymbol{\xi}_{M}(\omega)\right)}\right]\right)^{1 / 4}\right\}\right]\left(\mathbb{E}\left[\left|K_{N}\left(t, \boldsymbol{\xi}_{N}(\omega)\right)-K_{M}\left(t, \boldsymbol{\xi}_{M}(\omega)\right)\right|^{2}\right]\right)^{1 / 2} .
\end{aligned}
$$

Now, using hypothesis H4 one gets

$$
\begin{aligned}
\left|f_{1}^{N}(x, t)-f_{1}^{M}(x, t)\right| & \\
\leq & {\left[L_{f_{0}}|x|\left\{C^{1 / 2}+C^{1 / 4} \times C^{1 / 4}\right\}+F_{0}\left\{C^{1 / 4}+C^{1 / 4}\right\}\right.} \\
& \left.+L_{f_{0}}|x|\left\{C^{1 / 2}+C^{1 / 4} \times C^{1 / 4}\right\}\right]\left(\mathbb{E}\left[\left|K_{N}\left(t, \boldsymbol{\xi}_{N}(\omega)\right)-K_{M}\left(t, \boldsymbol{\xi}_{M}(\omega)\right)\right|^{2}\right]\right)^{1 / 2} \\
= & {[\alpha|x|+\beta]\left(\mathbb{E}\left[\left|K_{N}\left(t, \boldsymbol{\xi}_{N}(\omega)\right)-K_{M}\left(t, \boldsymbol{\xi}_{M}(\omega)\right)\right|^{2}\right]\right)^{1 / 2}, }
\end{aligned}
$$

where

$$
\alpha=4 C^{1 / 2} L_{f_{0}}, \quad \beta=2 C^{1 / 4} F_{0} .
$$

Let us observe by applying the Cauchy-Schwarz inequality for integrals and the definition of the norm $\|\cdot\|_{L^{2}\left(\Omega, L^{2}(\mathcal{T})\right)}$ for $\mathcal{T}=\left[t_{0}, T\right]$, that 


$$
\begin{aligned}
\mathbb{E}\left[\left|K_{N}\left(t, \boldsymbol{\xi}_{N}(\omega)\right)-K_{M}\left(t, \boldsymbol{\xi}_{M}(\omega)\right)\right|^{2}\right] \\
=\mathbb{E}\left[\left(\int_{t_{0}}^{t} \sum_{j=M+1}^{N} \sqrt{\nu_{j}} \phi_{j}(s) \xi_{j}(\omega) \mathrm{d} s\right)^{2}\right] \\
\leq \mathbb{E}\left[\left(\int_{t_{0}}^{t} 1^{2} \mathrm{~d} s\right)\left(\int_{t_{0}}^{t}\left(\sum_{j=M+1}^{N} \sqrt{\nu_{j}} \phi_{j}(s) \xi_{j}(\omega)\right)^{2} \mathrm{~d} s\right)\right] \\
=\left(t-t_{0}\right) \mathbb{E}\left[\int_{t_{0}}^{t}\left(\sum_{j=M+1}^{N} \sqrt{\nu_{j}} \phi_{j}(s) \xi_{j}(\omega)\right)^{2} \mathrm{~d} s\right] \\
\quad \leq\left(T-t_{0}\right) \mathbb{E}\left[\int_{t_{0}}^{T}\left(\sum_{j=M+1}^{N} \sqrt{\nu_{j}} \phi_{j}(s) \xi_{j}(\omega)\right)^{2} \mathrm{~d} s\right] \\
=\left(T-t_{0}\right) \mathbb{E}\left[\int_{t_{0}}^{T}\left(A_{N}(t, \omega)-A_{M}(t, \omega)\right)^{2} \mathrm{~d} s\right] \\
=\left(T-t_{0}\right) \int_{t_{0}}^{T} \mathbb{E}\left[\left(A_{N}(t, \omega)-A_{M}(t, \omega)\right)^{2}\right] \mathrm{d} s \\
=\left(T-t_{0}\right)\left(\left\|A_{N}-A_{M}\right\|_{\mathrm{L}^{2}\left(\Omega, \mathrm{L}^{2}\left(\left[t_{0}, T\right]\right)\right)}\right)^{2} .
\end{aligned}
$$

Substituting this latter conclusion in (10.23) and applying the Cauchy convergence condition for the KLE of diffusion coefficient $A(t, \omega)$ in the norm $\|\cdot\|_{L^{2}\left(\Omega, L^{2}\left(\left[t_{0}, T\right]\right)\right)}($ see Theorem 10.1), one deduces

$$
\begin{aligned}
\left|f_{1}^{N}(x, t)-f_{1}^{M}(x, t)\right| & \leq[\alpha|x|+\beta]\left(\mathbb{E}\left[\left|K_{N}\left(t, \boldsymbol{\xi}_{N}(\omega)\right)-K_{M}\left(t, \boldsymbol{\xi}_{M}(\omega)\right)\right|^{2}\right]\right)^{1 / 2} \\
& \leq[\alpha|x|+\beta] \sqrt{T-t_{0}}\left\|A_{N}-A_{M}\right\|_{\mathrm{L}^{2}\left(\Omega, \mathrm{L}^{2}\left(\left[t_{0}, T\right]\right)\right)} \underset{N, M \rightarrow+\infty}{\longrightarrow} 0 .
\end{aligned}
$$

This proves that $\left\{f_{1}^{N} \equiv f_{1}^{N}(x, t): N \geq 1\right\}$ is a uniformly Cauchy sequence in $\mathcal{J} \times\left[t_{0}, T\right]$ for all $\mathcal{J} \subset \mathbb{R}$ bounded. 
Summarizing, the following result has been established.

Proposition 10.1 Under hypotheses H1-H4, the sequence $\left\{f_{1}^{N}(x, t): N \geq 1\right\}$ of 1-PDFs, defined by (10.8), converges uniformly in $(x, t) \in \mathcal{J} \times \mathcal{T}$ for all $\mathcal{J} \subset \mathbb{R}$ bounded, to the exact $1-P D F, f_{1}(x, t)$, of solution SP of random IVP (10.1).

Remark 10.1 Here, it will be shown that hypothesis H3 is fulfilled by the PDF of a wide variety of RVs. In fact, as a consequence of the Mean Value Theorem it is well known that if a function, say $f_{0}$, has bounded first derivative in in $\mathbb{R}$, then $f_{0}$ is Lipschitz over the whole real line. It is straightforwardly to check that the PDF of important RVs such as Uniform, Beta, Gaussian, Gamma, etc. have bounded first derivative over the whole real line. For example, if $f_{0}$ is the PDF of an Exponential RV of parameter $\lambda>0$, then $f_{0}\left(x_{0}\right)=\lambda \mathrm{e}^{-\lambda x_{0}}$, $x_{0}, \lambda>0$. As $\frac{\mathrm{d} f_{0}}{\mathrm{~d} x_{0}}=-\lambda^{2} \mathrm{e}^{-\lambda x_{0}}$ is continuous in $\left[0,+\infty\left[\right.\right.$ and $\lim _{x_{0} \rightarrow+\infty} \frac{\mathrm{d} f_{0}}{\mathrm{~d} x_{0}}=0$, therefore $f_{0}\left(x_{0}\right)$ is Lipschitz in $[0,+\infty[$.

Remark 10.2 It will be shown that hypothesis $\mathrm{H}_{4}$ is not restrictive in practice. In fact, it is important to observe that given the coefficient $A(t, \omega) \in$ $\mathrm{L}^{2}\left(\Omega, \mathrm{L}^{2}(\mathcal{T})\right)$ of random IVP $(10.1)$, then according to KLE, the involved $R V s$ $\xi_{j}(\omega)$ can be chosen in many ways so that $\mathbb{E}\left[\xi_{j}(\omega)\right]=0, \mathbb{V}\left[\xi_{j}(\omega)\right]=1$ and they are uncorrelated $\left(\mathbb{E}\left[\xi_{i}(\omega) \xi_{j}(\omega)\right]=0\right.$ for $\left.i \neq j\right)$. As in our case they must be absolutely continuous RVs, they can be chosen, for example, as uncorrelated Gaussian RVs with zero mean and unit variance, $\xi_{j}(\omega) \sim N(0 ; 1)$. Next, it will be proven that making this choice, then hypothesis $\mathrm{H}_{4}$ holds. First, observe that taking into account (10.9), the expectation involved in $\mathrm{H}_{4}$ can be written as

$$
\begin{aligned}
\mathbb{E}\left[\mathrm{e}^{b K_{M}\left(t, \boldsymbol{\xi}_{M}(\omega)\right)}\right] & =\mathrm{e}^{b \int_{t_{0}}^{t} \mu_{A}(s) \mathrm{d} s} \mathbb{E}\left[\prod_{j=1}^{M} \mathrm{e}^{b \boldsymbol{\xi}_{j}(\omega) \sqrt{\nu_{j}} \int_{t_{0}}^{t} \phi_{j}(s) \mathrm{d} s}\right] \\
& =\mathrm{e}^{b \int_{t_{0}}^{t} \mu_{A}(s) \mathrm{d} s} \prod_{j=1}^{M} \mathbb{E}\left[\mathrm{e}^{b \boldsymbol{\xi}_{j}(\omega) \sqrt{\nu_{j}} \int_{t_{0}}^{t} \phi_{j}(s) \mathrm{d} s}\right],
\end{aligned}
$$

where in the last step it is used that $\xi_{j}(\omega)$ are independent $R V s$ (since they are uncorrelated and Gaussian), hence the expectation of the product is the product of expectations. Now, the following property 


$$
\mathbb{E}\left[\mathrm{e}^{\lambda Z(\omega)}\right]=\mathrm{e}^{\frac{\lambda^{2}}{2}}, \quad \lambda \in \mathbb{R}, Z(\omega) \sim N(0 ; 1),
$$

is used to compute every factor of the last product. This leads to

$$
\begin{aligned}
\mathbb{E}\left[\mathrm{e}^{b K_{M}\left(t, \boldsymbol{\xi}_{M}(\omega)\right)}\right] & =\mathrm{e}^{b \int_{t_{0}}^{t} \mu_{A}(s) \mathrm{d} s} \prod_{j=1}^{M} \mathrm{e}^{\frac{b^{2}}{2} \nu_{j}\left(\int_{t_{0}}^{t} \phi_{j}(s) \mathrm{d} s\right)^{2}} \\
& =\mathrm{e}^{b \int_{t_{0}}^{t} \mu_{A}(s) \mathrm{d} s} \mathrm{e}^{\frac{b^{2}}{2} \sum_{j=1}^{M} \nu_{j}\left(\int_{t_{0}}^{t} \phi_{j}(s) \mathrm{d} s\right)^{2}} .
\end{aligned}
$$

Applying the Cauchy-Schwarz inequality for integrals one gets

$$
\begin{aligned}
\left(\int_{t_{0}}^{t} \phi_{j}(s) \mathrm{d} s\right)^{2} & \leq\left(t-t_{0}\right)\left(\int_{t_{0}}^{t}\left(\phi_{j}(s)\right)^{2} \mathrm{~d} s\right) \\
& \leq\left(T-t_{0}\right)\left(\int_{t_{0}}^{T}\left(\phi_{j}(s)\right)^{2} \mathrm{~d} s\right), \quad t_{0} \leq t \leq T .
\end{aligned}
$$

Therefore, expression (10.24) can be bounded as follows

$$
\begin{aligned}
\mathbb{E}\left[\mathrm{e}^{b K_{M}\left(t, \boldsymbol{\xi}_{M}(\omega)\right)}\right] & \leq \mathrm{e}^{b \int_{t_{0}}^{t} \mu_{A}(s) \mathrm{d} s} \mathrm{e}^{\frac{b^{2}}{2}\left(T-t_{0}\right) \sum_{j=1}^{M} \nu_{j} \int_{t_{0}}^{T}\left(\phi_{j}(s)\right)^{2} \mathrm{~d} s} \\
& \leq \mathrm{e}^{b \int_{t_{0}}^{t} \mu_{A}(s) \mathrm{d} s} \mathrm{e}^{\frac{b^{2}}{2}\left(T-t_{0}\right) \int_{t_{0}}^{T}\left(\sum_{j=1}^{\infty} \nu_{j}\left(\phi_{j}(s)\right)^{2}\right) \mathrm{d} s} .
\end{aligned}
$$

Now, let us bound every integral term in the right-hand side of this expression, thus proving the finiteness of $\mathbb{E}\left[\mathrm{e}^{b K_{M}\left(t, \boldsymbol{\xi}_{M}(\omega)\right)}\right]<+\infty$. As a consequence, taking $b=4$ and important scenario where hypothesis $H_{4}$ holds will be shown. On the one hand, by Cauchy-Schwarz inequality for integrals, applying that $\left(\mu_{A}(s)\right)^{2}=$ $\left(\mathbb{E}[(A(s)])^{2} \leq \mathbb{E}\left[(A(s))^{2}\right]\right.$ and the fact that $A \in \mathrm{L}^{2}\left(\Omega, \mathrm{L}^{2}\left(\left[t_{0}, T\right]\right)\right)$, one gets 


$$
\begin{aligned}
\int_{t_{0}}^{t}\left|\mu_{A}(s)\right| \mathrm{d} s & \leq \int_{t_{0}}^{T}\left|\mu_{A}(s)\right| \mathrm{d} s \\
& \leq \sqrt{T-t_{0}}\left(\int_{t_{0}}^{t}\left(\mu_{A}(s)\right)^{2} \mathrm{~d} s\right)^{1 / 2} \\
& \leq \sqrt{T-t_{0}}\left(\int_{t_{0}}^{T} \mathbb{E}\left[(A(s))^{2}\right] \mathrm{d} s\right)^{1 / 2} \\
& =\sqrt{T-t_{0}}\|A\|_{\mathrm{L}^{2}\left(\Omega, \mathrm{L}^{2}\left(\left[t_{0}, T\right]\right)\right)}<+\infty
\end{aligned}
$$

Since $\left|\int_{t_{0}}^{t} \mu_{A}(s) \mathrm{d} s\right| \leq \int_{t_{0}}^{t}\left|\mu_{A}(s)\right| \mathrm{d} s$, this justifies $\mathrm{e}^{b \int_{t_{0}}^{t} \mu_{A}(s) \mathrm{d} s}<+\infty$, which is the first factor of the right-hand side in (10.25). On the other hand, let us observe

$$
\begin{aligned}
& \mathbb{E}\left[(A(s))^{2}\right]=\left(\mu_{A}(s)\right)^{2}+\sum_{j=1}^{\infty} \nu_{j}\left(\phi_{j}(s)\right)^{2}, \\
& A(s)=\mu_{A}(s)+\sum_{j=1}^{+\infty} \sqrt{\nu_{j}} \phi_{j}(s) \xi_{j}(\omega) \text { in } \mathrm{L}^{2}\left(\Omega, \mathrm{L}^{2}\left(\left[t_{0}, T\right]\right)\right),
\end{aligned}
$$

hence

$$
\sum_{j=1}^{\infty} \nu_{j}\left(\phi_{j}(s)\right)^{2} \leq \mathbb{E}\left[(A(s))^{2}\right]
$$

and

$$
\int_{t_{0}}^{T} \sum_{j=1}^{\infty} \nu_{j}\left(\phi_{j}(s)\right)^{2} \mathrm{~d} s \leq \int_{t_{0}}^{T} \mathbb{E}\left[(A(s))^{2}\right] \mathrm{d} s=\left(\|A\|_{\mathrm{L}^{2}\left(\Omega, \mathrm{L}^{2}\left(\left[t_{0}, T\right]\right)\right)}\right)^{2}<+\infty
$$

Therefore, the second factor of the right-hand side in (10.25) is finite, i.e. $\mathrm{e}^{\frac{b^{2}}{2}\left(T-t_{0}\right) \int_{t_{0}}^{T}\left(\sum_{j=1}^{\infty} \nu_{j}\left(\phi_{j}(s)\right)^{2}\right) \mathrm{d} s}<+\infty$. Summarizing, if $\xi_{j}(\omega)$ in the KLE are chosen as uncorrelated standard Gaussian RVs, then hypothesis $\mathrm{H}_{4}$ is guaranteed. 


\subsection{Numerical examples}

In this section, two examples will be shown. In the first example, the SP $A(t, \omega)$, playing the role of diffusion coefficient in random IVP $(10.1)$, is the socalled Brownian motion or standard Wiener process. As the exact distribution of the Brownian motion is known, then the exact 1-PDF $f_{1}(x, t)$ of solution SP $X(t, \omega)$ to $(10.1)$ can be derived. Hence, this first example will be used as a test to compare the approximations, $f_{1}^{N}(x, t)$ given by (10.8), for different values of the truncation order $N$ against the exact values. In the second example a covariance function is considered. Then, from the knowledge of its eigenpairs $\left\{\left(\nu_{j}, \phi_{j}(t)\right): j \geq 1\right\}$, approximations of the 1-PDF are given. In both examples, approximations of the mean and standard deviation of $X(t, \omega)$ are given from $f_{1}^{N}(x, t)$. Finally, in both examples error measures are provided in order to quantify the accuracy of approximations of the 1-PDF, the mean and the standard deviation.

\subsubsection{Example 1: Brownian motion}

In this example, $\mathrm{SP} A(t, \omega) \equiv B(t, \omega)$ is the Brownian motion or standard Wiener and $t_{0}=0$. Then, it is known that $\mu_{A}(t)=0$ and $\mathbb{V}[A(t, \omega)]=1$, $\forall t \in \mathcal{T}=[0, T], T>0$. In addition, the covariance function is given by

$$
c_{A}(s, t)=\min (s, t), \quad(s, t) \in \mathcal{T} \times \mathcal{T},
$$

which has the following eigenvalues and normalized eigenfunctions

$$
\nu_{j}=\frac{4 T^{2}}{\pi^{2}(2 j-1)^{2}}, \quad \phi_{j}(t)=\sqrt{\frac{2}{T}} \sin \left(\frac{(2 j-1) \pi t}{2 T}\right), \quad j=1,2, \ldots
$$

Then, the 1-PDF of the truncated solution SP $X_{N}(t, \omega)$ is obtained substituting (10.26) in (10.8)

$$
f_{1}^{N}(x, t)=\int_{\mathbb{R}^{N}} f_{0}\left(x \prod_{j=1}^{N} \mathrm{e}^{-h_{j}(t) \xi_{j}}\right) f_{\boldsymbol{\xi}_{N}}\left(\xi_{1}, \ldots, \xi_{N}\right) \prod_{j=1}^{N} \mathrm{e}^{-h_{j}(t) \xi_{j}} \mathrm{~d} \xi_{N} \cdots \mathrm{d} \xi_{1},
$$


where

$$
h_{j}(t)=\left(\frac{2 T}{(2 j-1) \pi)}\right)^{2} \sqrt{\frac{2}{T}}\left(1-\cos \left(\frac{(2 j-1) \pi t}{2 T}\right)\right) .
$$

In this example the 1-PDF of the exact solution SP, $X(t, \omega)$, can be computed taking into account that $\hat{B}(t)=\int_{0}^{t} B(s) d s \sim N\left(0 ; \sqrt{\frac{t^{3}}{3}}\right)$. Hence $\hat{B}(t) \stackrel{\mathrm{d}}{=}$ $\sqrt{\frac{t^{3}}{3}} Z, Z \sim \mathrm{N}(0 ; 1)$, that is, the SP $\hat{B}(t)$ has the same distribution as $\operatorname{RV} \sqrt{\frac{t^{3}}{3}} Z$, being $Z$ a standard Gaussian RV. Using RVT method, it is straightforwardly to check that the $1-\mathrm{PDF}$ of $X(t, \omega)$ is given by

$$
f_{1}(x, t)=\int_{-\infty}^{\infty} f_{0}\left(x \mathrm{e}^{-\sqrt{\frac{t^{3}}{3}} z}\right) f_{Z}(z) \mathrm{e}^{-\sqrt{\frac{t^{3}}{3}} z} \mathrm{~d} z
$$

where $f_{0}\left(x_{0}\right)$ and $f_{Z}(z)$ denote the PDFs of RVs $X_{0}$ and $Z$, respectively.

In Figure 10.1, it is shown 3D-plots of the exact 1-PDF (left) and two approximations $f_{1}^{N}(x, t)$ using $(10.27)-(10.28)$ with $N=1$ (center) and $N=2$ (right), respectively, over the time interval $[0, T]=[0,2]$. it have been taken $\xi_{j}(\omega), j \in\{1,2\}$ uncorrelated standard Gaussian RVs and $X_{0}(\omega)$ a Uniform $\mathrm{RV}$ on the interval $[0,1]$, i.e., $X_{0}(\omega) \sim \operatorname{Un}([0,1])$. As in the theoretical development, $X_{0}(\omega)$ is assumed to be independent of $\xi_{1}(\omega)$ and $\xi_{2}(\omega)$. Notice that this assumption has been already used in (10.29). In the context of this example, clearly all hypotheses H1-H4 hold (see Remarks 10.1 and 10.2 to check H3 and H4, respectively). From Figure 10.1, one can see that the first and second order truncations (plots in the center and in the right, respectively) are close to the 1-PDF of the exact solution (plot in the left). This feature can be observed in detail in Figure 10.2 where the exact PDF, $f_{1}(x, t)$, and the two previous approximations, $f_{1}^{1}(x, t)$ and $f_{1}^{2}(x, t)$ have been plotted in different time instants $(t \in\{0.1,1,2\})$. For sake of clarity, in Table 10.1, total error is collected, defined by the following expression (10.30), between the exact 1-PDF and the approximation with order of truncation $N$ at different times instants

$$
e_{N}^{\mathrm{PDF}}(t)=\int_{-\infty}^{\infty}\left|f_{1}(x, t)-f_{1}^{N}(x, t)\right| \mathrm{d} x
$$

Finally, in Figure 10.3 exact mean and exact standard deviation are compared with the approximations. From these plots, one can see that approximations 

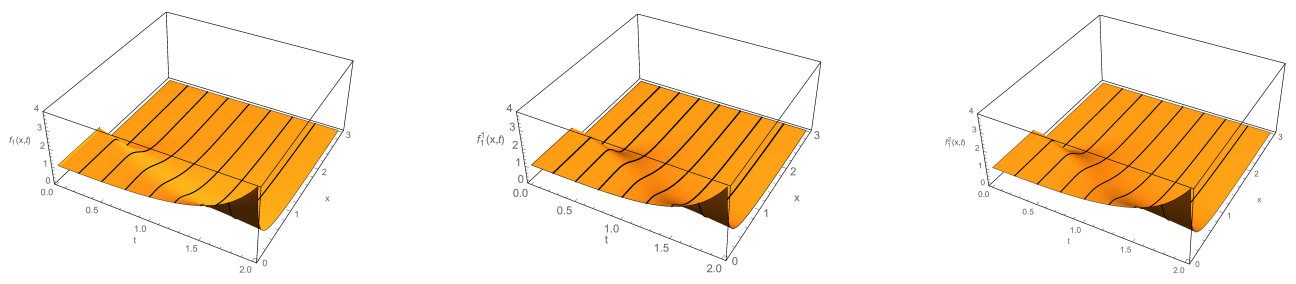

Figure 10.1: Left: 1-PDF of the exact solution SP given by (10.29). Center: 1-PDF of the first truncation given by (10.27)-(10.28) with $N=1$. Right: $1-\mathrm{PDF}$ of the second truncation given by (10.27)-(10.28) with $N=2$.

\begin{tabular}{ccc}
\hline$e_{N}^{\mathrm{PDF}}(t)$ & $N=1$ & $N=2$ \\
\hline \hline$t=0.1$ & 0.010021 & 0.008682 \\
\hline$t=1$ & 0.077919 & 0.008663 \\
\hline$t=2$ & 0.005310 & 0.000832 \\
\hline
\end{tabular}

Table 10.1: Error measure $e_{N}^{\mathrm{PDF}}(t)$ defined by (10.30) for different time instants, $t \in\{0.1$, $1,2\}$, and truncation orders $N \in\{1,2\}$, in the context of Example 10.4.1.

are good, being slower the convergence of standard deviation. The errors of these approximations are shown in Table 10.2. These figures have been calculated using

$$
e_{N}^{\mu}=\int_{t_{0}}^{T}\left|\mu_{X}(t)-\mu_{X}^{N}(t)\right| \mathrm{d} t, \quad e_{N}^{\sigma}=\int_{t_{0}}^{T}\left|\sigma_{X}(t)-\sigma_{X}^{N}(t)\right| \mathrm{d} t,
$$

with $t_{0}=0$ and $T=2$.

\begin{tabular}{ccccc}
\hline Error & $N=1$ & $N=2$ & $N=3$ & $N=4$ \\
\hline \hline Mean $e_{N}^{\mu}$ & 0.055567 & 0.005541 & 0.002425 & 0.000871 \\
\hline Standard deviation $e_{N}^{\sigma}$ & 0.383975 & 0.169942 & 0.159339 & 0.151808 \\
\hline
\end{tabular}

Table 10.2: Values of errors $e_{N}^{\mu}$ and $e_{N}^{\sigma}$ for the mean and standard deviation, given by (10.31) using different orders of truncation $N$, in the context of Example 10.4.1. 

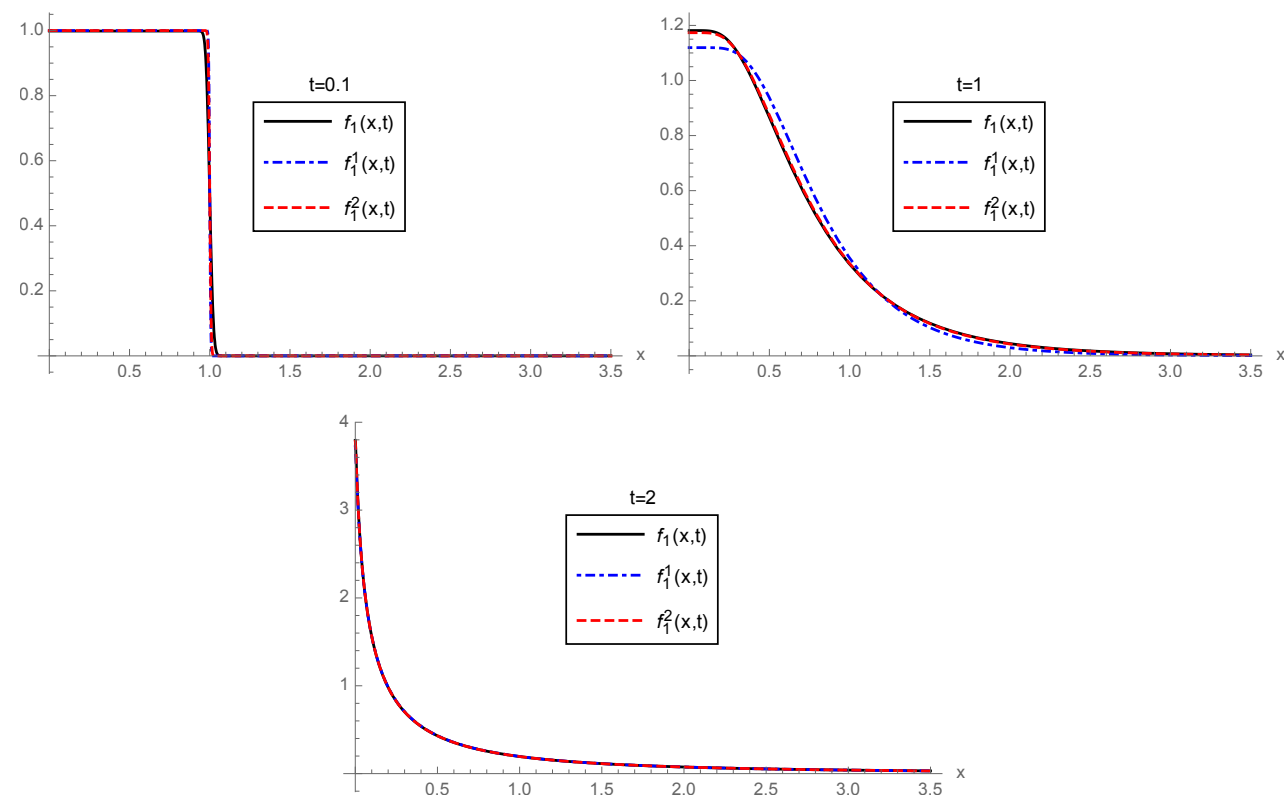

Figure 10.2: 1-PDF $f_{1}(x, t)$ of the exact solution SP and the two first truncations $f_{1}^{N}(x, t)$, $N \in\{1,2\}$, for different values of $t$ in the context of Example 10.4.1. Top left: $t=0.1$. Top right: $t=1$. Bottom: $t=2$.

\subsubsection{Example 2: Exponential covariance}

As the KLE relies upon the covariance function $c_{A}(s, t)$ of the SP, in this example the covariance function of the SP $A(t, \omega)$ will be assumed known instead of giving the SP itself. Let us consider the following covariance function, usually referred to as exponential covariance,

$$
c_{A}(s, t)=\mathrm{e}^{-\frac{|s-t|}{b}}, \quad(s, t) \in \mathcal{T} \times \mathcal{T}, \quad \mathcal{T}=[-a, a], a>0
$$

where $b>0$ is often termed the correlation length, since it reflects the rate at which the correlation decays between two times of the process. The eigenvalues and normalized eigenfunctions of the covariance function are given by $[61, \mathrm{p}$. 294-295] 

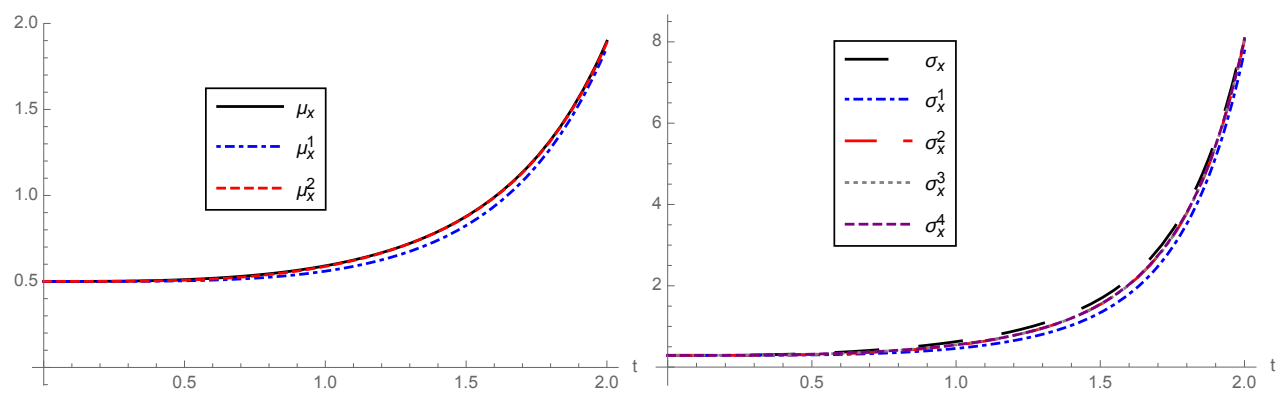

Figure 10.3: In the context of Example 10.4.1. Left: Exact mean $\left(\mu_{x}\right)$ of solution and its approximations using truncations of order $N \in\{1,2\}$ ( $\mu_{x}^{1}$ and $\mu_{x}^{2}$, respectively). Right: Exact standard deviation $\left(\sigma_{x}\right)$ of solution and its approximations using truncations of order $N \in\{1,2,3,4\}\left(\sigma_{x}^{i}, i \in\{1,2,3,4\}\right)$.

$$
\begin{aligned}
& \phi_{j}(t)=\frac{\cos \left(z_{j} x\right)}{\sqrt{a+\frac{\sin \left(2 z_{j} a\right)}{2 z_{j}}}}, \quad \nu_{j}=\frac{2 c}{z_{j}^{2}+c^{2}}, \quad j \text { odd }, \\
& \phi_{j}^{*}(t)=\frac{\sin \left(z_{j}^{*} x\right)}{\sqrt{a-\frac{\sin \left(2 z_{j}^{*} a\right)}{2 z_{j}^{*}}}}, \quad \nu_{j}^{*}=\frac{2 c}{\left(z_{j}^{*}\right)^{2}+c^{2}}, \quad j \text { even, }
\end{aligned}
$$

being $c=1 / b$ and $z_{j}, z_{j}^{*}$ solutions of the following transcendental equations

$$
\begin{aligned}
& c-z_{j} \tan \left(z_{j} a\right)=0, \quad j \text { odd } \\
& z_{j}^{*}+c \tan \left(z_{j}^{*} a\right)=0, \quad j \text { even. }
\end{aligned}
$$

Then, considering the mean of the SP $A(t, \omega)$ is zero, the KLE of $A(t, \omega)$ is given by

$$
A(t, \omega)=\sum_{j=1}^{\infty}\left(\sqrt{\nu_{2 j-1}} \phi_{2 j-1}(t) \xi_{2 j-1}(\omega)+\sqrt{\nu_{2 j}^{*}} \phi_{2 j}^{*}(t) \xi_{2 j}^{*}(\omega)\right)
$$

Next, it will be shown approximations, $f_{1}^{N}(x, t)$, of the 1-PDF, $f_{1}(x, t)$, of solution SP, $X(t, \omega)$, to random IVP $(10.1)$, being $A(t, \omega)$ represented by the 
KLE (10.33). These approximations will be constructed using the expression (10.8) with different orders of truncation $N$.

In Figure 10.4, $f_{1}^{N}(x, t)$ has been plotted with $N=1$ and $N=2$ for two different correlation lengths, $b=0.1$ and $b=1$, over the time domain $\mathcal{T}=$ $[-0.5,0.5]$ corresponding to $a=0.5$. Therefore, initial time instant is $t_{0}=$ -0.5. $\xi_{j}(\omega), j \in\{1,2\}$, have been chosen as uncorrelated standard Gaussian RVs to represent the SP $A(t, \omega)$ by KLE (10.33). Initial condition $X_{0}(\omega)$ is assumed to be an Exponential RV with mean 1/4, i.e., $X_{0}(\omega) \sim \operatorname{Exp}(4)$. Hence, by Remark 10.1, hypothesis H3 holds. $X_{0}(\omega), \xi_{1}(\omega)$ and $\xi_{2}(\omega)$ are assumed independent RVs. From Figure 10.4, one can observe that both $f_{1}^{1}(x, t)$ and $f_{1}^{2}(x, t)$ are very similar, then indicating quick convergence with respect to the truncation order $N$. This happens for both values of parameter $b$ over the whole space-time domain. For the sake of clarity, in Figure 10.5 both approximations are shown in the middle point $t=0$ of the domain $\mathcal{T}$ for $b=0.1$ and $b=1$. As an indicator of convergence, in Table 10.3 the following error has been computed

$$
\hat{e}_{N}^{\mathrm{PDF}}(t)=\int_{-\infty}^{\infty}\left|f_{1}^{N}(x, t)-f_{1}^{N-1}(x, t)\right| \mathrm{d} x
$$

between consecutive approximations over the whole spacial domain at $t=0$ (middle time instant) for both values of parameter $b$.

\begin{tabular}{ccc}
\hline$\hat{e}_{N}^{\mathrm{PDF}}(0)$ & $N=2$ & $N=3$ \\
\hline \hline$b=1$ & 0.0106515 & 0.0000164 \\
\hline$b=0.1$ & 0.0147553 & 0.0008538
\end{tabular}

Table 10.3: Error measure $\hat{e}_{N}(t)$ defined by (10.34) for time instant $t=0$, and truncation orders, $N \in\{2,3\}$ for $b=1$ and $b=0.1$, in the context of Example 10.4.2.

In Figure 10.6, mean and standard deviation are shown with $b=0.1$ and $b=1$ for different orders of truncation.

To account for the error, in Table 10.4 and Table 10.5 the following errors for $b=0.1$ and $b=1$ respectively, with $t_{0}=-0.5$ and $T=0.5$ are computed

$$
\hat{e}_{N}^{\mu}=\int_{t_{0}}^{T}\left|\mu_{X}^{N}(t)-\mu_{X}^{N-1}(t)\right| \mathrm{d} t, \quad \hat{e}_{N}^{\sigma}=\int_{t_{0}}^{T}\left|\sigma_{X}^{N}(t)-\sigma_{X}^{N-1}(t)\right| \mathrm{d} t
$$



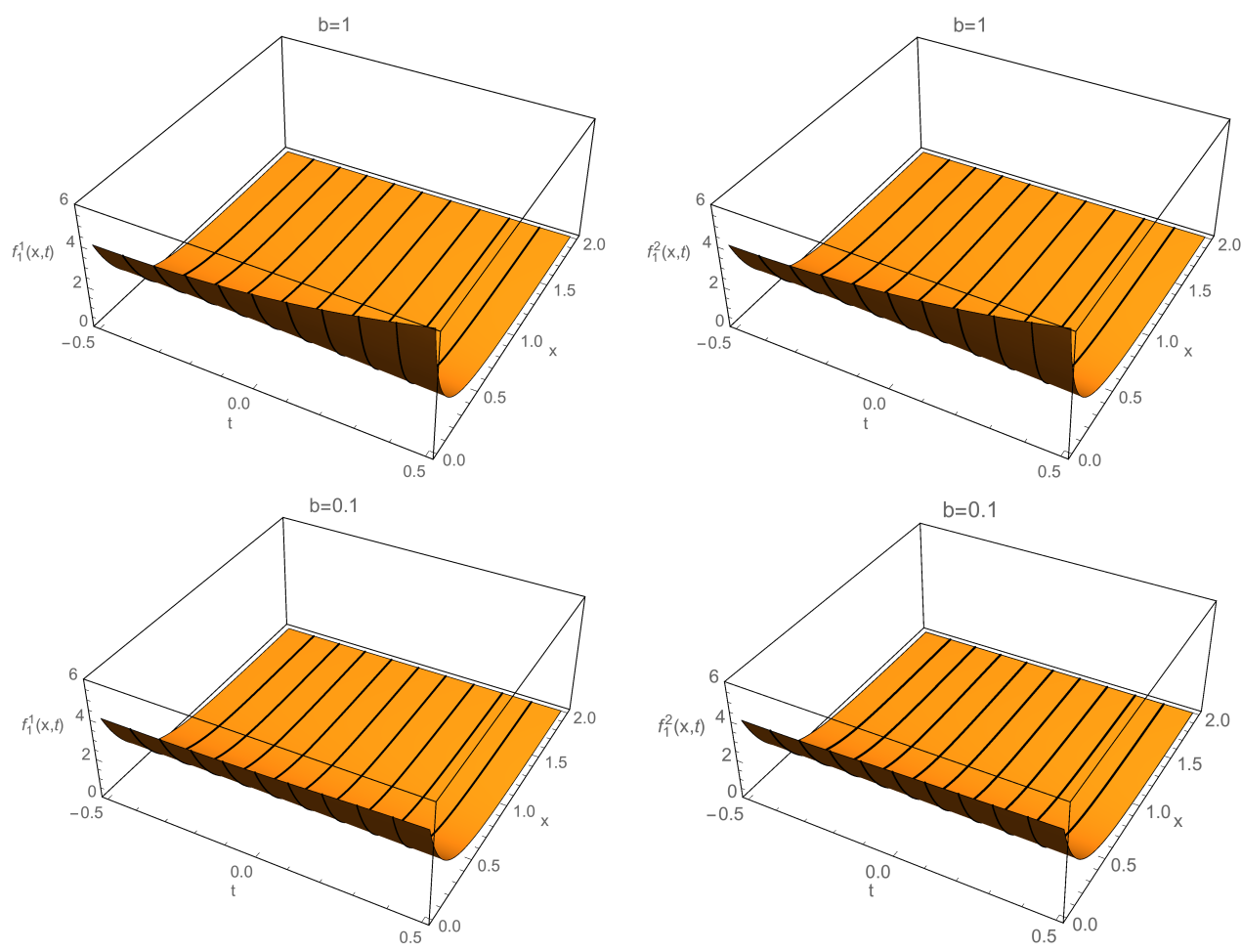

Figure 10.4: In the context of Example 10.4.2. Top: 1-PDF of the truncations $N=1$ and $N=2$, with $b=1$ Bottom: 1 -PDF of the truncations $N=1$ and $N=2$, with $b=0.1$.

where $\mu_{X}^{n}(t)$ and $\sigma_{X}^{n}(t), n=N-1, N$, are approximations to the mean and the standard deviation.

From Table 10.3, one observes that the error $\hat{e}_{N}^{\mathrm{PDF}}(0)$ is smaller for $b=1$ than $b=0.1$. This same behaviour happens regarding the approximations of the mean and the standard deviation, namely, they are better for $b=1$ than for $b=0.1$, except for $N=2$ (see Table 10.4 and Table 10.5). This result can be expected from the decay of eigenvalues $v_{j}$. In fact, it is well-known that $[61$, p. 204]

$$
\left\|A(t, \omega)-A_{N}(t, \omega)\right\|_{\mathrm{L}^{2}\left(\Omega, \mathrm{L}^{2}(\mathcal{T})\right)}=\int_{\mathcal{T}} \mathbb{V}[A(t, \omega)] \mathrm{d} t-\sum_{j=1}^{N} \nu_{j} .
$$



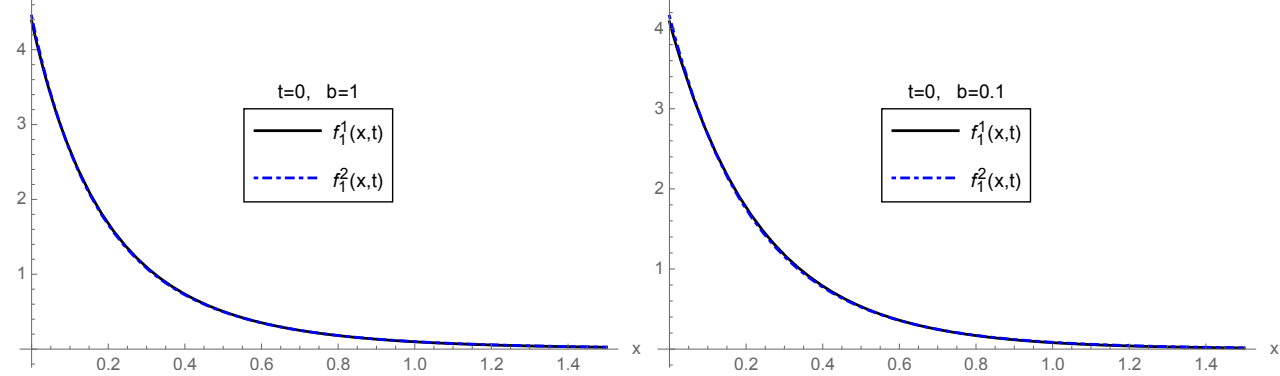

Figure 10.5: 1-PDF of the truncations $N \in\{1,2\}$, for $t=0$, in the context of Example 10.4.2. Left: $b=1$. Right: $b=0.1$.

\begin{tabular}{ccccc}
\hline error $(b=0.1)$ & $N=2$ & $N=3$ & $N=4$ & $N=5$ \\
\hline \hline$\hat{e}_{N}^{\mu}$ & 0.0018717 & 0.0004470 & 0.0001983 & 0.0000631 \\
\hline$\hat{e}_{N}^{\sigma}$ & 0.0057412 & 0.0013707 & 0.0006206 & 0.0003282 \\
\hline
\end{tabular}

Table 10.4: Errors $\hat{e}_{N}^{\mu}$ and $\hat{e}_{N}^{\sigma}$ for the mean and the standard deviation, defined by (10.35) respectively, using different orders of truncations $(N \in\{2,3,4,5\})$ and correlation length $b=0.1$, in the context of Example 10.4.2.

Then, the first $N$ eigenvalues $\nu_{j}$ can be added to determine the truncation parameter $N$ for a given error tolerance, say $\epsilon>0$, when approximating diffusion coefficient $A(t, \omega)$ using the KLE. Obviously, the greater the values of $v_{j}$, the smaller the value of $N$. In the context of this example the decay of eigenvalues $\nu_{j}$ depends on the choice of parameter $b$. The bigger $b$ the faster decay of $\nu_{j}$ and, as a consequence, a smaller value of the truncation parameter $N$ will be required to achieve the accuracy $\epsilon$ in order to approximate $A(t, \omega)$. In Table 10.6, the first eigenvalues of the covariance function (10.32) are shown for $b=0.1$ and $b=1$ over the time interval $\mathcal{T}=[-0.5,0.5]$. These eigenvalues have been represented in Figure 10.7. As it can be observed in this plot, the eigenvalues corresponding to $b=1$ decay faster than those ones corresponding to $b=0.1$. This fact is in agreement with figures collected in Table 10.3 (corresponding to approximation of 1-PDF) and in Tables 10.4 and 10.5 (corresponding to approximations of mean and standard deviation), where error associated to $b=1$ is smaller than to $b=0.1$, for a fixed truncation order $N$. 

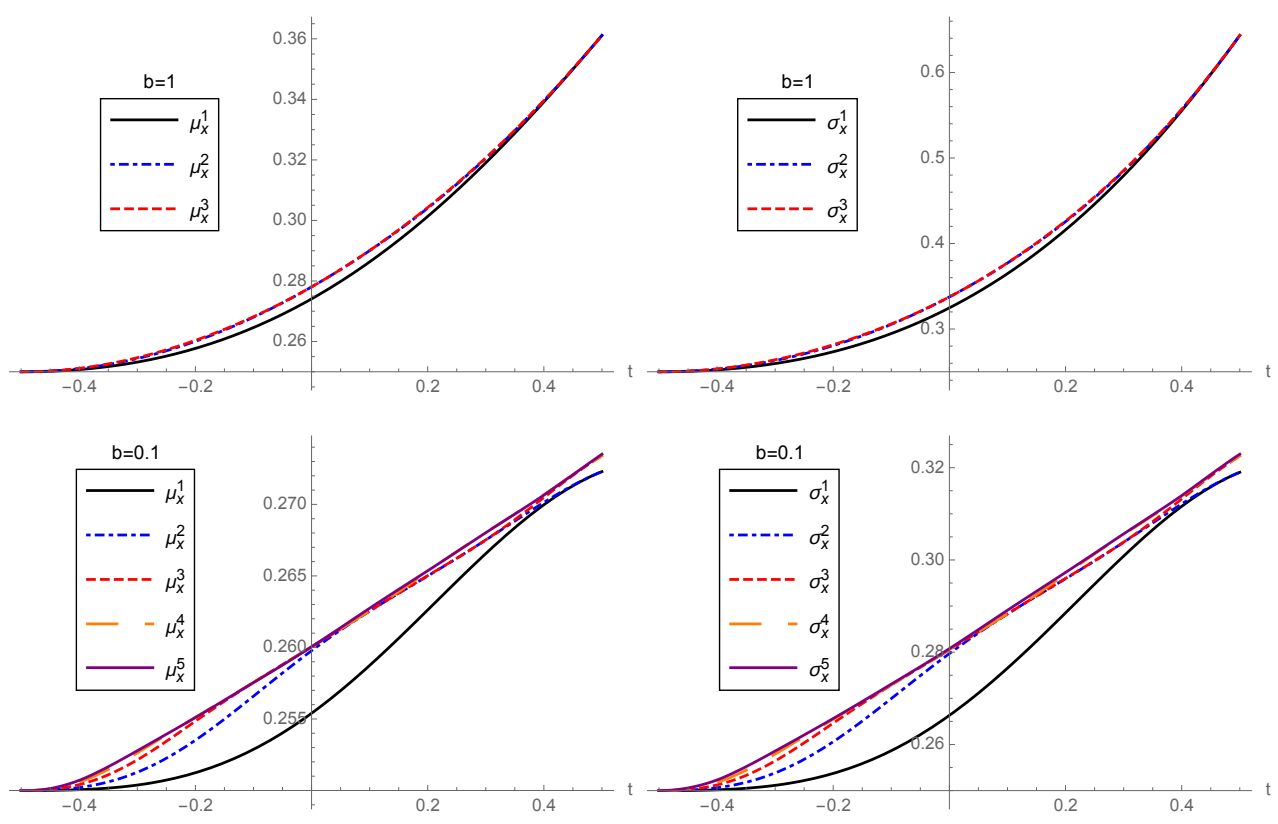

Figure 10.6: In the context of Example 10.4.2. Top: Approximations of the mean and standard deviation using different orders of truncation $N \in\{1,2,3\}$ with correlation length parameter $b=1$. Bottom: Approximations of the mean and standard deviation using different orders of truncation $N \in\{1,2,3,4,5\}$ with correlation length parameter $b=0.1$.

In the context of this example, where neither the exact 1-PDF nor the exact mean and standard deviation are not available, it is very interesting to establish a stopping criterion in order to determine the order of truncation $N$ for a given error tolerance $\epsilon>0$. Just as an example to illustrate the way findings can be used in this regard, if $\epsilon=10^{-3}$ then, according to Table 10.4 and Table 10.5 , it is enough to take $N=3$ as the order of truncation for both values of parameter $b$, while $N=3$ and $N=4$ are required to guarantee the same accuracy for the standard deviation when $b=1$ and $b=0.1$, respectively. 


\begin{tabular}{ccc}
\hline error $(b=1)$ & $N=2$ & $N=3$ \\
\hline \hline$\hat{e}_{N}^{\mu}$ & 0.0019514 & 0.0001370 \\
\hline \hline$\hat{e}_{N}^{\sigma}$ & 0.0064182 & 0.0004473 \\
\hline
\end{tabular}

Table 10.5: Errors $\hat{e}_{N}^{\mu}$ and $\hat{e}_{N}^{\sigma}$ for the mean and the standard deviation, defined by (10.35) respectively, using different orders of truncations $(N \in\{2,3,4,5\})$ and correlation length $b=1$, in the context of Example 10.4.2.

\begin{tabular}{cccccc}
\hline & $\nu_{1}$ & $\nu_{2}$ & $\nu_{3}$ & $\nu_{4}$ & $\nu_{5}$ \\
\hline \hline$b=0.1$ & 0.187083 & 0.156046 & 0.121154 & 0.091324 & 0.068736 \\
\hline$b=1$ & 0.738813 & 0.138000 & 0.045089 & 0.021329 & 0.012279 \\
\hline \hline & $\nu_{6}$ & $\nu_{7}$ & $\nu_{8}$ & $\nu_{9}$ & $\nu_{10}$ \\
\hline \hline$b=0.1$ & 0.052403 & 0.040695 & 0.032225 & 0.025998 & 0.021333 \\
\hline$b=1$ & 0.007945 & 0.005551 & 0.004093 & 0.003142 & 0.002486 \\
\hline
\end{tabular}

Table 10.6: First eigenvalues, $\nu_{j}$, of the covariance function (10.32) for $j \in\{1,2, \ldots, 10\}$ for $b=0.1$ and $b=1$ over the time interval $\mathcal{T}=[-0.5,0.5]$, in the context of Example 10.4.2.

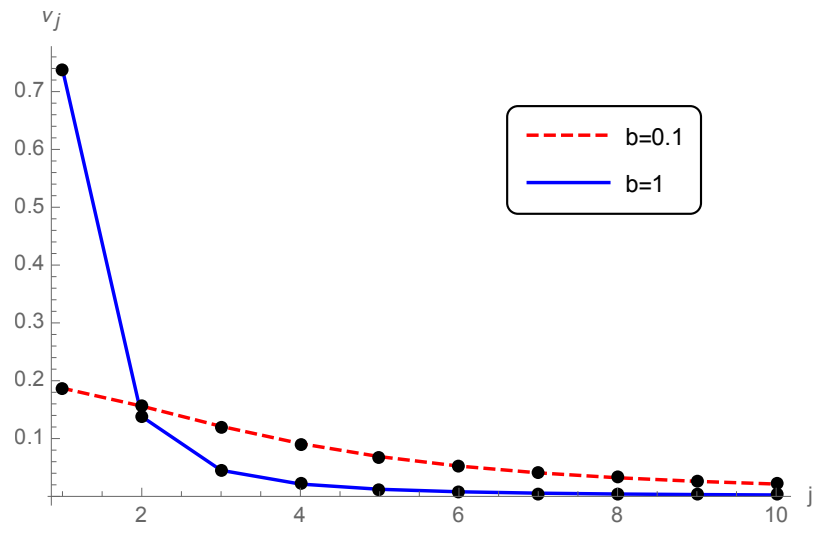

Figure 10.7: First eigenvalues, $\nu_{j}$, of the covariance function (10.32), in the context of Example 10.4.2. 


\subsection{Conclusions}

In this chapter, it have been constructed approximations of the first probability density function to the linear homogeneous first-order random differential equation. It has been proved that these approximations are convergent under mild conditions upon initial condition and diffusion coefficient which are assumed to be an initial condition and a stochastic process, respectively. The key idea to construct these approximations has been to combine the Random Variable Transformation technique and the Karhunen-Loève expansion. Two illustrative examples have been considered showing that approximations converge very rapidly. Indeed, just a few terms are need to approximate the first probability density function in both examples. The first probability density function has been used to approximate both the mean and the variance in both examples. All numerical results are also satisfactory. Finally, as it has been underlined in the motivation of the chapter, although the formulation of the target problem appears to be simple, the analysis does not. This is a genuine feature usually met when deterministic results are extended to the random scenario. 


\section{Chapter 11}

\section{Conclusions}

The main objective of this dissertation has been to solve, from a probabilistic point of view, several fundamental random initial value problems. The mathematical nature of these problems is based upon random difference and differential equations. In both cases, randomness has been considered in all input parameters (coefficients, source term and initial conditions). For the sake of generality, in most of scenarios treated, input random parameters have been considered via absolutely continuous random variables without assuming statistical independence and having arbitrary joint probability density functions, that include both standard and non-standard distributions. Unlike most contributions dealing with random difference and differential equations focus on solving the corresponding equations and then they compute the mean and the variance, in this dissertation one addresses an ambitious target, namely, the computation of the first probability density function of the solution stochastic process. In this manner, the solution is probabilistically characterized at every time instant, allowing for the computation of every one-dimensional statistical moment (mean, variance, skewness, kurtosis, etc.) as well as the determination of probabilities that the solution lies on a set of particular interest.

The cornerstone tool to conduct the stochastic analysis throughout this dissertation has been the Random Variable Transformation technique. This method turns out very useful to determine the probability density function of a random vector that results from a mapping of another random vector whose probabi- 
lity density function is known. This technique has been adequately applied to the framework of both random difference and differential equations to account for uncertainty, via the first probability density function. An important feature of this dissertation is that all theoretical findings have been illustrated through numerical experiments and, when it has been possible, modelling relevant problems using real data. It is worthy to point out that specific techniques to estimate random model parameters, in the context of random difference and differential equations, have been required to deal with mathematical models throughout this dissertation.

As it is detailed below, the application of Random Variable Transformation technique has been made in two different contexts, first when an exact representation to the solution stochastic process is available, and secondly in the case that an approximate representation, for example via series expansion, is known.

Chapters 3-8 deal with random initial value problems where the exact solution is known. Specifically, Chapters $3-5$ are devoted to study the following random differential equations:

- Homogeneous Riccati differential equation, Chapter 3.

- Non-homogeneous Riccati differential equation to model SIS-type epidemiological model, Chapter 4.

- Bernoulli differential equation, Chapter 5.

On the other hand, the theory has been extended to both random linear systems of difference and differential equations. This is presented in Chapter 6 . Chapter 7 and 8 are devoted to extend important results belonging to classical Markov chains, by means of the complete randomization (initial condition and transition matrix) of these models. Furthermore, the theoretical results obtained for randomized Markov chains have been applied to model the diffusion of a technology and to study the stroke disease using real data retrieved from official sources.

So far, all previous chapters have been devoted to study random difference and differential equations where uncertainty is just considered via random variables, while Chapters 9 and 10 address random differential equations whose randomness is considered via stochastic processes.

Chapter 9 focusses on second-order linear differential equations whose coefficients are analytic stochastic processes about a regular point. Specifically, 
one computes approximations of the first probability density function of the solution stochastic process taking advantage of a random power series representation together with the application of Random Variable Transformation technique. The theoretical analysis is completed by means of several numerical experiments.

Chapter 10 addresses the computation of reliable approximations of the first probability density function of the solution of random first-order linear differential equations, where the diffusion coefficient has a denumerable degree of randomness. Then, it is approximated via a truncated Karhunen-Loève expansion and, the Random Variable Transformation technique is applied to obtain approximations of the first probability density function.

In these two closing chapters, one demonstrates the convergence of approximations to the exact first probability density function, under mild conditions.

The main conclusion that can be drawn in this dissertation is the capability of the Random Variable Transformation method, combined with other techniques (Poincaré expansion, Karhunen-Loève expansion, linearization, etc.) to determine, exact or approximately, the first probability density function of the solution of random difference and differential equations. Furthermore, it has been illustrated that the computation of such density is very useful in mathematical modelling of real problems. 



\section{Bibliography}

[1] M. Abramowitz and I.A. Stegan. Handbook of Mathematical Functions, with Formulas, Graphs, and Mathematical Tables. New York: Dover, 1972, p. 14. ISBN: 0486612724 (cit. on p. 121).

[2] E. Allen. Modeling with Itô Stochastic Differential Equations. Springer, 2007. ISBN: 9781402059537 (cit. on pp. 2, 72).

[3] F. Antoñanzas et al. "Cost-Effectiveness of the combination therapy of dutasteride and Tamsulosin in the treatment of benign Prostatic Hyperlasia in Spain". In: Actas Urol. Esp. 35.2 (2011), pp. 65-71. DOI: $10.1016 / \mathrm{j}$. acuro.2010.11.008 (cit. on p. 146).

[4] U. M. Ascher, R. M. Mattheij, and R. D. Rusell. Numerical Solution of Boundary Value Problems for Ordinary Differential Equations. New Jersey: Englewood Cliffs: Prentice Hall, 1988. ISBN: 9780898713541 (cit. on p. 28).

[5] R. C. Aster, B. Borchers, and C. H. Thurber. Parameter Estimation and Inverse Problems. Oxford: Academic Press, 2013. ISBN: 9780123850485 (cit. on p. 84).

[6] X. Badia et al. "Análisis de la relación coste-efectividad a corto y largo plazo de clopidogrel añadido a terapia estándar en pacientes con síndrome 
coronario agudo en España". In: Rev. Esp. Cardiol. 58 (2005), pp. 13851395. DOI: 1016/S0300-8932(05)74068-9 (cit. on p. 146).

[7] E. Behrends. Introduction to Markov Chains: With Special Emphasis on Rapid Mixing. Advanced Lectures in Mathematics. Vieweg+Teubner Verlag, 1999. ISBN: 9783528069865 (cit. on p. 111).

[8] L. von Bertalanffy. "A quantitative theory of organic growth (Inquiries on Growth Laws II)". In: Hum. Biol. 10 (1938), pp. 181-213 (cit. on p. 82).

[9] L. von Bertalanffy. "Quantitative laws in metabolism and growth". In: Q. Rev. Biol. 32.3 (1957), pp. 217-231 (cit. on p. 82).

[10] K. A. Bjorndal, L.B. Bolten, and M.Y. Chaloupka. "Green turtle somatic growth model: evidence fordensity dependece". In: Ecol. Appl. 10.1 (2000), pp. 269-282. DOI: 10.1890/1051-0761 (2000)010 [0269 : GTSGME] 2.0.CO;2 (cit. on p. 82).

[11] F. Brauer and C. Castillo-Chávez. Mathematical Models in Population Biology and Epidemiology. New York: Springer, 2001. ISBN: 9781461416852 (cit. on pp. 28, 54).

[12] A. Briggs and M. Sculpher. "An Introduction to Markov Modelling for Economic Evaluation". In: PharmacoEconomics 13.4 (1998), pp. 397-409. DOI: $10.2165 / 00019053-199813040-00003$ (cit. on p. 134).

[13] G. Calbo, J. C. Cortés, and L. Jódar. "Random Hermite differential equations: Mean square power series solutions and statistical properties". In: Appl. Math. Comput. 218.7 (2011), pp. 3654-3666. DOI: $10.1016 /$ j .amc . 2011.09.008 (cit. on pp. 3, 161).

[14] M. Carletti. "Numerical solution of stochastic differential problems in the biosciences". In: J. Comput. Appl. Math. 185.2 (2006), pp. 422-440. DoI: 10.1016/j.cam.2005.03.020 (cit. on p. 2).

[15] M. C. Casabán et al. "A comprehensive probabilistic solution of random SIS-type epidemiological models using the Random Variable Transformation technique". In: Commun. Nonlinear. Sci. Numer. Simul. 32 (2016), pp. 199-210. DOI: 10.1016/j.cnsns.2014.12.016 (cit. on p. 137). 
[16] M. C. Casabán et al. "Determining the first probability density function of linear random initial value problems by the Random Variable Transformation (RVT) technique: A comprehensive study". In: Abstract Appl. Anal. ID248512 (2014), pp. 1-25. DOI: 10.1155/2013/248512 (cit. on pp. 28, 29, 31-33, 36, 38, 40-43, 45, 46, 49, 74, 75, 97, 121, 180).

[17] M. C. Casabán et al. "Probabilistic solution of random homogeneous linear second-order difference equations". In: Appl. Math. Lett. 34.2 (2014), pp. 27-32. DOI: $10.1016 / \mathrm{j}$.aml.2014.03.0102 (cit. on pp. 4, 97).

[18] M. C. Casabán et al. "Probabilistic solution of random SI-type epidemiological models using the random variable transformation technique". In: Commun. Nonlinear. Sci. Numer. Simul. 24.1-3 (2015), pp. 86-97. DOI: 10.1016/j.cnsns. 2014.12.016 (cit. on pp. 54, 97, 137).

[19] M. C. Casabán et al. "Probabilistic solution of the homogeneous Riccati differential equation: A case-study by using linearization and transformation techniques". In: J. Comput. Appl. Math. 291 (2016), pp. 20-35. DOI: $10.1016 /$ j.cam. 2014.11 .028 (cit. on p. 75 ).

[20] G. Casella and R. L. Berger. Statistical Inference. New York: Brooks/Cole, 2002. ISBN: 9780534243128 (cit. on p. 4).

[21] C. Castillo-Chávez, W. Huang, and J. Li. "Competitive exclusion in gonorrhea models and other sexually-transmitted diseases". In: SIAM J. Appl. Math. 56.2 (1996), pp. 494-508. DOI: 10.1137/S003613999325419X (cit. on p. 54).

[22] W. M. Charles. Application of coloured noise as a driving force in the stochastic differential equations. Croatia: In Stochastic Control, 2010 (cit. on p. 2).

[23] W. M. Charles, E. van den Berg, and A. W. Heemink. "Coloured noise for dispersion of contaminants in shallow waters". In: Appl. Math. Model. 33.2 (2009), pp. 1158-1171. DOI: 10.1016/j .apm. 2008.01 .008 (cit. on p. 2).

[24] C. Chen and K. Yanmei. "Dynamics of a stochastic SIS epidemic model with saturated incidence". In: Abstract Appl. Anal. ID723825.3 (2014), pp. 1-13. DOI: $10.1155 / 2014 / 723825$ (cit. on p. 56 ). 
[25] D. Clancy. "A stochastic SIS infection model incorporating indirect transmission". In: J. Appl. Probab. 42.3 (2007), pp. 726-737. DOI: 10.1239/ jap/1127322023 (cit. on p. 56).

[26] CNMC, Comisión Nacional de los Mercados y la Competencia. http://data. cnmc.es/datagraph/jsp/inf_anual.jsp (cit. on pp. 123-125).

[27] R. Colucci and T. Caraballo. "A comparison between random and stochastic modeling for a SIR model". In: Commun. Pure Appl. Anal. 16 (2017), pp. 151-162. DOI: 10.3934/cpaa. 2017007 (cit. on p. 72).

[28] B. Cooper and M. Lipsitch. "The analysis of hospital infection data using hidden Markov models". In: Biostatistics 5 (2 2004), pp. 223-237. DOI: 10.1093/biostatistics/5.2.223 (cit. on p. 134).

[29] J. C. Cortés, L. Villafuerte, and L. Jódar. "Numerical solution of random differential initial value problems: Multistep methods". In: Math. Meth. Appl. Sci. 34.1 (2011), pp. 63-75. DOI: 10.1002/mma.1331 (cit. on p. 3).

[30] J. C. Cortés et al. "Applying the Wiener-Hermite Random technique to study the evolution of excess weight population in the region of Valencia (Spain)". In: AJCAM 2.4 (2012), pp. 274-281. DOI: 10.4236/ajcm. 2012. 24037 (cit. on p. 54).

[31] J. C. Cortés et al. "Random Airy type differential equations: Mean square exact and numerical solutions". In: Comput. Math. Appl. 60.5 (2010), pp. 1237-1244. DOI: 10.1016/j. camwa.2010.05.046 (cit. on p. 161).

[32] J. C. Cortés et al. "Solving Riccati time-dependent models with random quadratic coefficient". In: Appl. Math. Lett. 24.12 (2011), pp. 2193-2196. DOI: $10.1016 / \mathrm{j} . \mathrm{aml} .2011 .06 .024$ (cit. on p. 28).

[33] J. L. Doob. Stochastic Processes. New York: John Wiley and Sons Inc. Publications in Statistics, 1953. ISBN: 9780471523697 (cit. on p. 7).

[34] F. A. Dorini, M. S. Cecconello, and L. B. Dorini. "On the logistic equation subject to uncertainties in the environmental carrying capacity and initial population density". In: Commun. Nonlinear. Sci. Numer. Simul. 
33 (2016), pp. 160-173. DOI: $10.1016 /$ j . cnsns . 2014.12.016 (cit. on p. 137).

[35] F. A. Dorini, Bobko N., and L. B. Dorini. "A note on the logistic equation subject to uncertainties in parameters". In: Comp. Appl. Math. (2016), pp. 1-11. DOI: 10.1007/s40314-016-0409-6 (cit. on p. 137).

[36] M. El-Beltagy and M. El-Tawil. "Toward a solution of a class of nonlinear stochastic perturbed PDEs using automated WHEP algorith". In: Appl. Math. Model. 37.12-13 (2013), pp. 7174-7192. DOI: 10 .1016/j . apm.2013.01.038 (cit. on p. 2).

[37] M. A. El-Tawil. "The approximate solutions of some stochastic differential equations using transformations". In: Appl. Math. Comput. 164.1 (2005), pp. 167-178. DOI: 10.1016/j.amc.2004.04.062 (cit. on p. 4).

[38] C. Fernández, F. J. Vázquez, and J. M. Vegas. Differential and Difference Equations. Dynamical Systems. Madrid: Thomson, 2003 (in Spanish). ISBN: 9781461473336 (cit. on p. 161).

[39] A. Friedman. Stochastic Differential Equations and Applications. New York: Academic Press, 1975. ISBN: 9783540047582 (cit. on p. 4).

[40] T. C. Gard. Introduction to Stochastic Differential Equations. New York: Marcel Dekker, 1988. ISBN: 9781470410544 (cit. on pp. 2, 180).

[41] R. G. Ghanem and P. D. Spanos. Stochastic Finite Elements: A Spectral Approach. New York: Courier Dover Publ., 2003. ISBN: 0486428184 (cit. on p. 3).

[42] I. I. Gikhman and A. V. Skorokhod. Stochastic differential equations. New York: Springer-Verlag, 1975. ISBN: 9783540047582 (cit. on p. 4).

[43] A. K. Golmankhaneh, N. A. Porghoveh, and D. Baleanu. "Mean square solutions of second-order random differential equations by using homotopy analysis method". In: Rom. Rep. Phys. 65 (2 2013), pp. 350-362 (cit. on p. 161). 
[44] G.R. Grimmett and D. Stirzaker. Probability and Random Processes. Oxford: Claredon Press, 2000. ISBN: 9781493300266 (cit. on p. 12).

[45] G. Hazen and Z. Li. "Cohort decomposition for Markov cost-effectiveness models". In: Med. Decis. Making. 31.1 (2011), pp. 19-34. DOI: 10.1177/ 0272989X10368999 (cit. on p. 146).

[46] H. W. Hethcote. "Biomathematics". In: vol. 18. Applied Mathematical Ecology. Heidelberg: Springer-Verlag, 1989. Chap. Three Basic Epidemiological Models, pp. 120-144 (cit. on pp. 54, 55).

[47] H. W. Hethcote. "The mathematics of infectious diseases". In: SIAM Review 42.4 (2000), pp. 599-653. DOI: 10.1137/S0036144500371907 (cit. on p. 54).

[48] H. W. Hethcote and J. A. Yorke. Gonorrhea Transmission Dynamics and Control. Vol. 56. Lecture Notes in Biomathematics. Berlin: SpringerVerlag, 1984 (cit. on p. 54).

[49] A. Hussein and M. M. Selim. "A developed solution of the stochastic Milne problem using probabilistic transformations". In: Appl. Math. Comput. 216.10 (2009), pp. 2910-2919. DOI: 10.1016/j . amc. 2010.04. 003 (cit. on p. 4).

[50] A. Hussein and M. M. Selim. "Solution of the stochastic radiative transfer equation with Rayleigh scattering using RVT technique". In: Appl. Math. Comput. 218.13 (2012), pp. 7193-7203. DOI: 10.1016/j . amc.2011.12. 088 (cit. on p. 4).

[51] A. Hussein and M. M. Selim. "Solution of the stochastic transport equation of neutral particles with anisotropic scattering using RVT technique". In: Appl. Math. Comput. 213.1 (2009), pp. 250-261. DOI: 10.1016/j . amc.2009.03.016 (cit. on p. 4).

[52] S. Kadry. "On the generalization of probabilistic transformation method". In: Appl. Math. Comput. 190.15 (2007), pp. 1284-1289. DOI: 10.1016/ j.amc.2007.02.009 (cit. on p. 4). 
[53] G. Karlsson and M. Johannesson. "The decision rules of cost-effectiveness analysis". In: PharmacoEconomics 9.2 (1996), pp. 113-120. DOI: 10 . 2165/00019053-199609020-00003 (cit. on p. 145).

[54] B. Kegan and R. W. West. "Modeling the simple epidemic with deterministic differential equations and random initial conditions". In: Math Biosci 195.5 (2005), pp. 179-193. DOI: $10.1016 /$ j .mbs . 2005.02 .004 (cit. on pp. 54, 137).

[55] A. R. Khudair, S. A. M. Haddad, and S.L . Khalaf. "Mean square solutions of second-order random differential equations by using the differential transformation method". In: Open J. Appl. Sci. 6 (2016), pp. 287297. DOI: 10.4236/ojapps.2016.64028 (cit. on p. 161).

[56] S. Kindermann and S̆. Papáček. "On Data Space Selection and Data Processing for Parameter Identification in a Reaction-Diffusion Model Based on FRAP Experiments". In: Abstract Appl. Anal. ID 859849 (2015), pp. 1-17 (cit. on p. 84).

[57] P. E. Kloeden and E. Platen. Numerical Solution of Stochastic Differential Equations. 3th. Vol. 23. Applications of Mathematics: Stochastic Modelling and Applied Probability. New York: Springer, 1999. ISBN: 9783540540625 (cit. on p. 2).

[58] I. A. Korostil et al. "Adaptive Markov chain Monte Carlo forward projection for statistical analysis in epidemic modelling of human papillomavirus". In: Stat. Med. 32.11 (2012), pp. 1917-1953. DOI: 10.1002/sim. 5590 (cit. on p. 134).

[59] D. P. Kroese, T. Taimre, and Z. I. Botev. Handbok of Monte Carlo Methods. New Jersey: Wiley, 2011. ISBN: 9781118014967 (cit. on p. 3).

[60] M. Loève. Probability Theory I and II. N. Jersey: Van Nostrand-Reinhold, 1963. ISBN: 9780387902104-9780387902623 (cit. on p. 7).

[61] G. J. Lord, C. E. Powell, and T. Shardlow. An Introduction to Computational Stochastic PDEs. Cambridge Texts in Applied Mathematics. New York: Cambridge University Press, 2014. ISBN: 9780521728522 (cit. on pp. 183, 207, 210). 
[62] M. Mandel and R. A. Betensky. "Estimating time-to-event from longitudinal ordinal data using random-effects Markov models: application to multiple sclerosis progression". In: Biostatistics 9 (4 2008), pp. 750-764 (cit. on p. 134).

[63] J. Mar, M. Sainz-Ezkerra, and E. Miranda-Serrano. "Calculation of prevalence with Markov models: Budget impact analysis of thrombolysis for stroke". In: Med. Decis. Making. 28.4 (2008), pp. 481-490. DOI: 10.1177/ 0272989X07312720 (cit. on p. 134).

[64] J. Mar et al. "Los modelos de Markov probabilísticos en la evaluación económica de tecnologías sanitarias: una guía práctica". In: Gac. Sanit. 24.3 (2010), pp. 209-214. DOI: 10.1016/j.gaceta.2010.02.006 (cit. on pp. 112, 134-136, 146-148, 154).

[65] Mathematica. http://www.wolfram.com/mathematica (cit. on pp. 68, 78).

[66] L. A. McNutt et al. "Estimating the Relative Risk in Cohort Studies and Clinical Trials of Common Outcomes". In: Am. J. Epidemiol. 157.10 (2003), pp. 940-943. DOI: 10.1093/aje/kwg074 (cit. on p. 136).

[67] M. Morales and L. W. González. "Edad y crecimiento del pez Haemulon Steindachneri Perciformis: Haemulidae en el suroeste de la isla de Margarita Venezuela". In: Rev. Biol. Trop 58.1 (2010), pp. 299-310. DOI: 10.15517/rbt.v58i1.5211 (cit. on p. 82).

[68] National Spanish Health Survey (Encuesta Nacional de Salud de España, ENSE). http://pestadistico.inteligenciadegestion.msssi.es/ (cit. on p. 66).

[69] J. A. Nelder and R. Mead. "A simplex method for function minimization". In: Computer Journal 7.1 (1964), pp. 308-313. DOI: 10.1093/comjnl/7. 4.308 (cit. on p. 68).

[70] R. B. Nelsen. An Introduction to Copulas. New York: Springer, 1999. ISBN: 9780387286594 (cit. on p. 2).

[71] B. Øksendal. Stochastic Differential Equations: An Introduction with Applications. 6th. Springer, 2010. ISBN: 9783540047582 (cit. on p. 2). 
[72] A. Papoulis. Probability, Random Variables and Stochastic Processes. New York: McGraw Hill, 1991. ISBN: 9780070484771 (cit. on p. 7).

[73] J. L. Pinto-Prades, J. Puig-Junoy, and V. Ortún-Rubio. "Análisis costeutilidad". In: Aten. Primaria 27 (2001), pp. 569-573. DOI: 10.1016/ S0212-6567(01)78861-0 (cit. on p. 146).

[74] L. Prieto et al. "Análisis coste-efectividad en la evaluación económica de intervenciones sanitarias". In: Med. Clin. 122.13 (2004), pp. 505-510. DOI: $10.1157 / 13060174$ (cit. on p. 145).

[75] L. v. Qiming and J. W. Pitchford. "Stochastic von Bertalanffy models with applications to fish recruitment". In: J. Theor. Biol 244 (2007), pp. 640-655. DOI: 10.1016/j.jtbi.2006.09.009 (cit. on p. 82).

[76] W. T. Reid. Riccati Differential Equations. New York: Academic Press, 1972. ISBN: 9780125862509 (cit. on p. 28).

[77] E. M. Rogers. Diffusion of Innovations. New York: Free Press, 2003. ISBN: 9780743222099 (cit. on p. 54).

[78] J. A. Sacristán et al. "¿Qué es una tecnología sanitaria eficiente en España?" In: Gac. Sanit. 4.16 (2002), pp. 334-343. DOI: 10.1016/S02139111(02)71933-X (cit. on pp. 154, 156).

[79] Gema Calbo Sanjuán. "Mean Square Analytic Solutions of Random Linear Models". PhD thesis. Departamento de Matemática Aplicada. Universitat Politècnica de València, 2010 (cit. on p. 7).

[80] B. Sericola. Markov Chains: Theory, Algorithms and Applications. Applied stochastic methods series. ISTE Ltd, 2013. ISBN: 9781848214934 (cit. on pp. 111, 112, 120, 122).

[81] M. A. Shah. "Stochastic logistic model for fish growth". In: OJS. 4 (2014), pp. 11-18. DOI: 10.4236/ojs. 2014.41002 (cit. on p. 82).

[82] S. S. Shapiro and M. B. Wilk. "An analysis of variance test for normality". In: Biometrika 4.3-4 (1965), pp. 591-611. DOI: 10.1093/biomet/52.34.591 (cit. on p. 85). 
[83] R. C. Smith. Uncertainty Quantification: Theory, Implementation, and Applications. Philadelphia: SIAM, 2014. ISBN: 9781611973211 (cit. on pp. 84, 87).

[84] F. A. Sonnenberg and R. Beck. "Markov models in medical decision making: a practical guide". In: Med. Decis. Making 13.4 (1993), pp. 322-338. DOI: $10.1177 / 0272989 \times 9301300409$ (cit. on pp. 134, 146).

[85] T. T. Soong. Random Differential Equations in Science and Engineering. New York: Academic Press, 1973. ISBN: 9780126548501 (cit. on pp. 2, 4, $7,11,16,19,21,57,58,161,180,185)$.

[86] J. L. Strand. "Random ordinary differential equations". In: Journal of Differential Equations 7 (1970), pp. 538-553. DOI: 10.1016/0022-0396(70) 90100-2 (cit. on p. 180).

[87] A. Tarantola. Inverse Problem Theory and Methods for Model Parameter Estimation. Philadelphia: SIAM, 2005. ISBN: 9780898715729 (cit. on p. 84).

[88] G. Teschl. Ordinary Differential Equations and Dynamical Systems. Providence: American Mathematical Society, 2012. ISBN: 9780821883280 (cit. on pp. 161, 164).

[89] H. Tong. Nonlinear Time Series: A Dynamical System Approach. Oxford: Clarendon Press, 1990. ISBN: 9780198523000 (cit. on p. 2).

[90] F. Verhulst. Nonlinear Differential Equations and Dynamical Systems. Berlin Heidelberg: Springer-Verlag, 1996. ISBN: 9783540609346 (cit. on p. 161).

[91] L. Villafuerte and B. M. Chen-Charpentier. "A random differential transform method: Theory and applications". In: Appl. Math. Lett. 25 (10 2012), pp. 1490-1494. DOI: $10.1016 / \mathrm{j}$. aml . 2011.12.033 (cit. on p. 161).

[92] L. Villafuerte et al. "Random differential operational calculus: Theory and applications". In: Comput. Math. Appl. 59.1 (2010), pp. 115-125. DOI: $10.1016 /$ j.camwa.2009.08.061 (cit. on p. 161). 
[93] S. Wang, S. Song, and Y. Wang. "Skew Ornstein Uhlenbeck processes and their financial applications". In: J. Comput. Appl. Math. 273.1 (2015), pp. 363-382. DOI: 10.1016/j.cam.2014.06.023 (cit. on p. 2).

[94] Wildernessnorth. http://wildernessnorth.com/wordpress/wp-content/ uploads/2014/09/ (cit. on p. 84).

[95] E. Wong and B. Hajek. Stochastic Processes in Engineering System. New York: Springer Verlag, 1985. ISBN: 9781461295457 (cit. on p. 7).

[96] D. Zhang and A. Ntoko. "Computational Methods in Decision-Making, Economics and Finance". In: vol. 74. Applied Optimization. New York: Springer, 2002. Chap. Mathematical Model of Technology Diffusion in Developing Countries, pp. 526-539 (cit. on p. 54).

[97] J. Zhang and K. F. Yu. "What's the relative risk? A method of correcting the odds ratio in cohort studies of common outcomes". In: JAMA 290.19 (1998), pp. 1690-1691 (cit. on p. 136). 
